\title{
UMA EXTENSÃO DA TECNICA AID \\ EM MODELOS LINEARES GENERALIZADOS
}

\author{
MARI A CECILIA MENDES BARRETO \\ Estatistico
}

Orientadora: Profa. Dra. CLARICE GARCIA BORGES DEMÉTRIO

Tese apresentada à Escola Superior de Agricultura "Luiz de Queiroz", da Universidade de São Paulo, para obtenção do título de Doutor em Agronomia, Área de Concentração: Estatística e Experimentação Agronómica.

PIRACICABA

Estado de Săo Paulo - Brasil

maio de 1993 
Barreto, Maria Cecilia Mendes

Be73e .... Uma extensão da técnica AID em modelos I ineares generalizados. Piracicaba, 1993.

200 .

\section{Tese - ESALQ}

Bibliografia.

1. Estatistica matematica 2 . Modelo matemático 3. Técnica AID I. Escola Superior de Agricultura Luiz de Queiroz, Piracicaba.

CDD 518.8 519.5 
UMA EXTENSÃO DA TECNICA AID

EM MODELOS LINEARES GENERALIZADOS

MARIA CECILIA MENDES BARRETO

Aprovada em: 18.08 .1993

Comissão julgadora:

Profa. Dra. Clarice Garcia Borges Demétrio ESALQ/USP Prof. Dr. Carlos Roberto Padovani IB/UNESP

Prof. Dr. Euclides Braga Mal heiros ... FGA UNESP

Prof. Dr. Decio Barbin ESALQNUSP

Prof. Dr. Hilton Thadeu Zarete do Couto ESALQUSP

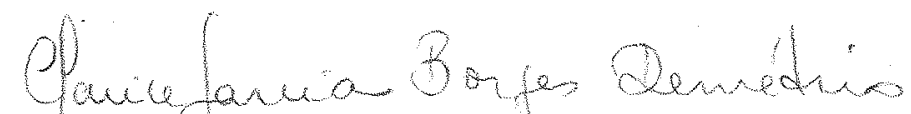

Profa. Dna. CLARICE GARCIA BORGES DEMETRIO Orientadora 
Aos queridos

Flávio Cesar.

Guilherme, Heitor,

Hermínio e Apparecida. 
Agradeço:

- A Profa. Dra. Clarice Garcia Borges Demetrio pela orientação, dedicação e estímulo;

- e a todos aqueles que de alguma forma colaboraram para que este trabalho pudesse ser concluido. 


\section{SUMARIO}

Página

RESUMO

vii

SUMMARY

ix

1. INTRODUÇAO

1.1. Motivaçăo ................... 1

1.2. Objetivos ................ 2

1.3. A organização deste trabalho ........... 3

2. ALGUNS ALGORITMOS DE DIVISAO DI COTOMICA HIERARQUTCA $\ldots \ldots \ldots \ldots \ldots \ldots \ldots \ldots \ldots \ldots$

2.1. Introdução $\ldots \ldots \ldots \ldots \ldots \ldots \ldots \ldots \ldots \ldots \ldots$

2. 2. Partiçăo dicotômica $\ldots \ldots \ldots \ldots \ldots \ldots \ldots \ldots$

2.3. Criterios de partiçăo e regras de parada. 11

2.4. O método de agr upamento divisivo hierárquico

2.5. Critérios para a escolha do próximo grupo

2.5.1. Agrupamento para homogeneidade máxima................. ze

2. 5. 2. Técnica AID ............... 23

2. 5. 3. Agrupamento de casos e variáveis ... 24

2.5. 4. Teste de máxima verossimilhança ... 26

3. UMA INTRODUÇO AOS MODELOS LINEARES GENERALIZADOS

3.1. O ajuste de un modelo $\ldots \ldots \ldots \ldots \ldots \ldots \ldots$

3. 2. Os componentes de um modelo linear generalizado ....................

3.3. Inferencias sobre o modelo linear 
3.4. o algortimo de estimaçăo ...............

4. UMA MEDIDA MAIS GERAL DE HOMOGENEIDADE DE GRUPOS

E ALGUMAS CONSEQUENCIAS ............... 45

4.1. Introduçăo ..................... 45

4.2. Uma extensão natural para a medida de homogeneidade de grupos ..............

4.3. o caso normal .................... 50

4.4. O caso Poisson .................... 5e

4.5. o caso binomial ................ 54

4.6. A escolha da divisão mais promissora ..... 63

5. UMA DEFINIGAO MAIS GERAL DAS ESTATISTICAS E

UMA ADAPTAÇZO AO ALGORITMO DE AGRUPAMENTO

DIVISIVO HIERARQUTCO .................. 70

5.1. Introdução ...................... 70

5. 2. A estatistica $R^{2}$ generalizada ......... 70

5.2.1. o caso normal .............. 74

5.2.2. o caso Poisson ............... 76

5.2.3. o caso binomial ............ 78

5. 3. O algoritmo de agrupamento hierárquico divisivo ...................... 83

5. 4. Uma generalização do teste de SCOTT \& KNOTT $(1974) \ldots \ldots \ldots \ldots$

6. ALGUNS RESULTADOS SOBRE A DISTRIBUIGAO DO MAXIMO DA ESTATISTICA $\triangle C \hat{\mu}_{d}$, NO CASO NORMAL .......... 89

6.1. Introduça $\ldots \ldots \ldots \ldots \ldots \ldots . \ldots \ldots$

6. 2. O critério $B$ sob a regra de divisão monotônica e mesmo número de observaços por casela...................... 89

6.2.1. Uma transformaçăo conveniente ..... 90

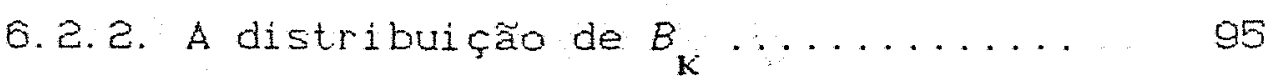

6.2.3. Aproximação por uma distribuição

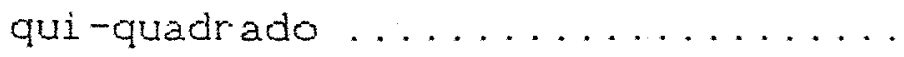


6.3. O criterio $B$ sob a regra de divisão monotonica e número diferente de observaçes por casela ...................... 98

6.3.1. A distribuição de $B_{K} \ldots . . . . . . .100$

6. 3.2. Limites inferiores para a distribuiçăo de $B_{K}$ usando desigualdades ....... 102 6. 3.3. Limites para a distribuifăo de $B_{K}$ usando a distribuição qui-quadrado

6. 3. 4. Al gumas concl usöes ..............

6.4. O criterio $B$ sob a regra de divisão livre e mesmo número de observaçóes por casela ...

6.4.1. A distribuição de $B_{k} \ldots \ldots \ldots \ldots$

6.4.2. Desigualdades que 1 imitam a distribuição de $B_{K} \ldots \ldots \ldots \ldots \ldots$

6.4.3. A distribuif̧̃̃o de qui-quadrado como uma aproximação da distribuiçăo de $B_{K}$

6.5. O critério $B$ sob a regra de divisăo 1 ivre e número diferente de observaçóes por casela 6.5.1. A função distribuição acumulada de $B_{K}$

6. 5. อ. Distribuiçôes que limitam a distribuiço de $B_{K} \ldots \ldots \ldots \ldots \ldots$

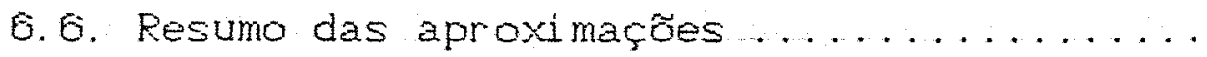

7. UM ESTUDO SOBRE O COMPORTAMENTO DA DISTRIBUI GAO DO MAXIMO DA ESTATISTICA $\triangle C \hat{\sim} \mathrm{d}$, NO CASO BINOMIAL

7.1. Introduça

7.2. Descrição do programa de simulação...... 121

7.3. A distribuifão simulada de $\Delta(\hat{\pi}) \ldots$....... $12 \mathrm{e}$

7.3.1. O caso de 10 tratamentos ......... 124

8.3.2. O caso de 6 tratamentos .......... 120 
8. 3. 3. o caso de 3 tratamentos ........ 127

7.4. Conclusoes ...................... 129

8. o algoritmo AID .................... 136

8.1. Descrição do algoritmo AID ........... 136

8. 2. Algumas al ternativas sobre criterios de seleção e regras de parada ............. 140

8.3. Um procedimento para valores pequenos de $B$

8. 4. Procedimentos para valores grandes do criterio $B \ldots \ldots \ldots \ldots \ldots \ldots . \ldots \ldots$

8.5. Uma boa aproxi maçăo para a distribuiçăo de $B_{K}$

8.6. Uma modificação do algoritmo AID para dados da familia exponencial ..............

9. A UTILIZAGAO DO ALGORITMO AID EM DADOS BINOMIAIS

9.1. Uma análise de um experimento com fungicidas

9. 2. Resultados da aplicação do algoritmo AID. 154 10. CONCLUSOES ........................... 159 REFERENCIAS EIBLIOGRAFICAS ............. 161

APENDICE 1: O PROGRAMA DE SIMULAGAO .......... 166 AFENDICE 2: RESULTADO DE UMA DAS SIMULACOES ...... 171 APENDTCE 3: LISTAGENS DO PROGPAMA E DA EXECUCAO DO PROGRAMA DE ANALISE DE AGRUPAMENTOS EM LINGUAGEM GLIM ................... 182 
UMA EXTENSEO DA TECNICA AID

EM MODELOS LINEAFES GENERALIZADOS

Autor a: MARIA CECILIA MENDES BARRETO

Orientadora: PROFA. DRA. CLARICE GARCIA BORGES DEMETRIO

\section{RESUMO}

Como as técnicas de agrupamentos de médias podem ser inadequadas na aplicação em dados com distribuição diferente da gaussiana, o algoritmo 'Automatic Interaction Detection' (AID) estendido adotando-se como medida de homogeneidade de grupos uma estatistica baseada na função desvio.

Esta última pode ser utilizada em uma grande classe de modelos, conhecida como modelos lineares generalizados, que abrange modelos do tipo regressão, análise de variancia, modelos logito e probito, modelos log-lineares, entre outros.

Considerando um ensaio completamente ao acaso com $k$ tratamentos e $n_{k}$ repetiçães por tratamento, o máximo da medida de homogeneidade de grupos e uma extensão do coeficiente de determinaçăo săo obtidos em uma forma geral supondo que os dados tenham uma distribuiçăo que pertença à familia exponencial, e também no caso particular de distribuiçăo normal, binomial $e$ Poisson.

Supondo que os dados tenham distribuição binomial, a distribuição assintótica do máximo da medida 
de homogeneidade de grupos é obtida como sendo proporcional a uma distribuição qui-quadrado.

Esses resultados são, tambem, uma extensão do procedimento de SCOTT \& KNOTT (1974), para agrupamento de médias. 
AN EXTENSION OF THE AID TECHNIQUE

IN GENERALIZED LINEAR MODELS

Author: MARIA CECILIA MENDES BARRETO Adviser: PROFA. DRA. CLARICE GARCIA BORGES DEMETRIO

\section{SUMMARY}

Since Clustering techniques for means can be inadequate for proportions with non-Gaussian data, the AID C Automatic. Interaction Detection 2 algorithm is extended considering as measure of homogeneity of groups a statistics based on the deviance function.

This can be used in a large class of models known as generalized linear models. These models have, as particular case, among others, standard regression and analysis of variance models, logit and probit models, log-linear models for contingency tables, etc.

Considering a complety random design with $K$ treatments and $n_{k}$ replicates per treatment, the maximum of the groups homogeneity meeasure is obtained. Also, it is obtained an extension of the "determination coefficient. "In a general form, supposing a distribution for the data in the exponential family; in particular the normal, binomial and Poisson cases are considered.

Supposing a binomial distribution for the data, the asymptotic distribution for the maximum of the group homogeneity measure is obtained, it is shown that 
it is proportional to a chi-square distribution. These results are also, an extension of the procedures proposed by SCOTT \& KNOTT (1974) for clustering means. 
1. INTRODUÇÃO.

1.1. Moti vação

Uma das situaçōes mais frequentes encontradas por pesquisadores de diversas áreas do conhecimento é formar grupos de individuos que sejam, de alguma maneira, similares.

A análise de agrupamentos é um conjunto de técnicas e algoritmos cujo objetivo é encontrar e separar individuos em grupos similares a partir do conhecimento de $\rho$ variáveis que os definem.

Entretanto, algumas vezes, alem de se ter um conjunto de $\rho$ variáveis preditoras, tem-se também uma variavel resposta definida para cada individuo. Um dos procedimentos existentes para a busca de grupos similares nessa situaçăo, é a técnica AID ("Automatic Interaction Detection"' que foi inicialmente proposta por MORGAN \& SONQUIST (1963).

O metodo de agrupamento de médias de SCOTT \& KNOTT (1974) é um caso particular da técnica AID, e usa - metodo de análise de agrupamento divisivo hierárquico dicotémico para separar as médias amostrais de tratamentos em grupos razoavel mente homogeneos no caso de del ineamentos bal anceados; quando o resul tado de um teste 
F na análise de variancia é significativo.

COX \& SPJOTVOLL (1982) usam como procedimento o teste das hipóteses de que os dados estão, primeiramente, em dois grupos homogêneos, e caso não haja nenhuma partição satisfatoria, em um segundo passo săo testadas todas as partiçoses em três grupos e assim por diante. A estatistica utilizada é baseada no teste $F$ tradicional.

CARMER \& LIN (1983) fazem um estudo do erro do tipo 1 para métodos de agrupamento divisivo de médias de grupos, que formam conjuntos disjuntos de tratamentos.

Considerando um ensaio inteiramente ao acaso do comportamento de fungicidas sobre a porcentagem de germinação de sementes de milho, não é adequado se utilizarem esses métodos na análise de variancia usual para a transformaça arco seno da raiz quadrada da proporção de sementes germinadas, uma vez que o interesse do pesquisador está em um agrupamento na variavel original.

Nessa situação é indicada a análise de modelo probito ou logito, seguida de uma técnica de agrupamento que se adapte à variável resposta binaria.

1.2. Objetivos.

A medida de homogeneidade entre grupos usada, por exemplo, por SCOTT \& KNOTT (1974), corresponde à soma de quadrados entre dois grupos, em un delineamento completamente ao acaso. Um dos primeiros autores a 
definir homogeneidade entre grupos foi FISHER (1958) propondo a soma de quadrados ponderada.

Entretanto, a partir do desenvolvimento de modelos lineares generalizados (MGL) por NELDER \& WEDDERBURN (1972) um grande número de métodos estatisticos, tais como modelos de regressão, modelos probito e logito, modelo de médias, modelos log-lineares, modelos de regressão de Cox, dentre outros, pode ser escrito de uma maneira unificada, possibilitando, também, que a função desvio seja usada como medida de qualidade de ajuste do modelo em questão.

Esses conceitos permitem estender 0 algoritmo AID para uma classe mais ampla de situaçóes onde se faz necessária a aplicação de métodos divisivos hierárquicos.

1.3. A organização deste trabalho.

Com o objetivo de se propor uma modificação na técnica AID para que se possa utilizar um método de divisão dicotónica hierárquica em análise de agrupamentos para um grande número de modelos estatisticos, este trabalho estuda, em particular, o caso de resposta binária, onde os modelos lagito e probito são casos típicos, bem como o caso de resposta categorizada, onde os modelos log-lineares săo utilizados para a análise.

O Capitulo e trata de algoritmos de divisăo dicotomica hierarquica, onde se faz referência ao procedimento de SCOTT \& KNOTT $(1974)$ e ao algoritmo AID. 
Uma breve introdução aos modelos lineares generalizados, que é apresentada no Capítulo 3 , permite estender o conceito de homogeneidade entre grupos para dados da familia exponencial (Capitulo 4). Desse modo. uma definição mais geral das estatisticas usadas no algoritmo AID são estendidas para a familia exponencial no Capítulo 5.

Um resumo dos resultados a respeito da distribuição do máximo da medida de homogeneidade entre grupos no caso de distribuiçăo normal, está no Capítulo 6 e no caso de distribuiça bi nomial, no Capitulo 7 .

Uma descriçăo mais detalhada do algoritmo AID e algumas modificações em seus passos estão no Capítulo 8.

Una das possiveis versóes do algoritmo AID, usando a medida de homogeneidade entre grupos no caso binomial, é discutida em um modelo logito no Capitulo 9.

- Capitulo 10 apresenta as conclusós deste trabalho.

Una cópia do programa utilizado para se obterem os resultados da simulação está no Apêndice 1. uma das situaçóes estudadas, no Apendice $e$. O programa para aplicação do algoritmo AID estendido está no Apendice 3 . 


\section{ALGUNS ALGORI TMOS DE DIVISÃO DICOTOMICA HIERARQUICA}

\subsection{Introdução}

$\mathrm{Na}$ comparação de médias de tratamentos, por exemplo em análise de variancia, algumas vezes é de interesse apresentá-las agrupadas, de tal modo que os tratamentos do mesmo grupo possam ser considerados como tendo a mesma média.

Quando o número de niveis do fator ou o número de variáveis preditoras é muito grande, a escol ha de um modelo adequado e sua subsequiente avaliação podem se tornar trabalhosas e na maioria dos casos de dificil interpretação. Técnicas alternativas baseadas em análise de agrupamentos podem ser usadas, pois, alem de serem conceitualmente mais intuitivas, em geral, produzem resultados mais simples de se interpretarem.

o método AID C"Automatic Interaction Detection") proposto por MORGAN \& SONQUIST (1963) como uma das técnicas de analise de agrupamentos, consiste em dividir o conjunto de dados sucessivamente em grupos, combinando ou não as classes das variaveis preditoras ou niveis dos fatores, de modo a produzir grupos que se diferenciem ao máximo em relação à média da variável resposta. O processo de subdivisão e aplicado enquanto as 
di visões obtidas contribuem para explicar a variaça dos dados e ao final tem-se um conjunto de grupos que podem ser identificados por alguns niveis dos fatores ou variáveis preditoras e suas interaçőes mais importantes.

2. 2. Partiçăo dicotômica

Para se dividir um conjunto de dados em grupos homogeneos, uma soluçăo seria examinar todas as partiçoses possiveis do referido conjunto e escolher aquela que é ótima segundo algum critério. Entretanto. conforme observou FISHER (1958), por conhecimento anterior, teoria ou conveniência, algumas partiçóes são eliminadas como sendo soluçōes possiveis. Um caso típico é quando as observaçöes são tomadas sucessivamente durante alguns anos, e deseja-se agrupar os dados em ordem cronológica. Esse fato em si elimina um grande número de partiçóes possiveis, desde que se busquem apenas grupos formados por elementos consecutivos.

Como em cada problema tem-se um conjunto de regras que determinam as partiços possiveis, diz-se que uma regra $\gamma$ pode ser classificada como livre se nenhuma condiçăo é imposta às partiçoes, ou então como restrita, caso certas condiços sejam levadas em consideração. Dentre as restritas, se os grupos formados tiverem elementos consecuti vos com relaçăo a al guma or dem fixada no conjunto $(1,2, \ldots, k)$ de indices das observaçoes, a regra é monotónica. Se, por outro lado, os grupos são formados por outro tipo de restriça, entăo a regra é complexa. Indica-se $\gamma_{\mathrm{L}}$ uma regra livre e $\gamma_{\mathrm{M}}$ uma 
regra monotónica. Mostra-se que quando da aplicaçăo de regra $\gamma_{L}$, tem-se $e^{K-1}-1$ partiçoes possiveis, e quando da regra $\gamma_{M}, K-1$ partições possiveis.

No exemplo onde os grupos são formados em ordem cronológica tem-se uma regra $\gamma$ monotônica. o algoritmo de HARTIGAN (1972), que apresenta um modelo e uma técnica para agrupar simultaneamente casos e variaveis, é um exemplo de regra complexa. O trabalho de BUSSAB (1976) apresenta em detalhes todo o desenvolvimento de regras de agrupamentos, em particular da regra $\gamma$.

Sem perda da generalidade, considere-se o conjunto de observações dispostas em $\mathrm{K}$ caselas, que serão agrupadas e o conjunto de indices

$$
I=\{1,2, \ldots, k\}
$$

de tal maneira que quando se fizer uma partição das caselas, escrever-se-a uma partiçăo do conjunto $I$.

Ao se aplicar uma regra $\gamma$ a um conjunto de indices, I, tem-se uma partiçăo dicotomica, se esta última é constituida de dois grupos. Seja W( $I, \gamma)$ o conjunto de todas as partiçóes dicotomicas possiveis $\gamma$ do conjunto 1 . Qualquer elemento $d$ de $\Psi(I, y)$ e formado por dois subconjuntos $\gamma(\alpha)$ e $L(d)$, que são complementares em I, ou seja

$$
J(\alpha) \cap L(\alpha)=0 \text { e } J(\alpha) \cup L(\alpha)=I
$$

$$
\text { For exemplo, para } I=\{1,2,3,4,5,0) \text {, }
$$

tem-se que: 
- Aplicando a regra livre $\gamma_{L}$, o conjunto $\Psi I, \gamma_{L}{ }^{2}$ contém 31 elementos.

- Considerando a regra monotónica $\gamma_{M}$, onde a ordem fixada é a natural, $\left.\Psi I, \gamma_{M}\right)$ contém 5 elementos, $\alpha_{i}$, que estão na Tabela 1. Convém notar que se a ordenação não fosse a natural, os elementos do conjunto $\Psi\left(I, \gamma_{M}{ }^{3}\right.$ seriam completamente diferentes daqueles encontrados.

Tabela 1 - Elementos do conjunto $\Psi I \cdot \gamma_{M}{ }^{2}$ onde $I=\langle 1,2,3,4,5,6\}$ e a ordem monotônica é a nat.ural.

\begin{tabular}{ccc}
\hline$d$ & $j(d)$ & $L(d)$ \\
\hline$d_{1}$ & $(1\}$ & $\{2,3,4,5,6)$ \\
$d_{2}$ & $(1,2)$ & $(3,4,5,6)$ \\
$d_{3}$ & $(1,2,3)$ & $(4,5,6)$ \\
$d_{4}$ & $(1,2,3,4\}$ & $\{5,6)$ \\
$d_{5}$ & $\{1,2,3,4,5\}$ & $(0)$ \\
\hline
\end{tabular}

- Se o conjunto I corresponde a caselas em uma classificação dupla, sendo um fator com 2 niveis e o outro com 3, ele tera 6 elementos que podem ser representados através de 


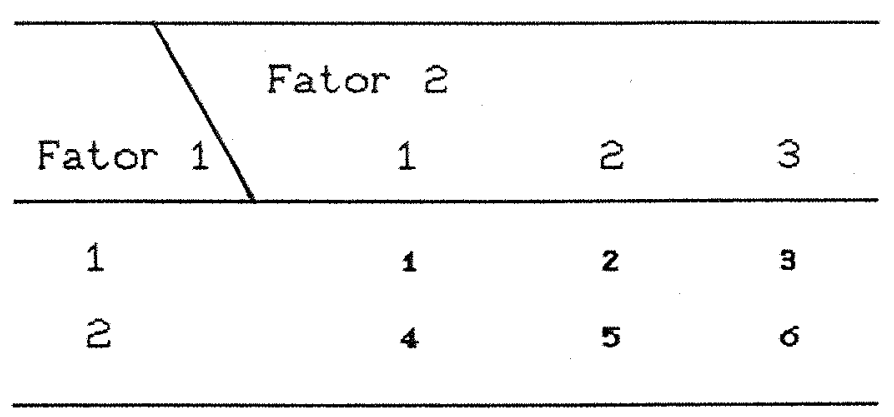

Então o conjunto de 1 ndices das caselas tem a seguinte correspondencia com o conjunto de indices $I$ :

$$
\{(1,1),(1,2),(1,3),(2,1),(2,2),(2,3)\}=\{1,2,3,4,5,6\}
$$

Considerando que a regra $\gamma$ permite partições baseadas em agr upamentos marginais apenas com partiçöes monotónicas para o fator 2 , tem-se que $\Psi(1, \gamma)$ contém 3 elementos, $d_{3}, d_{0}, d_{7}$, que estão na tabela 2 .

Tabela $Z$-Elementos do conjunto $\Psi(I, \gamma)$, onde $I=\{1,2,3,4,5,0\}$ e a regra $\gamma$ permite agrupamentos marginais com partiçôes monotonicas para o Fator 2 .

$\begin{array}{lcc}d & j(d) & l(\alpha) \\ d_{3} & (1,2,3) & \{4,5,0) \\ d_{0} & (1,4) & \{2,3,5,0\} \\ d_{7} & \{1,2,4,5\} & \{3,0\}\end{array}$


Se $d$ é um elemento de $\Psi(1, \gamma)$, então $d$ é formado por dois subconjuntos de $I, \mathcal{K}(\alpha)$ e $L(\alpha)$. Por outro lado, se for considerada a classe $\Psi(L(d), \gamma)$, cada um de seus elementos $d$ ' formara novamente dois subconjuntos de $L(d), \mathcal{K}\left(d^{\prime}\right)$ e $L\left(d^{\prime}\right)$. Assim o conjunto $\left\{J(d), \mathcal{J}\left(d^{\prime}\right), L\left(d^{\prime}\right)\right\}$ é formado por uma tripartição do conjunto 1 , obtida por duas divisões dicotómicas $\gamma$ sucessivas. Define-se então a tripartição de I formada deste modo como o resultado de um produto especial

$J(d) *(L(\alpha), \gamma)=\left(\left[J(d), J\left(\alpha^{\prime}\right), L\left(d^{\prime}\right)\right]: d^{\prime} \in \Psi(L(\alpha), \gamma)\right)$

Pode-se, então, construir a classe M(3) de todas as possiveis tripartições de $I$ obtidas por duas partições dicotômicas $\gamma$ sucessivas, que é dada por :

$\Pi(3)=$

$U\{[\Psi(J(\alpha), \gamma) * L(\alpha)] \cup[J(\alpha) * \Psi(L(\alpha), \gamma)]\}$ $d \in \Psi(I, \gamma)$

Através de u uma partição de $\Pi(3)$ constrói-se a classe $\Pi(4)$ de todas as possiveis tetrapartiçzes de I, obtidas por três divisões dicotômicas $\gamma$, de maneira análoga à obtença da classe $\Pi(3)$.

As multipartiços subsequentes podem ser obtidas até se atingir a partição onde cada subconjunto tem apenas um elemento, ou seja 
A classe de todas as possiveis partiços do conjunto I, obtidas pela aplicação sucessiva de partiç̃o dicotómica $\gamma$ é indicada por $T(I, \gamma)$.

Fazendo $\Pi(1)=(1)$ e $\Pi(2)=\Psi(I, \gamma)$, tem-se

que

$$
T(I, \gamma)=\bigcup_{j=1}^{K} \Pi(j)
$$

Esses conceitos e o modo hierárquico de construção da classe $T(I, y)$ permitem uma analogia com um grafo terminal de dois, na teoria dos grafos, uma vez que um grafo consiste de um conjunto de nós e um conjunto de arcos. No presente caso, as partiços correspondem aos nos, $i \in T(1, \gamma 2$. Quanto aos arcos, diz-se que duas partif̧oes (nós) $i$ e $i$ de $T(I, \gamma)$ são adjacentes (arcos conectados se uma pode ser obtida da outra por uma partição dicotômica simples $\gamma$ de um dos seus subconjuntos.

2.3. Critérios de partição e regras de parada

Uma vez estabelecido o objetivo de se obterem grupos homogêneos internamente, é de interesse imediato saber que partiços levam ao agrupamento mais homogeneo.

Pode-se dizer que o problema é semelhante à classificaço cruzada múltipla em análise de variancia, uma vez que a técnica aglomerativa tenta agrupar caselas 
que têm médias muito próximas. Como cada casela é determinada de maneira única pelos niveis dos fatores, os grupos são estabelecidos atraves das relações entre a variável resposta e os fatores. Em BUSSAB (1976) existe uma discussão detalhada de um modelo apresentado em SONQUIST ${ }^{1}$ que, a seguir, é apresentado resumidamente.

$O$ conjunto de $N$ observações e formado por vetores na forma $\left(y, x_{1}, x_{2}, \ldots, x_{p}\right)$ onde $y$ corresponde à variável dependente $y e x_{1}, x_{2}, \ldots, x_{p}$ a um conjunto de fatores ou preditores $X_{1}, X_{2}, \ldots, X_{p}$. Os preditores são classificações, cada uma das quais com $k_{p}$ classes cou niveis), para $1 \leq p \leq P$.

Considerando todas as combinações possiveis dos níveis dos preditores em uma classificação total, sejam $K$ del as não vazias, com $n_{k}$ elementos, $1 \leq k \leq k$. Cada indice $k$ corresponde a uma única combinação dos niveis, podendo ser considerado, então, como um tratamento. Sendo cada um dos $N$ valores observados de $y$ um número de uma dessas caselas, então eles podem ser indicados por $y_{k j}$, representando o $j$-ésimo elemento da $k$-ésima casela, com $1 \leq k \leq K$ e $1 \leq j \leq n_{k}$. Para simplificar a notação, seja $y_{k}$ a média da k-ésima casela ou tratamento, ent $\widetilde{a}$

$$
y_{k}=\frac{1}{n_{k}} \sum_{j=1}^{n_{k}} y_{k j}
$$

1 SONQUIST,J.N. Multivariate Model Building. Harbor, Institute for Social Research, University of Michigan, 1970. 
Una medida de homogeneidade quando as $N$ observaçoes estão em um unico grupo e dada por

$$
\operatorname{saT}(\operatorname{total})=\sum_{k=1}^{k} \sum_{j=1}^{n_{k}}\left(y_{k j}-\bar{y}\right)^{2}
$$

onde $\bar{y}$ é a média geral. A quantidade SQTCtotal pode ser considerada, tambem, como uma medida de dispersão, uma vez que representa a variabilidade de todas as observafües em relaçăo à media geral $\bar{y}$. A expressão ( 2.9$)$ pode ser decomposta em duas somas de quadrados, SQE(total) e SQDCtotal) que são denominadas, respectivamente, variaçăo entre caselas e variaça dentro de caselas. Assim

$$
\begin{aligned}
& \operatorname{soT}(\operatorname{tatal})=\operatorname{SaE}(\operatorname{total})+\operatorname{soD}(\operatorname{total}) \\
& =\sum_{k=1}^{K} n_{k}\left(y_{k}-\bar{y}\right)^{2}+\sum_{k=1}^{k} \sum_{j=1}^{n}\left(y_{k j}-y_{k}\right)^{2} .
\end{aligned}
$$

A funçáo perda quadratica SoT(total) pode ser considerada como a perda de informaçáo que resulta de tratar todos os $N$ elementos como tendo a mesma média geral $\bar{y}$ Ja a quantidade sobrotaly é a perda de informaço por tratar cada observafá como a média da casela $k, y_{k}$. İ́ a quantidade soEctotcly é a perda de informaço por tratar cada média de casela cono a média geral ponderada pelo número de elementos em cada casela.

$$
\text { Com o objetivo de agrupar as } K \text { caselas em, }
$$
por exemplo, $G$ grupos, definem-se as seguintes expressoes 
onde $\sum_{k \in q}$ indica a soma dentre os tratamentos alocados ao g-ésimo grupo:

- $N_{g}=\sum_{k \in g} n_{k}:$ rúmero de el ementos no g-ésimo grupo.

$\cdot \bar{y}_{g}=\sum_{k \in g} n_{k} y_{k} / N_{g}:$ média do g-ésimo grupo.

$$
n_{k}
$$

- $\operatorname{SQT}\left(g\right.$ rupo) $=\sum_{k \in g} \sum_{j=1}^{k}\left(y_{k j}-\bar{y}_{q}\right)^{2}:$ soma de quadrados total no $\mathcal{E}^{-e ́ s i m o ~ g r u p o . ~}$

- $\operatorname{SQE}\left(\right.$ grupo) $=\sum_{k \in g} n_{k}\left(y_{k}-\bar{y}_{g}\right)^{2}$ : soma de quadrados entre caselas no g-ésimo grupo.

$n_{k}$

- $5 Q 0\left(\right.$ grupo) $=\sum_{k \in g} \sum_{j=1}^{k}\left(y_{k j}-y_{k}\right)^{2}:$ soma de quadrados

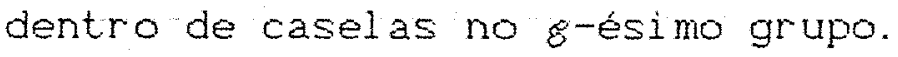

- $S Q R(G)=\sum_{g=1}^{a} \operatorname{SQE}(g r u p O)=\sum_{g=1}^{G} \sum_{k \in q} n_{k}\left(y_{k}-\bar{y}_{g}\right)^{2}:$ soma de quadrados residual devida aos $G$ grupos.

- 5 Qxe 0$)=\sum_{g=1}^{a} N_{g}(\bar{y}-\bar{y})^{2}:$ soma de quadrados devida aos G grupos.

De acordo com WARD (1963), sot $\operatorname{gorupo}_{\text {é a }}$ perda de informaça devido ao g-ésimo grupo e à perda de informação do modelo de $G$ grupos é a soma das perdas de cada grupo, isto é, 


$$
\sum_{g=1}^{G} S Q T_{g}(g r u p o) .
$$

Mostra-se facilmente que

$$
\begin{aligned}
\text { SQT }(\text { grupo }) & =\sum_{k \in g} \sum_{j=1}^{n_{k}}\left(y_{k j}-\bar{y}_{g}\right)^{2}= \\
& =\sum_{k \in g} \sum_{j=1}^{n_{k}}\left(\left(y_{k j}-y_{k}\right)+\left(y_{k}-\bar{y}_{g}\right)\right]^{2}= \\
& =\sum_{k \in g} \sum_{j=1}^{n_{k}}\left(y_{k j}-y_{k}\right)^{2}+\sum_{k \in g} n_{k}\left(y_{k}-\bar{y}_{g}\right)^{2}= \\
& =S Q E(g r u p O)+S Q D(g r u p o)
\end{aligned}
$$

e também

$$
\begin{aligned}
\sum_{g=1} S Q D(g r u p o) & =\sum_{g=1}^{a} \sum_{k \in g} \sum_{j=1}^{n_{k}}\left(y_{k j}-y_{k}\right)^{2}= \\
& \left.=\sum_{k=1}^{k} \sum_{j=1}^{n_{k}}\left(y_{k j}-y_{k}\right)^{2}=\operatorname{sonctotal}\right) .
\end{aligned}
$$

Em consequência, tem-se que à perdà de informação no modelo de $G$ grupos é composta por duas parcelas: a soma de quadrados residual devido aos $G$ grupos e a perda de informação por tratar cada observaçăo como a média da casela $k, y_{k}$, ou seja 


$$
\begin{aligned}
\sum_{g=1}^{a} \operatorname{SQT}(g r u p o) & =\sum_{g=1}^{a} \operatorname{SQE}(g r u p o)+\sum_{g=1}^{a} S Q D(g r u p o) \\
& =S Q R(G)+\text { SQDCtotal }) .
\end{aligned}
$$

Como para um conjunto de dados SQDC totals é fixado, a perda de informação no modelo de $G$ grupos depende apenas de SQR(G), isto é, da maneira pela qual as $K$ caselas são partidas em $G$ grupos. Pode-se mostrar também que

$$
\begin{aligned}
\operatorname{SOT}(\operatorname{total}) & =\sum_{g=1}^{a} \sum_{k \in g} \sum_{j=1}^{n_{k}}\left(y_{k j}-\bar{y}_{g}\right)^{2}+\sum_{g=1} N_{g}\left(\bar{y}_{g}-\bar{y}\right)^{2} \\
& =\sum_{g=1}^{a} S Q T(g r u p O)+\sum_{g=1}^{a} N_{g}(\bar{y}-\bar{y})^{2} \\
& =\operatorname{SQR}(G)+\operatorname{SQD}(\text { total })+\operatorname{SQX}(G)
\end{aligned}
$$

Comparando (2.14) com (2.10) obtem-se

$$
\text { SQE(total }=\text { SQR(G) }+ \text { SQX(G) }
$$

Assim ao se tentar minimizar a perda SQR(G), maximiza-se SQX(G) uma vez que SQE(total) e fixo.

Como não se pode construir uma partição mais fina aceitável das $N$ observaçós do que aquela definida para cada casela ou tratamento formando um grupo, a expressão (2.13) diz que qualquer conjunto de grupos de caselas da uma perda entre sqDototal) e SOT(total), correspondendo, respectivamente à partição mais fina com $K$ grupos e a mais rudimentar com um único 
grupo. Esta corresponde a "perda reduzida" SQRCG) variando entre zero e SOE(total).

Levando em conta essas premissas, o problema de análise da perda de informação considerando todos os elementos no g-ésimo grupo como tendo o mesmo valor $\bar{y}_{g}$, é equivalente aquele da análise da perda de informação considerando todas as $k$ médias de caselas no g-ésimo grupo tendo a mesma média $\vec{y}_{\mathrm{g}}$.

Considere a expressão SQR(G) como a perda da informação no modelo com $G$ grupos, e $S Q X(G)$ como a soma de quadrados explicada pelo modelo. Uma medida usada para dar informação sobre o modelo é a estatistica $k^{2}$, que expressa a proporcão da soma de quadrados explicada pelo modelo em relação à soma de quadrados total. No presente caso pode-se colocá-la como

$$
\left.R^{2}(G)=s Q \times(\theta), \text { sQTetotal }\right)
$$

Demonstra-se que

$$
0=R^{2}(1) \leq R^{2}(G) \leq R^{2}(K)<1
$$

onde, $R^{2} C K$ corresponde ao coeficiente de determinaça de um modelo com $k$ tratamentos. Uma forma reduzida no intervalo $[0,1]$ e dada por

$$
R_{*}^{2}(G)=\frac{\operatorname{sox}(\theta)}{\operatorname{soE}(\operatorname{tota} 2)}=\frac{R^{2}(G)}{R^{2}(k)} .
$$

Esta última pode ser interpretada como a proporcão da soma de quadrados explicada na constituiça de tais 
modelos. Entretanto a aplicação desse concei to especifico em cada partição torna-se trabalhosa devido ao grande número de elementos de $T(I, \gamma)$.

Um procedimento melhor para agrupar as médias em grupos homogêneos pode ser encontrar uma partição das caselas tal que :

- caso 1 : a partição tenha a menor $S Q R(G)$, para um número fixado $G$.

- caso 2 : a partição tenha o menor número de grupos $G$ para um nível fixado de $S Q R(G)$.

As ténicas de agrupamento têm sido aplicadas tanto no primeiro caso como no segundo, sendo que neste último surge a questão da escolha do valor mínimo desejado de perda de informação.

2. 4. O método de agrupamento divisivo hierárquico

Deseja-se encontrar uma partição otima de caselas tal que a função perda quadratica $S Q R$ seja minimizada sob certas restrições Como $o$ número de partiços possiveis das caselas e finito, pode-se encontrar uma partição calculando a função perda para cada partição e então escolhendo uma que satisfaça às condiçoes impostas. Esse procedimento é factivel para $G$ fixado desde que os valores de $K$ sejam pequenos.

No caso em que $G$ não é conhecido, é prudente restringir as tecnicas de análise de 
agrupamentos àquela que atue hierarquicamente, de modo que en cada estagio possa ser verificado se a perda minima é atingida ou outra regra de parada é alcançada.

o metodo de agrupamento hierárquico divisivo é um procedimento simples e fácil, composto pelos seguintes passos:

- No inicio todas as caselas estão em um único agrupamento. For algum procedimento otimo, esse grupo pode ser dividido em dois.

- Outro criterio de selecão escolhe um (ou os dois) dos grupos resultantes e se faz uma nova divisão.

- o passo anterior é repetido até que o número total de grupos finais é atingido ou alguma regra de parada é satisfeita.

Considerando que se tenha atingido uma particăo $d$, com $G$ grupos, o método divisivo hierárquico pode ser colocado matematicamente, como dividindo o 8-ésimo grupo em dols novos, $\varepsilon_{1}$ e $\varepsilon_{2}$. Verifica-se entzo que

$$
N_{g}=N_{g_{1}}+N_{g_{2}} \quad e \quad N_{g} \bar{y}_{g}=N_{g_{1}} \bar{y}_{g_{1}}+N_{g_{2}} \bar{y}_{g_{2}} .
$$

e que a perda de informaça sofre una reduça soE(d) definida por

$S Q E(d)=S Q R(G)-\operatorname{SQR}(G+1)$ 


$$
\begin{aligned}
& =S Q E(g r u p O)-S Q R_{g_{1}}(\text { grupo })-S Q R_{g_{2}} \text { (grupo) } \\
& =S Q X(G+1)-S Q X(G) \\
& =N_{g_{1}}\left(\bar{y}_{g_{1}}-\bar{y}\right)^{2}+N_{g_{2}}\left(\bar{y}_{g_{2}}-\bar{y}\right)^{2}-N_{g}(\bar{y}-\bar{y})^{2} .
\end{aligned}
$$

Donde se obtém

$$
\operatorname{SQE}(d)=N_{g_{1}} \bar{y}_{g_{1}}{ }^{2}+N_{g_{2}} \bar{y}_{g_{2}}{ }^{2}-N_{g} \bar{y}_{g}^{2} .
$$

No contexto de análise de variancia, a expressão (2.21) é a soma de quadrados entre tratamentos de dois grupos resultante de uma partição dicotomica do grupo ancestral bem como o montante de explicação ganho pela divisão.

A redução $S Q E(d)$, que muitas vezes é chamada apenas por redução $S Q E$, tem sido usada como base para o critério do método divisivo hierárquico.

Dessa maneira, dado que o método de agrupamento divisivo hierárquico foi aplicado, conseguiu-se uma partição formada por $G$ grupos de $I$, isto é

$$
I=\bigcup_{g=1}^{\mathrm{G}} I_{g} .
$$

Cada partição a de $\Psi$ CI ${ }_{g}, y$ tem associada a redução $S Q E(d)$ que é obtida, por exemplo, pela expressão (2.21). Seja BCI, ${ }_{g}$ ) o maximo entre as somas de quadrados entre grupos, isto $e$, 
$\left.B\left(I_{g}, \gamma\right)=\max \left(\operatorname{SQE}(\alpha): \alpha \in \mathbb{W} I_{g}, \gamma\right)\right\rangle$

A partição d tal que

$$
\operatorname{SQE}\left(\alpha_{0}\right)=B\left(I_{g}, \gamma\right)
$$

pode ser considerada a melhor divisão dicotômica.

Diversos autores, entre eles FISHER (1958) e SCOTT \& KNOTT (1974), para o caso de única classificação, HARTIGAN (1972) para o caso de classificação cruzada e Sonquist \& Morgan ${ }^{2}$ além de Sonquist et alii ${ }^{3}$ citados por BUSSAB (1976) para o caso de classificação múltipla cruzada propuseram diferentes maneiras de escolher o próximo grupo $G$ que será dividido, bem como parar o processo de divisão caso o número total de conjuntos na partição não seja especificado "a priori". De uma maneira geral, pode-se dizer que para a próxima divisão é escolhido o conjunto $I_{g}$ que conduz ao valor máximo de $B(I, \gamma)$ e como regra de parada do processo e verificado se a estatistica $B\left(I_{q}, \gamma\right)$ é significativa a um nível pré-fixado.

2 SONQUIST, J.N. \& MOEGAN, J. A. The Detection of Interaction Effects Monografia no. 35, Survey Research Center, Institute for Social Research, University of Michigan, 1904.

3 SONQUIST, J.H. ; BAKER, E. L. ; MORGAN, J. A. Searching for Structure. Institute for Social Research, University of Michigan, 1971. 
2.5. Criterios para a escolha do próximo grupo

A Iiteratura sobre técnicas divisivas de agrupamentos é muito extensa, mas poucos artigos tratam o problema especifico de agrupamento de médias em uma classificação múltipla como mencionado na seção anterior. Quatro deles são apresentados a seguir.

2.5.1. Agrupamento para homogeneidade máxima

As técnicas de agrupamento propostas por FISHER (1958) têm por objetivo formar grupos homogêneos para uma única variável. Seus resultados são importantes para esta discussão, apesar dele não ter usado o método de partição dicotomica hierárquica.

Nesse artigo e abordado o agrupamento com e sem restriçóes nas partições. Como exemplo do primeiro caso, tem-se a formação de grupos homogêneos em uma série temporal onde a ordem cronológica deve ser respeitada. Para o agrupamento sem restriçôes, tem-se como il ustraçăo a criaçăo de estratos homogêneos em amostragem.

Também é colocado que no caso da regra $\gamma_{L}$ e o conjunto $I$ ter $K$ elementos, a classe $\psi\left(1, \gamma_{L}\right.$ tera $e^{K-1}-1$ partiçóes. Neste caso para se encontrar a partição $d_{0} \in$ W $I, \gamma_{L}{ }^{2}$ com a maior reduça haverá um trabalho computacional muito grande. Entretanto, no artigo em questão é demonstrado que a solução está no subconjunto $\Psi I_{M}, \gamma_{M}$ onde $\gamma_{M}$ é a regra monotónica e $I_{M}$ é o conjunto 
ordenado pelos valores das médias das caselas ou tratamentos.

2.5.2. Técnica AID ("Automatic Interaction Detection")

\section{O algoritmo AID C"Automatic Interaction}

Detection") tem por propósito agrupar as caselas usando partição dicotómica nas médias marginais dos preditores em problemas de classificação múl tipla cruzada na análise de variancia e foi desenvolvida por Sonquist e Morgan ${ }^{4}$, de acordo com BUSSAB (1976).

O ganho otimo $B\left(\alpha, x_{p}\right)$ de um preditor $x_{p}$ é obtido através de suas médias marginais e utilizando a regra $\gamma_{\mathrm{p}}$ associada-a- ele.- 0 algoritmo escolhe como partição otima aquela que produz o maior valor de $B\left(d, X_{p}\right.$ ? , $1 \leq p \leq P$.

A regra de parada principal é baseada no conceito de ganho proporcional de uma nova divisão que é dado por

$$
R^{2}[d(\theta+1)]=R^{2}[d(\theta)]+\frac{\operatorname{SQE}[\alpha(\theta+1)]}{\operatorname{SoT}(\operatorname{total})}
$$$$
=\frac{\operatorname{sox}(206+1)]}{\operatorname{saT}(\operatorname{total})}
$$

4 SONOUIST, J.N. \& MORGAN, J.A. The detection of Interaction Effects. Monograph no. 35, Survey Research Center, Institute for Social Research. University of Michigan, 1964. 
onde sox $[d(G+1)]$ é a soma de quadrados devida aos grupos da nova partição d $(G+1)$. Sua significancia é baseada em resultados empíricos e, em geral, fixa-se o valor arbitrário 0,006, de acordo com SONOUIST \& MORGAN, citados por BUSSAB (1976), isto é, se o ganho relativo $S Q E\left(d, X_{p}\right)$, SQT (total) é menor que 0,006 o processo pára.

Posteriormente, novas versões do algoritmo foram sugeridas, considerando al gumas opções e diferentes alternativas de análise, mas a essência do algoritmo é a mesma, segundo EUSSAB (1976).

2.5.3. Agrupamento de casos e variáveis

O algoritmo proposto por HARTIGAN (1972) para agrupar casos e variáveis corresponde na realidade a uma classificação dupla com uma resposta por casela. A essência desse algoritmo é a mesma da técnica AID.

Diz-se que uma familia de grupos é uma árvore se dois grupos quaisquer não se sobrepõem. Esses dois grupos são também disjuntos ou um inclui o outro. 0 agrupamento de Hartigan é feito de tal modo que a estrutura de arvore é alcançada não só nas caselas bem como nas médias marginais. Tem-se, então, uma regra complexa $\gamma$.

o processo se resume em encontrar um elemento de $T(1, \gamma)$ através da técnica de agrupamento divisivo hierárquico que reduz a soma de quadrados a um valor razoável. Em cada passo tal estatistica é calculada e o processo para quando seu valor esperado é maior que o 
obtido. Aqui também o conhecimento da distribuição da variavel $B(I, y)$ é fundamental, apesar de, em geral, ela ser desconhecida.

Considerando um conjunto de variáveis aleatorias independentes, $y_{1}, y_{2}, \ldots, y_{K}$, e $I=$ $(1,2, \ldots, k)$ um conjunto de indices, bem como $\gamma_{\mathrm{L}}$ uma regra 11 vre de partiçôes, a expressão (2. 23 ) pode ser reescrita como

$B\left(I, \gamma_{L}\right)=$

$$
=\max \left\{\frac{y_{(1)}+\ldots+y_{(p)}}{p}-\frac{y_{(p+1)}+\ldots+y_{(k)}}{k-p}\right\} \frac{p(k-p)}{k}
$$

(2. 26$)$

onde $y_{(k)}$ é a k-ésima média ordenada. Sob a hipotese nula $y_{k}, 1 \leq k \leq x$, tem uma distribuiçăo $N\left(\mu, \sigma^{2}\right)$. FISHER (1958) usou o resultado de que para $K$ grande a partição otima sempre ocorre ao redor de $\mathrm{p}=0,5$, isto é,

$$
\left|\mathrm{p}_{0}-0,5\right|=0\left(\mathrm{~K}^{-0,5}\right)
$$

onde $p_{o} e$ e ponto onde ocorre a divisão. Com essa informaça o autor provou que assintoticamente

$$
\left.B C I, \gamma_{L}\right) \sim e \sigma^{2} \chi_{(K / \pi)}^{2}
$$

onde, $x_{(k, \pi)}^{2}$ significa uma variavel aleatoria qui-quadrado com $k / \pi$ graus de 1 iberdade.

Nesse artigo existem expressöes assintóticas para a média e a variancia de $B C I, \gamma_{L}$. BUSSAB 
(1976) obteve, entretanto resultados diferentes usando o mesmo método.

2.5.4. Teste de máxima verossimilhança

- método de agrupamento divisivo hierárquico foi usado por SCOTT \& KNOTT (1974) para agrupar medias de tratamentos quando um teste $F$ na análise de variância rejeita a hipótese de que elas são iguais, ou seja, para o caso de uma classificação com número igual de elementos por casela e com a regra $\gamma$ livre para divisão dicotómica.

Uma estatistica $\lambda$ da razão de máxima verossimilhança é construida para testar a igualdade de todas as médias contra a alternativa de que elas podem ser divididas em dois grupos desconhecidos. Para cada amostra esse grupo é dado pela particão $d_{0} \in \Psi\left(I, \gamma_{L}{ }^{2}\right.$ tal que $\left.\operatorname{soE}\left(\alpha_{0}\right)=B C I, \gamma_{L}\right)$. Aplicando a estatistica de teste $\lambda$ tem-se a regra de parada do procedimento divisivo.

Posteriormente BUSSAB (1976) deduziu o teste da razão de máxima verossimilhança para o caso de qualquer tipo de regra $\gamma$, e caselas com diferentes números de elementos.

De uma maneira geral, têm-se as seguintes suposições:

- As $K$ médias de caselas $y_{1}, y_{2}, \ldots, y_{K}$ são variáveis aleatorias independentes com $y_{k} \sim N\left(\mu_{k}, \sigma^{2} / n_{k}\right)$, para $k=1,2, \ldots, k$. 
$\nu s^{2} / \alpha^{2} \sim \chi^{2} v$

- Uma regra $\gamma$ é fixada para a obtenção da divisão dicotômica.

- O resultado do teste estatístico da analise de variancia rejeita a hipotese de igualdade de médias das caselas e existem evidéncias de que elas possam ser divididas em dois grupos homogêneos distintos.

Então para $I=(1,2, \ldots, k)$, qualquer partiçăo $d \in \Psi(I, \gamma)$ implicara que a media dos tratamentos e dada por

$$
\mu_{k}= \begin{cases}m_{J} & \text { se } k \in J(d) \\ m_{L} & \text { se } k \in L(d)\end{cases}
$$

no espaco de parametros $\left(\mu_{1}, \mu_{2}, \ldots, \mu_{K}\right)$. Assim o espaço de parametros desse vetor de médias e determinado unicamente por

$$
\psi(I, \gamma) \cup\left\{d_{*}\right\}
$$

onde, $d_{*} \in$ a partiçăo $\langle 1, \emptyset)$ que corresponde ao caso onde todas as médias são iguais.

Então sob a hipótese nula

$$
\left.H_{0}: \mu_{k}=\mathrm{m}, k \in I \text { Correspondendo a } d_{*}\right)
$$


- logaritmo da função de verossimilhança

$L(m, \alpha)=-(k+\nu) \log \sigma-\frac{1}{2 \alpha^{2}}\left\{\nu s^{2}+\sum_{k \in I} n_{k}\left(y_{k}-m\right)^{2}\right\}$

assume seu valor máximo em

$$
\left.L \bar{y}, \sigma_{0}\right\rangle=\operatorname{cte}-\left(\frac{x+\nu}{e}\right)\left\{1+208 \sigma_{0}^{2}\right\}
$$

quando

$$
\begin{aligned}
& N=\sum_{k \in I} n_{k}, \quad \bar{y}=\sum_{k \in I} n_{k} y_{k} / N e \\
& \sigma_{0}^{2}=\frac{1}{k+v}\left\{v s^{2}+\sum_{k \in I} n_{k}\left(y_{k}-\bar{y}\right)^{2}\right\} .
\end{aligned}
$$

Sob a hipótese al ternativa

$$
H_{1}: \mu_{k}=\left\{\begin{array}{l}
m_{J} \text { se } k \in J(d), \\
m_{L} \text { se } k \in L(d),
\end{array}\right.
$$

para algum $\alpha \in \Psi(I, \gamma)$ onde $m_{J}$ e $m_{L}$ são as médias desconhecidas de dois grupos, o valor máximo do logaritmo da função de verossimilhança

$$
\left.L C \bar{y}_{J}, \bar{y}_{L}, \sigma_{1}\right)=\operatorname{cte}-\frac{k+\nu}{2}\left\{1+208 \sigma_{1}^{2}\right\}
$$


29.

$\sigma_{i}^{2}=\frac{1}{K+\nu}\left\{\nu s^{2}+\sum_{k \in J\left(d_{0}\right)} n_{k}\left(y_{k}-\bar{y}_{J}\right)^{2}+\sum_{k \in L\left(d_{0}\right)} n_{k}\left(y_{k}-\bar{y}_{L}\right)^{2}\right\}$

onde

$$
\begin{aligned}
& N_{J}=\sum_{J(d, j} n_{k}, N_{L}=\sum_{L\left(d_{0}\right)} n_{k}, \\
& \bar{y}_{J}=\sum_{J i d, k} n_{k} y_{k} / N_{J}, \bar{y}_{L}=\sum_{L\left(d_{0}\right)} n_{k} y_{k} / N_{L},
\end{aligned}
$$

sendo $\sum_{\left.J<d_{0}\right)}$ a soma para todo $k \in J\left(d_{0}\right)^{2} \sum_{L\left(d_{0}\right)}$ a soma para todo $k \in L\left(d_{0}\right)$ e $d_{0} e$ o elemento de $\Psi(I, \gamma)$ que minimiza (2. 37).

Para se obter a estatistica do teste da razão de verossimilhança de $H_{0}$ vs $H_{1}$ basta calcular a diferença entre (2. 36) e (2.33), isto é,

$$
-\frac{k+v}{2} \log \left(\sigma_{1}^{2}, \sigma_{0}^{2}\right)
$$

e rejeita-se $H_{0}$ para valores grandes dessa expressão. Mostra-se que esse procedi mento é equi valente a rejeitar = $H_{\text {o }}$ para valores grandes de

$$
1-\frac{\sigma_{1}^{2}}{\sigma_{0}^{2}}=\frac{N_{J} \bar{y}_{J}^{2}+N_{L} \bar{y}_{L}^{2}-N \bar{y}^{2}}{v s^{2}+\sum_{k \in I} n_{k}\left(y_{k}-\bar{y}\right)^{2}}
$$




$$
1-\frac{\sigma_{1}^{2}}{\sigma_{0}^{2}}=\frac{B(I, \gamma)}{\nu s^{2}+\sum_{k \in I} n_{k}\left(y_{k}-\bar{y}\right)^{2}}
$$

O resultado (e.41) corresponde à estatistica para se testarem as mesmas hipóteses, obtida por SCOTT \& KNOTT (1974) no caso de mesmo número de observaçōes por casela, ou seja $n_{k}=N / k$, $\forall k \in I$, e $\gamma=$ $\gamma_{L}$. Usando o resultado de HARTIGAN (1972) da expressão (2.28), eles mostraram que a variável

$$
\lambda=\frac{\pi}{\partial(\pi-2)} \times \frac{B\left(I, \gamma_{L}^{3}\right.}{\sigma_{0}^{2}}
$$

e assintoticamente equivalente a uma variável qui-quadrado com $k /(\pi-2)$ graus de liberdade. Usando simulaçăo, foi mostrado que qui-quadrado é uma boa aproximação para valores pequenos ou moderados de $v / K$, mas năo para valores grandes.

No caso em que a variancia ó ${ }^{2}$ é conhecida ou $v \rightarrow \infty,(2.41)$ torna-se

$$
\frac{B(I, \gamma)}{\sigma^{2}}
$$

que é usada tanto para o teste com grandes amostras como quando a variancia é conhecida.

No próximo capitulo será apresentada uma breve introduçáo aos modelos lineares generalizados para que posteriormente alguns resultados aqui apresentados possam ser estendidos. 


\section{UMA INTRODUÇÃO AOS MODELOS LINEARES GENERALIZADOS}

Um grande número de métodos estatísticos tais como modelos de regressão, modelos log-lineares, dentre outros, podem ser considerados como casos particulares de uma classe mais ampla de modelos que foi introduzida por NELDER \& WEDDERBURN (1972) e é conhecida como modelos ineares generalizados (GLM). O texto clássico para o estudo de tais modelos, McCULLAGH \& NELDER (1989), serviu de base para este capítulo que faz uma breve introdução aos modelos lineares generalizados para poder ser definida uma medida mais geral de grupos homogêneos.

3.1. O ajuste de um modelo

Ao se considerar uma relação li near entre duas variáveis, por exemplo, $y$ e $x$, o ajuste de uma reta do tipo $y=a+b x$, pressupõe a escolha de um par especifico $(a, b)$ de um conjunto de todos os pares possiveis de valores de parametros que tornam o conjunto de valores preditos, $\hat{y}_{1}, \hat{y}_{2}, \ldots, \hat{y}_{n}$, o mais próximo possivel dos valores observados. A fim de tornar o conceito de proximidade mais objetivo, e importante definir uma medida de distancia ou discrepancia entre os valores 
observados e os valores ajustados. Tanto a norma $L_{1}$, dada por

$$
s_{1}(y, \hat{y})=\sum_{i}\left|y_{i}-\hat{y}_{i}\right|
$$

como a norma $L_{\infty}$, dada por

$$
s_{\infty}(y, \hat{y})=\max _{i}\left|y_{i}-\hat{y}_{i}\right|
$$

são exemplos de funções de discrepância. No ajuste de mínimos quadrados, a medida de discrepancia usada é a norma $L_{2}$ ou a soma de quadrados dos desvios, que é dada por

$$
s_{2}(y, \hat{y})=\sum_{i}\left(y_{i}-\hat{y}_{i}\right)^{2}
$$

Essa abordagem de discrepancia tem duas consequencias. A primeira refere-se à soma direta dos desvios individuais, tanto em $\left|y_{i}-\hat{y}_{i}\right|$ como em $\left(y_{i}-\hat{y}_{i}\right)^{2}$, onde, cada um desses desvios depende apenas de uma observaçăo, implicando que as observaçöes são todas feitas na mesma escala física e sugere que as observaçães são independentes ou, pelo menos, que elas sejam de algum modo permutáveis, justificando entăo um manuseio dos componentes. A segunda esta relacionada com o fato de que o uso das diferencas aritméticas $y_{i}-\hat{y}_{i}$ implica em que todos os desvios têm pesos iquais independente do valor $\hat{y}$. Na terminologia estatistica, a utilidade da norma $L_{p}$ como medida de discrepancia depende da independencia estocástica bem como da suposiça de que a variancia de 
cada observação é independente do seu valor médio. Tais suposiços, embora comuns e frequentes na prática, não têm sentido em aplicaçóes mais gerais.

Outra funçăo que pode ser adotada como medida de discrepancia tem por base estimativas obtidas pela maximização da funç̃o de verossimilhança. Seja $f(y ; \theta)$ a função densidade de probabilidade para uma distribuição de parâmetro $\theta$. O logaritmo da função de verossimilhança expresso como uma funçăo do parametro $\mu=E(Y)$ é dado por

$$
l(\mu ; y)=\ln f(y ; \theta)
$$

e no caso de um conjunto de $n$ observaçóes independentes $y_{1}, \ldots, y_{n}$ é a soma das contribuiçöes individuais,

$$
l(\underset{y}{\mu}, y)=\sum_{i=1}^{n} \text { ln } f_{i}\left(y_{i} ; \theta_{i}\right)
$$

onde $\mu_{n}=\left(\mu_{1}, \ldots, \mu_{n}\right)$

A medida de discrepancia utilizada no ajuste de um modelo I inear generalizado e a funça desvio ("scaled deviance") que é dada por

$$
D(y ; \mu)=2[2(y ; y)-2(\mu ; y)]
$$

onde, ly;y) e o logaritmo da funcăo de verossimilhança calculado no ponto onde os valores ajustados sâ iguais às observaçöes e $(2 \mu, y)$ como em (3.5).

Para modelos de regressão linear da teoria em que os erros têm distribuiç̃o normal com variancia 
conhecida $\sigma^{2}$, tem-se, para uma unica observação, a função densidade

$$
f(y ; \mu)=\left(2 \pi \sigma^{2}\right)^{-1 / 2} \exp \left[-(y-\mu)^{2} /\left(2 \sigma^{2}\right)\right]
$$

e o logaritmo da função de verossimilhança,

$$
l(\mu ; y)=\frac{1}{2} \ln \left(2 \pi \sigma^{2}\right)-\frac{(y-\mu)^{2}}{2 \sigma^{2}} .
$$

Para $\mu=y$ obtém-se o valor máximo do logaritmo da função de verossimilhança resultando

$$
2(y ; y)=-\frac{\ln \left(2 \pi \sigma^{2}\right)}{2} .
$$

Obtém-se então a função desvio que é dada por

$$
D^{*}(y ; \mu)=2(2(y ; y)-2(\mu ; y)\}=\frac{(y-\mu)^{2}}{\sigma^{2}}
$$

Esta expressão é identica à soma de quadrados dos resíduos, a menos da constante conhecida $\sigma^{2}$. Aqui desvio míni mo esinónimo de mínimos quadrados.

Os modelos lineares generalizados são uma extensão dos modelos lineares clássicos, e seu ajuste é feito pelo método de máxima verossimilhança. 
3.2. Os componentes de um modelo linear generalizado

Seja uma amostra aleatória $\underset{\sim}{Y}=\left(Y_{1}, \ldots, Y_{n}\right)^{\prime}$ com média $\mu_{\sim}=\left(\mu_{1}, \ldots, \mu_{n}\right)$ cujos elementos têm uma distribuição que pertence à familia exponencial de distribuiçőes, ou seja,

$$
f(y ; \theta, \phi)=\exp \left\{\frac{y \theta-b(\theta)}{\alpha(\phi)}+c(y, \phi)\right\}
$$

onde $E(Y)=\mu=b^{\prime}(\theta)$ e $\operatorname{Var}(Y)=\alpha(\phi) V$ com $V=b^{\prime \prime}(\theta)=\mathrm{d} \mu / \mathrm{d} \theta$ denominada função de variancia, é expressa em termos da média $\mu$. O parâmetro natural, $\theta=\int \nu^{-1} d \mu$, $\Leftrightarrow$ uma função de $\mu$, isto é, $\theta=q(\mu)$ e caracteriza a distribuição. Geralmente $\alpha(\phi)=\phi / \psi$, sendo $\phi$ interpretado como um parâmetro de dispersão, e $w$ como peso "a priori". A familia exponencial pode ser de dois parametros se $\phi$ for desconhecido.

Entre as médias das observações e a estrutura linear do modelo, existe uma função de ligação $g($.$) de tal modo que g(\mu)=\eta=x \beta$, onde $\eta=\left(\eta_{1}, \ldots, \eta_{n}\right)^{\prime}$ chamado preditor linear, é uma função linear dos $k$ parametros desconhecidos $\beta_{\alpha}=\left(\beta_{1}, \ldots, \beta_{k}\right), e \&($.$) , suposta$ conhecida, é diferencíavel Em geral $g 6$ a não élinear e $X$ é uma matriz do modelo conhecida. A caracterização de um modelo linear generalizado é feita, então, através da especificação de três componentes. O primeiro refere-se ao componente aleatório, que é determinado pela indicaçăo de $E(Y)=\mu$ eda $\operatorname{Var}(Y)$. O segundo refere-se ao componente sistemático que produz o preditor linear $\eta$. O terceiro componente é a especificação da função de ligação 8 C. . , 
que relaciona o componente aleatorio ao componente sistemático.

Todas as vezes em que $\theta=\eta$, onde $\theta$ é a função $q(\mu)$, diz-se que a ligação utilizada é a canónica.

No modelo linear clássico a média, $\mu$, , e o preditor linear, $\eta$, săo identicos. A funçăo de ligaçăo é a identidade, que nesse caso coincide com a canónica.

Em modelos log-lineares para contagens baseados na independencia de dados de classificação cruzada a função de ligaçăo utilizada é a logaritmica, que nesse caso é a canónica.

Para a distribuiça binomial, tem-se que $0<\mu<1$ e a ligação devem satisfazer a condição de levar o intervalo $(0,1)$ à reta real. As funções mais utilizadas รััo :

- Logística

$$
n=\ln \{\mu /(1-\mu)\}
$$

que é a ligação canônica para o modelo de erro binomial.

- Probito

$$
\eta=\Phi^{-1}(w)
$$

onde T. $\in$ a funçăo de distribuição acumulada normal.

- Complemento log-log

$$
\eta=\ln [-\ln (1-\mu)]
$$


A familia potencia de funções de ligação também é muito utilizada e pode ser especificada de duas maneiras. A primeira através de

$$
\eta=\left\{\begin{array}{l}
\left\langle\mu^{\lambda}-1\right\rangle \lambda \text { para } \lambda \neq 0 \\
\ln \mu \text { quando } \lambda=0
\end{array}\right.
$$

e a segunda por

$$
\eta=\left\{\begin{array}{l}
\mu^{\lambda} \text { se } \lambda \neq 0 \\
\ln \mu \text { se } \lambda=0
\end{array}\right.
$$

Em MCCULLAGH \& NELDER (1989, p. 30 ) existe uma tabela que resume as principais caracteristicas de distribuições univariadas da familia exponencial. A função de ligação apresentada é a ligação canónica para a qual existe uma estatistica suficiente dada por $X^{\prime} \underset{\sim}{y}$, com dimensão igual à dimensão de $\beta$, no preditor linear $\eta=X \beta$ As ligaçôes canónicas conduzem a propriedades estatisticas desejáveis no modelo, principalmente em amostras pequenas. Entretanto não existe, em geral, uma razão "a priori" no modelo que exija isso. Em cada caso a função de ligação também deve ser estudada na adequabillidade do modelo.

3. 3 Inferencias sobre o modelo linear general izado

Ao se ajustar um modelo a um conjunto de dados, é de interesse saber quão discrepantes estão os 
valores observados, $y$, em relaça aos valores ajustados, $\hat{\mu}$, obtidos a partir de um modelo que, em geral, envolve um número $\rho$ pequeno de parametros. Como mencionado na Seção 3.1., a medida de discrepancia ou ajuste de um modelo pode ser obtida de acordo com vários critérios, entre eles o de máxima verossimilhança, que corresponde a duas vezes o logaritmo de uma razão de verossimilhanças, a qual foi chamada de função desvio em (3.6).

Com relação ao número de parametros, diversos modelos podem ser ajustados a um conjunto de observaçós. O mais simples, o modelo nulo, tem apenas um parâmetro, representando a mesma média para todas as observaçöes; pode-se dizer que esse modelo contém toda a variação dos $y^{\prime \prime} s$ devido ao componente aleatório. No outro extremo o modelo completo tem $n$ parâmetros, um por observaç̃o, e os $\mu^{\prime} s$ correspondem a cada uma das observaçóes, ficando toda a variaçăo dos y's para o componente sistemático.

Apesar desses dois modelos serem ou muito simples ou não informativos, eles servem de base para se medir a discrepancia em um modelo intermediário com $p$ parâmetros.

Sejam $\langle(\hat{\mu}, \phi ; y)$ o logaritmo da funcão de verossimilhança maximizado sobre $\beta$ para um valor fixado do parametro de dispersão, $\phi$, e $\langle(y, \phi ; y)$ o logaritmo da função de verossimilhança maximizado em um modelo completo com $n$ parâmetros. A discrepancia de um ajuste é proporcional a duas vezes a diferença entre o logaritmo da verossimilhanca maximizada em um modelo completo com $n$ parametros e o logaritmo da função de verossimilhança maximizado sobre $\beta$ para um valor fixado do parametro de 
dispersão, $\phi$, no modelo sob pesquisa. Se $\hat{\theta}=\theta(\hat{\mu})$ e $\tilde{\theta}=\theta(y)$ são as estimativas dos parâmetros canónicos sob os dois modelos, a discrepância, assumindo $\alpha_{i}(\phi)=\phi / w_{i}$ pode ser escrita por

$$
\begin{aligned}
D^{*}(y ; \hat{\mu})= & 2\left[l(\chi ; \chi)-l\left(\hat{\sim}_{i} ; y\right)\right]= \\
= & 2 \sum_{i=1}^{n}\left\{\left[\left(y_{i} \tilde{\theta}_{i}-b\left(\tilde{\theta}_{i}\right\rangle\right] / \alpha_{i}(\phi)+c\left(y_{i}, \phi\right)\right]-\right. \\
& -\left[\left(y_{i} \hat{\theta}_{i}-b\left(\hat{\theta}_{i}\right) 3 / \alpha_{i}(\phi)+c\left(y_{i}, \phi\right)\right]\right\} \\
= & \sum_{i=1}^{n} 2 w_{i}\left\{y_{i}\left(\tilde{\theta}_{i}-\hat{\theta}_{i}\right)-\left[b\left(\tilde{\theta}_{i}\right)-b\left(\hat{\theta}_{i}\right)\right]\right\} / \phi \\
= & D(y ; \hat{\omega}) / \phi
\end{aligned}
$$

onde, $D(y ; \hat{\mu})$, chamado de desvio ("deviance") para o modelo sob estudo, é uma função apenas dos dados. Vale reforçar, também, que a função desvio $D^{*}(y ; \hat{\mu})$, definida em $(3.6)$ e desvio dividido pelo parâmetro de dispersão. $\phi$.

Os graus de liberdade associados ao desvio $s$ ão definidos por $\nu=n-p$, onde $n$ e o número de observaçōes e $\rho$ é o número de parámetros 1 i nearmente independentes no modelo.

Para o caso de distribuição normal, o desvio coincide com a soma de quadrados do residuo, enquanto que para a distribuição de Poisson, ele é a estatistica $G^{2}$ mencionada por BISHOP et alii (1975).

Outra importante medida de discrepancia é 
uma generalização da estatistica de Pearson que e dada por

$$
x^{2}=\sum_{i=1}^{n}\left(y_{i}-\hat{\mu}_{i}\right)^{2} N\left(\hat{\mu}_{i}\right)
$$

onde, $V\left(\hat{\mu}_{i}\right)$ é a função de variância estimada para a distribuiçăo sob estudo. Para a distribuição normal, $x^{2}$ também coincide com a soma de quadrados dos resíduos, enquanto que para as distribuiçós de Poisson e binomial obtem-se a estatistica $X^{2}$ de Fearson original.

Tanto o desvio como a estatistica de Pearson $x^{2}$ generalizada têm distribuição proporcional a uma distribuiçăo de qui-quadrado no caso de erros com distribuiçăo normal, desde que o modelo assumido seja verdadeiro. A vantagem do desvio sobre $x^{2}$ como medida de discrepancia é que ele é aditivo para conjuntos de modelos hierárquicos se as estimativas de máxima verossimilhança são usadas, enquanto que $x^{2}$, em geral, não o é. Entretanto é mais fácil interpretar $x^{2}$ do que o desvio.

Saindo da teoria de modelos lineares com distribuiçăo normal para os erros, a distribuição do desvio ainda năo foi estabelecida. Fara as distribuições exponencial e normal inversa, resultados exatos ja foram obtidos, quando as observaçós provêm de um delineamento simples.

Uma maneira de se testar o modelo sob estudo é através de uma estatistica expressa pelo quociente 


$$
R=\frac{\left(D_{1}-D_{p}\right)<(p-1)}{D_{p} /(n-p)}
$$

onde $D_{1}$ e o desvio de um modelo com um parametro e $D_{\mathrm{p}}$, - desvio do modelo de interesse com p parâmetros. Essa estatistica pode ser aproximada por uma distribuicão $F$ com $(p-1)$ e $(n-p)$ graus de liberdade, de acordo com JORGENSEN (1983).

Tabela 3 - Quadro de analise do desvio para um modelo fatorial completo com dois fatores principais.

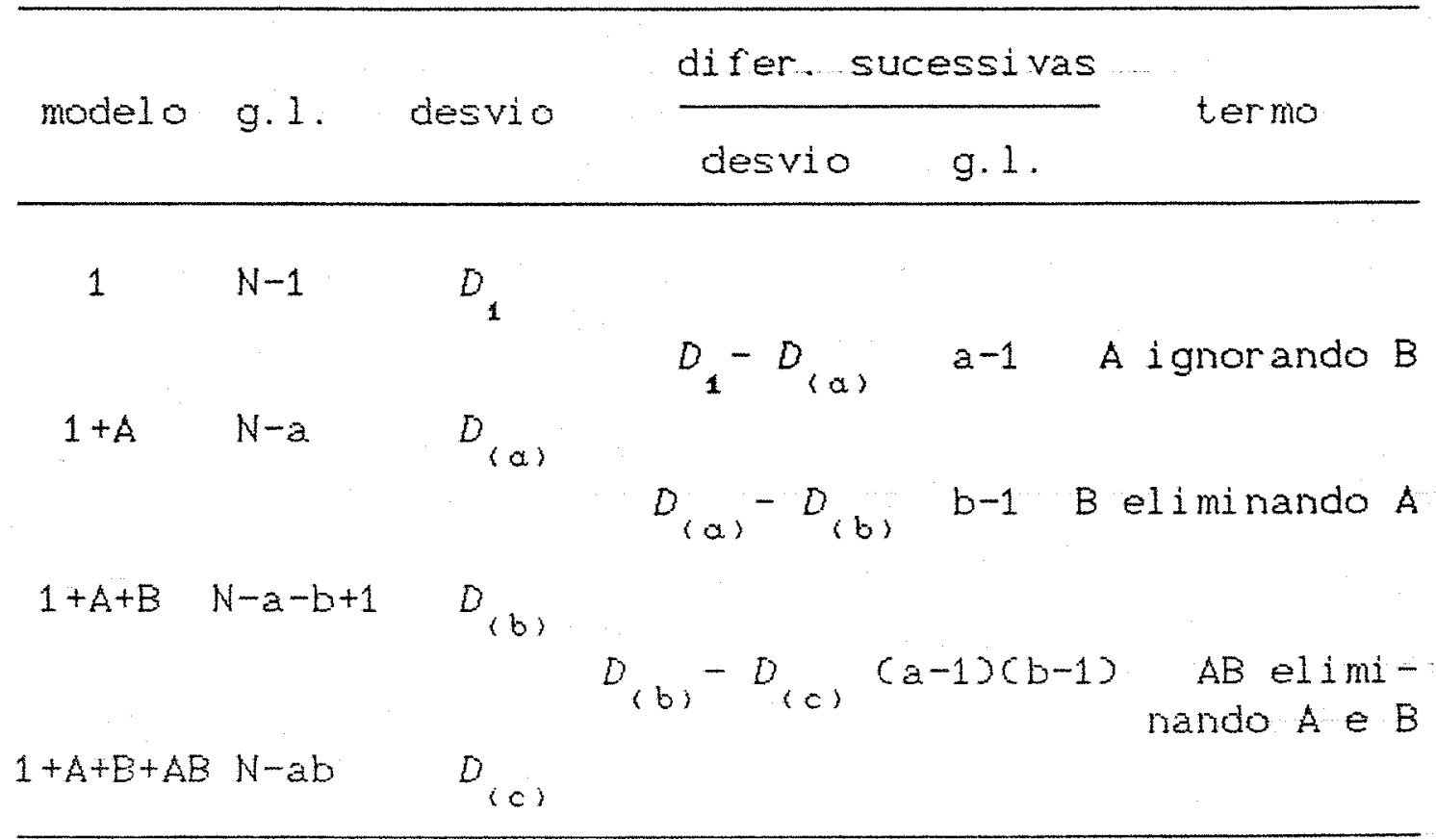

Nota: $(a)=1+A,(b)=1+A+B,(c)=1+A+B+A B$

De uma maneira análoga à feita em análise de variancia pode-se construir uma tabela de análise de desvio. As diferenças de desvios e correspondentes graus de 1 iberdade fornecem a reduçăo no desvio com a adição de cada termo sucessivo e generaliza a analise de variancia 
sequencial para modelos lineares. Por exemplo, para um modelo fatorial com dois fatores, uma tabela de analise do desvio com 3 termos $A, B$ e a interaçăo $A B$ esta na Tabela 3. Se os dados provêm de uma distribuição normal, o desvio corresponde à soma de quadrados dos respectivos modelos. As diferenças sucessivas de desvios e graus de liberdade fornecem elementos para compor os numeradores de quocientes do tipo da expressão (3.19) que são usadas para testar os respectivos termos.

Os resíduos săo tambem definidos para modelos lineares generalizados, obtendo-se, segundo o critério de definição geral dos residuos, os de Pearson, os de Anscombe e os componentes do desvio.

A verificação da adequabilidade do modelo ("model checking") constitui uma área da estatistica que ultimamente teve seu desenvolvimento acelerado devido, principalmente, às facilidades computacionais existentes no mercado. Um texto básico para a verificação da adequabilidade do modelo e o de ATKINSON (1985) e uma resenha mais recente desse assunto pode ser encontrada em, por exemplo, ATKINSON et alii (1989).

Diversos trabalhos tratam da extensão de técnicas de diagnóstico para o caso de ML ou modelos de regressão generalizados. Entre eles estão os trabalhos de PIERCE \& SCHAFER (1986), WILLIAMS (1987), WANG (1987), LEE (1988) e DAVISON (1989a). Devido a certas peculiaridades da distribuiçăo binomial, os trabalhos de PREGIBON (1981), FOWLKES (1987) e DAVISON (1989b) tratam de técnicas de diagnosticos para dados binarios.

Para a verificação da adequabilidade da função de 1 igação têm-se, por exemplo, os trabalhos de 
PREGIBON (1980) e ATKINSON (1982).

3.4. O algoritmo de estimação

- algoritmo proposto por NELDER \& WEDDERBURN (1972) para ajuste de modelos lineares generalizados corresponde à obtenção de estimativas de máxima verossimilhança dos parâmetros $\beta$ no preditor linear $\eta$ por minimos quadrados ponderados iterativos.

Seja $\hat{\eta}^{(0)}$ a estimativa inicial do preditor Inear que corresponde ao valor ajustado $\hat{\mu}^{(0)}$ obtido a partir da função de 1 lgação $n=g(\mu)$. Seja $z_{\sim}^{(0)}$ a variavel dependente ajustada definida por

$$
z_{i}^{(0)}=\hat{\eta}_{i}^{(0)}+\left(y_{i}-\hat{\mu}_{i}^{(0)}\right)\left(\frac{d \eta}{d \mu}\right)^{(0)}
$$

onde a derivada da função de ligação e calculada en $\hat{\mu}^{(0)}$. Seja, também, o peso quadrático definido por

$$
\left[w_{i}^{(0)}\right)^{-1}=\left\{\left(\frac{d \eta_{i}}{d \mu_{i}}\right]^{(0)}\right]^{2} v^{(0)}
$$

onde $v_{i}^{(0)} \in$ a função de variancia calculada em $\hat{\mu}_{i}^{(0)}$. Agora faz-se a regressão de $\underset{\sim}{(0)}$ sobre as covariáveis $x_{1}, x_{2}, \ldots, x_{p}$ com peso $w^{(0)}$ para se obterem as novas estimativas $\hat{\beta}^{(1)}$ dos parametros, dessa forma obtém-se uma nova estimativa de $\hat{\eta}^{(1)}$, do preditor linear. Repete-se o 
processo ate que as mudancas sejam minimas.

A variavel $z$ uma forma linearizada da função de ligação aplicada aos dados, ou seja,

$$
g(y) \simeq g(\mu)+(y-\mu) g^{\prime}(\mu) .
$$

Como ponto de partida recomenda-se o valor $\hat{u}^{\langle 0\rangle}=y$, que, em geral, faz com que a convergencia do algoritmo seja mais rápida.

o algoritmo de ajuste utilizado é uma variante do metodo de Newton-Raphson, conhecido como método de escore.

Devido às suas características especiais, um sistema de ajuste iterativo de modelos lineares generalizados conhecido como GLIM ("Generalised Linear Interative Modelling System") foi desenvolvido na Inglaterra pelo grupo NAG ("Numerical Algorithms Group Ltd"). A edição aqui utilizada é a 3.77 (PAYNE,1986). 
4. UMA MEDIDA MAIS GERAL DE HOMOGENEIDADE DE GRUPOS E ALGUMAS CONSEQUENCI AS

\subsection{Introdução}

Para a classe da familia exponencial, uma estatística baseada no desvio é proposta como medida de homogeneidade de grupos.

Além do desvio minimo para o modelo de grupos, uma generalização de estatisticas do tipo $R^{2}$ é deduzida para auxiliar na adaptação do algoritmo de agrupamento divisivo hierárquico nos casos das distribuições normal, Poisson e bi nomial.

o desenvol vimento de um teste da razão de verossimilhança para o caso geral é apresentado com o objetivo de generalização do procedimento de SCOTT \& KNOTT (1974).

4.2. Uma extensão natural para a medida de homogeneidade de grupos

Para a classe de modelos que pertence à familia exponencial na forma (3.11), pode-se utilizar como medida de discrepância no seu ajuste 


$$
\begin{aligned}
& D(\underset{z}{z} ; \hat{\sim})=\phi D^{*}(\underset{z}{y} ; \hat{\sim})= \\
& =\sum_{i=1}^{n} 2 w_{i}\left[y_{i}\left(\tilde{\theta}_{i}-\hat{\theta}_{i}\right)-b\left(\tilde{\theta}_{i}\right)+b\left(\hat{\theta}_{i}\right)\right]
\end{aligned}
$$

obtida a partir de (3.17) para $\alpha_{i}(\phi)=\phi / w_{i}$, e que corresponde à soma de quadrados de residuos no modelo normal equiponderado. Quanto menor o desvio, a discrepáncia ou o residuo, mais bem ajustado está o modelo.

$\mathrm{Na}$ situação especifica de comparações de caselas pode-se dizer que quanto maior o desvio, mais heterogenneas são as caselas. Se o objetivo é encontrar uma medida de homogeneidade de grupos de caselas, o desvio entre dois grupos de caselas pode, então, ser usado.

Considere o modelo de um ensaio completamente ao acaso de $K$ caselas ou tratamentos com $n_{k}$ repetições por casela. 0 desvio do modelo associado, $\left.D C y ; \hat{\mu}_{\sim+K}\right)$, pode ser expresso por

$$
\begin{aligned}
& \left.\left.D C y ; \hat{\mu}_{1+K}\right\rangle=\sum_{k=1}^{K} \sum_{j=1}^{n_{k}} e w_{k j}\left[y_{k j}\left(\tilde{\theta}_{k j}-\hat{\theta}_{k}\right)-b\left(\tilde{\theta}_{k j}\right)+b c \hat{\theta}_{k}\right)\right]
\end{aligned}
$$

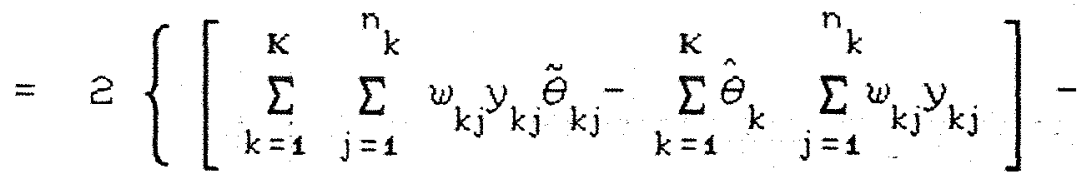

$$
\begin{aligned}
& \left.-\left[\sum_{k=1}^{k} \sum_{j=1}^{n_{k}} w_{k j} b\left(\tilde{\theta}_{k j}\right)-\sum_{k=1}^{k} b C \hat{\theta}_{k}, \sum_{j=1}^{n_{k}} w_{k j}\right]\right\}
\end{aligned}
$$


onde, $\left.\tilde{\theta}_{k j}=\theta<y_{k j}\right)$ e $\left.\hat{\theta}_{k}=\theta<\hat{\mu}_{k j}\right)$. O número de graus de liberdade associado a $(4.2)$ e $n-k, \operatorname{com} n=\sum_{k=1}^{K} n_{k}$. O desvio do mesmo ensaio considerando todas as médias iguais entre si, $D\left(y ; \hat{\sim}_{1}\right)$, tem $n-1$ graus de liberdade e pode ser expresso por

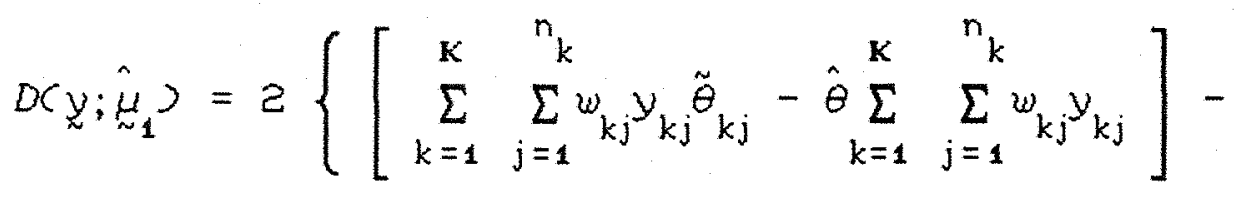

$$
\begin{aligned}
& \left.-\left[\sum_{k=1}^{k} \sum_{j=1}^{n_{k}} w_{k j} b\left(\tilde{\theta}_{k j}\right)-b(\hat{\theta}) \sum_{k=1}^{k} \sum_{j=1}^{n_{k}} w_{k j}\right]\right\}
\end{aligned}
$$

onde, $\tilde{\theta}_{k j}=\theta\left(y_{k j}\right)$ e $\hat{\theta}=\theta(\hat{\mu})$.

Pela expressão ( 3.19 ), a razão

$$
R=\frac{\left[D\left(y ; \hat{\mu}_{1}\right)-D\left(y ; \hat{\mu}_{\sim 1+K}\right)\right] /(K-1)}{D\left(y ; \hat{\mu}_{1+K}\right) /(n-K)}
$$

pode ser usada para se testar a igual dade de médias das caselas uma vez que possui, assintoticamente, distribui $̧$ ão $F(K-1, n-K)$ sob $H_{o}$, segundo JORGENSEN (1983).

Considerando o caso especifico de partiçăc dicotómica do conjunto de índices $I=\{1,2, \ldots, \mathrm{K}\}$, segundo a regra $\gamma$, cada partição a forma dois conjuntos de indices de caselas constituidos por $\mathcal{J}(d)$ e $L(\alpha)$. Seja; entao, $D\left(y ; \hat{\mu}_{d}\right)$ o desvio do modelo de dois grupos definidos pela partição d. A razão 


$$
\frac{D\left(y ; \hat{\mu}_{1}\right)-D\left(y ; \hat{\mu}_{d}\right)}{D\left(y ; \hat{\mu}_{d}\right) /(n-2)}
$$

pode ser usada para se testar a hipótese de igualdade de médias de dois grupos pré-fixados, quando comparada com uma distribuição $F$ com 1 e n-2 graus de liberdade, tendo por base, tambem, a expressão (3.19).

A diferença no numerador de $(4.5)$ é uma medida de homogeneidade de dois grupos, uma vez que $D\left(y ; \hat{u}_{d}\right)$ é uma medida de discrepancia do modelo de dois grupos.

Usando a expressão $(4.1)$, sendo $\hat{\theta}_{i}=\theta\left(\hat{\mu}_{d}\right)$, tal que $\theta\left(\hat{\mu}_{d}\right)=\hat{\theta}_{\mathrm{J}(\mathrm{d})}$ quando a 1 -ésima casela pertence ao conjunto $J(d)$ e $\theta\left(\hat{\mu}_{d}\right)=\hat{\theta}_{L(d)}$, quando ao conjunto $L(d), \cdots$ desvio $D\left(y ; \hat{\mu}_{d}\right)$ pode ser expresso por:

$$
\begin{aligned}
& D\left(y ; \hat{\mu}_{d}\right)=2\left\{\sum_{k=1}^{k} \sum_{j=1}^{n_{k}} w_{k j}\left[y_{k j} \tilde{\theta}_{k j}-b<\tilde{\theta}_{k j}\right)\right]- \\
& -\left[\sum_{J(d)} \sum_{j=1}^{n_{k}} w_{k j}\left[y_{k j} \hat{\theta}_{J(d)}-\delta\left\langle\hat{\theta}_{J(d)}\right\rangle\right]+\right. \\
& \left.+\sum_{L\langle d\rangle} \sum_{j=1}^{n_{k}} w_{k j}\left[y_{k j} \hat{\theta}_{L i d\rangle}-b\left(\hat{\theta}_{L(d\rangle}\right)\right]\right\}
\end{aligned}
$$

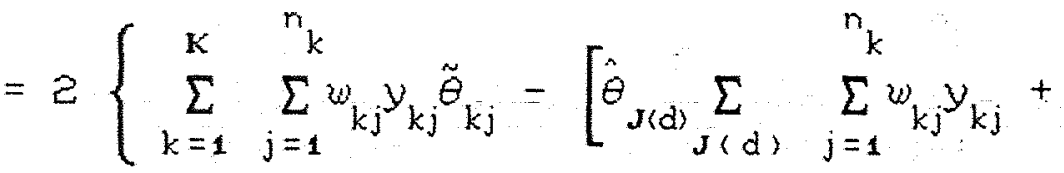

$$
\begin{aligned}
& \left.+\hat{\theta}_{L(d)} \sum_{L(d)} \sum_{j=1}^{n_{k}} w_{k j} y_{k j}\right]-\left[\sum_{k=1}^{K} \sum_{j=1}^{n_{k}} w_{k j} \delta c \tilde{\theta}_{k j}\right\rangle-
\end{aligned}
$$


49.

$$
-\left[\delta\left\langle\hat{\theta}_{J(d)} \sum_{J(d)} \sum_{j=1}^{n_{k}} w_{k j}+\delta\left(\hat{\theta}_{L(d)}>\sum_{L(d)} \sum_{j=1}^{n_{k}} w_{k j}\right]\right]\right\}
$$

onde, $\Sigma$ refere-se à soma das caselas que pertencem a $J(d)$

$J(\alpha)$ e $\Sigma$, à soma das caselas que estão no conjunto $L(\alpha)$.

Quanto menor o desvio $D\left(y ; \hat{\mu}_{d}\right)$ mais heterogeneos serão os dois grupos formados pela partição d. Assim para se obter a partição que produz os dois grupos mais heterogeneos entre si, basta encontrar aquela que produz o menor desvio. Como $D\left(y ; \hat{\mu}_{1}\right)$ é fixo para qualquer partição $d$, uma vez que representa o desvio de um modelo com apenas uma média para todas as caselas, a diferença

$$
\Delta\left(\hat{\mu}_{d}\right)=D\left(y ; \hat{\mu}_{\sim_{1}}\right)-D\left(y ; \hat{\mu}_{d}\right)
$$

pode ser utilizada, também, como medida de homogeneidade de dois grupos de caselas, e nesse caso quanto maior a diferença (4.7) mais heterogeneos serão os dois grupos.

A partir das expressões (4.3) e (4.6), a medida de homogeneidade dos dois grupos, de caselas definida em (4.7) pode ser reescrita por:

$$
\begin{aligned}
& \Delta\left(\hat{\mu}_{d}\right)=D\left(y ; \hat{\mu}_{1}\right)-D\left(y ; \hat{\mu}_{d}\right)= \\
& =e\left\{\left[\hat{\theta}_{J(d)} \sum_{J\langle d\rangle} \sum_{j=1}^{n_{k}} w_{k j} y_{k j}+\hat{\theta}_{L\langle d\rangle} \sum_{L\langle d\rangle} \sum_{j=1}^{n_{k}} w_{k j} y_{k j}-\right.\right.
\end{aligned}
$$




$$
\begin{aligned}
& \left.-\hat{\theta} \sum_{k=1}^{K} \sum_{j=1}^{n_{k}} w_{k j} y_{k j}\right]-\left[b \left(\hat{\theta}_{J(d)}>\sum_{J(d)} \sum_{j=1}^{n_{k}} w_{k j}^{+}\right.\right. \\
& \left.+\delta\left(\hat{\theta}_{L(d)} \sum_{L(d)} \sum_{j=1}^{n_{k} w_{k j}}-\partial(\hat{\theta}) \sum_{k=1}^{K} \sum_{j=1}^{n_{k}} w_{k j}\right]\right\}
\end{aligned}
$$

A diferença $\Delta\left(\hat{\mu}_{\sim}\right)$ usada como uma medida de homogeneidade de dois grupos formados pela partição das $K$ caselas, possui formas diferentes para cada distribuição, conforme será exposto nas próximas seçðes.

\subsection{O caso normal}

A expressão (4.1) representa o desvio para qualquer membro da familia exponencial. Na distribuição normal o parâmetro de dispersão $\Leftrightarrow \phi=\sigma^{2}, b(\theta)=\theta^{2} / 2$ e a funçăo de ligação canónica é a identidade. Considerando que $w_{k j}=1$, o desvio para um ensaio completamente casualizado com $k$ tratamentos ou caselas e $n_{k}$ repetiços por casela, dado em (4.2), torna-se

$$
\begin{aligned}
D\left(y ; \hat{\mu}_{1+k}\right)= & e\left\{\left[\sum_{k=1}^{k} \sum_{j=1}^{n_{k}} y_{k j}^{2}-\sum_{k=1}^{k} \bar{y}_{k} \sum_{j=1}^{n_{k}} y_{k j}\right]-\right. \\
& \left.-\left[\sum_{k=1}^{k} \sum_{j=1}^{n_{k}}\left(y_{k j}^{2}, e\right)-\sum_{k=1}^{k}\left[n_{k}\left(\bar{y}_{k}^{2}-e\right)\right]\right]\right\} \\
= & \sum_{k=1}^{k} \sum_{j=1}^{n}\left(y_{k j}-\bar{y}_{k}\right)^{2}
\end{aligned}
$$


51.

que corresponde à soma de quadrados do resíduo do referido modelo, uma vez que $\tilde{\theta}_{k j}=\tilde{\mu}_{k j}=y_{k j}$ e $\hat{\theta}_{k}=\hat{\mu}_{k}=\bar{y}_{k}$.

De maneira análoga, o desvio de um modelo de um ensaio completamente casualizado com $K$ caselas e $n_{k}$ observações por casela sob a hipotese de que todas as medias de caselas são iguais, dada em (4.3), torna-se a soma de quadrados total. Assim a expressão (4.4) no caso normal, representa a razão utilizada para se testar o efeito de média de caselas no modelo de um ensaio completamente ao acaso, que sob a hipótese nula tem distribuição $F(K-1, n-K)$.

Com relação ao desvio (4.6), que é uma medida de discrepância obtida pela partição $d$, tem-se que

$$
D\left(y ; \hat{\mu_{j}}\right)=\sum_{k=1}^{k} \sum_{j=1}^{n_{k}} y_{k j}^{2}-n_{J(d)} \hat{y}_{J(d)}^{2}-n_{L(d)} \bar{y}_{L(d)}^{2}
$$

uma vez que

$$
\begin{aligned}
& \tilde{\theta}_{k j}=\hat{\mu}_{k j}=y_{k j}, \\
& \hat{\theta}_{J(d)}=\hat{\mu}_{J(d)}=\bar{y}_{J(d)}=\sum_{J(d)} n_{k} \bar{y}_{k} / n_{J(d)}, \\
& \text { - } \hat{\theta}_{L(d)}=\hat{H}_{L(d)}=\bar{y}_{L(d)}=\sum_{L(d)} n_{k} \bar{y}_{k} / n_{L(d)}, \\
& \bar{y}_{k}=\sum_{j=1} y_{k j} / n_{k}, \\
& \text { - } n_{J(d)}=\sum_{J(d)} n_{k}, \\
& \text { - } n_{L(d)}=\sum_{L(d)} n_{k} .
\end{aligned}
$$


A expressão (4.10) corresponde à soma de quadrados de residuos de um modelo com $k$ caselas de $n_{k}$ observações cada uma, que pela partição a nos indices produziu duas médias para cada grupo de caselas.

Quanto à diferença de desvios (4.8), no modelo com distribuição normal obtém-se

$$
\Delta\left(\hat{\beta}_{d}\right)=n_{J(d)} \bar{y}_{J(d)}^{2}+n_{L(d)} \bar{y}_{L(d)}^{2}-n \bar{y}^{2}
$$

A expressão (4.11) corresponde à soma de quadrados entre dois grupos formados pela partição $d$ de $K$ caselas. Comparando (2.21) e (4.11), verifica-se que a diferença $\Delta\left(\hat{\mu}_{d}\right)$ reduz-se a $S Q E(\alpha)$, quando o grupo ancestral 8 tem como média $\bar{y}$.

\subsection{O caso Poisson}

Na. distribuiça de Poisson têm-se $\phi=1$ e $b(\theta)=\exp (\theta)$. No caso de um ensaio completamente ao acaso com $k$ caselas e $n_{k}$ repetiços por casela, peso "a priori" $w_{k j}=1$, o desvio, considerando a função de ligação a natural, isto é, a logaritmica, torna-se

$$
D\left(y ; \hat{\mu}_{1+k}\right\rangle=e\left\{\sum_{k=1}^{k} \sum_{j=1}^{n_{k}} y_{k j} \ln \left(y_{k j}\right)-\sum_{k=1}^{k} n_{k} \bar{y}_{k} \ln \left(\bar{y}_{k}\right\rangle\right\}
$$

onde, 
- $\tilde{\theta}_{k j}=\ln \left(\hat{\mu}_{k j}\right)=\ln \left(y_{k j}\right) e$

- $\hat{\theta}_{k}=\ln \left(\hat{\mu}_{k}\right)=\ln \left(\bar{y}_{k}\right)$.

De maneira análoga, $D\left(y ; \hat{\mu}_{i}\right)$ representa 0 desvio do ensaio completamente ao acaso, sob a hipótese de que todas as médias de caselas são iguais. Com a distribuição de Poisson, ele pode ser escrito por

$$
D\left(y ; \hat{\mu}_{1}\right)=2\left[\sum_{k=1}^{k} \sum_{j=1}^{n_{k}} y_{k j} \ln \left(y_{k j}\right)-n \bar{y} \ln (\bar{y})\right]
$$

onde,

$$
\begin{aligned}
& \cdot \tilde{\theta}_{k j}=\ln \left(\hat{\mu}_{k j}\right)=\ln \left(y_{k j}{ }^{2} e\right. \\
& \cdot \hat{\theta}=\ln (\hat{\mu})=\ln (\bar{y}) .
\end{aligned}
$$

Assim a expressão (4.4) no caso Poisson representa a razão

$$
\begin{aligned}
R=\left\{\left[\sum_{k=1}^{K} n_{k} \bar{y}_{k} 2 n\left(\bar{y}_{k}\right)-n y \ln (\bar{y})\right] /(k-1)\right\} / \\
\left\{\left[\begin{array}{ccc}
k & n_{k} \\
\sum_{k=1} & \sum_{j=1} y_{k j} & \left.\left.2 n\left(y_{k j}\right)-\sum_{k=1}^{k} n_{k} \bar{y}_{k} \ln \left(\bar{y}_{k}\right)\right] / 6 n-k\right)
\end{array}\right\}\right.
\end{aligned}
$$

que tem distribuiçăo assintótica $F(K-1, n-k)$ sob a hipótese de igualdade de médias de caselas. 
medida de discrepância obtida pela partição d, tem-se que

$$
\begin{aligned}
& D\left(y ; \hat{\mu}_{d d}\right)=e\left\{\sum_{k=1}^{k} \sum_{j=1}^{n_{k}} y_{k j} \ln \left(y_{k j}\right)-\right. \\
& \left.-n_{J(d)} \bar{y}_{J(d)} \ln \left(\bar{y}_{J(d)}\right)-n_{L(d)} \bar{y}_{L(d)} \ln \left(\bar{y}_{L(d)}\right)\right\}
\end{aligned}
$$

onde,

$$
\begin{aligned}
& \cdot \hat{\theta}_{J(d)}=\ln \left(\hat{\mu}_{J(d)}\right)=\ln \left(\bar{y}_{J(d)}\right) e \\
& \cdot \hat{\theta}_{L(d)}=\ln \left(\hat{\mu}_{L(d)}\right)=\ln \left(\bar{y}_{L(d)}\right) .
\end{aligned}
$$

A expressão (4.15) e, então, a discrepancia medida em termos do desvio de um modelo de um ensaio completamente ao acaso onde as médias das caselas formam dois grupos definidos pela partição $d$, no caso da distribuição de Poisson.

Quanto à diferença de desvios, $\Delta\left(\hat{\mu}_{\mathrm{d}}\right)$, definida em ( 4.8$)$, tem-se

$$
\begin{aligned}
\left.\Delta C \hat{\mu}_{d d}\right\rangle=e & {\left[n_{J\langle d\rangle} \bar{y}_{J(d)} \ln \left\langle\bar{y}_{J(d)}\right\rangle+\right.} \\
& \left.+n_{L(d)} \bar{y}_{L(d)} \ln \left(\bar{y}_{L(d)}\right\rangle-n \bar{y} \ln (\bar{y}\rangle\right]
\end{aligned}
$$

4.2.3. O caso binomial 
55.

sobre dados binários, mesmo em MCCULLAGH \& NELDER (1989), considera a variável y como o número de sucessos.

Assim, sendo $y_{1}, \ldots, y_{n}$ os valores observados de variáveis aleatórias $y_{1}, \ldots, y_{n}$, onde $y_{i}$ tem distribuição binomial de indice $m_{i}$ e parâmetro $\pi_{i}$, logaritmo da função de verossimilhança pode ser escrito por

$$
\begin{aligned}
2\left(\pi_{\sim}, y\right) \propto & \sum_{i=1}^{n}\left[y_{i} \ln \left(\pi_{i} /\left(1-\pi_{i}\right)\right)+m_{i} \ln \left(1-\pi_{i}\right)\right] \\
& =\sum_{i=1}^{n}\left[y_{i} \ln \pi_{i}+\left(m_{i}-y_{i}\right) \ln \left(1-\pi_{i}\right)\right]
\end{aligned}
$$

A função desvio, que na distribuiçăo binomial tem a mesma notação do desvio, é dada por

$$
\begin{aligned}
D(y ; \hat{\pi}) & =2[2(\tilde{\pi} ; y)-2(\hat{\pi}, y)] \\
& =2 \sum_{i=1}^{n}\left\{y_{i} \ln \left(y_{i} / \hat{\mu}_{i}\right)+\left(m_{i}-y_{i}\right) \ln \left(\left(m_{i}-y_{i}\right) /\left(m_{i}-\hat{\mu}_{i}\right)\right)\right\}
\end{aligned}
$$

onde, $\hat{\mu}_{i} \Leftrightarrow$ o valor predito no modelo em questão.

Em um ensaio completamente ao acaso com $K$ caselas e $n_{k}$ repetiçóes, seja $y_{k j}$ o número de sucessos da j-ésima repetição da k-ésima casela, que possui distribuição binomial com indice $m_{k j}$ e parametro $\pi_{k j}$. 0 logari tmo da função de verossimilhança é dado por 


$$
l\left(\pi_{i} ; y^{\prime}\right)=\sum_{k=1}^{k} \sum_{j=1}^{n_{k}}\left[y_{k j} \ln \pi_{k j}+\left(m_{k j}-y_{k j} \sin \left(1-\pi_{k j}\right)\right]\right.
$$

que atinge seu máximo quando $\tilde{\pi}_{k j}=y_{k j} / m_{k j}$, obtendo-se então

$$
\begin{aligned}
\langle\hat{n} ; y\rangle= & \sum_{k=1}^{K} \sum_{j=1}^{n_{k}}\left[y_{k j} \ln y_{k j}+\right. \\
& \left.+\left(m_{k j}-y_{k j}\right) \ln \left(m_{k j}-y_{k j}\right)-m_{k j} \ln m_{k j}\right]
\end{aligned}
$$

Sob a hipótese de que o parámetro $\pi_{k j}$ é o mesmo para todas as repetições da mesma casela, obtém-se, de (4.19)

$$
\left\langle\left(\pi_{\sim K+1} ; y\right)=\sum_{k=1}^{K} \sum_{j=1}^{n_{k}}\left[y_{k j} \ln \pi_{k}+\left(m_{k j}-y_{k j}\right) \ln \left(1-\pi_{k}\right)\right]\right.
$$

(4.21)

que atinge seu máximo, considerando a função logito, 'em

$$
\hat{\pi}_{k}=\bar{y}_{k}=\left(y_{k .} / m_{k .}\right)=\left[\sum_{j=1}^{n_{k}} y_{k j}\right] /\left[\sum_{j=1}^{n_{k}} m_{k j}\right]
$$

e como valor esperado para cada observação

$$
\hat{\mu}_{k j}=m_{k j} \bar{y}_{k}
$$


é dada por

$$
\left\langle\left(\hat{\pi}_{\mathrm{K}+1} ; y\right)=\sum_{k=1}^{K}\left[y_{k} \ln \bar{y}_{k}+\left(m_{k}-y_{k}\right) \ln \left(1-\bar{y}_{k}\right)\right] .\right.
$$

Dessa maneira, para o modelo de um ensaio completamente ao acaso com $K$ caselas e $n_{k}$ observações por casela, sendo $y_{k j} \circ$ número de sucessos na j-ésima observação da k-ésima casela uma variável aleatória com distribuição binomial de indice $m_{k j} e$ parâmetro $\pi_{k}$, a função desvio dada em (4.18) torna-se

$$
\begin{aligned}
\left.D C y ; \hat{\pi}_{1+k}\right)= & =\left\{\sum _ { k = 1 } ^ { k } \sum _ { j = 1 } ^ { n _ { k } } \left[y_{k j} \ln y_{k j}+\right.\right. \\
& \left.+\left(m_{k j}-y_{k j}\right) \ln \left(m_{k j}-y_{k j}\right)-m_{k j} \ln m_{k j}\right]- \\
- & {\left.\left[\sum_{k=1}^{k}\left[y_{k} \ln \bar{y}_{k}+\left(m_{k}-y_{k}\right) \ln \left(1-\bar{y}_{k}\right)\right]\right]\right\} }
\end{aligned}
$$

Já para o modelo em que todas as caselas possuem 0 mesmo parametro $\pi$, o logaritmo da função de verossimilhança dado em (4.19), torna-se

$$
\begin{aligned}
\left\langle\left(\pi_{\sim_{1}} ; y\right)\right. & =\sum_{k=1}^{k} \sum_{j=1}^{n_{k}}\left[y_{k j} \ln \pi+\left(m_{k j}-y_{k j}\right) \ln (1-\pi)\right] \\
& =y \ldots \ln \pi+(m \ldots-y \ldots \ln (1-\pi)
\end{aligned}
$$


58.

onde, $y=\sum_{k=1}^{k} \sum_{j=1}^{n_{k}} y_{k j}$ e ${ }^{m}=\sum_{k=1}^{k} \sum_{j=1}^{n_{k j}} m_{k j}$ Seu maximo é atingido para

$$
\hat{n}=\bar{y}=y / m
$$

quando a função de ligação e logito, e o valor esperado para cada obser vação é dado por

$$
\hat{\mu}_{k j}=m_{k j} \bar{y}
$$

Assim a função desvio do modelo completamente casualizado, sob a hipótese de que todas as médias de caselas são iguais é dada por

$$
\begin{aligned}
& \left.D C_{x ; \pi_{1}}\right\}=2\left\{\sum _ { k = 1 } ^ { k } \sum _ { j = 1 } ^ { n } \left[y_{k j} \ln y_{k j}+\left(m_{k j}-y_{k j}\right) \ln \left(m_{k j}-y_{k j}\right)-\right.\right. \\
& \left.\left.-m_{k j} \ln m_{k j}\right]-\left[y \ldots \ln \bar{y} \ldots+c m^{-y} \ldots \ln (1-\bar{y})\right]\right\} \\
& \text { (4.29) }
\end{aligned}
$$

Considerando o modelo completamente casualizado sob a hipótese de que as $K$ médias de caselas formam dois grupos $J(d)$ e $L(d)$, definidos pela partição $a$ do conjunto de indices $I=\{1,2, \ldots, k\}, 0$ logaritmo da função de verossimilhança e dado por

$$
i\left(\pi_{d}, y\right)=\sum_{J(d)} \sum_{j=1}^{n_{k}}\left[y_{k j} l n \pi_{L(d)}+\left(m_{k j}-y_{k j}\right) 2 n\left(1-\pi \pi_{J(d)}\right)\right]+
$$




$$
+\sum_{L(d)} \sum_{j=1}^{n_{k}}\left[y_{k j} 2 n \pi_{L(d)}+\left(m_{k j}-y_{k j}\right) \ln \left(1-\pi_{L(d)}\right)\right]
$$

Seu máximo é atingido quando

$$
\begin{aligned}
& \hat{\pi}_{J(d)}=\sum_{J(d)} y_{k} \cdot / \sum_{J\langle d\rangle} m_{k}=\bar{y}_{J(d)} e \\
& \hat{\pi}_{L(d)}=\sum_{L(d)} y_{k} \cdot / \sum_{L(d)} m_{k .}=\bar{y}_{L(d)}
\end{aligned}
$$

obtendo-se então

$$
\begin{aligned}
& \left\langle\left(\hat{\pi}_{d} ; y\right\rangle=\right. \\
& =\left[\ln \bar{y}_{J(d)} \sum_{J(d)} y_{k}+\left[\sum_{J\langle d\rangle}\left(m_{k}-y_{k}\right)\right] \ln \left(1-\bar{y}_{J\langle d\rangle}\right)\right]+ \\
& +\left[\ln \bar{y}_{L(d)} \sum_{L(d)} y_{k .}+\left[\sum_{L(d)}\left(m_{k .}-y_{k}\right)\right] \ln \left(1-\bar{y}_{L(d)}\right)\right]
\end{aligned}
$$

o valor esperado de cada observação é, então, dado por

$\hat{\mu}_{k j}=m_{k j} \bar{y}_{J(d)}$, se $k \in J(d)$ e $\hat{\mu}_{k j}=m_{k j} \bar{y}_{L(d)}$, se $k \in L(\alpha)$.

A funçăo desvio do modelo definido pela partição dé dada por

$\left.D x_{y} ; \hat{\pi}_{d}\right\}=$ 
60.

$$
\begin{gathered}
=e\left\{\sum_{k=1}^{k} \sum_{j=1}^{n_{k}}\left[y_{k j} i n y_{k j}-6 m_{k j}-y_{k j}\right) \ln \left(m_{k j}-y_{k j}\right)-\right. \\
\left.\quad-m_{k j} \ln m_{k j}\right]-\left[\ln \bar{y}_{J(d)} \sum_{J(d)} y_{k}+\right. \\
+\ln \left(1-\bar{y}_{J(d)}\right) \sum_{J(d)}\left(m_{k}-y_{k .}\right)+\ln \bar{y}_{L(d)} \sum_{L(d)} y_{k}+ \\
+\ln \left(1-\bar{y}_{L(d)} \sum_{L(d)}\left(m_{k},-y_{k .}\right)\right]
\end{gathered}
$$

A função desvio (4.18) e suas decorrentes, tém o mesmo significado que a soma de quadrados de resíduos em modelos lineares ordinários. A adição de mais covariáveis no modelo tem o efeito de reduzir a função desvio.

Em diversas situações, a variável aleatoria definida em (4.18) pode ser considerada distribuída assintoticamente como uma variável qui-quadrado com $n-\rho$ graus de liberdade, onde $\rho$ é número de parametros independentes sob $H_{0}$.

Para se justificar tal fato usam-se duas suposições cuja relevância em qual quer apl icação deve ser verificada. A primeira delas refere-se à independéncia entre as observações da distribuição binomial, descartando a possibilidade de existir super dispersão. A segunda suposição diz respeito à aproximação que se faz em uma operação limite na qual $\operatorname{dim}(\underset{\sim}{ })=n \in$ fixada, $m_{i} \rightarrow \infty$ para cada $i$, e no fato de que $m_{i} \pi_{i}\left(1-\pi_{i}\right) \rightarrow \infty$.

Nesta última, a funçăo desvio é aproximadamente independente dos parametros estimados $\hat{\beta}_{\sim} e$ 
por essa razão aproximadamente independente das probabilidades ajustadas $\hat{\pi}_{n}$. Independência aproximada $\Leftrightarrow$ essencial à înção desvio considerada como uma estatistica de ajuste do modelo, mas essa propriedade sozinha não garante um bom poder.

Se $n$ e grande e $m_{i} \pi_{i}\left(1-\pi_{i}\right)$ permanece limitada, toda essa teoria falha em dois aspectos: a aproximação de qui-quadrado 1 imitante não mais acontece; e, o que é mais importante, a função desvio não é independente de $\hat{\pi}$ mesmo aproximadamente. Como consequência, um valor grande da função desvio pode ser obtido pela escolha de $\beta e \underset{\sim}{\pi}$. Em outras palavras um valor grande da função desvio pode não ser necessariamente considerado como uma evidência de um ajuste pobre. Em situações onde existe escassez ("sparseness", , $P_{i}$ pequeno) esse efeito surge com grande freqúencia.

Por essas razóes a função desvio não e utilizada diretamente como uma medida absoluta do ajuste do modelo, mas sim, para comparar dois modelos encaixados. Sendo $H_{0} 0$ modelo sob teste e $H_{\text {a }}$ o modelo estendido que contém uma covariável adicional, a redução na função desvio

$$
D\left(y ; \hat{\mu}_{0}\right)-D\left(y ; \hat{\mu}_{\alpha}\right)=e\left[2\left(\hat{\mu_{a}} ; y\right)-2\left(\hat{\mu}_{\alpha} ; y\right)\right]
$$

e idêntica à estatística do teste da razão de verossimilhança para testar $H_{o}$ contra $H_{a}$ Essa estatistica é distribuida assintoticamente como uma variável qui-quadrado com um grau de liberdade e independente de $\hat{\mu}_{\sim}$ sob a primeira suposicăo anteriormente 
citada desde que $n$ seja suricientemente grande ou que a segunda suposição esteja satisfeita. Em particular, $D\left(\underset{\sim}{y} ; \hat{\mu}_{0}\right)$ não precisa ter distribuição aproximadamente qui-quadrado e nem precisa ser distribuida independentemente de $\hat{\mu}_{0}$.

Por essas razoes, em vez de se construir uma estatistica semelhante a (4.4), para a hipótese de igual dade de médias, deve-se preferir a diferença

$$
\begin{aligned}
& D\left(y ; \hat{\pi}_{1}\right)-D\left(y ; \hat{\pi}_{1+K}\right)= \\
& =2\left\{\sum_{k=1}^{K}\left[y_{k .} \ln \bar{y}_{k}+\left(m_{k}-y_{k}\right) \ln \left(1-\bar{y}_{k}\right)\right]-\right. \\
& -[y . \ln \bar{y} \ldots+c m \ldots-y .2 \ln (1-\bar{y} .,]]\}
\end{aligned}
$$

que tem distribuição assintótica qui-quadrado com $(K-1)$ graus de liberdade.

Para uma partição particular $d, a$ diferença $\Delta\left(\hat{\sim}_{d}\right)$ definida em (4.7) pode ser traduzida como uma medida de homogeneidade dos dois grupos formados pelos indices de caselas. Quanto maior essa diferença, mais homogêneos serão os dois grupos internamente e heterogeneos entre si. Neste caso,

$$
\begin{aligned}
& \Delta\left(\hat{\pi}_{d}\right)= \\
& \quad=e\left\{\left[\ln \bar{y}_{J(d)} \sum_{J(d)} y_{k}+\ln \left(1-\bar{y}_{J(d)}\right) \sum_{J(d)}\left(m_{k}-y_{k}\right)+\right.\right.
\end{aligned}
$$


63.

$$
\begin{aligned}
& \left.+\ln \bar{y}_{L(d)} \sum_{L(d)} y_{k}+\ln \left(1-\bar{y}_{L(d)}\right)_{L(d)} \sum_{k .}\left(m_{k}-\bar{y}_{k}\right)\right]- \\
& -[y \ldots \ln \bar{y} \ldots+c m \ldots-y \ldots \ln <1-\bar{y} \ldots]\}
\end{aligned}
$$

tem distribuição assintótica qui-quadrado com um grau de 1 iberdade sob a hipótese de que as médias dos dois grupos de caselas definidos pela partição $\alpha$ são iguais.

4.6. A escolha da divisão mais promissora

A partição mais fina aceitavel das observações é aquela definida para cada casela ou tratamento formando um grupo e, a mais elementar, é aquel a que corresponde a um único grupo. Um caso intermediário e aquele em que os $K$ tratamentos estão divididos em $G$ grupos.

Supondo que ja ocorreram G-1 partiçes dicotônicas aplicadas sucessivamente ao conjunto de Indices $I=(1,2, \ldots, k)$, sejam $\Pi(1), \Pi(2), \ldots, \Pi(G)$, os $G$ subconjuntos formados de tal modo que

- $\Pi(i) \cap \Pi(j)=\theta, \forall i \not j, i, j=1,2, \ldots, G e \cdot \bigcup_{i=1}^{a} \Pi(i)=I$

Considerando que em cada subconjunto $\Pi(i), i=1, \ldots, G$, as médias das caselas säo iquais, seja $D C y ; \hat{\mu}_{1+0}{ }^{j}$ o desvio de 
64.

um modelo com $K$ caselas divididas em $G$ grupos de indices formados por $\Pi(1), \ldots, \Pi(a)$.

Usando as expressões (4.1), (4.2) e (4.6) pode-se escrever

$$
\begin{aligned}
& \left.D C y ; \hat{\sim}_{1+a}\right)=e\left\{\sum_{i=1}^{a} \sum_{k \in \Pi\langle i\rangle} \sum_{j=1}^{n_{k}} w_{k j}\left[y_{k j} \tilde{\theta}_{k j}-b\left(\tilde{\theta}_{k j}\right)\right]-\right. \\
& \left.-\sum_{i=1}^{a} \sum_{k \in \Pi(i)} \sum_{j=1}^{n_{k}} w_{k j}\left[y_{k j} \hat{\theta}_{i}-b\left(\hat{\theta}_{i}\right)\right]\right\}= \\
& =e\left\{\sum_{i=1}^{a} \sum_{k \in \Pi(i)} \sum_{j=1}^{n_{k}} w_{k j}\left[y_{k j} \tilde{\theta}_{k j}-b\left(\tilde{\theta}_{k j}\right)\right]-\right. \\
& \left.-\left[\sum_{i=1}^{a} \hat{\theta}_{i}\left[\sum_{k \in \Pi(i)} \sum_{j=1}^{n_{k}} w_{k j} y_{k j}\right]-\sum_{i=1}^{a} b\left(\hat{\theta}_{i}\right) \sum_{k \in \Pi(i)} \sum_{j=1}^{n_{k}} w_{k j}\right]\right\}
\end{aligned}
$$

onde, $\tilde{\theta}_{k j}=\theta\left(y_{k j}\right)$ e estimador de máxima verossimilhança no modelo com $n$ parâmetros $e \hat{\theta}_{i}=\theta\left(\hat{\mu}_{i}\right), i=1,2, \ldots, G$, é o estimador de máxima verossimilhança no modelo de $G$ grupos. O número de graus de 1 iberdade associado a (4.39) $e n-G$, com $n=\sum_{k=1}^{K} n_{k}$.

De modo análogo, o desvio do mesmo modelo considerando todas as $G$ médias de grupos iguais entre si, $D\left(y ; \hat{\mu}_{1}\right)$, tem $n-1$ graus de liberdade e pode ser expresso por

$$
D\left(y ; \hat{\mu}_{i}\right)=2\left\{\sum _ { i = 1 } ^ { a } \sum _ { k \in \Pi ( i ) } \sum _ { j = 1 } ^ { n _ { k } } w _ { k j } \left[y_{k j} \tilde{\theta}_{k j}-b\left(\tilde{\theta}_{k j} j\right]-\right.\right.
$$


65.

$$
\left.-\left[\hat{\theta} \sum_{i=1}^{o} \sum_{k \in \Pi(i)} \sum_{j=1}^{n_{k}} w_{k j} y_{k j}-\dot{b}(\hat{\theta}) \sum_{i=1}^{G} \sum_{k \in \Pi(i)} \sum_{j=1}^{n_{k}} w_{k j}\right]\right\}
$$

(4. 40$)$

ande, $\hat{\theta}=\theta\left(\hat{\mu}_{1}\right)$.

Mostra-se, facilmente, que o desvio do modelo de $G$ grupos varia entre o desvio do modelo que corresponde à partiçăo mais fina com $K$ grupos, $e$ o desvio do modelo que corresponde à partição mais rudimentar com um único grupo.

Supondo, sem perda de generalidade, que a próxima divisão ocorre no G-ésimo grupo, o conjunto de indices $I$ está partido em $G+1$ subconjuntos disjuntos formados de tal modo que

- $\Pi(i) \cap \Pi(j)=\emptyset, \forall\left(\not x_{j}, i, j=1, \ldots, G-1, G_{1}, G_{2} e\right.$

- $\left[\bigcup_{i=1}^{a-1} \cup(i)\right] \cup\left[\Pi\left(G_{1}\right)\right] \cup\left[\Pi\left(\sigma_{2}\right)\right]=I$

Considerando que em cada subconjunto $\Pi(i)$, $i=1, \ldots, G-1, G_{1}, G_{2}$, as médias das caselas são i guais, seja $D\left(y ; \hat{u}_{1+(a+1)}\right)^{2}$ desvio de um modelo com $K$ caselas divididas em $(G+1)$ grupos de indices formados por $\Pi(1), \ldots, \Pi(G-1), \Pi\left(G_{1}\right), \Pi\left(G_{2}\right)$, que pode ser reescrito por

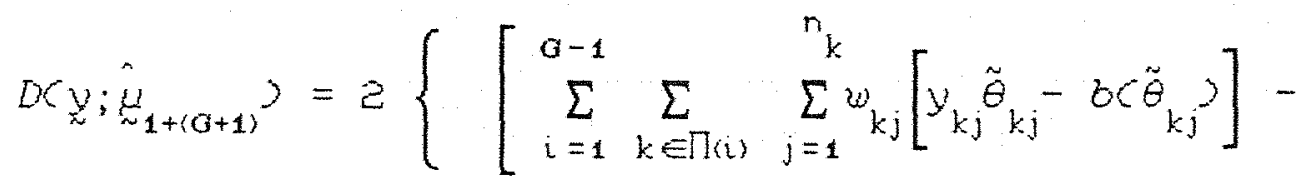


60.

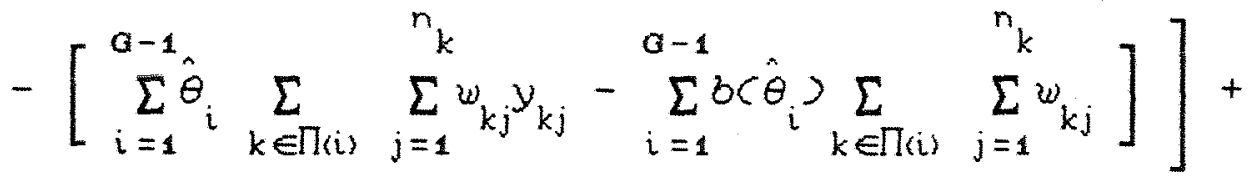

$$
\begin{aligned}
& +\left[\sum_{l} \sum_{k \in \Pi(l)} \sum_{j=1}^{n_{k}} w_{k j}\left[y_{k j} \tilde{\theta}_{k j}-b\left(\tilde{\theta}_{k j}\right)\right]-\right. \\
& \left.\left.-\left[\sum_{l} \hat{\theta}_{l} \sum_{k \in \Pi(l)} \sum_{j=1}^{n_{k}} w_{k j} y_{k j}-\sum_{l} b\left(\hat{\theta}_{l}\right) \sum_{k \in \Pi(l)} \sum_{j=1}^{n_{k}} w_{k j}\right]\right]\right\}
\end{aligned}
$$

onde,

- $\sum_{l}$ e a forma abreviada para $\sum_{l \in\left(a_{1}, a_{2}\right)}$,

$\tilde{\theta}_{k j}=\theta\left(y_{k j}\right)$,

- $\hat{\theta}_{i}=\theta\left(\hat{\mu}_{i}\right), \quad i=1, \ldots, G-1$

- $\hat{\theta}_{1}=\theta\left(\hat{\mu}_{1}\right), l=G_{1}, G_{2}$.

O número de graus de liberdade associado a (4.42) é $n-(6+1)$.

De modo análogo, o desvio do mesmo modelo considerando todas as $(G+1)$ médias de dois grupos iguais entre si, $D\left(y ; \hat{\mu}_{1}\right)$ em (4.40) pode ser reescrito por

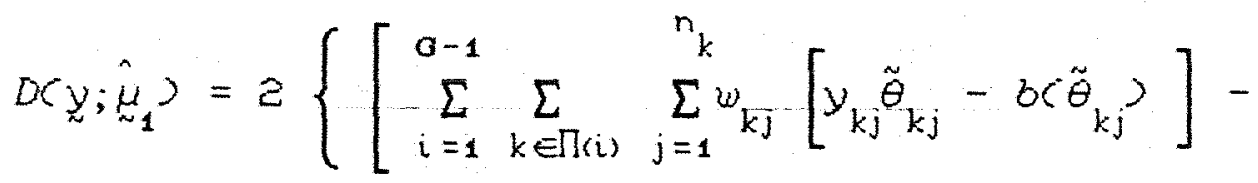


67.

$$
\begin{aligned}
& \left.-\left[\hat{\theta} \sum_{i=1}^{a-1} \sum_{k \in \Pi(i)} \sum_{j=1}^{n_{k}} w_{k j} y_{k j}-b(\hat{\theta}) \sum_{i=1}^{o-1} \sum_{k \in \Pi(i)} \sum_{j=1}^{n_{k}} w_{k j}\right]\right]+ \\
& +\left[\sum_{l} \sum_{k \in \Pi l l} \sum_{j=1}^{n_{k}} w_{k j}\left[y_{k j} \tilde{\theta}_{k j}-b C \tilde{\theta}_{k j}\right)\right]- \\
& \left.\left.-\left[\hat{\theta} \sum_{l} \sum_{k \in \Pi(l)} \sum_{j=1}^{n_{k} w_{k j}} y_{k j}-b(\hat{\theta}) \sum_{l} \sum_{k \in \Pi(l)} \sum_{j=1}^{n_{k} w_{k j}}\right]\right]\right\}
\end{aligned}
$$

onde, $\hat{\theta}=\theta\left(\hat{\mu}_{\sim_{1}}\right)$.

A diferença dos desvios (4.39) e (4.42) pode ser expressa por

$\Delta\left(\hat{\mu}_{a+1}\right)=$

$$
\begin{aligned}
& \left.=D\left(y ; \hat{\mu}_{1+a}\right)-D C y ; \hat{\mu}_{1+(\alpha+1)}\right\rangle= \\
& =e\left\{\left[\sum_{l} \hat{\theta}_{l} \sum_{k \in \Pi(l)} \sum_{j=1}^{n_{k}} w_{k j} y_{k j}-\sum_{l} b\left(\hat{\theta}_{l}\right) \sum_{k \in \Pi(l)} \sum_{j=1}^{n_{k}} w_{k j}\right]-\right. \\
& -\left[\hat{\theta}_{\sigma} \sum_{k \in \Pi(\sigma)} \sum_{j=1}^{n_{k}} w_{k j} y_{k j}-\partial\left\langle\hat{\theta}_{\sigma} \sum_{k \in \Pi(\sigma)} \sum_{j=1}^{n_{k}} w_{k j}\right]\right\}
\end{aligned}
$$

onde,

- $\hat{\theta}_{l}=\theta\left(\hat{\mu}_{l}\right), \operatorname{para} l=\sigma_{1}, G_{2} e$

- $\hat{\theta}_{a}=\theta\left(\hat{\mu}_{a}\right)$. 
A expressão $(4.44)$ útil no cálculo da proporção explicada do desvio na aplicação do método de agr upamento hierárquico divisivo e representa a contribuição que a G-ésima divisão pode dar para o cálculo de $R^{2}(G+1)$.

Supondo que o método de agrupamento divisivo hierárquico foi aplicado obtendo-se uma partição formada por $G$ grupos de $I$ de tal modo que

$$
I=\bigcup_{g=1}^{G} I_{g}
$$

cada partição d de $\Psi\left(I_{g}, \gamma\right)$ tem associada a redução $\Delta\left(\hat{\mu}_{d}\right)$ que é obtida, por exemplo, pela expressão (4.44). Seja $B\left(I_{g}, \gamma\right)$ o máximo entre as reduções $\Delta\left(\hat{\mu}_{d}\right)$, isto é

$$
\left.B\left(I_{g}, \gamma\right)=\max \left\{\Delta\left(\hat{\mu}_{d}\right): d \in \Psi I_{g}, \gamma\right)\right\}
$$

A partição do tal que

$$
\Delta\left(\hat{\mu}_{d_{0}}\right)=B\left(I_{g}, \gamma\right)
$$

pode ser considerada como a divisão dicotómica mais promissora.

Nos próximos capitulos a estatística $\Delta C \hat{\sim}_{\mathrm{d}}>\operatorname{estudada}$ quanto a sua distribuição, uma vez que sua significancia pode ser considerada como uma das regras de parada no algoritmo de divisăo dicotómica 
69.

hierárquica. 
70.

5. UMA DEFINIÇÃO MAIS GERAL DAS ESTATISTICAS E UMA ADAPTAÇÃO AO ALGORI TMO DE AGRUPAMENTO DIVISIVO HI ERARQUI CO.

5.1. Introdução

A partir da medida de homogeneidade de grupos $\Delta\left(\hat{\mu}_{G+1}\right)$, definida em $(4.44)$, é possivel generalizar a estatistica $R^{2}$, que no caso de distribuição normal é conhecida como coeficiente de determinação. Essas duas formas são utilizadas na adaptação do al gor i tmo de agr upamento divisivo hierárquico. O teste da razão de máxima verossimilhança também é deduzido como uma extensão do teste de SCOTT \& KNOTT (1974) para um modelo linear generalizado.

5.2. A estatistica $R^{2}$ generalizada

A medida da informação contida no modelo pode ser expressa pela estatistica $R^{2}$ generalizada, que corresponde à proporção do desvio explicada pelo modelo de $G$ grupos em relação ao modelo mais rudi mentar.

Utilizando as expressóes $(4.39)$ e $(4.40)$ a prorpoção do desvio pode ser obtida por 


$$
R^{2}(G)=1-D\left(y ; \hat{\mu}_{1+a}\right) / D\left(y ; \hat{\mu}_{1}\right)
$$

Demonstra-se que

$$
0=R^{2}(1) \leq R^{2}(G) \leq R^{2}(K) \leq 1
$$

Outra forma reduzida no intervalo $[0,1]$ é dada por

$$
R_{*}^{2}(G)=\frac{D\left(y ; \hat{\mu}_{1}\right)-D\left(y ; \hat{\mu}_{1+O}\right)}{D\left(y ; \hat{\mu}_{1}\right)-D\left(y ; \hat{\mu}_{1+K}\right)}=\frac{R^{2}(G)}{R^{2}(X)}
$$

que pode ser interpretada como a propor ção padronizada do desvio explicada na formação de tais modelos.

Utilizando as expressões (4.39) e (4.40),

obtêm-se

$R^{2}(G)=$

$$
\begin{aligned}
& =\left\{\left[\sum_{i=1}^{a} \hat{\theta}_{i} \sum_{k \in \Pi(i)} \sum_{j=1}^{n_{k}} w_{k j} y_{k j}-\sum_{i=1}^{a} b\left(\hat{\theta}_{i}\right) \sum_{k \in \Pi(i)} \sum_{j=1}^{n_{k}} w_{k j}\right]-\right. \\
& \left.-\left[\hat{\theta} \sum_{i=1}^{a} \sum_{k \in \Pi(i)} \sum_{j=1}^{n_{k}} w_{k j} y_{k j}-b(\hat{\theta}) \sum_{i=1}^{a} \sum_{k \in \Pi(i)} \sum_{j=1}^{n_{k}} w_{k j}\right]\right\} / \\
& /\left\{\sum_{i=1}^{a} \sum_{k \in \Pi(i)} \sum_{j=1}^{n_{k}} w_{k j}\left[y_{k j} \tilde{\theta}_{k j}-\partial C \tilde{\theta}_{k j}\right\rangle\right]-
\end{aligned}
$$




$$
\left.-\left[\begin{array}{cccccc}
\hat{\theta} \sum_{i=1}^{a} & \sum_{k \in \Pi(i)}^{n_{k}} \sum_{j=1}^{n} w_{k j} y_{k j}-b(\hat{\theta}) \sum_{i=1}^{a} \sum_{k \in \Pi(i)} \sum_{j=1}^{n_{k}} w_{k j}
\end{array}\right]\right\}
$$

e

$$
\begin{aligned}
& R_{*}^{2}(\theta)=\left\{\left[\sum_{i=1}^{a} \hat{\theta}_{i} \sum_{k \in \Pi(i)} \sum_{j=1}^{n_{k}} w_{k j} y_{k j}-\right.\right.
\end{aligned}
$$

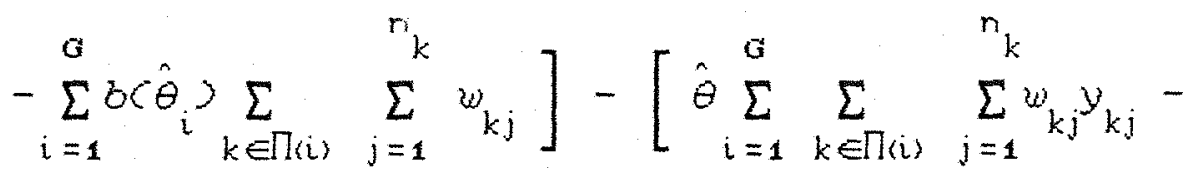

$$
\begin{aligned}
& \left.\left.-b(\hat{\theta}) \sum_{i=1}^{a} \sum_{k \in \Pi(i)} \sum_{j=1}^{n_{k}} w_{k j}\right]\right\} /\left\{\left[\sum_{k=1}^{k} \hat{\theta}_{k} \sum_{j=1}^{n_{k}} w_{k j} y_{k j}-\right.\right.
\end{aligned}
$$

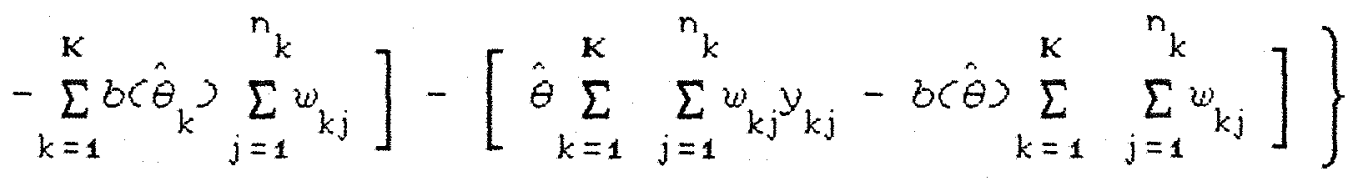

sendo que nesta última a expressão (4.2) também foi utilizada.

Supondo, sem perda de generalidade, que a próxima divisão ocorre no G-ésimo grupo, a proporção do desvio explicada na formação de um modelo de $K$ caselas divididas em $(G+1)$ grupos pode ser escrita por

$R^{2}(G+1)=$

$$
=\left\{\left[\left[\sum_{i=1}^{a-1} \hat{\theta}_{i} \sum_{k \in \Pi(i)} \sum_{j=1}^{n_{k}} w_{k j} y_{k j}-\sum_{i=1}^{a-1} \partial\left(\hat{\theta}_{i}\right) \sum_{k \in \Pi(i)} \sum_{j=1}^{n_{k}} w_{k j}\right]-\right.\right.
$$




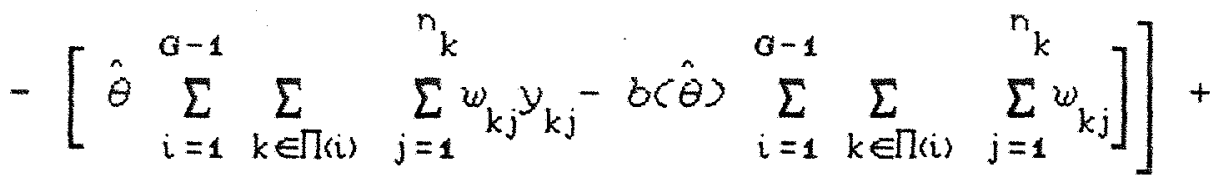

$$
\begin{aligned}
& +\left[\left[\sum_{l} \hat{\theta}_{l} \sum_{k \in \Pi(l)} \sum_{j=1}^{n_{k}} w_{k j} y_{k j}\right]-\sum_{l} \delta\left(\hat{\theta}_{l}\right) \sum_{k \in \Pi(l)} \sum_{j=1}^{\sum_{k} w_{k j}}\right]- \\
& \left.\left.-\left[\hat{\theta} \sum_{l} \sum_{k \in \Pi(l)} \sum_{j=1}^{n_{k}} w_{k j} y_{k j}-b(\hat{\theta}) \sum_{l} \sum_{k \in \Pi(l)} \sum_{j=1}^{n_{k} w_{k j}}\right]\right]\right\} / \\
& /\left\{\left[\sum_{i=1}^{a-1} \sum_{k \in \Pi(i)} \sum_{j=1}^{n} w_{k j}\left[y_{k j} \tilde{\theta}_{k j}-b<\tilde{\theta}_{k j}\right)\right]-\right.
\end{aligned}
$$

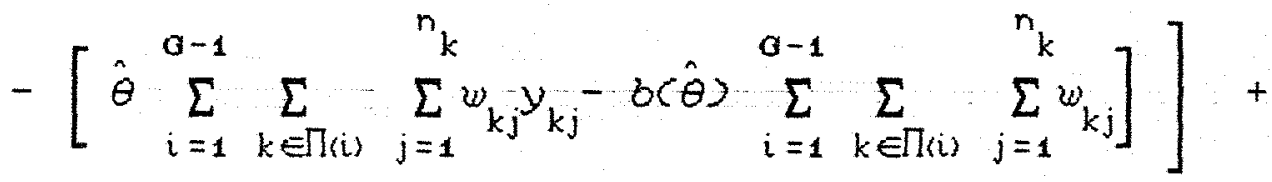

$$
\begin{aligned}
& +\left[\sum_{l} \sum_{k \in \Pi(l)} \sum_{j=1}^{n_{k}} w_{k j}\left[y_{k j} \tilde{\theta}_{k j}-\delta C \tilde{\theta}_{k j}\right]-\right. \\
& \left.\left.-\left[\hat{\theta} \sum_{i} \sum_{k \in \Pi(l)} \sum_{j=1}^{n_{k}} w_{k j} y_{k j}-b(\hat{\theta}) \sum_{l} \sum_{k \in \Pi(l)} \sum_{j=1}^{n_{k} w_{k j}}\right]\right]\right\}
\end{aligned}
$$

quando se utilizam as expressós (4.42) e (4.43).

Comparando as expressós $(5.4)$ e $(5.6)$, de $R^{2}(G)$ e $R^{2}(G+1)$, respectivamente, percebe-se que o denominador tem a mesma forma, e o numerador difere apenas na notação da $(G+1)$-esima partição do grupo $G$, para a notação da G-ésima partição que dividiu o grupo $G$ em $G_{1}$ e $G_{2}$. $O$ denominador da diferença entre as proporções explicadas do desvio na $(G+1)$-ésima partição e na G-ésima partição corresponde à diferença $\Delta\left(\hat{\mu} \hat{a}_{+1}\right)$, 
definida em (4.44).

Logo para se calcular $R^{2}(G+1)$,

recursivamente, basta ter o valor de $R^{2}(G)$ e $\Delta\left(\mu_{\sim_{G+1}}\right)$, onde este último corresponde à medida de homogeneidade entre dois grupos, $G_{1}$ e $G_{2}$, a partir do grupo ancestral $G$. Desse modo, pode-se redefinir (5.6) como

$$
R^{2}(G+1)=R^{2}(G)+\Delta\left(\hat{\sim}_{G+1}\right) / D\left(y ; \hat{\sim}_{\sim 1}\right)
$$

Para otimizar a formação de grupos homogêneos pode-se encontrar a partição $\alpha_{o}$ tal que $\Delta\left(\hat{\mu}_{\mathrm{d}}\right)$ seja máxima, para um número fixado $G$, ou então encontrar a partição de caselas tal que tenha o menor número de grupos $G$ para um nível fixado $D\left(y ; \hat{\mu}_{1+a}\right)$.

Tanto na primeira como na segunda situação a busca da partição ótima será feita através da aplicação de método de agrupamento di visivo hierárquico.

5.2.1. O caso normal

Quarido o parâmetro de dispersão é $\phi=\sigma^{2}$, $b(\theta)=\theta^{2} / 2$, a expressão (3.11) torna-se a funçãc densidade da distribuição normal... Dessa maneira, a proporção do desvio explicada na formação de um modelo de um ensaio completamente ao acaso com $k$ caselas $e n_{k}$ observações por casela, agrupadas em $G$ grupos dada em (5. 4), torna-se

$$
R^{2}(G)=\left[\sum_{i=1}^{a} n_{i} \bar{y}_{i}^{2}-n \bar{y}^{2}\right] /\left[\sum_{i=1}^{a} \sum_{k \in \Pi(i)} \sum_{j=1}^{n_{k}} y_{k j}^{2}-n \bar{y}^{2}\right]
$$


onde,

$\cdot \hat{\theta}_{i}=\theta\left(\hat{\mu}_{i}\right)=\sum_{k \in \Pi(i)} \sum_{j=1}^{n_{k}} y_{k j} / n_{i}=\bar{y}_{i}, i=1,2, \ldots, G$,

- $n_{i}=\sum_{k \in \prod_{(i)}} n_{k}$,

- a função de ligação é a canônica e

$w_{k j}=1$

O numer ador da expressão (5.8) corresponde a soma de quadrados de um modelo de $K$ caselas que possui $G$ médias distintas. O denominador de (5.8) corresponde à soma de quadrados total. Assim a expressão (5.8) equivale ao coeficiente de determinação, em geral indicado por $r^{2}$ (HOFFMANN \& VIEIRA, 1987), de um modelo de regressão formado por $K$ médias onde $G$ delas são distintas.

Quanto a proporção do desvio explicada padronizada, (5.5), tem-se

$$
R_{*}^{2}(G)=\left[\sum_{i=1}^{a} n_{i} \bar{y}_{i}^{2}-n \bar{y}^{-2}\right] /\left[\sum_{k=1}^{k} n_{k} \bar{y}_{k}^{2}-n \bar{y}^{2}\right]
$$

que corresponde à razão entre a soma de quadrados entre grupos e a soma de quadrados entre caselas.

Para cada nova divisão, a expressão (4.44), no caso da distribuição normal, é calculada por

$$
\Delta\left(\hat{\mu}_{\mathrm{G}+1}\right)=n_{G_{1}} \bar{y}_{a_{1}}^{2}+n_{a_{2}} \bar{y}_{a_{2}}^{2}-n_{G} \bar{y}_{a}^{2}
$$


onde,

$\cdot \bar{y}_{l}=\sum_{k \in \Pi(l)} \sum_{j=1}^{n_{k}} y_{k j} / n_{l} e$

- $n_{l}=\sum_{k \in \Pi(l)} n_{k}$, para $l=G, G_{1}, G_{2}$.

Comparando as expressões (2.21), (4.11) e (5.10) pode-se dizer que elas representam a soma de quadrados entre dois grupos em relação ao seu ancestral e servem para calcular o novo valor da estatistica $R^{2}$ após essa nova divisão. Desse modo a expressão (5.7) torna-se

$$
\begin{aligned}
R^{2}(G+1)= & R^{2}(G)+\left[n_{G_{1}} \bar{y}_{G_{1}}^{2}+n_{G_{2}} \bar{y}_{G_{2}}-n_{G} \bar{y}_{G}^{2}\right] / \\
& /\left[\sum_{k=1}^{K} \sum_{j=1}^{n_{k}} y_{k j}^{2}-n y^{-2}\right]
\end{aligned}
$$

5.2.e. O caso Poisson

Quando a variável aleatória tem distribuição de Poisson a proporção do desvio explicada na formação de um modelo de um ensaio completamente ao acaso com $K$ caselas, $n_{k}$ observaçeses por casela, agrupadas em $G$ grupos, dado em (5.4), torna-se, considerando a ligação logarítmica que é a natural, e $w_{k j}=1$, 


$$
\begin{aligned}
& R^{2}(\theta)=\left[\sum_{i=1}^{a} n_{i} \bar{y}_{i} \ln \bar{y}_{i}-n \bar{y} \ln \bar{y}\right] / \\
& /\left[\sum_{i=1}^{a} \sum_{k \in \Pi(i)} \sum_{j=1}^{n_{k}} y_{k j} \ln y_{k j}-n \bar{y} \ln \bar{y}\right]
\end{aligned}
$$

onde, $\hat{\theta}_{i}=\theta\left(\hat{\mu}_{i}\right)=\ln \bar{y}_{i}, \quad i=1, \ldots, \sigma$.

$$
\text { O numerador da expressão }
$$

corresponde à diferença de desvios de um modelo completamente ao acaso quando todas as médias são consideradas iguais entre si e quando as $k$ caselas possuem $G$ médias distintas.

Quanto à proporção explicada do desvio padronizada (5.5), obtém-se

$$
\begin{aligned}
R_{*}^{2}(\theta) & =\left[\sum_{i=1}^{a} n_{i} \bar{y}_{i} \ln \bar{y}_{i}-n \bar{y} \ln \bar{y}\right] / \\
/ & {\left[\sum_{k=1}^{k} \sum_{j=1}^{n_{k} n_{k} \bar{y}_{k}} \ln \bar{y}_{k}-n \bar{y} \ln \bar{y}\right] }
\end{aligned}
$$

que corresponde à razão entre a diferença de desvios do modelo com $G$ médias e uma média, e a diferença de desvios do modelo com $K$ médias e uma média.

Para cada divisão a expressão $(4.44)$ e calculada. No caso Poisson, ela é da forma

$$
\begin{aligned}
& \Delta\left(\hat{\mu}_{a+1}\right)= \\
& =2\left[n_{a_{1}} \bar{y}_{a} \ln \bar{y}_{a_{1}}+n_{a_{2}} \bar{y}_{a} \ln \bar{y}_{a_{2}}-n_{a} \bar{y}_{a} \ln \bar{y}_{a}\right]
\end{aligned}
$$


onde,

$\cdot \bar{y}_{l}=\sum_{k \in \Pi(l)} \sum_{j=1}^{n_{k}} y_{k j} / n_{l} e$

- $n_{l}=\sum_{k \in \Pi(l)} n_{k}$, para $l=G, G_{1}, G_{2}$.

Comparando (4.16) com (5.14), pode-se dizer que elas representam a diferença de desvios entre dois grupos em relação ao seu ancestral, e a expressão (5.7) pode ser obtida por

$$
\begin{aligned}
& R^{2}(G+1)=R^{2}(G)+\left[n_{a_{1}} \bar{y}_{a_{1}} \ln \bar{y}_{a_{1}}+n_{a_{2}} \bar{y}_{a_{2}} \ln \bar{y}_{a_{2}}-\right. \\
& \left.-n_{a} \bar{y}_{G} \ln \bar{y}_{G}\right] /\left[\sum_{k=1}^{k} \sum_{j=1}^{n_{k}} y_{k j}-n \bar{y} \ln \bar{y}\right]
\end{aligned}
$$

uma vez que (5.14) já foi calculada quando da divisão do conjunto de indices $G$ em $G_{1}$ e $G_{2}$.

5. 2. 3. O caso binomial

A função desvio ( 3.6 ) é utilizada quando a variável de interesse tem distribuição binomial, tendo, porém, a mesma notação do desvio.

Sendo um ensaio completamente ao acaso com $K$ caselas e $n_{k}$ observaçós por casela, sob a hipótese de 
que o conjunto de 1 ndices de caselas, $I$, está subdividido em $G$ subconjuntos disjuntos, $\Pi(i), i=1,2, \ldots, G$, de tal modo que sua união dá o próprio conjunto $I$, o parâmetro $\pi_{k j}$ é da forma $\pi_{k j}=\pi_{i}$ se $k \in \Pi(i), \forall i, j, k$. Logo o logaritmo da funçăo de verossimilhança é dado, então, por

$$
2\left(\pi_{1+a} ; y\right)=
$$$$
=\sum_{i=1}^{a} \sum_{k \in \Pi(i)} \sum_{j=1}^{n_{k}}\left[y_{k j} \ln \pi_{i}+\left(m_{k j}-y_{k j}\right) \ln \left(1-\pi_{i}\right)\right]
$$

que atinge seu máximo, considerando a função de ligação canónica, em

$$
\begin{aligned}
\hat{r}_{i}=\bar{y}_{i} & =\left[\sum_{k \in \Pi(i)} \sum_{j=1}^{n_{k}} y_{k j}\right] /\left[\sum_{k \in \Pi(i)} \sum_{j=1}^{n_{k}} m_{k j}\right]= \\
& =\left[\sum_{k \in \Pi(i)} y_{k}\right] /\left[\sum_{k \in \Pi(i)} m_{k}\right]=y_{i} / m_{i}
\end{aligned}
$$

e como valor predito de cada observação

$$
\hat{\mu}_{k j}=m_{k j} \bar{y}_{i} \text {, se } k \in \Pi(i)
$$

Assim a expressão (5.16) calculada no seu ponto de máximo é dada por

$2\left(\hat{\pi}_{1+a} ; y\right)=$ 
80.

$$
\begin{aligned}
& =\sum_{i=1}^{a} \sum_{k \in \Pi(i)} \sum_{j=1}^{n_{k}}\left[y_{k j} \ln \bar{y}_{i}+\left(m_{k j}-y_{k j}\right) \ln \left(1-\bar{y}_{i}\right)\right] \\
& =\sum_{i=1}^{a}\left[y_{i} \ln \bar{y}_{i}+\left(m_{i}-y_{i}\right) \ln \left(1-\bar{y}_{i}\right)\right]
\end{aligned}
$$

Utilizam-se as expressões (4.20) e (5.19) para se obter a função desvio

$$
\begin{aligned}
& D\left(\underset{x}{y} ; \hat{\pi}_{1+a}\right)= \\
& =2\left\{\sum_{k=1}^{k} \sum_{j=1}^{n_{k}}\left[y_{k j} \ln y_{k j}+c m_{k j}-y_{k j}\right) \ln \left(m_{k j}-y_{k j}\right\rangle-\right. \\
& \left.\left.-m_{k j} \ln m_{k j}\right]-\sum_{i=1}^{a}\left[y_{i} \ln \bar{y}_{i}+\left(m_{i}-y_{i}\right) \ln \left(1-\bar{y}_{i}\right)\right]\right\} \\
& \text { (5. } 20)
\end{aligned}
$$

A estatistica $R^{2}(G)$, que corresponde à proporção do desvio explicada pelo modelo de $G$ grupos em relação ao modelo mais elementar, pode ser obtida usando as expressóes (4.29) e (5.19).

$$
\begin{aligned}
R^{2}(G)= & \left\{\sum_{i=1}^{a}\left[y_{i} \ln \bar{y}_{i}+\left(m_{i}-y_{i}\right) \ln \left(1-\bar{y}_{i}\right)\right]-\right. \\
& \left.-\left[y y_{k} \ln \bar{y}+\left(m^{-y}\right) \ln (1-\bar{y})\right]\right\} / \\
& /\left\{\sum _ { k = 1 } ^ { k } \sum _ { j = i } ^ { n } \left[y_{k j} \ln y_{k j}+\left(m_{k j}-y_{k j}\right) \ln \left(m_{k j}-y_{k j}\right\rangle-\right.\right.
\end{aligned}
$$




$$
\left.-m_{k_{j}} \ln m_{k j}\right]-[y \ldots \ln \bar{y}+(m \ldots-y \ldots \ln (1-\bar{y})]\}
$$

A proporção padronizada do desvio explicada na formação de modelos de $G$ grupos pode ser obtida por

$$
\begin{aligned}
R_{*}^{2}(G)= & \left\{\sum_{i=1}^{G}\left[y_{i} \ln \bar{y}_{i}+\left(m_{i}-y_{i}\right) \ln \left(1-\bar{y}_{i}\right)\right]-\right. \\
& \left.-\left[y_{\ldots} \ln \bar{y}+\left(m \ldots y^{\prime}\right) \ln (1-\bar{y})\right]\right\} / \\
& /\left\{\sum_{k=1}^{k}\left[y_{k} \ln \bar{y}_{k}+\left(m_{k}-y_{k}\right) \ln \left(1-\bar{y}_{k}\right)\right]-\right. \\
& -[y \ldots \ln \bar{y}+(m \ldots-y) \ln (1-\bar{y})]\}
\end{aligned}
$$

Supondo, sem perda de generalidade que a G-ésima divisão ocorre no subconjunto $\Pi(a)$, então o conjunto de indices $I$ está partido em $G+1$ subconjuntos disjuntos, $\quad \Pi(i), \quad i=1, \ldots, G-1, G_{1}, G_{2}, \quad$ seguindo as expresşอง $(4.41)$.

Assim, para um modelo de $K$ caselas divididas em $G+1$ grupos, a função desvio pode ser escrita por

$D\left(y ; \hat{\pi}_{\sim 1+(a+1)}\right)=$ 


$$
\begin{aligned}
& =e\left\{l\left(\tilde{\pi}_{i} ; y\right)-\left[\sum_{i=1}^{a-1}\left[y_{i} \ln \bar{y}_{i}+\left(m_{i}-y_{i}\right) \ln \left(1-\bar{y}_{i}\right)\right]+\right.\right. \\
& \left.\left.+\sum_{l}\left[y_{a_{l}} \text { in } \bar{y}_{a_{l}}+\left(m_{a_{l}}-y_{a_{l}}\right) \ln \left(1-\bar{y}_{a_{l}}\right)\right]\right]\right\}
\end{aligned}
$$

E portanto o numerador da proporção da função desvio explicada na formação do modelo de $G+1$ grupos é da forma

$$
\begin{aligned}
& D\left(y ; \tilde{\pi}_{-1}\right)-D\left(y ; \tilde{\pi}_{1+(G+1)}\right)=e\left[2\left(\hat{\pi}_{\sim_{1+(G+1)}} ; y\right)-2\left(\hat{\pi}_{\sim_{1}} ; y\right)\right]= \\
& =2\left\{\left[\sum_{i=1}^{a-1}\left[y_{i} \ln \bar{y}_{i}+\left(m_{i}-y_{i}\right) \ln \left(1-\bar{y}_{i}\right)\right]+\right.\right. \\
& +\sum_{l}\left[y_{a_{l}} \ln \bar{y}_{a_{l}}+\left(m_{a_{l}}-y_{a_{l}}\right) \ln \left(1-\bar{y}_{a_{l}}\right)\right]- \\
& -[y . \ln \bar{y}+c m \ldots-y) \ln (1-\bar{y})]\}
\end{aligned}
$$

A expressão (5.24) quando comparada com o numerador da expressão (5.21) é quase que a mesma, a não ser a contribuição do G-ésimo grupo, nesta última, e a contribuição dos grupos descendentes $G_{1}$ e $G_{2}$, na primeira. Então para estudar a diferenca $R^{2}(\theta+1)-R^{2}(G)$, basta examinar a diferença dos numeradores, $\left.\Delta \hat{\pi}_{\mathrm{G}+1}\right)$, uma vez que os denominadores são iguais.

$$
\left.\Delta \hat{\pi}_{\sim_{G+1}}\right\rangle=2\left\{\left[y_{G_{1}} \ln \bar{y}_{G_{1}}+\left(m_{G_{1}}-y_{G_{i}}\right) \ln \left(1-\bar{y}_{G_{1}}\right)\right]+\right.
$$




$$
\begin{gathered}
+\left[y_{a_{2}} \ln \bar{y}_{a_{2}}+\left(m_{a_{2}}-y_{a_{2}}\right) \ln \left(1-\bar{y}_{a_{2}}\right)\right]- \\
\left.-\left[y_{a} \ln \bar{y}_{a}+\left(m_{a}-y_{a}\right) \ln \left(1-\bar{y}_{a}\right)\right]\right\}
\end{gathered}
$$

Com esses resultados, para calcular $R^{2}(G+1)$, basta ter o valor de $R^{2}(G)$ e $\Delta\left(\hat{\pi}_{a+1}\right)$, que corresponde à medida de homogeneidade entre dois grupos, $G_{1} \in G_{2}$, a partir do grupo ancestral $G$, de acordo com a forma (4.34). Assim (5.21) pode ser definida recursivamente por

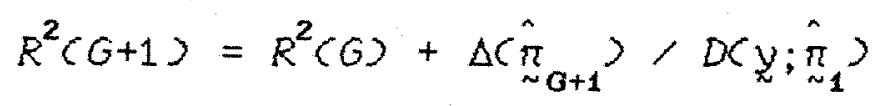

5.3. O algoritmo de agr upamento hierárquico divisivo

Conforme apresentado no Capitulo 2 , o método de agrupamento hierárquico divisivo é um procedimento simples, composto de tres passos:

1. No inicio todas as caselas estão em um único agrupamento. Por algum procedimento óti mo esse gr upo pode ser dividido em dois.

2. Outro critério de seleção escolhe um (ou os dois) dos grupos resultantes e divide-os novamente.

3. o passo anterior é repetido até que o número total de grupos final é atingido ou alguma regra de parada é satisfeita. 
No primeiro passo, o agrupamento homogéneo para uma única variável preditora, com ou sem restriçőes nas partiçōes, usado nas técnicas propostas por FISHER (1958), pode ser visto como formador do conjunto de partições donde será escolhida a partição otima.

No caso de se usar uma regra com restriçăo, o conjunto de partições dicotómicas é formado naturalmente pelas condicóes impostas. No caso de ser sem restrição nas partições dicotomicas, a busca pode se restringir ao subconjunto das partições formadas a partir da regra monotonica, que em última instancia forma os grupos dicotómicos or denando as observações de acordo com - valor medio estimado para cada casela.

Como criterio de seleção do grupo a ser formado, pode-se utilizar :

- o grupo cuja partição apresente o menor desvio, ou função desvio no caso binomial, sobre todas as partições dicotómicas analisadas, que corresponde ao máximo da estatistica $\Delta C \hat{\mu}_{\mathrm{d}}$;

- ou o grupo cuja partição apresente a estatistica $\left.\Delta \hat{\mu}_{d}\right)$ mais significativa, sobre todos os possiveis gr upos.

No que diz respeito à regra de parada, pode-se utilizar:

- um número $G$ de grupos, pré-estabelecido; 
85.

- ou uma estatistica baseada no desvio, que de alguma maneira deve ser julgada. Tanto a estatistica $R^{2}$, como a estatistica $R_{*}^{2}$ podem ser usadas, bem cono a significância do máximo da estatistica $\Delta\left(\hat{\mu}{ }_{\mathrm{d}}\right.$.

Em algumas alternativas apresentadas, o julgamento de uma estatística se faz necessário. No caso de estatisticas do tipo $R^{2}$ um ganho relativo minimo pode ser aplicado empiricamente, como na Subseçăo 2.5.2. Já para o julgamento do máximo da estatistica $\Delta\left(\hat{\mu}_{\mathrm{d}}\right)$, torna-se necessário conhecer sua distribuigão.

5. 4. Uma generalização do teste de SCOTT \& KNOTT (1974)

Usando a dedução de um teste da razão de máxima verossimilhança pretende-se estender o teste de SCOTT \& KNOTT (1974) para um modelo linear generalizado, no caso especifico do preditor linear representar a média de caselas, em um ensaio completamente ao acaso com $K$ tratamentos e $r_{k}$ observações por casela.

Todas as observaçóes pertencem ao mesmo membro da familia exponencial, cuja densidade e da forma (3.11), diferindo apenas, em termos da esperança de cada casela, $\mu_{k}$

Uma regra $\gamma \in$ definida para a obtenção da divisão dicotômica. Na análise do desvio rejeitou-se a hipótese de igualdade de médias das caselas e existem fortes evidências de que elas possam ser divididas em 
dois grupos homogeneos distintos:

Dessa maneira parece razoavel testar a hipotese de igualdade de média de caselas contra a alternativa de que o conjunto de médias assume apenas dois valores.

Assim para o conjunto de indices $I=\{1, \ldots, k\}$, qualquer partição deW $I, \gamma\}$ implicará que a média das caselas é dada por $\mu_{k}=m_{J}$ se $k \in J(\alpha)$ ou $\mu_{k}=m_{L}$ se $k \in L(d)$, no espaço de parámetros $\left(\mu_{1}, \ldots, \mu_{k}\right)$.

Seja $\left\langle\left(\hat{\mu}_{1} ; \chi\right\rangle^{\circ}\right.$ o logaritmo da função de verossimilhança maximizado sobre $\beta$ para um valor da hipótese nula tal que

$$
H_{0}: H_{k}=m, \quad k \in I \text {, }
$$

que pode ser expresso por

$$
\begin{aligned}
\left\langle\left(\hat{\mu}_{n 1} ; y\right)=\left\{\hat{\theta} \sum_{k=1}^{k} \sum_{j=1}^{n_{k}} w_{k j} y_{k j}-b(\hat{\theta}) \sum_{k=1}^{k} \sum_{j=1}^{n_{k}} w_{k j}\right\} / \phi_{0}+\right. \\
+\sum_{k=1}^{k} \sum_{j=1}^{n_{k}} c\left(y_{k j}, \phi_{o}\right)
\end{aligned}
$$

onde, $\hat{\theta}=\theta\left(\hat{u}_{i}\right)$ e estimador de máxima verossimilhança e $a_{k j}\left(\phi_{0}\right)=\phi_{0} / w_{k j}$. Considere, também, $2\left\langle\hat{\mu}_{d} ; y\right\rangle$ o logaritmo da verossimilhança maximizado sobre $\beta$ para um valor da hipótese al ternativa

$$
H_{a}: H_{k}=\left\{\begin{array}{l}
m_{J} \text { se } k \in J(\alpha) \\
m_{L} \text { se } k \in L(\alpha)
\end{array}\right.
$$


para algum $d \in \Psi(I, \gamma)$ onde $m_{J} e m_{L}$ são as médias desconhecidas de dois grupos. O logaritmo da função de verossimilhança calculado no ponto de máximo sob a hipótese al ternativa $e$ dado por

$$
\begin{aligned}
& \left.2 C \hat{\mu}_{d_{0}} ; y\right)= \\
& =\left\{\left[\hat{\theta}_{J\left(d_{0}\right)} \sum_{J\left(d_{0}\right)} \sum_{j=1}^{n_{k}} w_{k j} y_{k j}-b\left(\hat{\theta}_{J\left(d_{0}\right)} \sum_{J\left(d_{0}\right)} \sum_{j=1}^{n_{k}} w_{k j}\right]+\right.\right. \\
& \left.+\left[\hat{\theta}_{L(d,)} \sum_{L\left(d_{0}\right)} \sum_{j=1}^{n_{k}} w_{k j} y_{k j}-b\left(\hat{\theta}_{L(d)}\right) \sum_{L\left(d_{0}\right)} \sum_{j=1}^{n_{k}} w_{k j}\right]\right\} / \phi_{o}+ \\
& +\sum_{k=1}^{k} \sum_{j=1}^{n_{k}} c\left(y_{k j}, \phi_{o}\right)
\end{aligned}
$$

onde, $\hat{\theta}_{J\left(d_{0}\right)}=\theta\left(\hat{\mu}_{J\left(d_{0}\right)^{\prime}}\right)$ se $\left.k \in \mathcal{K}\left(\alpha_{0}\right) e \hat{\theta}_{L\left(d_{0}\right)}=\theta\left(\hat{\mu}_{L(d}\right)^{\prime}\right)$ se $k \in L\left(\alpha_{0}\right)$ são os estimadores de máxima verossimilhança, $d_{0}$ e o elemento de $\Psi(I, \gamma)$ que minimiza (5.30) e $\alpha_{k j}\left(\phi_{0}\right)$ $=\phi_{0} / w_{k j}$

Para se obter a estatistica do teste da razão de verossimilhança de $H_{0}$ us $H_{a}$, basta calcular a diferença entre (5.28) e (5.30), isto é

$2\left(\hat{\mu}_{\sim_{1}} ; y\right)-2\left(\hat{\mu}_{d_{0}} ; y\right)=$

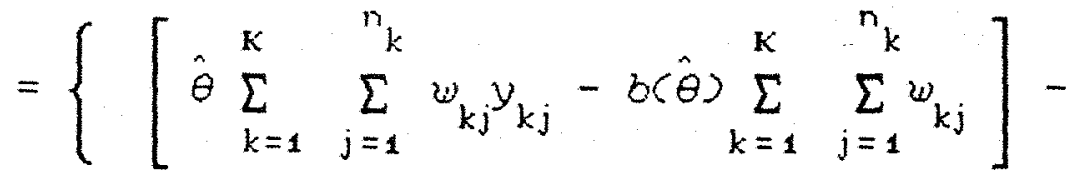




$$
\begin{aligned}
& -\left[\hat{\theta}_{J\left(d_{0}\right)} \sum_{J\left(d_{0}\right)} \sum_{j=1}^{n_{k}} w_{k j} y_{k j}-b\left(\hat{\theta}_{J\left(d_{0}\right)} \sum_{J\left(d_{0}\right)} \sum_{j=1}^{n_{k}} w_{k j}\right]-\right. \\
& -\left[\hat{\theta}_{L\left(d_{0}\right)} \sum_{L\left(d_{0}\right)} \sum_{j=1}^{n_{k}} w_{k j} y_{k j}-b\left(\hat{\theta}_{L\left(d_{0}^{\prime}\right)} \sum_{L\left(d_{0}\right)} \sum_{j=1}^{n_{k}} w_{k j}\right]\right\} / \phi_{0}
\end{aligned}
$$

Ao comparar a expressão (4.6) com a expressão (5.31), percebe-se que a primeira é proporcional à segunda. Logo, o máximo da medida de homogeneidade entre grupos, $\Delta\left(\hat{\mu}_{d}\right)$, também a estatistica usada no teste da razão de verossimil hança.

No proximo capitulo alguns resultados sobre a sua distribuição são-mostrados, quando os dados vêm de uma normal, e nos seguintes, estudos para o caso binomial são feitos por simulação. 
6. ALGUNS RESULTADOS SOBRE A DISTRIBUIÇÃO DO MĀXIMO DA ESTATISTICA $\triangle\left(\hat{\mu}_{d}\right)$ NO CASO NORMAL

6.1. Introdução.

A estatistica $B\left(I_{g}, \gamma\right)$, definida como o máximo entre as reduções $\Delta\left(\hat{\mu}_{d}\right)$ em (4.46) para um ensaio completamente ao acaso cuja variável aleatória pertença à familia exponencial, pode ser considerada como elemento relevante na escolha da divisão dicotômica mais promissora, em cada nó da árvore produzida por algoritmos de partição hierárquica dicotómica em grandes amostras.

Quando a distribuição de interesse é a normal, BUSSAB (1976) fez um estudo sobre a distribuição dessa estatística para diversas situaçōes e a seguir são mostrados alguns casos.

6. 2. O critério $\mathrm{B}$ sob a regra de divisão monotónica e mesmo número de observações por casela

Considerando que a variável aleatória é a normal, a estatística $B$, que é a redução máxima na soma de quadrados entre grupos, tem uma distribuição que é uma combinação de qui-quadrados não independentes. Tanto o número de tratamentos como o número de repetições por 
tratamento influenciam na determinação de sua distribuição.

Usando uma transformação conveniente e trabalhando com a distribuição normal multivariada, BUSSAB (1970) conseguiu obter expressões exatas para a distribuição de $B$, que no entanto, pedem a construção de tabelas apropriadas por envolverem integração numerica. Alén do mais, para propósitos mais práticos, o autor conseguiu uma aproximação conveniente para a cauda superior da distribuição usando uma aproximação da distribuição de qui-quadrado pela distribuição normal.

6.2.1. Uma transformação conveniente

Sob a hipótese de que todos os tratamentos são iguais e usando uma transformação de escala conveniente, as $k$ médias independentes, $y_{1}, y_{2}, \ldots, y_{K}$, têm a mesma distribuição normal, ou seja

$$
\underset{\sim}{\gamma^{\prime}}=\left(y_{1}, y_{2}, \ldots, y_{K}\right) \sim N\left(O_{\sim}, I\right)
$$

Sem perda de generalidade, o conjunto $I_{M}$ ordenado associado à regra $\gamma_{M}$ pode ser escrito como

$$
I_{M}=\{1,2, \ldots, K\}
$$

e também, cada partição $W_{M} I_{M} \gamma_{M}$ que é formada por dois grupos $J(d)$ e $L(d)$, onde

$$
J(d)=\{1,2, \ldots, p\} \text { e } L(d)=(p+1, p+2, \ldots, k\}
$$


Como nesse caso a partição a é determinada pelo número $\rho$, este último pode ser usado para rotulá-la e então para cada $\rho$ dado que $1 \leq \rho \leq K-1$, definem-se as estatisticas:

$$
\bar{y}=\sum_{k=1}^{k} y_{k} / k, \bar{y}(p)=\sum_{k=1}^{p} y_{k} / \rho \text { e } \bar{x}(p)=\sum_{k=p+1}^{k} y_{k} /(k-\rho)
$$

Dessa maneira a redução na soma de quadrados entre grupos como definida em (2. 21) torna-se

$$
\begin{aligned}
S Q E(\rho) & =\rho[\bar{y}(\rho)-\bar{y}]^{2}+(K-\rho)[\bar{x}(\rho)-\bar{y}]^{2} \\
& =(1 / \rho+1 /(K-\rho))^{-1}[\bar{y}(\rho)-\bar{x}(\rho)]^{2}
\end{aligned}
$$

A variável raiz quadrada de $S Q E$, denotada por 2 , é definida por

$$
\begin{aligned}
z_{p} & =[1 / \rho+1 /(k-\rho)]^{-1 / 2}[\bar{y}(\rho)-\bar{x}(\rho)] \\
& =\sum_{k=1}^{k} \alpha(k, \rho) y_{k}
\end{aligned}
$$

onde

$$
\left(2(k, p)=\left\{\begin{array}{l}
k^{-1 / 2} \varepsilon_{p} \text { se } 1 \leq k \leq p \\
k^{-1 / 2} \varepsilon_{p} \text { se } p \leq k \leq k
\end{array} \text { e } \varepsilon_{p}=[p /(K-p)]^{1 / 2}\right.\right.
$$

Indicando 0 vetor de coeficientes $\alpha(k, p)$ por $A(p)$, tem-se que 


$$
Z_{p}=A^{\prime}(\rho) Y
$$

\section{Pode-se mostrar que}

$$
\underset{\sim}{A^{\prime}}(p) \underset{\sim}{1}=0 \text { e }{\underset{\sim}{A}}^{\prime}(p) \underset{\sim}{A}(p)=1
$$

e portanto $Z_{p}$ tem distribuiçăo normal padrão e SQECp tem distribuição de qui-quadrado com um grau de liberdade. Já - vetor aleatório $\underset{\sim}{Z}$ de comprimento $k-1$ tem distribuição normal $(K-1)$-variada com vetor média nulo e matriz de correlação $R$ cujos elementos săo da forma

$$
r_{p q}=\varepsilon_{p} / \varepsilon_{q}
$$

onde $p$ e $q$ são quaisquer inteiros tais que $1 \leq p \leq q \leq K$.

Algumas vezes pode ser necessário ressaltar de qual matriz o elemento genérico provém, e então pode-se usar o simbolo $r_{\mathrm{pq}}(K)$ para indicar o elemento da matriz $R(K-1)$, bem como $g_{r}(K)$ em vez de $\varepsilon_{r}$.

Esses resultados e os de propriedade do valor máximo de estatísticas de ordem, permitem mostrar que a função distribuição acumulada da estatística $B$ é dada por

$$
\begin{aligned}
F\left(b ; I_{M}, \gamma_{M}\right) & \left.=P\left[B C I_{M}, \gamma_{M}\right) \leq b\right]= \\
& =P[\operatorname{SQEC} p) \leq b: 1 \leq p<K]= \\
& =P\left[\left|z_{p}\right| \leq \sqrt{b: 1 \leq p<K]=}\right.
\end{aligned}
$$




$$
=\int_{-\sqrt{b}}^{\sqrt{b}} \cdots \int_{-\sqrt{b}}^{\sqrt{b}} \Phi_{K-1}\left[z_{1}, \ldots, z_{k-1} \mid 0 ; R(K-1)\right] \times \prod_{p=1}^{k-1} d z p
$$

onde $\Phi_{K-1}$ é a função densidade acumulada da distribuição normal multivariada de um vetor de tamanho $k-1$ com média zero e matriz de covariância $R(K-1)$, ou seja,

$$
\begin{aligned}
& \Phi_{K-1}\{Z ; 0, R(K-1)]= \\
& =(2 \pi)^{-(K-1) / 2}|R(K-1)|^{-1 / 2} \times \exp \left[-Z_{\sim}^{\prime} R(K-1) Z_{\sim} / 2\right]
\end{aligned}
$$

Na etapa seguinte, com o objetivo de se encontrar uma expressão adequada para a redução máxi ma na soma de quadrados entre grupos, quando se utiliza a regra monotónica e se tem o mesmo número de elementos por casela, BUSSAB (1976) procurou uma transformação linear para $Z$ que de fato conduz a uma expressão mais operacional para a integral da expressão (6.10).

$$
\text { Para um } m \text { fixo, } 1 \leq m<K \text {, sejam }
$$

$$
x_{m}=z_{m} \text { e } x_{p}=\left(z_{p}-r_{p m}\right) /\left(1-r_{p m}^{2}\right)^{1 / 2}
$$

com $1 \leq \rho<K, \quad p \times m$, onde $r_{p m} \Leftrightarrow 0$ elemento de $\mathrm{R}(K-1)$ na p-ésima linha e m-esima coluna. Indicando por $c_{m}(p)$ o vetor de coeficientes com elementos não nulos apenas mas posiçóses $p$ e $m$, isto é,

$C_{m} \cdot(p)=\left(0, \ldots,\left(1-r_{p m}^{2}\right)^{-1 / 2}, 0, \ldots,-r p_{p m}\left(1-r_{p m}^{2}\right)^{-1 / 2}, \ldots, 0\right)$

e $c_{m}(m)=(0, \ldots, 1, \ldots, 0)$ 
tem-se que

$$
x_{p}=\underset{\sim m}{c_{m}}(p) Z_{\sim}
$$

o vetor $\underset{\sim}{X}$ pode ser obtido por

$$
\underset{\sim}{X}=C_{m \sim} Z
$$

considerando ${\underset{\sim}{C}}_{m}$ a matriz cujas linhas são os vetores definidos em (6.12). Quanto à sua caracterização na forma distribuicional, mostra-se que

$$
\underset{\sim}{X} N_{K-1}(\underset{\sim}{O} ; \mathrm{D}(K-1, m)
$$

onde a matriz de covariância e

$$
D(K-1, m)=C_{m} R(K-1) C_{m}
$$

e o determinante de $C_{m}$ é não nulo e igual a

$$
\left|C_{m}\right|=\prod_{\substack{p=1 \\ p \neq m}}^{k-1}\left(1-r_{p m}^{2}\right)^{-1 / 2}
$$

A estrutura da matriz de covariâncias é tal que em vez de fazer o produto das matrizes, é mais facil calcular seus elementos através das variancias e covariancias de $\underset{\sim}{X}$. BUSSAB (1976) mostrou que a matriz de covariancia definida em $(6.16)$ e da forma 
95.

$$
D(K-1, m)=\left[\begin{array}{ccc}
R(m-1) & 0 & 0 \\
0 & 1 & 0 \\
0 & 0 & R(K-m-1)
\end{array}\right]
$$

(6.18)

6.2. . A distribuição de $B_{K}$

O cálculo de probabilidade de uma variável aleatoria como (6.15) em um retangulo multidimensional, $S_{K-1}$, pode ser feito através da aplicação de integração numérica sucessivas vezes. Definindo $S_{K-1}$ como

$$
\begin{array}{r}
S_{K-1}\left(a_{k}, b_{k} ; R(K-1)\right)=P\left\{a_{k}<z_{k} \leq b_{k}, k=1, \ldots, k-1: R(K-1)\right]= \\
=\int_{a_{1}}^{b} \ldots \int_{a_{K-1}}^{b-1} \Phi_{K-1}^{b}\left[z_{1}, z_{2}, \ldots, z_{K-1}: R(K-1)\right] x_{i=1}^{k-1} \prod_{i} d z_{i} \\
\text { (6.19) }
\end{array}
$$

pode-se mostrar que, para qualquer $m$ fixado, $1 \leq m<K$,

$$
\begin{aligned}
& S_{K-1}\left(a_{k}, b_{k}: R(K-1)\right)= \\
& =\int_{a_{m}}^{b}\left\{S_{m-1}\left[a_{k}, b_{k}^{\prime}, k=1, \ldots, m-1: R(m-1)\right] \times\right. \\
& \left.\left.\times S_{K-m-1}\left[a_{j}^{\prime}, b_{j}^{\prime}, j=m+1, \ldots, K-1: R(K-m-1)\right]\right\} \times \Phi(y) d y \quad \text { (6. } 20\right)
\end{aligned}
$$

onde, 


$$
\alpha_{l}^{\prime}=\left(\alpha_{l}-r_{l m} y\right) /\left(1-r_{l m}^{2}\right)^{1 / 2} \text { e } b_{l}^{\prime}=\left(b_{l}-r_{l m} y\right) /\left(1-r_{l m}^{2}\right)^{1 / 2}
$$

Esse resultado permi te uma forma iterativa de calcular $S_{K-1}$, desde que a mesma relação pode ser aplicada novamente nos dois primeiros termos do produto do integrando. Em cada passo a dimensão do espaço retângular diminui, e o processo pára quando

$$
s_{1}(\alpha, b)=\Phi(b)-\Phi(\alpha)
$$

onde $\Phi$ e a função distribuição acumulada normal padrão uni variada.

Uma consequência imediata desses resultados é uma expressão que facilita o cálculo da função distribuição acumul ada de $B_{K}$, que é dada por

$P\left[B_{K} \leq b\right]=$

$$
\begin{aligned}
& =\int_{-\sqrt{b}}^{\sqrt{b}} S_{m-1}\left[a_{k}, b_{k}, k=1, \ldots, m: R(m-1)\right] \times \\
& \quad \times S_{K-m-1}\left[a_{j}, b_{j}, j=m+1, \ldots, k-1: R(K-m-1)\right] \times \cos (y) d y
\end{aligned}
$$

para qualquer inteiro $m$, tal que $1 \leq m<k$, onde,

$$
a_{l}=\left(-\sqrt{b}-r_{l m} y\right) /\left(1-r_{l m}^{2}\right)^{1 / 2}
$$

e

$$
b_{l}=\left(\sqrt{b}-r_{l m} y\right) /\left(1-r_{l m}^{2}\right)^{1 / 2}
$$


BUSSAB (1976) encontrou expressões mais simples se o elemento $m$ escolhido de maneira apropriada.

De posse desses conceitos, for am tabelados os valores de b satisfazendo à expressão

$$
P\left[B_{K} \leq b(\alpha, K)\right]=1-\alpha
$$

para $K$ variando de 2 até 10 e $\alpha=0,50 ; 0,25 ; 0,10 ; 0,05$; 0,01 .

6. 2. 3. Aproximação por uma distribuição qui-quadrado

Uma das maneiras de se verificar a aproximação adotada para uma distribuição é usar gráficos probabilisticos. Na presente situação BUSSAB (1976) usou - resul tado de que

$$
\left(\chi_{\nu}^{2} /(\lambda \times \nu)\right)^{1 / 3} \sim N\left(1-\frac{2}{\rho_{v}} ; \frac{2}{g_{v}}\right]
$$

mostrado por WILSON \& HILFERTY (1931), e também citado mais recentemente por DACHS (1988).

Simulando a distribuição de $B$ for am feitos gráficos dos valores de $b^{1 / 3}$ contra $\Phi^{-1}(F(b))$, obtendo-se quase linhas retas, principalmente para valores tais que F(b) são maiores que 0,50 . Logo a variável $B^{1 / 3}$ tem distribuição aproximadamente normal ou $B$ tem distribuiçăo aproximadamente $\lambda \times \chi_{\nu}{ }^{2}$. Usando, ainda, a expressão (6. 26$)$ $\lambda$ e $v$ foram obtidos das equações 
98.

$(\lambda \nu)^{1 / 3}\left(1-\frac{2}{9 \nu}\right)=\mu$ e $(\lambda \nu)^{1 / 3}\left(\frac{2}{9 \nu}\right)^{1 / 2}=\sigma$

tais que $(2 / 9 \nu)^{1 / 2}$ a raiz positiva de $x^{2}+(\mu / \gamma) x-1=0$ e $\lambda=\cos / x)^{3} / \nu$.

Uma tabela foi construída para valores de $K=3,6,10$, a partir de retas ajustadas por minimos quadrados nos pontos entre 0,50 e 0,99 produzindo equações do tipo

$$
\Phi^{-1}(F(b))=N+M b^{1 / 3}
$$

onde as correlaçós er am sempre próximas de 1,00 .

Para propósitos praticos pode-se usar a Iinha reta para calcular as probabilidades de $B_{K}$ na cauda. superior.

6. 3. O criterio $B$ sob a regra de divisão monotonica e número diferente de elementos por casela

Na situação de número de observaçóes diferente por casela, os resultados anteriormente apresentados continuam válidos, sendo que o cálculo da integral se torna mais trabalhoso.

Por essa razão o problema fol abordado por BUSSAB (1976) examinando desigualdades em vez de se calcularem os verdadeiros pontos de porcentagem da distribuição do máximo da soma de quadrados entre grupos. 
99.

médias, $y_{1}, \ldots y_{K}$, independentes, de tamanhos $n_{1}, \ldots, n_{K}$, provém de uma população normal padrăo.

$$
\underset{\sim}{y}=\left(y_{1}, \ldots, y_{K}\right) \sim N_{K}(\underset{\sim}{0} ; D)
$$

onde $\mathrm{D}$ e uma matriz diagonal, $\operatorname{com} \alpha_{k}=1 / n_{k}$.

Levando em conta o conjunto de indices associados como em (6.2), a partição a pertencente ao conjunto $K I_{M}, \gamma_{M}$ formada pelos grupos $K(\alpha)$ e $L(d)$ definidos em (6.3), e que cada partição depende somente de $\rho$ tem-se:

- os tamanhos de amostras

$$
n=\sum_{k=1}^{K} n_{k}, n(p)=\sum_{k=1}^{p} n_{k}, m(\rho)=\sum_{k=p+1}^{K} n_{k}
$$

- os totais

$$
S=\sum_{k=1}^{K} n_{k} y_{k}, S(p)=\sum_{k=1}^{p} n_{k} y_{k}, T(\rho)=\sum_{k=p+1}^{K} n_{k} y_{k}
$$

- as médias amostrais

$$
\bar{y}=s / n, \bar{y}(p)=s(p) / n(p), \bar{x}(p)=T(\rho) / m(p)(6.32)
$$

- os pesos

$$
w_{i}=n_{i} / n, i \in I_{M} ; w(p)=\sum_{i=1}^{p} w_{i} ; W=\left(w_{i}: i \in I_{M}{ }^{\prime}\right.
$$


Dessa maneira a redução na soma de quadrados entre grupos definida em (2.21) torna-se

$$
\begin{aligned}
S Q E(p) & =n(p)[\bar{y}(p)-\bar{y}]^{2}+m(p)[\bar{x}(p)-\bar{y}]^{2} \\
& =[n \times n(p) / m(p)] \times(\bar{y}(p)-\bar{y})^{2}
\end{aligned}
$$

cuja raiz quadrada é uma varíavel aleatoria normal padrão $z_{p}$, tal que

$$
z_{p}=(n \times n(p) / m(p))^{1 / 2} \times(\bar{y}(p)-\bar{y})
$$

Para cada inteiro $\rho, 1 \leq p \leq K$, pode-se também definir

$$
\varepsilon_{p}=(n(p) / m(\rho)]^{1 / 2}=[w(\rho) /(1-w(p)]]^{1 / 2}
$$

Assim usando os totais (6.31) na variável al eatoria normal padrão (6. 35 ) obtém-se a expressão

$$
z_{p}=\left(\sqrt{n} \varepsilon_{p}\right)^{-1}\left(S(p)-\varepsilon_{p}^{2} T(p)\right)
$$

6.3.1. A distribuição de $B_{K}$

Consider ando dicotomicas pertencentes a $\Psi\left(I_{M}, \gamma_{M}\right)$ a conjunta das $(K-1)$ variáveis definidas em (6.35) é dada por 


$$
\underset{\sim}{*}=\left(Z_{1}, \ldots, Z_{K-1}\right) \sim N_{K-1}(\underset{\sim}{0}, R(K-1))
$$

onde $R(K-1)$ é a matriz de correlação de elementos

$$
r_{p q}=g_{p} / g_{q}
$$

para $p$ e $q$ tais que $1 \leq p \leq q\left\langle k \in \mathcal{E}_{r}=\left(w(r) /(1-w(r))^{1 / 2}\right.\right.$.

Esses resultados mostram que a matriz $R(X-1)$ depende apenas da estrutura do conjunto $W$ e não do tamanho $n$. Pode-se, então, usar R(W) para indicar tal matriz, ou então $R(K, W)$ se for necessário o número de elementos em $W$.

Assim a distribuição acumulada de $B_{K}$, pode ser calculada por

$$
\begin{aligned}
F\left(b ; \gamma_{M}, k, W\right) & =P\left[B_{K} \leq b\right]= \\
= & \int_{-\sqrt{b}}^{\sqrt{b}} \cdots \int_{-\sqrt{b}}^{\sqrt{b}} \Phi\left(z_{1}, \ldots, z_{K-1} ; R(W) \prod_{i=1}^{K-1} d z_{k}\right.
\end{aligned}
$$

onde a matriz $R(W)$ tem elementos da forma (6.39) conforme demostrado em BUSSAB (1976).

A utilizaç̃o de tabelas abrange uma variedade de pesos e sua construção implica num processo iterativo para calcular a integral múltipla de (6. 40 ) que exige um grande esforço computacional. Esse problema foi contornado quando BUSSAB (1976) utilizou desigualdades que calculam de uma maneira aproximada os pontos de porcentagem de $B_{K}$ para algumas combinaçóes diferentes de pesos. 
6.3.2. Limites inferiores para a distribuifão de $B_{K}$ usando desigual dades

Considerando que $\left\{A_{\mathrm{p}}: \rho=1, \ldots, K-1\right\}$ um conjunto de eventos, a desigualdade de Bonferroni, que é uma expressão para a probabilidades de eventos, é dada por

$$
P\left[\begin{array}{cc}
K-1 \\
U
\end{array} A_{p=1}\right] \geq 1-\sum_{p=1}^{K-1} P\left(A_{p}^{c}\right)
$$

onde $A_{\mathrm{p}}^{\mathrm{c}}$ é o conjunto complementar de $A_{\mathrm{p}}$.

Com esse conceito BUSSAB (1976) mostrou que a distribuição acumulada da reduçăo maxima na soma de quadrados entre grupos; quando se usa a regra monotonica de divisão dicotómica e o número de elementos por casela é diferente satisfaz à expressão

$$
P\left[B_{K} \leq b\right] \geq 1-2(K-1)(1-1(\sqrt{b})]
$$

ou então, igualando o lado direito da desigualdade a 1 -a, obtém-se

$$
\left.b=\left[\Phi^{-1}(1-\alpha / 2(K-1)]\right)\right]^{2}
$$

Outro resultado obtido para a distribuiçăo acumulada usa uma das desigualdades de Sidak. Esta se refere a um vetor multinormal com media zero e matriz de correlaça arbitrária, inscrito em um espaço retangular n-dimensional, ou seja, 


$$
P\left(\left|x_{i}\right| \leq c_{i}: 1 \leq i \leq n\right) \geq \prod_{i=1}^{n} P\left(\left|x_{i}\right| \leq c_{i}\right)
$$

A variável aleatória $B_{K}$ satisfaz, então, a

$$
F\left[B_{K} \leq \delta\right] \leq[2 \Phi(\sqrt{\delta})-1]^{K-1}
$$

Para comparar as desigualdades de Bonferroni (6.42) e a de Sidak (6.45) foi usado o fato de que para qualquer inteiro positivo $n$ e para $x \leq 1$, tem-se

$$
(1-x)^{n} \geq 1-n x
$$

e para qualquer valor de $b$ tal que $\Phi(\sqrt{b}) \geq 0,50$ tem-se

$$
\Phi(\sqrt{b}) \geq 0,5 \Rightarrow 1-\Phi(\sqrt{b}) \leq 0,5 \Rightarrow \geq[1-\Phi \sqrt{b})] \leq 1
$$

Assim rearranjando a expressão (6. 45) obtém-se

$P\left[B_{K} \leq b\right] \geq[2 \Phi(\sqrt{b})-1]^{K-1}=$

$$
\begin{aligned}
& =(1-[2(1-\Phi(\sqrt{b}))])^{K-1}= \\
& \geq 1-e(K-1)[1-\Phi(\sqrt{b})]
\end{aligned}
$$

ou seja, os limites inferiores baseados na desigualdade de Sidak são menores do que ou iguais aos limites inferiores baseados na desigualdade de Bonferroni. Por essa razão os resultados produzidos pela desigualdade de Sidak são melhores que os produzidos pela desigualdade de Bonferroni.

Para analisar o comportamento de (6.47), 
BUSSAB (1976) comparou o valor da desigualdade com o valor exato (ou simulado), para um nível $K$ fixado, a uma probabilidade a e para uma matriz de estrutura $R$.

Como no caso equiponderado, a matriz $R$ depende apenas de $K$, sua análise pode ser feita através da variação de a e $K$. Fixando alguns valores de $K$, a razão entre o valor aproximado e o valor exato cou simulados tende a 1 . Entretanto para valores de $\alpha$ fixados, a razão aumenta à medida que $K$ aumenta.

Para $W$ não uniforme, BUSSAB

(1976)

observou que fixado a e $k$, a razão diminui conforme $W$ apresenta valores minimos mais próximos de zero.

Como o vetor $W$ não está explicitado na desigualdade (6.45), a desigualdade empirica de Sidak, que trata do caso em que a matriz de correlação, $\mathrm{T}_{\mathrm{K}-1}(\mathrm{r})$, tem todos os elementos for a da diagonal principal iguais. também foi estudada por BUSSAB (1976). A comparação dos resultados da simulação de $(6.43)$ com valores tabelados de uma integral semelhante a (6.40) com uma matriz uniforme sugeriu a desigualdade

$$
P\left[B_{K} \leq b\right] \geq P\left[\left|Z_{i}\right| \leq \sqrt{b}, i=1, \ldots, K-1: T_{K-1}\left(r_{\min }\right]\right\}
$$

onde

$$
\begin{aligned}
& r_{\min }=\min \left\{r_{i j}, i \neq j, i, j=1, \ldots, k-1\right\} \\
& \text { Após algumas consideraçóes tem-se que } \\
& P\left[E_{K} \leq b\right] \geq P\left[\left|Z_{\mathrm{i}}\right| \leq \sqrt{b},-i \neq j+1, \ldots K-1: \mathrm{T}_{\mathrm{K}-1}\left(r_{\min }\right)\right] \\
& \geq[2 \Phi(\sqrt{b})-1]^{K-1}
\end{aligned}
$$


ou seja, o resultado (6.48), obtido da desigualdade empirica de Sidak produz limites inferiores melhores que - resultado (6.47), obtido através da desigualdade de Sidak.

No caso equiponderado, as análises dos resultados da simulação usando a desigualdade empirica com os valores exatos de $B_{K}$ mostram que, de fato, o primeiro se aproxima do segundo conforme $r_{\min }$ tende a zero e $K$ aumenta.

No caso geral, as análises de BUSSAB (1976) também corroboram com a proposição de que (6.48) produz limites inferiores melhores, principalmente para valores grandes de $r_{\min }$ e pequenos de $k$.

6.3.3. Limites para a distribuiçăo de $B_{K}$ usando a distribuição qui-quadrado

Explorando as desigualdades já mencionadas através da integração da região sobre o elipsóide

$$
E(c: K, W)=\left\{\underset{\sim}{Z}: Z_{\sim}^{\prime} R^{-1}(W) Z Z_{\sim} \leq c^{2}\right\}
$$

BUSSAB (1976) mostrou que a variável resultante tem uma distribuiçăo qui-quadrado.

Se os volumes do elipsóide e do hipercubo são iguais, a função distribuição acumulada de $B_{K}$ é limitada superiormente por

$$
P\left[B_{K} \leq b\right]=P\left[x_{K-1}{ }^{2} \leq v(W) b\right]
$$


onde

$$
\left.\nu(W)=(4 / \pi) \times\left(\Gamma^{2}(C K+1) / 2\right) /|R(W)|\right)^{1 /(K-1)}
$$

$e$

$$
|R(W)|=w_{k} \prod_{k=1}^{k-1}\left[w_{k} /(w(k)[1-w(k)])\right]
$$

sendo

$$
w_{k}=n_{k} / n, 1 \leq k \leq K \text { e } w(l)=\sum_{i=1}^{l} w_{i}, 1 \leq l \leq K-1
$$

Por outro lado, um limite inferior para a redução máxima na soma de quadrados entre grupos no caso do uso da regra de partição monotónica e caselas com número de observações diferente, e dado por

$$
P\left[B_{K} \leq b\right] \geq P\left[\chi_{K-1}^{2} \leq b\right]
$$

A partir das expressóes (6.52) e (6.55). BUSSAB ( 1976$)$ construiu o intervalo

$$
P\left[x_{K-1}{ }^{2} \leq b\right] \leq F\left[E_{K} \leq b\right] \leq F\left[x_{K-1}{ }^{2} \leq v(W)\right] \text { (6.50) }
$$

e aproximou a distribuiçăo de $B_{\mathrm{K}}$ por uma distribuicão de qui-quadrado usando o gráfico probabilistico baseado no resultado (6.26). A partir da funça distribuiça acumulada de $E$ simulada, $F(b)$, uma reta $\left.\Phi^{-1}[F(b)]=M W\right)$ $b^{1 / 3}+N(W)$ foi ajustada por minimos quadrados nos pontos onde $0.51 \leq F(b) \leq 0.99$. Mais uma vez a aproximação se mostrou adequada nos diversos casos estudados 
apresentando coeficiente de correlação igual ou superior a 0,998 .

6. 3.4. Algumas conclusões

Quando as $k$ médias dos tratamentos, $y_{k}$, têm distribuição normal com variância $1 / n_{k}$ e são independentes, a redução máxima na soma de quadrados entre grupos quando se usa a regra $\gamma$ monotónica, $B_{K}$, pode ser aproximada por diversas maneiras, uma vez que seu valor exato é pouco operacional.

A desigualdade de Sidak, como limite superior, pode ser usada pela sua facilidade de cálculo e pela sua independencia do valor particular do conjunto de pesos. Apesar dessas vantagens, para valores grandes de $r_{\text {min }}$, definido em (6.49) o limite superior produzido é bem maior do que os valores exatos.

A desigualdade empírica, apesar de ser melhor, exige a consulta de tabelas muito especificas, e quando $r_{\text {min }}$ está próximo de zero ela está bem próxima da desi gual dade de Sidak.

Usando o resultado (6.26) a distribuiçăo de $B_{K}$ pode ser aproximada por uma distribuiça proporcional a uma qui-quadrado, que depende em grande parte do valor de $k \in r_{\text {min }}$.

Essas conelusães são válidas para valores de $K$ relativamente pequenos, pois os limites perdem sua utilidade para valores muito maiores do que 10 . 
6.4. O criterio $B$ sob a regra de divisão livre e mesmo número de observações por casela

No caso em que a regra de divisão dicotómica é a livre, as suposições iniciais a respeito das médias das caselas feitas em (6.1) podem ser utilizadas. A variável aleatória $B$ representa o máximo da soma de quadrados entre grupos sobre a classe $\psi\left(1, \gamma_{L}\right)$ de partições dicotómicas. Se $\alpha$ e uma partição qualquer definida pela regra livre, então $d$ é formada por dois subconjuntos

$J(\alpha)=\left\{j_{1}(\alpha), \ldots, j_{p}(\alpha)\right) \in L(\alpha)=\left(j_{p+1}(\alpha), \ldots, j_{K}(\alpha)\right)$

para algum $p=1,2, \ldots, k-1$. Sem perda de generalidade assume-se que a variável $B$ está sempre no conjunto principal $\mathcal{K}(d)$, ou seja, $j_{1}(d)=1$. Desse modo o número total $N(\psi)$ de partições $\alpha$ e mesmo que o número de subconjuntos principais $J(d)$. Para um número fixado $p$, constrói-se $\left(\begin{array}{l}k-1 \\ p-1\end{array}\right)$ subconjuntos diferentes $\left(1, J_{2}(d)\right.$, $J_{3}(d), \ldots, J_{p}(d)$, para $p=1,2, \ldots, k-1$, implicando que

$$
N(\psi)=\left(\begin{array}{c}
K-1 \\
0
\end{array}\right)+\left(\begin{array}{c}
K-1 \\
1
\end{array}\right)+\ldots+\left(\begin{array}{c}
K-1 \\
K-2
\end{array}\right)=2^{k-1}-1 \cdots(6.58)
$$

Cada partição $d$, com $p$ elementos no conjunto principal $J(d)$, produz

- os totais em cada subconjunto da partição 


$$
s(d)=y_{j_{1}(d)}+y_{j_{2}(d)}+\ldots+y_{j_{p}(d)}=\sum_{i \in J(d)} y_{i}
$$

e

$T(d)=y_{j_{p+1}(d)}+y_{j_{p+2}(d)}+\ldots y_{j_{k}(d)}=\sum_{i \in L(d)} y_{i}$

- as médias em cada subconjunto da partição

$\bar{y}=\sum_{i \in 1} y_{i} / k, \bar{y}(d)=s(d) / p$ e $\bar{x}(d)=T(d) /(k-p)(6.60)$

Dessa maneira a soma de quadrados entre grupos pode ser reescrita, no presente caso, como

$$
S Q E(\alpha)=[k p /(k-p)][\bar{y}(a)-\bar{y}]^{2}
$$

Sua raiz quadrada, novamente, torna-se uma transformação conveniente, da forma

$$
z_{d}=[k p /(k-p)]^{i / 2}[\bar{y}(d)-\bar{y}]
$$

Com esses elementos pode-se obter a distribuição da estatística de interesse.

6. 4.1. A distribuição de $B_{K}$

Lembrando que o objetivo é encontrar a distribuição do máximo da soma de quadrados entre grupos, quando da utilização da regra $\gamma_{L}$ sabe-se que sua funça distribuição acumulada é dada por 
$F\left(b: k, \gamma_{L}\right)=P\left[B_{K} \leq b\right]=P\left[\operatorname{SQE}(d) \leq b: d e \psi\left(I, \gamma_{L}\right)\right]$

Como cada SQECd) tem distribuição qui-quadrado com um grau de liberdade, (6.63) pode ser reescrita por

$$
P\left[B_{K} \leq b\right]=P\left[\left|z_{d}\right| \leq \sqrt{b}: d \in \psi\left(I, \gamma_{L}\right)\right]
$$

onde cada $z_{d}$ tem uma distribuição normal, uma vez que $e$ \left. uma combinação I inear de ${\underset{\sim}{*}}^{\prime}=\left(y_{1}, \ldots, y_{K}\right)^{\prime} \sim N_{\sim}, I\right)$.

o cálculo de (8.64) pode ser feito de duas maneiras. A primeira, encontrando a probabilidade do vetor

$$
Z^{\prime}=\left(z_{1}, z_{2}, \ldots, z_{N(\psi)}\right)^{\prime} \sim N_{N(\psi)}(O, \Sigma)
$$

sobre o hipercubo $\left[\underset{\sim}{Z}:\left|z_{d}\right| \leq \sqrt{b}\right]$ para todo de $k\left(I, \gamma_{L}\right)$.

A segunda meneira é encontrando a probabilidade de $Y$ sobre a região

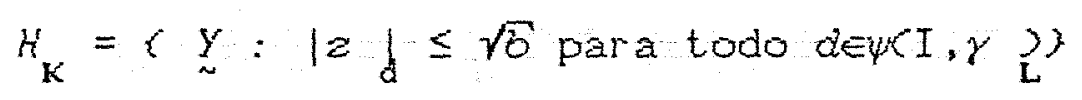

Nesta úlima, tem-se o cálculo de uma variável normal padrão $K$-variada sobre uma região bem complexa de integração. Na primeira, tem-se uma variável multinormal degenerada de alta dimensão sobre uma região bem simples.

BUSSAB (1976) optou pela segunda maneira, chegando a conclusão de que o prisma infinito $H_{K}$ possui:

- $K$ ! vertices, uma em cada uma das regiões ordenadas definidas pelos $\left({ }_{2}^{k}\right)$ hiperplanos $y_{i}=y_{j}$, ifj; 
- todos os vertices paralelos a linha $\overline{01}$;

- cada face perpendicular a um plano correspondente a $y_{i}=y_{j}, i \neq j$.

Uma simplificação para o cálculo da função distribuiçăo acumulada de $B_{K}$ quando a regra de divisão é a livre e tem-se o mesmo número de observaçóes por casel a é que por causa do efeito prisma infinito, pode-se obter a distribuição marginal no subespaço $k-1$ dimensional através da origem, perpendicular a linha $\overline{01}$. Mostra-se que a função distribuição acumulada de $B_{K}$ é

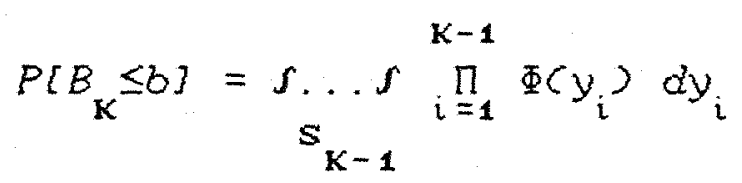

onde,

$$
S_{K-1}=H_{k} \cap(1, y=0)
$$

Apesar da região $S_{K-1}$ acima ser bem caracterizada, a integral (6.65) é muito difícil de ser achada analiticamente. Uma alternativa é se obterem desigualdades baseadas nas propriedades geometricas de $S_{K-1}$

6.4.2. Desigualdades que limitam a distribuição de $B_{K}$

Devido a sua simetria, os vértices de $S_{K-1}$, são eqüidistantes da origem. Seja o quadrado dessa distancia definido por $d^{2}(K) \times b$. Como o volume da esfera $K-1$ dimensional de raio $d^{2}(K) \times b$ é maior que o volume de $S_{K-1}$ a probabilidade de $Y$ na hiperesfera será maior que 
a probabilidade de $y$ em $S_{K-1}$.

Tem-se, então, que a função distribuição acumulada de $B_{K}$ satisfaz a

$$
P\left[B_{K} \leq b\right] \leq P\left[x_{K-1}{ }^{2} \leq d^{2}(K) \times b\right]
$$

onde, $d^{2}(K)$ e dado por

$$
d^{2}(K)=1+\sum_{p=1}^{K-2}\left[\left(1-r_{p, p+1}\right) /\left(1+r_{p, p+1}\right)\right]
$$

e

$$
r_{p, p+1}=([\rho(K-p-1)] /[(K-p)(p+1)])^{1 / 2}
$$

o estudo realizado por BUSSAB (1976) indica que esse limite é muito pobre. Por exemplo para $K=10$ e $\alpha=0,05$ o limite es $40 \%$ menor que 0 verdadeiro valor.

Por argumentos similares usados no limite superior, mostra-se que

$$
P\left[B_{K} \leq b\right] \geq P\left[\chi_{K-1}{ }^{2} \leq b\right]
$$

Tal desigualdade, entretanto, não produz também bons limites inferiores, $e$ perde sua proximidade quando $k$ aumenta, de acordo com resultados obtidos por simulação da distribuição de $B_{K}$ em BUSSAB (1976).

6.4.3. A distribuição de qui-quadrado como uma aproximação da distribuição de $B_{K}$ 
sob a regra de divisão livre e mesmo número de observações por casela, possui distribuição qui-quadrado, BUSSAB (1976) usou novamente a aproximação (6.26) e obteve bons resultados. Para propósitos práticos os pontos de porcentagem de $B_{K}$ podem ser encontrados por tal aproxi maçăo.

Os resultados de que $B_{K}$ tem distribuição qui-quadrado que $e$ limitada superiormente por uma distribuição $\chi_{\mathbf{k - 1}}^{2}$ e inferiormente, por uma constante vezes a distribuicão $\chi_{\mathbf{k - 1}}{ }^{2}$, e a especifica forma do sólido $S_{K-1}$, sugerem que deve existir uma constante $l(K)$ tal que

$$
B_{K}=\chi_{K-1}^{2} / 2(K)
$$

e que satisfaz a

$$
E\left(B_{K}\right)=(K-1) / 2(K) \text { e } \operatorname{Var}_{K}\left(B_{K}\right)=2(K-1) / l^{2}(K)(0.70)
$$

ou seja, $l(K)$ é tal que

$$
l(K)=2 \times E\left(B_{K}, \operatorname{NarC}_{K}\right)
$$

Com esses resultados BUSSAB (1976) fez simulações para diversos valores de $K e \alpha$ e construiu tabelas que servem de 1 imites superiores para os pontos de porcentagem. Esses 1 imites melhoram para valores pequenos de $k \in \alpha$, sendo este último dado por um menos a probabilidade acumulada.

Quanto aos resultados assintóticos, BUSSAB (1976), assim como SCOTT KNOTT (1974), obtiveram as 
seguintes expressões para a média e variáncia de $B_{K}$ :

$$
E\left(B_{K} / K\right) \rightarrow 2 / \pi \text { e } \operatorname{var}\left(B_{K} / \sqrt{R}\right)+8(1-2 / \pi) / \pi \quad(6.72)
$$

que diferem um pouco das expressões obtidas por HARTIGAN (1972).

Com a análise dos resultados obtidos por BUSSAB (1976) e SCOTT \& KNOTT (1974), pode-se, então dizer que a constante de proporcionalidade da distribuição de qui-quadrado definida em (6.69) converge para 1,3760 , isto é.

$$
B_{K} \sim 0,7267 \times x_{K-1}^{2}
$$

e a distribuição assintótica de $B_{K}$ é dada por

$$
(26 \pi-23 / \pi) \times x^{2}(\mathrm{~K} /(\pi-2)
$$

Esta última deve ser usada para valores de k>10. Por outro lado, para valores de $k \leq 10$ os resultados assintóticos também produzem limites inferiores melhores que os aqui apresentados anteriormente.

6.5. O critério $B$ sob a regra de divisão livre e número diferente de observações por casela

Considerando um preditor com $K$ niveis, $I=$ $\{1,2, \ldots, k\}$, a regra associada livre, $\gamma_{L}, o$ número de observações independentes na k-ésima casela como $n_{k}$, $k=1,2, \ldots K$, cada observação da variável dependente como 
sendo uma variável normal padrão, pode-se dizer que as $K$ médias de caselas $y_{1}, y_{2}, \ldots, y_{K}$ têm uma distribuição normal $K$-variada,

$$
\underset{\sim}{y} \sim N_{K}\left(0, D\left(1 / n_{1}, \ldots, 1 / n_{K}\right)\right.
$$

onde $\mathrm{D}$ é uma matriz diagonal com $\alpha_{k}=1 / n_{\mathrm{k}}$.

Cada partição dicotómica $d \in \psi\left(I, \gamma_{L}{ }^{2}\right.$, $I=\{1,2, \ldots, K\}$ é formada por dois conjuntos disjuntos da mesma forma que em (6.57). Para cada partição $d$, tem-se a correspondente redução SQE que é dada por

$$
\operatorname{SQE}(\alpha)=n^{-1}\left[[g(\alpha)]^{-1} \sum_{i \in J(d)} n_{i} y_{i}-g(\alpha) \sum_{i \in L(d)} n_{i} y_{i}\right]^{2}
$$

e a correspondente variável $\boldsymbol{z}_{d}$,

$$
z_{d}=n^{1 / 2}\left[[g(d)]^{-1} \sum_{i \in J(d)} w_{i} y_{i}-g(d) \sum_{i \in L\{d\rangle} w_{i} y_{i}\right]
$$

(6. 77$)$

onde,

$$
n=\sum_{i=1} n_{i}, \quad W=\left[w_{i}=n_{i} / n: i \in I\right], \quad w(d)=\sum_{i \in J(d)} w_{i}
$$

$e(\alpha)=(w<\alpha) /(1-w(\alpha)])^{1 / 2}$

Matricialmente tem-se que

$$
z_{d}=\underset{\sim}{A}(d) \underset{\sim}{Y}
$$


onde,

$$
A^{\prime}(\alpha)=n^{1 / 2}\left[\alpha_{1}(\alpha) w_{1}, \alpha_{2}(\alpha) w_{2}, \ldots, \alpha_{K}(\alpha) w_{K}\right]
$$

e

$$
a_{j}(\alpha)= \begin{cases}{[g(\alpha)]^{-1}} & \text { se } j \in J(\alpha) \\ -g(d) \text { se } j \in L(\alpha)\end{cases}
$$

Como anteriormente, cada SQE(d) tem distribuição qui-quadrado com um grau de liberdade $e z_{d}$ distribuição normal.

6.5.1. A função distribuição acumulada de $B_{K}$

$$
\text { O máximo da soma de quadrados entre grupos }
$$
tem como função distribuição acumul ada a expressão

$$
\begin{aligned}
& F\left(b: \gamma_{L}, W\right)=P\left[B_{k} \leq b\right]= \\
& =P\left[\operatorname{SQE}(d) \leq b: d \in \psi\left(I, \gamma_{L}\right)\right] \\
& =P\left[\left|z_{d}\right| \leq \sqrt{b}: d \in \psi\left(I, \gamma_{L}\right)\right] \\
& \text { As restriços }\left|z_{d}\right| \leq \sqrt{b} \text { geram o sólido } H_{k} \text {, } \\
& H_{K}=\left(\underset{\sim}{Y} \in \mathbb{R}^{K}:-\sqrt{b} \leq A_{\sim}^{\prime}(\alpha) Y \leq \sqrt{b}, d \in \psi\left(I, \gamma_{L}\right),(0.81)\right.
\end{aligned}
$$

Usando o fato de que

$$
Y \sim N \underset{K_{N}}{O} ; \operatorname{DC1} n_{1}, 1 / n_{2}, \ldots, 1 / n_{K}>>
$$

a função distribuição acumulada de $B_{K}$ é dada por 


$$
P\left[B_{K} \leq b\right]=P\left[Y \in H_{K}\right]
$$

Em seu estudo sobre a função acumulada do máximo da soma de quadrados entre grupos quando se utiliza a regra de divisão livre e se tem número diferente de observações por casela, BUSSAB (1976) usou uma outra expressão para a função distribuição de $B_{K}$ entretanto não possui, por exemplo, as propriedades de simetria do sólido da região de integração no caso equi ponder ado.

6.5. 2. Distribuições que limitam a distribuição de $B_{K}$

Usando o fato de que a soma de quadrados entre tratamentos é igual a soma de quadrados dentro dos grupos finais após a divisão ótima mais o máximo da soma de quadrados entre grupos, $B_{K}$, obtém-se que

$$
P\left[B_{K} \leq b\right] \geq P\left[\chi_{\mathbf{K}-1}{ }^{2} \leq b\right]
$$

Uma outra expressão que pode ser usada como limite para a função distribuição acumulada do máximo da soma de quadrados entre grupos-quando se utiliza a regra livre de divisão dicotómica com número diferente de observaçóes por casela é dada por

$$
P\left[B_{K} \leq b: \gamma_{L}, K, W\right] \leq P\left[B_{K} \leq b: \gamma_{M}, K, W\right]
$$


Este resultado foi mostrado para o caso em que $K=3$.

Percebendo que o conjunto de pesos é um fator importante, o mesmo autor fez ainda uma série de simulações usando diferentes conjuntos de $\mathrm{W}$.

Um primeiro resultado é que

$$
P\left[B_{K} \leq b: \gamma_{L}, K, W_{0}\right] \leq P\left[B_{K} \leq b: \gamma_{L}, K, W\right]
$$

onde, $W_{0}$ corresponde a amostras de mesmo tamanho, ou seja, qualquer ponto de quantil de $B_{K}$ no caso equiponderado $w_{0}$ deve ter um limite superior para o correspondente ponto de quantil em qualquer outro conjunto $w$.

Outro resultado interessante é que também nesta situação, onde o número de observaçóes por casela e diferente, a distribuição de $B_{k}$ tem um comportamento semelhante a uma variável proporcional a uma distribuição de qui-quadrado com $\nu$ graus de liberdade.

Com esses dois resultados, BUSSAB (1976) construi u tabelas da função distribuição acumulada de $B_{K}$ para a situação de regra livre de divisão dicotomica e número diferente de observações por casela.

6. 6. Resumo das aproximações.

Quando não é possivel se utilizarem as tabelas el aboradas por BUSSAB (1976), podem-se utilizar as aproximaçóes assintóticas. Assim considerando a regra de divisão monotonica, obteve os seguintes limites 
$P\left[x_{K-1}{ }^{2} \leq b\right] \leq P\left[B_{K} \leq b\right] \leq P\left[x_{K-1}{ }^{2} \leq \nu(W) b\right]$ (6.50)

onde, $\nu(W)$ é dado em (6.53).

Quando se utiliza a regra $\gamma_{L}$

$$
B_{K} \sim(2(\pi-2) / \pi) \times \chi_{(K / 4 \pi-2))}^{2}
$$

no caso equiponder ado e

$$
P\left[B_{K} \leq b\right] \geq P\left[x_{K-1}^{2} \leq b\right]
$$

quando se tem número diferente de observações por casela. Outro resul tado muito importante equando

$$
P\left[B_{K} \leq b: \gamma_{L}, K, W\right] \leq P\left[B_{K} \leq b: \gamma_{M}, K, W\right]
$$

(6. 85)

bem como

$$
P\left[B_{K} \leq b: \gamma_{L}, K, W_{O}\right] \leq P\left[B_{K} \leq b: \gamma_{L}, K, W\right]
$$

onde, $W_{0}$ corresponde a amostras de mesmo tamanho. 
7. UM ESTUDO SOBRE O COMPORTAMENTO DA DISTRIBUIÇAO DO MAXIMO DA ESTATISTICA $\Delta C \hat{\sim}_{d}$ ) NO CASO BINOMIAL

\subsection{Introdução}

A escolha da divisão mais promissora, $a_{0}$. quando da aplicação do método de agrupamento divisivo hierárquico utilizando a regra $\gamma$, entre aquel as formadas por $G$ grupos, pressupõe que a redução $\Delta\left(\hat{\mu}_{d}\right)$ seja a maior possivel. Considerando $B\left(I_{g}, y\right)$ o máxi mo entre as reduções $\Delta\left(\hat{\mu}_{d}\right)$, a partição mais promissora, $\alpha_{0}$, é aquela que satisfaz a (4.47).

No caso especifico de distribuição binomial, quando se estuda um modelo de ensaio completamente ao acaso com $n_{k}$ repetiços em cada uma das $K$ caselas, o máximo da redução $\Delta(\hat{\pi})$ definida em $(4.37)$ é utilizado para escolher a partiçăo $\alpha_{0}$ tal que

$$
\Delta\left(\hat{\pi}_{d_{0}}\right)=B\left(I_{g}, \gamma\right)=\max \left\{\Delta\left(\hat{\pi}_{d}\right): \operatorname{de\Psi }\left(I_{g}, \gamma\right)\right\}
$$

O estudo no caso da distribuição normal indica que por métodos analiticos a distribuiça da estatistica $\Delta\left(\hat{\pi}_{d_{0}}\right)$ deve ser muito complicada. Por esse motivo optou-se por seu estudo através de métodos de 
simul ação.

Como a qualidade da aproximação à distribuição binomial pela normal depende do produto $m_{k j} \times \Pi_{k}$. esses dois parâmetros foram levados em consideração além do valor do número de tratamentos envol vidos, $K$.

7.2. Descrição do programa de simulação

Usando GLIM 3.77 (PAYNE, 1986), o programa de simulação da distribuiçăo da estatistica $\Delta \hat{\pi}_{d_{0}}$ ) foi elaborado e no Apêndice 1 está uma listagem para uma das situaçōes estudadas.

Um conjunto de instruçóes de interesse que pode ser usado sempre que solicitado em um programa é chamado "macro" em GLIM. As principais "macros" que formam o programa de simulação da distribuição da estatística $\Delta \hat{\sim}_{d_{0}}$ ) são:

- MACRO SITA - Monta a tabela de dados binomiais gerados a partir da MACRO SIMULA, tendo como entrada o número de tratamentos no ensaio completamente ao acaso, o número de repetições de cada tratamento, o indice da binomial, a probabilidade da binomial,

- MACRO SIMULA - Gera uma amostra de uma distribuição binomial a partir da soma de gerações de uma uniforme $(0,1)$, onde o número de sucessos, $e$ número de vezes em que o número gerado é maior que a probabilidade da binomial, 
- MACRO SCKN - Calcula $\Delta C \hat{\pi}_{0}$, escolhida entre todas as divisões dos tratamentos, segundo a regra livre.

- MACRO ANALISE - Ajusta o modelo de análise de variáncia de um ensaio completamente ao acaso,

- MACRO CHIP - Calcula a probabilidade da cauda superior de uma distribuição de qui-quadrado usando a aproximação de WILSON \& HILFERTY (1931) à distribuicão normal, (6. 26).

As demais "macros" servem de suporte para as acima citadas.

Para a geração de diversas distribuiçós, foram escolhidos os valores 3,6 e 10 , para o número de tratamentos; 10,20 e 50, para o indice da binomial; 0,5 , $0,3 e^{0,1}$, para a probabilidade da binomial. Em cada uma das situaçós for am geradas 1000 tabelas de dados.

7. 3. A distribuição simulada de $\left.\underset{\sim \pi_{0}}{ }\right)$

Considerando a regra de divisăo livre e mesmo número de observaç̃es por casela, $n_{k}=r, \forall k$, a distribuição simulada do maximo entre as reduçes $\Delta(\hat{\pi})$ foi comparada com a distribuição qui-quadrado, uma vez que no caso normal essa estatistica tem assintoticamente esse comportamento. Por questão-de simplicidade de notaçăo, seja $\Delta_{0}$ a variavel $\Delta\left(\hat{\pi}_{d_{0}}\right)$ definida em $(7.1)$. 
Una das maneiras de se verificar a distribuicão de uma variável aleatória é através de uma análise em gráficos probabilisticos. Para a distribuição qui-quadrado utilizou-se o resultado mostrado por WILSON \& HILFERTY (1931) em (6. 26$)$ recomendada para valores da distribuifăo acumulada superiores a 0,5 .

A expressão (6. 26 ) implica que a variável $(\Delta)^{1 / 3}$ deve ter distribuiçăo aproximadamente normal ou $\Delta_{0}$ tem distribuiçöo aproximadamente $\lambda \times x_{\nu}{ }^{2}$.

Sendo $\mu$ e $\sigma^{2}$ a media $e$ a variancia de $(\Delta)^{1 / 3}$, respectivamente, os valores de $\lambda$ e $\nu$ podem ser obtidos das equaçoses (6.27).

A quantificação da análise de graficos probabilisticos se deu através do ajuste de retas de minimos quadrados nos pontos entre 0,5 e 0,99 de equaçes do tipo

$$
\Phi^{-1}\left(F\left(\Delta_{0}\right)\right)=a+b\left(\Delta_{0}\right)^{1 / 3}
$$

onde $\Phi^{-1}$ e a distribuiçăo acumulada normal padrăo e $F\left(\Delta_{0}\right)$ é a distribuiç̃o acumulada empirica da variável $\Delta_{o}$ obtida na simul ação.

Uma outra maneira de se achar o coeficiente de proporcionalidade e o número de graus de liberdade é supondo que a variavel de interesse tenha distribuicão proporcional a uma qui-quadrado, ou seja,

$$
\Delta \sim \lambda \times x_{v}^{2}
$$




$$
\left.E C \Delta_{0}\right)=\lambda \times \nu \text { e } \operatorname{var}\left(\Delta_{0}\right)=\lambda^{2} \times 2 \nu
$$

Logo

$$
\lambda=\operatorname{Var}\left(\Delta_{0}\right),\left(2 E\left(\Delta_{0}\right)\right) \text { e } \nu=E\left(\Delta_{0}\right) / \lambda
$$

No Apéndice 2 estão os resultados de uma das situações estudadas por simulação.

\subsubsection{O caso de 10 tratamentos}

Para um conjunto com 10 tratamentos e 4 repetições por tratamento foram gerados 1000 ensaios considerando $m$ igual a 50,20 e 10 e $\Pi$ igual a 0,50 , 0,30 e 0,10. Na Tabela 4 estão os resultados da simulação para a variável $\Delta_{0}^{1 / 3}$. Os coeficientes de determinação das retas ajustadas do tipo (7. Z) nunca foram inferiores a 0,994 . Isso implica que realmente a variável dada em (7.1) tem distribuição aproxi madamente qui-quadrado.

Como as medias das distribuições simuladas de $\left(\Delta_{0}\right)^{1 / 3}$ săo praticamente iguais, o mesmo ocorrendo com as variancias, para os valores estudados de $\Pi_{10} e m_{10}$ ' pode-se dizer que no caso de 10 tratamentos esses parâmetros năo produzem alteraçăo na distribuiçăo de $\left.\Delta C \hat{\pi}_{\mathrm{d}_{\mathrm{d}}}\right)$

Essa afirmação é reforçada quando se comparam os coeficientes das retas ajustadas, uma vez que são praticamente iguais.

O coeficiente de proporcionalidade $\lambda$ está bem próximo de $2(\pi-2) / n=0,7268$ e o número de graus de 
liberdade $\nu$ parece ser um pouco superior a $k(\pi-2)=$ 8,76 .

Dessa maneira parece ser conveniente usar - resultado $(6.84)$ do caso normal em que a distribuição acumulada do máximo da soma de quadrados entre grupos é limitada superiormente pela distribuiçăo qui-quadrado com $K-1$ graus de 1 iberdade.

Ao se examinar a Tabela 5 que trata da distribuição simulada de $\Delta_{0}$, esses resultados são reforçados. O parametro de proporcionalidade e o número de graus de liberdade obtidos por (7.5) são praticamente invariantes para os valores estudados de $\Pi_{\text {to }}$ e $m_{10}$. Pode-se dizer tambem que o parametro de proporcionalidade se aproxima de $2(\pi-2) / \pi$ e o número de graus de liberdade de $k /(\pi-2)$.

Na Tabela 6 tem-se a comparação entre as distribuições acumuladas de:

- ID - distribuição empírica simulada de $\Delta_{\mathrm{o}} \lambda \lambda$, onde $\lambda=2(\pi-2) / \pi$,

- QUID - distribuição acumulada de qui-quadrado com $\nu=K /(\pi-2)=8,76$ graus de 1 iberdade,

- QI - distribuiçăo acumulada de qui-quadrado com $\nu=k-1=9$ graus de 1 iberdade .

para os percentis 0,$80 ; 0,85 ; 0,90 ; 0,95 ; 0,975 ; 0,995$.

Nota-se que para valores superiores a 0,90 as aproximaçães tornam-se cada vez melhores, sendo que a aproximação representada por QUID tem desempenho mel hor 
que $Q I$.

$$
\begin{aligned}
& \text { Pode-se dizer que } \\
& \Delta_{0} \lambda \sim \chi_{\nu}{ }^{2}
\end{aligned}
$$

para $k=10$.

7.3.2. O caso de 6 tratamentos

Considerando um conjunto com 6 tratamentos e 4 repetiçöes por tratamento foram gerados 1000 ensaios de uma distribuição bi nomial com indce $m$ igual a 50, 20 e 10 e $\Pi$ igual a $0,50,0,30$ e 0,10 . Na Tabela 7 estão os resultados da simulação para a varíavel $\Delta_{0}^{-1 / 3}$. Os coeficientes de determinação das retas ajustadas do tipo (7.2) nunca foram inferiores a 0,9894 . Isso indica que para $k=6$ a variável (7.1) tem distribuição aproxi madamente qui-quadrado.

Como as médias das distribuiçães simuladas de $\left.C \Delta_{0}\right)^{1 / 3}$ são praticamente i quais, o mesmo ocor rendo com as variancias, para os valores estudados de $\Pi_{\sigma}$ e $m_{\sigma}$, pode-se dizer que no caso de 6 tratamentos esses parâmetros produzem uma pequena alteração na distribuição de $\Delta\left(\hat{\pi}_{\sim d}\right)$, quando comparado com os resultados do caso de 10 tratamentos.

Essa afirmação é reforçada quando se comparam os coeficientes das retas ajustadas, uma vez que existe uma variação maior do que no caso de 10 tratamentos. 
O coeficiente de proporcionalidade $\lambda$ está bem próximo de $2(\pi-2) / \pi=0,7268$ e o número de graus de liberdade $v$ é sempre inferior a $K /(\pi-2)=5,26$ na maioria dos casos.

Ao se examinar a Tabela 8 que trata da distribuição simulada de $\Delta_{0}$, esses resultados são novamente reforçados. O parámetro de proporcionalidade e - número de graus de liberdade obtidos por (7.5) são praticamente invariantes para os valores estudados de $\Pi_{\sigma}$ e $m_{\sigma}$. Pode-se dizer também que o parâmetro de proporcionalidade se aproxima de $2(\pi-2) / \pi$ e o número de graus de liberdade de $k /(\pi-2)$ apresenta, entretanto, uma variabilidade maior do que no caso de 10 tratamentos.

Na Tabela 9 tem-se a comparação entre as distribuições acumuladas de $I D$, QUID $e Q I$. Nota-se que para valores superiores a 0,90 de ID a aproximação torna-se cada vez melhor. A aproximação representada por QUID tem desempenho melhor que Q1. Entretanto, quando comparados com os resultados obtidos no caso de 10 tratamentos as aproximaçōes QI e QUID não tem um desempenho tão bom como no caso de 10 tratamentos.

Mesmo assim a aproximação (7.6) pode ser usada com certo cuidado para $k=6$.

7.3.3. O caso de 3 tratamentos

Para um conjunto com 3 tratamentos e 4 repetições por tratamento também for am gerados 1000 ensaios considerando $m$ igual a 50,20 e 10 e $\Pi$ igual a 
$0,50,0,30$ e 0,10 . Os resultados da simulaçăo para a variavel $\Delta_{0}^{i / 3}$ estão na Tabela 10 . Os coeficientes de determinação das retas ajustadas do tipo (7.2) nunca foram inferiores a 0,9901 . Isso implica que novamente a variavel dada em (7.1) tem distribuiçăo aproximadamente qui-quadrado. Para um conjunto com 3 tratamentos, a Tabela 13 mostra que os coeficientes de determinação das retas ajustadas do tipo (7.2) nunca foram inferiores a 0,9901 . Isso implica que novamente a variável (7.1) tem distribuição aproximadamente qui-quadrado.

Como as médias das distribuiçôes simuladas de $\left(\Delta_{0}\right)^{1 / 3}$ são também praticamente iguais, $O$ mesmo ocorrendo com as variancias, para os valores estudados de $\Pi_{3}$ e $m_{3}$, pode-se dizer que no caso de 3 tratamentos esses parâmetros produzem alguma alteração na distribuição de $\Delta C \hat{\pi}_{\mathrm{d}}$ ), quando comparados com os resultados das situações anteriores.

Essa afirmação e reforçada quando se comparam os coeficientes das retas ajustadas, uma vez que são praticamente iguais.

O coeficiente de proporcionalidade $\lambda$ e sempre superior ao valor $2(\pi-2) / \pi=0,7268$ e o número de graus de liberdade $\nu$ é sempre inferior $k /(\pi-2)=2,635$, trazendo, então, a suspeita de que, nessa situação, a aproximação já não é tão boa.

Ao se examinar a Tabela 11 que trata da distribuição simulada de $\Delta_{0}$, esses resultados são reforçados. O parâmetro de proporcionalidade e o número de graus de 1 iberdade obtidos por (7.5) são praticamente invariantes para os valores estudados de $\Pi_{3}$ e $m_{3}$. Aqui não se pode afirmar que o parametro de proporcionalidade 
se aproxima de $2(\pi-2) / \pi$ e o número de graus de liberdade de $K /(\pi-2)$ como nas situaçoes anteriores.

$\mathrm{Na}$ Tabela 12 tem-se a comparação entre as distribuições acumuladas de $I D, Q U I D$ e $Q I$. Nota-se que de fato a aproximação (7.6) não pode ser usada com segurança, pois já existe uma diferença muito grande entre a distribuição acumulada empirica e a distribuiç̃o aproxi mada.

7.4. Conclusões.

Quando a escolha de partição $\alpha_{0}$ mais promissora em uma aplicação do método de agrupamento divisivo hierárquico utilizando a regra $\gamma_{L}$, envolve a distribuição da estatistica $\Delta \hat{\sim}_{\sim_{0}}$ ' no caso de um ensaio binomial completamente ao acaso com $n_{k}$ repetições em cada uma das $K$ caselas, pode-se utilizar uma aproximação semelhante àquela usada por SCOTT \& KNOTT (1974).

A distribuição da estatística $\Delta_{0} \lambda$ pode ser aproximada por uma distribuição de qui-quadrado com $\nu$ $=K /(\pi-2)$ graus de liberdade.

Essa aproximação é recomendada para qualquer valor dos vetores $\Pi_{k} e m_{k}$ uniformes. A qualidade da aproximação depende do valor de $K, 0$ número de tratamentos: quanto maior seu valor, melhor sua apr xi mação.

Dentro dos propósitos deste trabalho, pode-se dizer que a utilização da estatistica $\Delta_{0} / \lambda$ segue os mesmos resultados obtidos por SCOTT \& KNOTT (1974) e BUSSAB (1976). 
Tabela 4 - Alguns resultados da distribuifă simulada de $\Delta_{0}^{1 / 3}$ para di versos valores de $\Pi_{10}$ e $m_{10}$ e $K=10$

\begin{tabular}{|c|c|c|c|c|c|c|c|c|}
\hline \multirow[b]{2}{*}{$\Pi_{10}$} & \multirow[b]{2}{*}{$m_{10}$} & \multirow[b]{2}{*}{ media } & \multicolumn{2}{|c|}{ Parametros } & \multicolumn{2}{|l|}{ le $\Delta_{0}^{1 / 3}$} & \multirow[b]{2}{*}{$\lambda^{2}$} & \multirow[b]{2}{*}{$v^{2}$} \\
\hline & & & variancia & $a^{1}$ & $b^{1}$ & $R^{21}$ & & \\
\hline \multirow{3}{*}{0,50} & 50 & 1,864 & 0.0843 & $-6,371$ & 3,421 & 0.9964 & 0.7245 & 9.600 \\
\hline & 20 & 1.883 & 0.0819 & -6.046 & 3,243 & 0,9981 & 0.7100 & 10,060 \\
\hline & 10 & 1,884 & 0.0840 & $-6,304$ & 3,354 & 0.9971 & 0.7295 & 9.830 \\
\hline \multirow{3}{*}{0.30} & 50 & 1.864 & 0,0825 & $-6,033$ & 3,263 & 0,9971 & 0,7090 & 9,823 \\
\hline & 20 & 1.860 & 0.0891 & -6.054 & 3,265 & 0.9945 & 0.7645 & 9,067 \\
\hline & 10 & 1.844 & 0,0907 & $-5,997$ & 3,263 & 0,9958 & 0.7722 & 8,770 \\
\hline \multirow{3}{*}{0,10} & 50 & 1.842 & 0.0857 & $-6,260$ & 3.400 & 0.9986 & 0,7279 & 9,237 \\
\hline & 20 & 1.871 & 0.0887 & $-6,352$ & 3,392 & 0.9962 & 0.7653 & 9,209 \\
\hline & 10 & 1.924 & 0,0907 & $-6,154$ & 3,214 & 0,9967 & 0,8041 & 9.510 \\
\hline
\end{tabular}

1 Valores estimados no ajuste da reta : $\operatorname{1}^{-1}\left(F(\Delta)=a+b \Delta^{1 / 3}\right.$

2 Valores estimados pelas equaçôes (6. 27 )

Tabela 5 - Alguns resultados da distribulção simulada de $\Delta_{0}$ para diversos valores de $\Pi_{10}$ e $m_{10}$ e $K=10$

\begin{tabular}{|c|c|c|c|c|c|c|}
\hline \multirow[b]{2}{*}{$\Pi_{10}$} & \multirow[b]{2}{*}{$m_{10}$} & \multicolumn{5}{|c|}{ Parametros de $\Delta_{\mathrm{o}}$} \\
\hline & & media & variancia & razao & $\lambda^{1}$ & $v^{1}$ \\
\hline \multirow{3}{*}{0.50} & 50 & 6,944 & 10,21 & 1.471 & 0.7355 & 9,441 \\
\hline & 20 & 7.147 & 10,76 & 1.506 & 0.7530 & 9.491 \\
\hline & 10 & 7.163 & 10,70 & 1,494 & 0.7470 & 9.589 \\
\hline \multirow{3}{*}{0.30} & 50 & 6.943 & 10.26 & 1,477 & 0,7385 & 9,401 \\
\hline & 20 & 6,931 & 10,93 & 1.577 & 0.7885 & 8,790 \\
\hline & 10 & 6,796 & 10,62 & 1.569 & 0,7845 & 8.663 \\
\hline \multirow{3}{*}{0.10} & 50 & 6,720 & 9,90 & 1,473 & 0,7365 & 9.124 \\
\hline & 20 & 7,052 & 10.81 & 1.533 & 0.7865 & 9.200 \\
\hline & 10 & 7.646 & 12,59 & 1.646 & 0.8230 & 9,290 \\
\hline
\end{tabular}

1 Valores estimados a partir de $\lambda=\sigma^{2} /(2 \mu)$ e $\nu=\mu / \lambda$ 
Tabela 6 - Distribuição acumulada da simulaçăo de $\Delta_{0} /(2(n-2) / n)$ (ID). distribulçăo acumul ada de uma qui-quadrado com $k /(n-2) \mathrm{g} .1$. (QUID) distribição acumulada de uma qui-quadrado com $K-1$ g.1. (OI) no caso de 10 tratamentos.

\begin{tabular}{|c|c|c|c|c|c|c|c|}
\hline \multirow[b]{3}{*}{$m_{10}$} & \multirow[b]{3}{*}{ ID } & \multicolumn{6}{|c|}{$\Pi_{10}$} \\
\hline & & \multicolumn{2}{|c|}{0,50} & \multicolumn{2}{|c|}{0.30} & \multicolumn{2}{|c|}{0.10} \\
\hline & & OI & QUID & OI & QUI D & OI & QUID \\
\hline \multirow[t]{6}{*}{50} & 0,8000 & 0.8276 & 0,8414 & 0.8287 & 0,8424 & 0,8168 & 0.8312 \\
\hline & 0,8500 & 0,8639 & 0.8755 & 0,8749 & 0.8858 & 0,8536 & 0,8659 \\
\hline & 0.9000 & 0.9168 & 0.9247 & 0,9210 & 0.9286 & 0,9091 & 0,9176 \\
\hline & 0.9500 & 0.9605 & 0.9645 & 0,9622 & 0.9663 & 0.9569 & 0,9614 \\
\hline & 0,9750 & 0,9837 & 0,9856 & 0,9847 & 0,9865 & 0,9754 & 0,9782 \\
\hline & 0,9950 & 0,9978 & 0,9981 & 0.9970 & 0,9974 & 0,9945 & 0.9952 \\
\hline \multirow[t]{6}{*}{20} & 0,8000 & 0,8441 & 0,8569 & 0.8269 & 0.8407 & 0,8422 & 0.8551 \\
\hline & 0,8500 & 0.8841 & 0.8943 & 0.8843 & 0.8946 & 0.8834 & 0.8937 \\
\hline & 0,9000 & 0.9338 & 0,9403 & 0.9266 & 0.9337 & 0,9199 & 0.9276 \\
\hline & 0,9500 & 0,9500 & 0,9716 & 0,9622 & 0,9663 & 0.9673 & 0,9709 \\
\hline & 0.9750 & 0,9681 & 0,9888 & 0,9861 & 0.9877 & 0.9863 & 0.9879 \\
\hline & 0,9950 & 0.9972 & 0.9981 & 0.9985 & 0,9988 & 0.9960 & 0,9966 \\
\hline \multirow[t]{6}{*}{10} & 0,8000 & 0.8510 & 0.8634 & 0,8235 & 0,8376 & 0.8767 & 0,8875 \\
\hline & 0,8500 & 0,8838 & 0,8941 & 0,8619 & 0,8736 & 0.9123 & 0,9205 \\
\hline & 0,9000 & 0,9323 & 0,9390 & 0,9043 & 0.9132 & 0.9553 & 0,9600 \\
\hline & 0,9500 & 0.9722 & 0,9753 & 0,9621 & 0.9662 & 0.9808 & 0,9830 \\
\hline & 0,9750 & 0,9849 & 0,9867 & 0,9776 & 0.9802 & 0,9897 & 0,9910 \\
\hline & 0,9950 & 0,9976 & 0.9979 & 0,9982 & 0.9985 & 0.9992 & 0.9993 \\
\hline
\end{tabular}


Tabela 7 - Alguns resultados da distribuiçăo simulada de $\Delta_{0}^{1 / 3}$ para diversos valores de $\Pi_{\sigma}$ e $m_{\sigma}$ e $k=6$

\begin{tabular}{|c|c|c|c|c|c|c|c|c|}
\hline \multirow[b]{2}{*}{$n_{0}$} & \multirow[b]{2}{*}{$n_{\sigma}$} & \multirow[b]{2}{*}{ media } & \multicolumn{2}{|c|}{ Parametros } & \multicolumn{2}{|c|}{ de $\Delta_{0}^{1 / 3}$} & \multirow[b]{2}{*}{$\lambda^{2}$} & \multirow[b]{2}{*}{$\nu^{2}$} \\
\hline & & & variancia & $a^{1}$ & $b^{1}$ & $R^{21}$ & & \\
\hline \multirow{3}{*}{0,50} & 50 & 1.530 & 0,1058 & $-4,664$ & 3,051 & 0,9966 & 0,7600 & 5,352 \\
\hline & 20 & 1.525 & 0,1123 & $-4,437$ & 2,914 & 0.9965 & 0.8062 & 5,037 \\
\hline & 10 & 1.538 & 0.1066 & - & - & - & 0.7515 & 5,118 \\
\hline \multirow{3}{*}{0.30} & 50 & 1.517 & 0.1065 & $-4,014$ & 2,716 & 0,9963 & 0.7592 & 5,237 \\
\hline & 20 & 1.516 & 0,1105 & $-4,632$ & 3.043 & 0.9990 & 0.7884 & 5.057 \\
\hline & 10 & - & - & - & - & - & - & - \\
\hline \multirow{3}{*}{0,10} & 50 & 1.508 & 0.1124 & -4.553 & 3.009 & 0,9894 & 0.7987 & 4,930 \\
\hline & 20 & 1.523 & 0,1119 & $-4,373$ & 2.890 & 0,9957 & 0,8023 & 5.041 \\
\hline & 10 & 1.508 & 0.1124 & - & - & - & 0.7987 & 4,930 \\
\hline
\end{tabular}

1 Valores estimados no ajuste da reta : $\Phi^{-1}(F(\Delta))=a+b \Delta^{1 / 3}$

2 Valores estimados pelas equaçzes (6. 27)

- Valores perdidos devido a problemas internos do pacote

Tabela 8 - Alguns resultados da distribuição simulada de $\Delta_{0}$ para diversos valores de $\Pi$ o $m$ o $K=6$

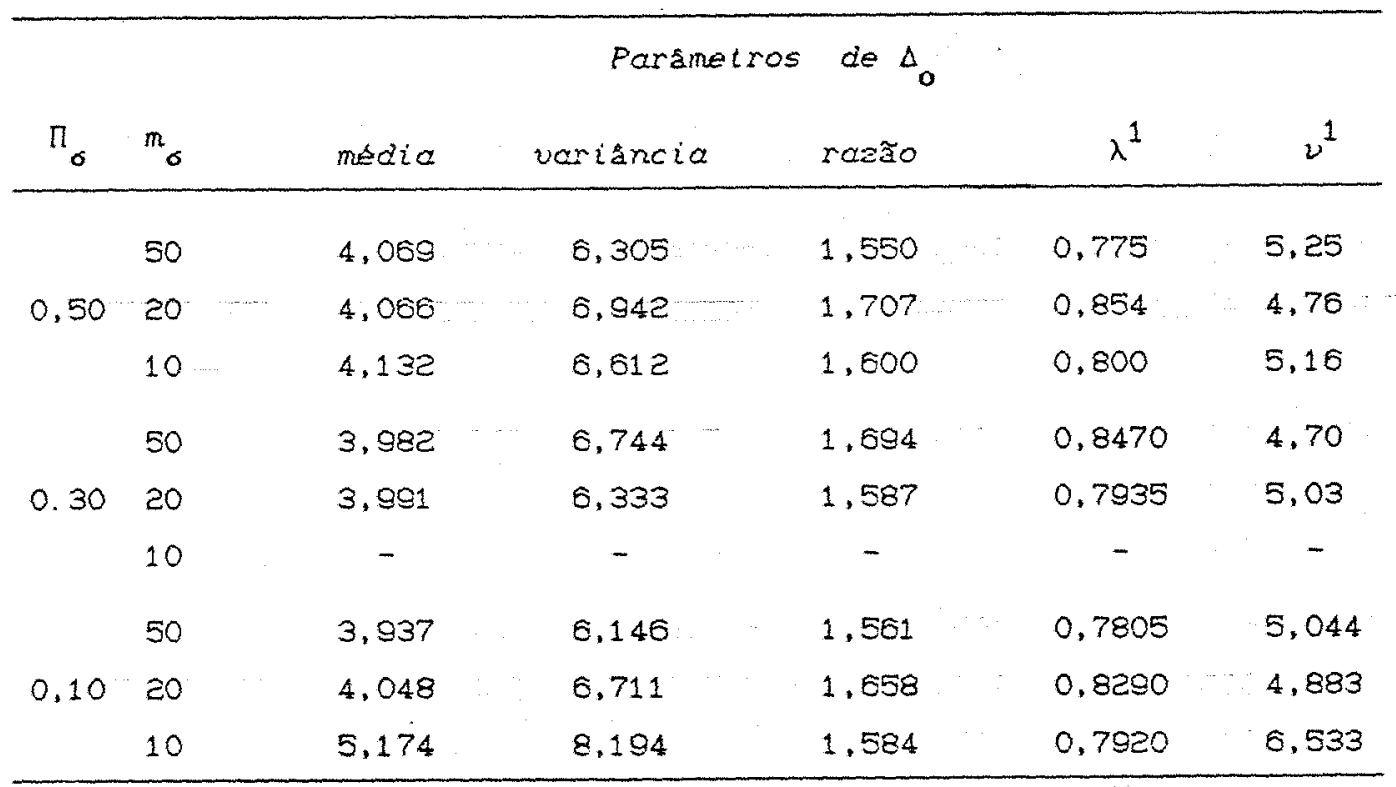

1 Valores estimados a partir de $\lambda=\sigma^{2} /(2 \mu)$ e $\nu=\mu / \lambda$

- Valores perdidos devido a problemas internos do pacote. 
Tabela 9 - Distribuição acumulada da simulação de $\Delta_{0} /(2(\pi-2) / \pi)$ (ID), distribuição acumulada de uma qui-quadrado com $k(\pi-2)$ g. 1 . (QUID) e distribição acumulada de uma qui-quadrado com $k-1$ g.1. (OI) no caso de 6 tratamentos.

\begin{tabular}{|c|c|c|c|c|c|c|c|}
\hline \multirow[b]{3}{*}{$m_{\sigma}$} & \multirow[b]{3}{*}{ ID } & \multicolumn{6}{|c|}{$\pi_{0}$} \\
\hline & & \multicolumn{2}{|c|}{0,50} & \multicolumn{2}{|c|}{0.30} & \multicolumn{2}{|c|}{0.10} \\
\hline & & QI & QUID & QI & QUID & QI & QUTD \\
\hline \multirow[t]{6}{*}{50} & 0,8000 & 0,8520 & 0,8331 & 0,8322 & 0,8116 & 0,8432 & 0,8235 \\
\hline & 0.8500 & 0.8877 & 0.8723 & 0,8866 & 0,8711 & 0,8942 & 0.8795 \\
\hline & 0,9000 & 0,9257 & 0,9145 & 0,9298 & 0,9191 & 0,9372 & 0,9275 \\
\hline & 0.9500 & 0,9686 & 0,9632 & 0,9760 & 0,9717 & 0,9720 & 0,9672 \\
\hline & 0.9750 & 0,9842 & 0,9812 & 0,9863 & 0,9837 & 0,9811 & 0.9776 \\
\hline & 0,9950 & 0,9978 & 0,9973 & 0.9987 & 0,9984 & 0,9896 & 0,9879 \\
\hline \multirow[t]{6}{*}{20} & 0.8000 & 0,8481 & 0,8289 & 0.8430 & 0.8233 & 0,8593 & 0.8411 \\
\hline & 0,8500 & 0,8978 & 0.8835 & 0,8875 & 0,8721 & 0,8996 & 0,8855 \\
\hline & 0,9000 & 0,9300 & 0,9194 & 0,9258 & 0,9147 & 0.9343 & 0,9242 \\
\hline & 0.9500 & 0,9703 & 0.9651 & 0.9653 & 0,9594 & 0.9728 & 0,9680 \\
\hline & 0,9750 & 0,9853 & 0,9825 & 0,9844 & 0.9815 & 0,9885 & 0,9863 \\
\hline & 0.9950 & 0,9983 & 0,9980 & 0,9965 & 0,9958 & 0,9971 & 0,9965 \\
\hline \multirow[t]{6}{*}{10} & 0,8000 & - & - & - & - & - & - \\
\hline & 0,8500 & - & - & - & - & - & - \\
\hline & 0.9000 & - & - & - & - & - & - \\
\hline & 0,9500 & - & - & - & - & - & - \\
\hline & 0,9750 & - & - & - & - & - & - \\
\hline & 0,9950 & - & - & - & - & - & - \\
\hline
\end{tabular}

- Valores perdidos devido a problemas internos do pacote 
Tabela 10 - Alguns resultados da distribuiça simulada de $\Delta_{0}^{1 / 3}$ para diversos valores de $n_{3}$ e $m_{3}$ e $k=3$

\begin{tabular}{|c|c|c|c|c|c|c|c|c|}
\hline \multirow[b]{2}{*}{$\Pi_{3}$} & \multirow[b]{2}{*}{$m_{3}$} & \multirow[b]{2}{*}{ média } & \multicolumn{2}{|c|}{ Parametros } & \multirow{2}{*}{ de $\begin{array}{c}\Delta_{0}^{1 / 3} \\
b^{1}\end{array}$} & \multirow[b]{2}{*}{$R^{21}$} & \multirow[b]{2}{*}{$\lambda^{2}$} & \multirow[b]{2}{*}{$v^{2}$} \\
\hline & & & variancia & $a^{1}$ & & & & \\
\hline \multirow{3}{*}{0.50} & 50 & 1,104 & 0.1551 & $-2,786$ & 2,521 & 0.9972 & 0,8585 & 2,170 \\
\hline & 20 & 1.108 & 0.1625 & -2.525 & 2,323 & 0.9939 & 0.9061 & 2,100 \\
\hline & 10 & 1.111 & 0,1478 & $-2,759$ & 2,504 & 0.9930 & 0.8199 & 2.279 \\
\hline \multirow{3}{*}{0.30} & 50 & 1.113 & 0.1601 & -2.543 & 2,330 & 0.9985 & 0.8947 & 2.141 \\
\hline & 20 & 1.116 & 0,1595 & $-2,815$ & 2,510 & 0,9970 & 0,8930 & 2,157 \\
\hline & 10 & 1.114 & 0.1651 & -2.646 & 2.392 & 0.9962 & 0.9261 & 2,091 \\
\hline \multirow{3}{*}{0.10} & 50 & 1.098 & 0,1508 & -2.851 & 2.566 & 0,9901 & 0.8766 & 2,106 \\
\hline & 20 & 1.118 & 0,1637 & $-2,557$ & 2,328 & 0,9951 & 0,9201 & 2,118 \\
\hline & 10 & 1.153 & 0.1803 & $-2,617$ & 2,293 & 0.9925 & 1,0490 & 2,059 \\
\hline
\end{tabular}

1 Valores estimados no ajuste da reta : $\Phi^{-1}(F(\triangle))=a+b^{1 / 3}$

2 Valores estimados pelas equações (6.27)

Tabela 11 - Alguns resultados da distribulção simulada de $\Delta_{0}$ para diversos valores de $n_{9}$ e $m_{3}$ e $k=3$

\begin{tabular}{|c|c|c|c|c|c|c|}
\hline & & & Parâme & de $\Delta_{0}$ & & \\
\hline$\Pi_{3}$ & $m_{3}$ & media & variancia & razão & $\lambda^{1}$ & $v^{1}$ \\
\hline \multirow{3}{*}{0,50} & 50 & 1.865 & 3.310 & 1.774 & 0.8870 & 2,105 \\
\hline & 20 & 1.911 & 3.760 & 1.968 & 0.9840 & 1,942 \\
\hline & 10 & $1.87 \mathrm{e}$ & 3,219 & 1.720 & 0.8600 & 2,177 \\
\hline \multirow{3}{*}{0,30} & 50 & 1.930 & 3.952 & 2,048 & 1,0243 & 1,885 \\
\hline & 20 & 1,931 & 3,478 & 1.801 & 0.9015 & 2.144 \\
\hline & 10 & 1.944 & 3,756 & 1.932 & 0,9667 & 2.012 \\
\hline \multirow{3}{*}{0,10} & 50 & 1.850 & 3,113 & 1.683 & 0.8415 & 2,198 \\
\hline & 20 & 1.958 & 3,943 & 2,014 & 1,0075 & 1.944 \\
\hline & 10 & 2.161 & 4,702 & 2.175 & 1,0875 & 1.987 \\
\hline
\end{tabular}

1 Valores estimados a partir de $\lambda=\sigma^{2} /(2 \mu)$ e $\nu=\mu \lambda$ 
Tabela 12 - Distribuição acumulada da simulação de $\Delta_{0} /(2(n-2) / n)$ (ID). distribuị̧̧ăo acumul ada de uma qui-quadrado com $x /(\pi-2)$ g. 1 . (QUID) e distribiçăo acumul ada de uma qui-quadrado com $k-1$ g.1. (OI) no caso de 3 tratamentos.

\begin{tabular}{|c|c|c|c|c|c|c|c|}
\hline \multirow[b]{3}{*}{$m_{3}$} & \multirow[b]{3}{*}{ ID } & \multicolumn{6}{|c|}{$\Pi_{9}$} \\
\hline & & \multicolumn{2}{|c|}{0.50} & \multicolumn{2}{|c|}{0,30} & \multicolumn{2}{|c|}{0.10} \\
\hline & & OI & QUID & OI & QUID & OI & QUID \\
\hline \multirow[t]{6}{*}{50} & 0,8000 & 0.8739 & 0.8033 & 0,8754 & 0,8055 & 0.8752 & 0,8052 \\
\hline & 0,8500 & 0,9180 & 0,8673 & 0,9141 & 0,8615 & 0.9149 & 0,8627 \\
\hline & 0.9000 & 0.9411 & 0.9022 & 0.9480 & 0,9128 & 0.9452 & 0.9084 \\
\hline & 0,9500 & 0.9747 & 0,9553 & 0,9819 & 0,9672 & 0,9793 & 0,9629 \\
\hline & 0.9750 & 0.9884 & 0,9782 & 0,9925 & 0,9854 & 0,9901 & 0,9813 \\
\hline & 0.9950 & 0,9991 & 0.9978 & 0,9996 & 0,9989 & 0.9966 & 0.9930 \\
\hline \multirow[t]{6}{*}{20} & 0,8000 & 0,8747 & 0.8045 & 0.8859 & 0.8205 & 0,8907 & 0.8273 \\
\hline & 0,8500 & 0,9208 & 0,8715 & 0.9197 & 0.8699 & 0.9238 & 0.8760 \\
\hline & 0,9000 & 0.9525 & 0.9198 & 0,9510 & 0,9175 & 0.9530 & 0,9206 \\
\hline & 0.9500 & 0,9851 & 0.9726 & 0,9768 & 0,9587 & 0,9834 & 0,9697 \\
\hline & 0,9750 & 0,9945 & 0,9890 & 0,9922 & 0,9850 & 0.9924 & 0,9854 \\
\hline & 0,9950 & 0,9992 & 0,9980 & 0,9983 & 0,9963 & 0,9996 & 0,9990 \\
\hline \multirow[t]{6}{*}{10} & 0,8000 & 0,8665 & 0,7930 & 0,8864 & 0,8211 & 0.8939 & 0.8321 \\
\hline & 0,8500 & 0,8983 & 0,8384 & 0,9237 & 0.8758 & 0,9314 & 0,8874 \\
\hline & 0.9000 & 0,9481 & 0.9130 & 0,9544 & 0,9228 & 0,9598 & 0.9312 \\
\hline & 0,9500 & 0,9774 & 0,9596 & 0,9795 & 0.9631 & 0.9905 & 0.9812 \\
\hline & 0.9750 & 0.9906 & 0,9820 & 0,9922 & 0,9850 & 0,9972 & 0.9942 \\
\hline & 0,9950 & 0,9981 & 0,9958 & 0,9992 & 0,9982 & 0,9996 & 0.9989 \\
\hline
\end{tabular}


8. O ALGORITMO AID

8.1. Descrição do algoritmo AID

As soluções do algoritmo AID podem ser identificadas através do conjunto $T(I, \gamma)$, uma vez que o algoritmo um caso particular do procedimento de divisão dicotómica hierárquica descrito no Capítulo 2 .

De uma maneira geral tem-se $N$ observações da $(p+1)$-upla $\left(y, x_{1}, x_{2}, \ldots, x_{p}\right)$ que correspondem aos valores da variável dependente $y$ e um conjunto de preditores categorizados $X_{1}, X_{2}, \ldots, X_{p}$, bem como 0 conjunto $I=(1,2, \ldots K\rangle$ de caselas não vazias resultantes da tabulação cruzada total das observaçzes. Quanto à regra de divisão, o algoritmo AID associa a cada variável $X_{p}$, uma regra $\gamma_{p}, p=1, \ldots, P$, que pode ser tanto a regra monotônica como a livre.

O algoritmo se desenvolve através de divisões dicotómicas nas caselas marginais. Seja $I\left(X_{\mathrm{p}}\right)=$ $\left(1,2, \ldots, k_{p}\right\}$ o conjunto de indices marginais criados pelo preditor $X_{p}$ sobre todo o conjunto $I$. Então, $\Psi\left(I\left(X_{p}\right), \gamma_{p}\right)$ é - conjunto de todas as possiveis partiçós dicotómicas de $I\left(X_{p}\right)$ com relação a $\gamma_{p}$. Como $I\left(X_{p}\right)$ é uma particular partição do conjunto $I$, então $\Psi\left(I\left(X_{\mathrm{p}}\right), \gamma_{\mathrm{p}}\right)$ também corresponde a todas as partiçós dicotomicas do conjunto I com a regra marginal $\gamma_{\mathrm{p}}$. Logo a classe de todas as 
partiçes dicotomicas possiveis do conjunto $I$ no algoritmo AID é dada por

$$
\Psi(I, \gamma)=\bigcup_{p=1}^{p} \Psi\left(I\left(X_{p}\right), \gamma_{p}\right)
$$

Ao conjurto I esta associado um conjunto de pesos $W=\left(w_{i}=n_{i} / N: i \in I\right)$, onde $w_{i}$ e a proporção de elementos em cada casela $i \in I$. Para o conjunto $I\left(X_{p}\right.$. tem-se associado um conjunto $w\left(x_{\mathrm{p}}\right)$, que corresponde à proporça de elementos em cada casela marginal do preditor $X_{p}$.

Sendo $i$ um elemento de $T(I, \gamma)$ tem-se que

$$
i=\left(I_{1}(i), I_{2}(i), \ldots, I_{a}(i)\right\}
$$

e portanto de (2.15) tem-se que

$$
\begin{aligned}
& \operatorname{SOT}(\operatorname{total})=\sum_{g=1}^{a} \operatorname{SQT}(g r u p 0)+\sum_{g=1}^{a} N_{g}(\bar{y}-\bar{y})^{2} \\
& =\sum_{g=1}^{a} \operatorname{SQT}(\varepsilon r u p o)+\operatorname{SQX}(G)
\end{aligned}
$$

o algoritmo prossegue fazendo a divisăo tal que:

i) Criterio de selecão: Dos $G$ grupos candidatos seleciona-se aquele que tem maior SQT(grupo). Sem perda de generalidade assume-se que

$$
S Q T_{1}(\text { grupo }) \leq \operatorname{SQT}_{2}(\text { grupo }) \leq \ldots \leq S Q T_{\text {a }}(\text { grupo) }
$$


i) Divisão ótima: Para cada preditor $X_{p}$ encontra-se a partição de $\Psi_{\mathrm{o}}\left(X_{\mathrm{p}}\right), \gamma_{\mathrm{p}}{ }^{\prime}$ que conduz à redução máxima $S Q E$, isto é,

$$
\begin{aligned}
& B\left[\gamma_{p}, I_{G}\left(X_{p}\right), W_{G}\left(X_{p}\right)\right]= \\
& =\max \left(S Q E\left[d, \gamma_{p}, I_{G}\left(X_{p}\right), W_{G}\left(X_{p}\right)\right]: d \in \Psi\left(I_{G}\left(X_{p}\right), \gamma_{p}\right)\right)
\end{aligned}
$$

onde $S Q E\left[d, \gamma_{p}, I_{g}\left(X_{p}\right), W_{G}\left(X_{p}\right)\right]$ significa a redução $S Q E$ na partição $\alpha$, por uma divisão dicotómica do grupo $G$ com o conjunto de indices $I_{G}\left(X_{p}\right)$ do preditor $X_{p}$, usando a regra $\gamma_{p}$, tendo como conjunto associado de pesos $w_{G}\left(X_{p}\right)$.

Seja $x_{0}$ o preditor tal que

$$
\begin{aligned}
& B\left[\gamma_{0}, I_{\mathrm{g}}\left(X_{0}\right), w_{\mathrm{d}}\left(X_{0}\right)\right]= \\
& =\max \left(S Q E\left[\gamma_{\mathrm{p}}, I_{\mathrm{a}}\left(X_{p}\right), W_{\mathrm{a}}\left(X_{\mathrm{p}}\right)\right]: 1 \leq \mathrm{p} \leq \mathrm{P}\right)
\end{aligned}
$$

Então, existe uma partição $\alpha_{0} \in \Psi\left(I_{a}\left(X_{0}\right), \gamma_{0}\right)$ tal que

$$
B\left[\gamma_{0}, I_{0}\left(X_{0}\right), W_{0}\left(X_{0}\right)\right]=\operatorname{SQE}\left[\alpha_{0}, \gamma_{0}, I_{0}\left(X_{0}\right), W_{0}\left(X_{0}\right)\right]
$$

Essa partição define a di visão ótima neste estágio.

ii) As regras de parada: A divisão ocorre efetivamente se as seguintes condições forem preservadas:

$$
\text { - } B\left[\gamma_{0}, I_{G}\left(X_{0}\right), W_{G}\left(X_{0}\right)\right] \text { é maior que algum }
$$

valor pré determinado expresso como uma fração de SQT(total). 
resultantes ef maior que algum número fixado, em geral 25.

- o número total de divisర̃es não alcança um certo máximo, por exemplo $6<30$.

Considerando que a partição final produzida pelo algoritmo AID tem $G$ grupos, isto e, houve G-1 divisões, mostra-se que

$$
S Q \times(G)=\sum_{g=1}^{G-1} B(g)=S Q \times(G-1)+B(G-1)
$$

isto é, a soma de quadrados explicada pelo modelo de $G$ grupos é a soma da redução $S Q E$ nas $G-1$ divisóes dicotômicas que ocorrem para formar a partição.

A primeira regra de parada diz que a redução máxima nessa divisão deve ser maior que uma fração de SQT(total). Assim usando a expressão (8.7), tem-se a estatistica

$$
\begin{aligned}
& R^{2}(O)=S Q \times(G) / S Q T(\text { total }) \\
& =R^{2}(G-1)+E(G-1) / S Q T(t o t a r)
\end{aligned}
$$

que pode ser útil para tal fim, uma vez que se uma nova divisão aumenta um pouco a explicação do modelo então essa divisão não deve ocorrer. Esse valor é escolhido de maneira empirica tendo em mente alguns cuidados. Se ele e fixado muito baixo, podem-se obter divisóes devidas unicamente à aleatoridade dos dados. Se muito alto, pode-se terminar sem ter sido feita qualquer divisăo, e não se sabe se isso é devido a estrutura dos dados ou 
devido ao valor critico escolhido. Por outro lado, um limite inferior único não é razoável para todos os tipos de problemas.

A segunda regra de parada tenta evitar influencias de valores extremos no criterio. Já a terceira é apenas uma medida de segurança para evitar um grande número final de grupos, quando o objetivo primeiro de agrupar pode estar seriamente comprometido.

A procura da partição $\alpha_{0}$ em (8.8) năo leva em conta a regra $\gamma$ associada ao preditor e nem o número de classes ou mesmo a distribuição dos tamanhos das caselas. Sabe-se atualmente que esses fatores são importantes na determinação do comportamento de $B$, uma vez que as divisões tendem a ocorrer nos fatores com o maior número de classes e com a regra de divisão livre, em vez da monotônica.

8.2. Algumas alternativas sobre critérios de seleção e regras de parada

Como o algoritmo AID possui alguns pontos que podem tornálo pouco atrativo na sua aplicação em determinados casos, BUSSAB (1976) sugeriu algumas modificaçães que podem ser utilizadas para melhorar o seu desempenho. o critério de seleção e as regras de paradas influem de fato na obtenção da árvore de divisão. Em muitas situaçóes é recomendado que se apresente a análise com as diversas combinações de critério de seleção e regras de parada para que o pesquisador escol ha a árvore que possa melhor explicar o agrupamento, de acordo com a 
teoria especifica do assunto em questão.

No algoritmo AID, o critério de seleção elege como o grupo a ser dividido aquele onde se da a maior redução da soma de quadrados entre grupos SQE. Entretanto isto implica que o candidato é aquele grupo que possui a maior soma de quadrados total que pode ser expressa por

SQT $\operatorname{g}_{\text {grupo }}=\sum_{k \in g j=1} \sum_{k}^{n_{k}}\left(y_{k j}-\bar{y}_{g}\right)^{2}=\sum_{i=1}^{N_{g}}\left(y_{i g}-\bar{y}_{g}\right)^{2}$

Um rápido exame nessa expressão mostra que el a aumenta com o número de elementos no g-ésimo grupo, $N_{g}$. Assim um grupo grande pode produzir uma SQT(grupo) maior que outro grupo que possui grande diferença entre as médias de caselas.

Uma abordagem alternativa proposta por BUSSAB (1976) é usar uma divisão do passo seguinte para selecionar o grupo que realmente produz a maior redução SQE. Assim em cada conjunto $I_{g}(d)$ da partição d encontra-se o correspondente valor do criterio $B C I_{g}$, e o grupo candidato será aquele, $\varepsilon_{0}$, tal que

$$
B\left[I_{0}(d)\right]=\max \left\{B C I_{g}(\alpha): 1 \leq g \leq 0\right\}
$$

Outro critério de seleção de partição ótima é aquele que escolhe a partição que apresenta o menor nível descritivo da estatistica $B$. Quando, entretanto, esta última não tem distribuição conhecida, pode-se usar o critério alternativo (BUSSAB, 1976) que algumas vezes é também chamado de critério empírico 
(Ho, 1987), o qual através do coeficiente de explicação $R^{2}$ definido no Capitulo 2 , busca a partição que possui o maior $R^{2}$, ou seja a partiçăo mais significativa. A relação entre os criterios de seleção que se utilizam da estatistica $B$, e do coeficiente de explicação $R^{2}$ ou mesmo $R_{*}^{2}$ parece muito grande, uma vez que quase sempre levam ao mesmo resul tado.

A proposta de KASS (1980), escolhe a partição que apresenta o menor nivel de significância de Bonferroni. Este úlimo, definido a partir da desigualdade de Bonferroni, considera que se o valor da estatistica $B$ for maior que o valor crítico a um nível $\alpha D$, então a divisão deve ser feita, onde $D$ é igual a $K-1$ se a regra de partição for a monotonica, e igual a $e^{K-1}-1$ se a regra de partição for a livre, e a é um nível de significância padrăo pré-fixado.

Tanto no critério do menor nível de significância de Bonferroni, como no criterio de menor nível descritivo, o conhecimento da distribuição da estatistica $B$ é muito importante e na maioria das vezes se utiliza sua distribuiça assintótica.

8. 3. Um procedimento para valores pequenos de $B$

A estatistica do teste da razão de máxima verossimilhança para testar a hipótese nula de que todas as médias $5 \tilde{a} 0$ as mesmas contra a alternativa de que elas podem ser divididas em dois grupos $e$ dada por $B / \sigma^{2}$, para $\sigma^{2}$ conhecido, como foi visto no Capítulo e. A rejeiçăo da hipótese nula e o valor observado da estatística são 
elementos suficientes para mostrar como os grupos podem ser divididos. Levando também em consideraçăo, conforme mostrado por BUSSAB (1976), que para valores menores que 30, a aproximação à distribuição de qui-quadrado pode ser muito útil e prática para se obterem os valores da probabilidade de $B / \sigma^{2}$, o algoritmo AID pode então ser redefinido usando um procedimento de teste hierárquico.

Vale a pena lembrar que o teste da razão de máxima verossimilhança acima mencionado fol obtido para grandes amostras ou onde a variancia $\alpha^{2}$ é conhecida. Assim poderia ser usada a estatistica $s^{2}=5 Q 0(t o t a l) /$ como estimador de $\sigma^{2}$ para problemas onde se tem um grande número de graus de liberdade do residuo.

Outro fato, visto anteriormente, é que a aproximação (6. 26$)$ para um dado conjunto de parâmetros $(\gamma, K, W)$ tem associada a linha reta

$$
{ }^{-1}[F(b: \gamma, K, W)]=M(\gamma, K, W) b^{1 / 3}+N(\gamma, K, W)
$$

que torna possivel a comparação de valores para diferentes conjuntos de parâmetros.... 0 valor mais significante é aquele com o maior valor de $\Phi^{-1}$. 'A quantidade $b$ aqui mostrada e o valor observado de $B / O^{2}$.

Um terceiro ponto se refere ao uso da desigualdade de Bonferroni. Para uma sequencia de $T$ testes de hipóteses cada um com nivel de significância menor ou igual a $\alpha$, o nível geral de significância é da ordem de $a^{*}=\alpha / T$. Como no procedimento aqui descrito não se sabe "a priori" quantos testes serão feitos durante o processo, então recomenda-se, em cada passo, mudar o nivel de significância para testar cada suposição em 
particular.

Dessa maneira os passos do algoritmo AID podem ser redefinidos. Após $G-1$ divisões sucessivas, o processo produz uma partição $i$ com $G$ grupos.

$$
i=\left(I_{1}(i), I_{2}(i), \ldots, I_{0}(i)\right\}
$$

e os passos do algoritmo AID tornam-se:

i) Critério de seleção: Dos $G$ conjuntos da partição $i$, seleciona-se como grupo aquele que produz o valor mais significamte quando dividido. Sem perda de generalidade seja $I_{a}(i)$ tal grupo.

ii) Divisão ótima: Para cada preditor $X_{p}$, encontrar a partição de $\left.\Psi_{a}\left(I_{p}\right), \gamma_{p}\right)$ que leva a redução máxima $S Q E$, $B\left[\gamma_{p}, I_{G}(X), W_{\mathrm{p}}\left(X_{\mathrm{p}}\right)\right]=$ $=\max \left(\operatorname{SQE}\left[d_{s} \gamma_{\mathrm{p}}, I_{\mathrm{o}}\left(X_{\mathrm{p}}\right), W_{\mathrm{o}}\left(X_{\mathrm{p}}\right)\right]: d \in \Psi\left(I_{\mathrm{a}}\left(X_{\mathrm{p}}\right), \gamma_{\mathrm{p}}\right)\right)$

Sendo $X_{p}$ o preditor que produz o valor mais significante, isto $e$,

$\Phi^{-1}\left[F\left(z: \gamma_{0}, I_{0}\left(X_{0} 3, W_{0}\left(X_{p}\right)\right\}\right]=\right.$

$$
=\max \left\{\Phi\left[F\left(b: \gamma_{p}, I_{g}\left(X_{p}\right), W_{d}\left(X_{p}\right)\right\}\right]: 1 \leq p \leq P\right\}
$$

e $B_{\mathrm{a}} S_{\mathrm{a}}$, a redução SOE na G-ésima divisão, então existe uma partição $d_{0} \in \Psi\left(I_{0}\left(X_{0}\right), \gamma_{0}\right)$ tal que 


$$
\left.B C S_{a}\right)=\operatorname{SQE}\left[\alpha_{0}, \gamma_{0}, I_{a}\left(X_{0}\right), W_{a}\left(X_{0}\right)\right]
$$

Essa partição dicotómica $d_{0}$ é a divisão ótima nesse estágio.

iii) A regra de parada: A divisão dicotómica ocorre se todos os valores observados de $B\left(S_{g}\right)$, para $1 \leq \mathbb{E} \leq G$, são significantivos ao nivel $\alpha / b$, com $\alpha$ fixado "a priori".

As tabelas construidas por BUSSAB (1976) podem ser utilizadas pois trazem os pontos criticos de $\Phi^{-1}(F)$ com valores crescentes de $G$, usando a aproximação (6. 26) para a distribuição de qui-quadrado tendo um nível de significancia geral de $5 \%$.

8. 4. Procedimentos para valores grandes do criterio $B$

As sugestōes da seç̃o anterior não são aplicáveis quando os valores de $B / S^{2}$ são grandes e em geral isso pode ocorrer para amostras grandes e um número grande de preditores.

Como a aproximação (6.26) para a distribuição de qui-quadrado não é boa para valores extremos na cauda superior da distribuição, é conveniente se utilizarem outros critérios nessa situação.

Fara a regra de parada, por exemplo, uma nova divisăo ocorre se sua contribuiçăo, a explicaçăo $R^{2}$ do modelo, dada por SQE/SQT(total) é significativa, tendo sido fixada uma proporção minima arbitrária Fr[min] da soma de quadrados total que cada divisão pode explicar. 
Como não é razoável se fixar "a priori" um número sem levar em conta a natureza e estrutura dos dados, parece razoável se utilizar essa medida padronizada. Assim pode-se julgar a significância de uma divisão pela estatistica (5.3) que neste caso corresponde a

$$
\begin{aligned}
R_{*}^{2}(G) & =S Q \times(G) / S Q E(\text { total }) \\
& \left.=R_{*}^{2}(G-1)+E(G-1) / \text { SQE(total }\right)
\end{aligned}
$$

Como ate o momento parecem não existir evidéncias suficientes para valores empíricos razoáveis em problemas práticos, existe a sugestão de escolher um limite inferior, baseado na ocorrencia de divisóes devidas apenas a aleatoridade dos dados.

A variável aleatória $B\left(\gamma_{L}, W_{O}\right)$ obtida quando da aplicaça da regra de divisão dicotômica livre e igual número de observaçóes por casela produz uma função distribuição acumulada que pode ser considerada como um limite inferior dos demais casos, conforme mostrado anteriormente. Usando essa propriedade da variável $B\left(\gamma_{L}, W_{0}\right)$, além da desigualdade de Eonferroni para a obtenção do nivel de significância geral da sequência de $T$ testes de hipóteses do processo de divisão, BUSSAB (1976) construiu uma tabela para a quantidade $b_{a}(\alpha, T)$, denotada por $\hat{b}_{0}(\alpha, T)$, obtida a partir da expressão 


$$
P\left(B\left(\gamma_{L}, W_{0}\right) \leq b_{0}(\alpha, T)\right]=1-\alpha / T
$$

para o caso em que o número máximo de caselas por preditor e 10 e o nível de significancia geral é 0,05 .

A ligação entre o valor observado de $b_{0}(\alpha, T)$ e a proporção mínima Pr(min) ou $\operatorname{Pr}_{*}(m i n)$ pode ser feita julgando a significancia de $\left.\vec{b}_{0} C \alpha, T\right) \sigma^{2}$.

Como o teste da razão de verossimilhança foi obtido para $\alpha^{2}$ conhecido ou para valores grandes de $\nu$ quando $\sigma^{2}$ estimado por $s^{2}=S Q D(t o t a l) /$, a proporçăo mínima da soma de quadrados total no criterio AID é escolhida de tal modo que

$$
\begin{array}{r}
\operatorname{Pr}(\min )=B / \text { SQT(total) } \geq b_{0}(\alpha, T) s^{2} / \text { SQT }(\text { total }) \\
\left.=\left(b_{0}(\alpha, T), \nu\right) \times(\text { SQDCtotal }), \text { SoT }(\text { total })\right)
\end{array}
$$

sendo garantido que toda di visão produz um modelo com uma significância geral menor que $\alpha$.

8.5. Uma boa aproximação para a distribuição de $\mathrm{B}_{\mathrm{K}}$

Para o caso em que a regra de divisão é a livre, uma boa aproximação para a distribuição da estatistica $B$ quando da aplicação da técnica AID, foi 
obtida por SCOTT \& KNOTT (1976), baseada em resultados de BUSSAB (1976) e KASS (1975).

Este último considerou a distribuição de permutação da estatistica $B$ para a técnica AID e tabulou diversos pontos de porcentagem da distribuição limite quando $n \rightarrow \infty$ no caso particular em que o número de observaçöes por casela, $n_{i}$, é o mesmo.

A aproximação de SCOTT \& KNOTT (1976) estende o resultado para o caso em que o número de observaçöes por casela é diferente.

Supondo que $k / n$ permanece $f i x o$ quando $k \rightarrow \infty$ e $w=2+\alpha k / n$, onde $\alpha=\left\langle\sum_{1}^{K} \sqrt{w_{i} / K}\right)^{2}$ e $w_{i}=n_{i} / n$, de tal modo que $\max w_{i} \rightarrow 0$ e nenhuma categoria está vazia, a variável $B$ tem aproximadamente distribuiça qui-quadrado com $\nu_{0}$ graus de liberdade e coeficiente de proporcionalidade igual a $2(1-w / \pi)$ e com $\nu_{0}=a k /(\pi-\omega)+1 / 2$.

No estudo da precisão dessa aproximação, SCOTT \& KNOTT (1976) verificaram que ela e muito boa, sendo que na proximidade do quantil $95 \%$ ela é melhor, e esses resul tados também são válidos para $k$ pequeno.

8.6. Uma modificação do algoritmo AID para dados da familia exponencial.

Considerando a estatistica $\Delta \hat{\mu}_{d}{ }^{2}$ como medida de homogeneidade entre grupas, o algoritmo AID pode ser aplicado a diferentes tipos de variaveis aleatórias, atendendo, pelo menos, às distribuiçóes que 
pertençam à familia exponencial. Desse modo o algoritmo pode ser redefinido por:

Passo 1: Critérios de seleça:

Como critério de seleçăo pode-se utilizar um dos seguintes:

a escolher como grupo a ser dividido aquele onde se dá a maior redução $\Delta\left(\hat{\mu}_{d}\right)$.

ii) escolher como grupo a ser dividido aquele que produz no passo seguinte a maior redução $\Delta(\hat{\mu})$.

Passo 巳: Critérios de partição ótima.

Como criterios de partição otima têm-se: i) escolher como divisão ótima aquela que produz a mai or redução $\Delta\left(\hat{\mu}_{d}\right)$.

ii) escolher como divisão ótima aquela que produz a redução $\Delta\left(\hat{\mu}_{\mathrm{d}}\right)$ com o menor nivel descritivo.

Passo 3: Regras de parada.

Como critérios de parada têm-se:

i) o valor de $R^{2}(5.4)$ ou de $R_{*}^{2}(5.5)$ se alterou menos que um valor pré-fixado com relaçăo à divisão imediatamente anterior.

ii) o número de elementos nos grupos resultantes é menor que algum numero pre-fixado.

iii o número total de divisóes não alcança um certo maximo.

w) o nivel descritivo do maximo da reducăo $\Delta\left(\hat{\mu}{ }_{d}\right)$ 
150.

não é maior que um valor préfixado. 


\section{A UTILIZAÇÃO DO ALGORI TMO AID EM DADOS BINOMI AIS}

A combinação entre os diversos criterios de seleção, os diversos tipos de partição ótima e as diversas regras de parada, em geral, levam a diferentes árvores de agrupamento. Cabe ao pesquisador escolher a árvore que mel hor possa explicar-o agrupamento.

A aplicação das alternativas onde se faz necessário o conhecimento da distribuição do máximo da estatistica $\Delta\left(\hat{\mu}_{d}\right)$ pode tornar o algoritmo pouco viável.

Para o caso normal, fez-se um breve apanhado no Capítulo 6 e o Capítulo 7 apresentou um

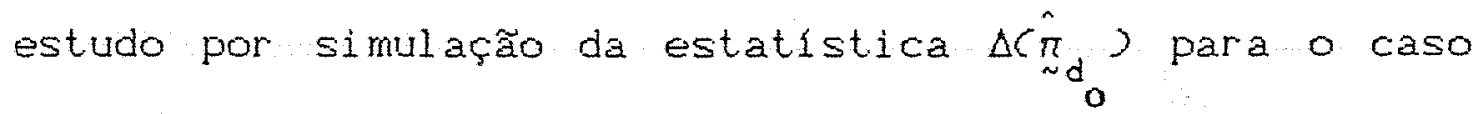
bi nomial.

Apresenta-se, a seguir, a análise do experimento sobre o efeito de 12 fungicidas em porcentagem de germi nação de sementes de milho, ci tado no Capitulo 1, considerando o modelo logito, bem como a aplicação de técnicas de agrupamentos. 
9.1. Uma análise de um experimento com fungicidas.

$\mathrm{Na}$ Tabela 13 estão os resultados de um ensaio, inteiramente ao acaso, sobre o comportamento de 12 fungicidas em porcentagem de germi nação de sementes de milho, com 4 repetições, realizado no Departamento de Agricultura da ESALQ/USP.

Tabela 14 - Porcentagem de germinação de sementes de milho em um ensaio completamente ao acaso sobre o comportamento de 12 fungicidas com 4 repetições.

\begin{tabular}{rllll}
\hline & \multicolumn{4}{c}{ REPETI ÇES } \\
FUNGICIDAS & 1 & 2 & 3 & 4 \\
\hline 1 & 88 & 94 & 88 & 88 \\
2 & 95 & 97 & 86 & 89 \\
3 & 91 & 90 & 94 & 92 \\
4 & 95 & 86 & 96 & 94 \\
5 & 94 & 92 & 93 & 89 \\
6 & 85 & 80 & 85 & 83 \\
7 & 80 & 82 & 74 & 83 \\
8 & 94 & 84 & 72 & 76 \\
9 & 90 & 83 & 90 & 91 \\
10 & 95 & 95 & 96 & 95 \\
11 & 96 & 99 & 92 & 95 \\
12 & 94 & 91 & 89 & 95 \\
\hline
\end{tabular}

Fonte: Departamento de Agricultura, ESALQ - USP.

- programa, bem como sua saída, para a análise estatística utilizando-se o modelo, em que se supōe distribuição binomial para a variavel resposta, 
número de sementes germinadas, e a transformação logistica para o valor esperado, encontram-se no Apéndice 3.

A análise do desvio correspondente CTabela $14)$ indica que existem diferenças entre fungicidas, pois a razão dos desvios médios quando comparada com uma distribuição $F$ com 11 e 36 graus de liberdade tem um nivel descritivo inferior a $1 \%$.

Tabela 14 - Quadro de análise do desvio para o número de sementes germinadas em um ensaio completamente ao acaso sobre o comportamento de 12 fungicidas com 4 repetições.

\begin{tabular}{ccccc}
\hline FONTE DE VARI AÇAO & GL & DESVIO & DESVIO MEDIO & RAZÃO \\
\hline FUNGICIDAS & 11 & 173,87 & 15,81 & 10,47 \\
RESIDUO & 36 & 54,34 & 1,51 & \\
\hline
\end{tabular}

Os gráficos de residuos não indicaram qualquer viol ação contra o modelo adotado, quer seja com relação à função de iigação, quer seja com relaçăo à função de variância. Pode-se dizer que o modelo usado está bem ajustado aos dados.

No Gráfico 1 estão os valores observados de porcentagem de sementes germinadas e os respectivos valores ajustados para os 12 fungicidas. 


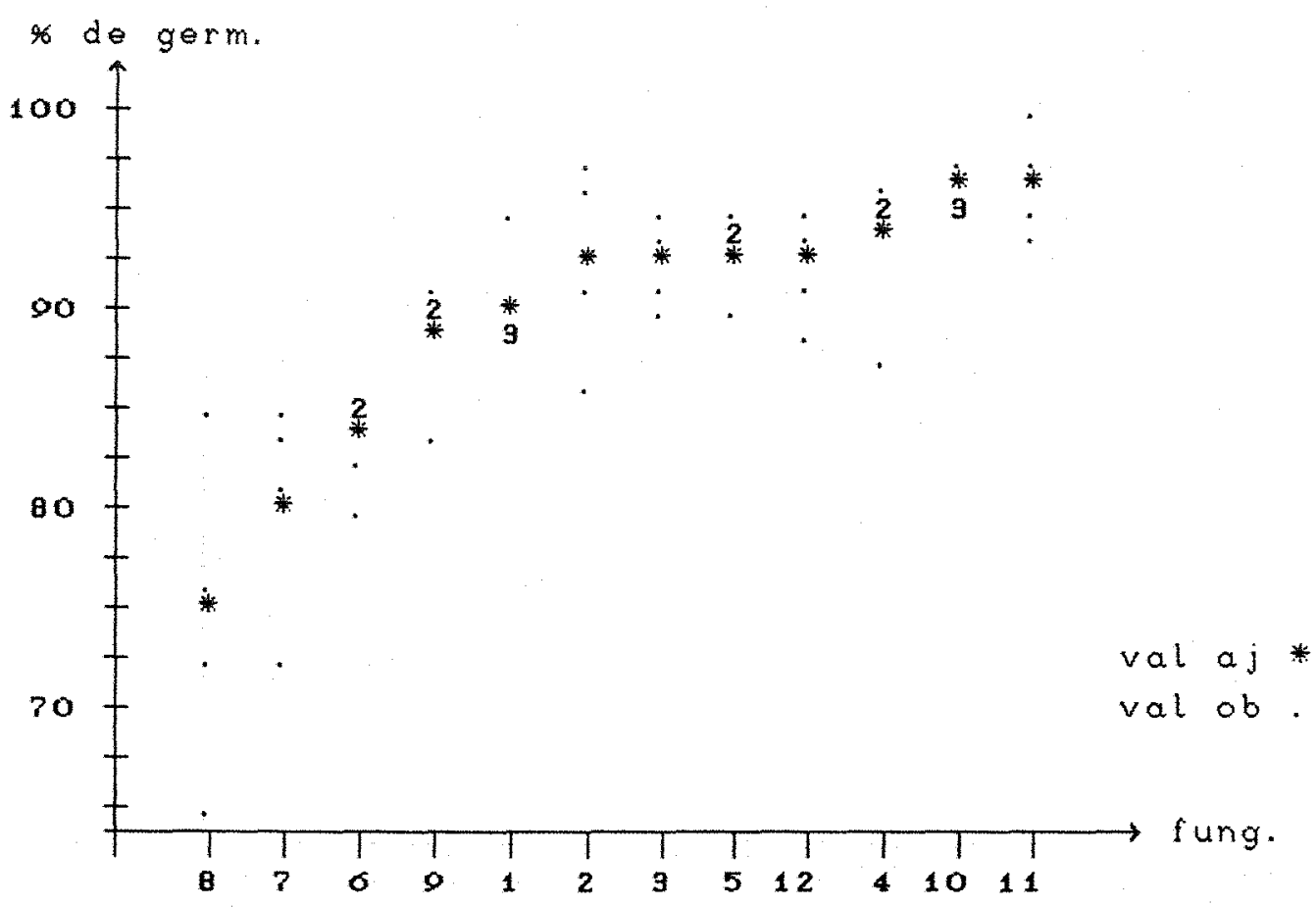

Gráfico 1 - Porcentagem de germinação de sementes de milho observada e ajustada pelo modelo com com ligação logística por fungicida.

Também fol ajustado o modelo com ligação 'probit', que apresentou resultados bem próximos aos do modelo com ligação logística. Entretanto, este último é de mais fácil interpretação.

9.2. Resultados da aplicaça do algoritmo AID

Na Tábela 15 estão os principais elementos para a construção de árvores de divisão dicotômica. A combinação dos diferentes criterios de seleção, criterios de partição ótima e regras de parada pode produzir 
155.

agrupamentos diferentes.

Tabela 15 - Quadro resumo dos resultados do algoritmo de divisão dicotômica hierárquica.

\begin{tabular}{|c|c|c|c|c|c|}
\hline no & $\Delta_{0}$ & $\lambda \times \Delta_{0}$ & $v$ & $\hat{\alpha}$ & grupos \\
\hline 1 & 139,6 & 192.1 & 10,51 & $<<0,1 \%$ & $\begin{array}{c}\{8,7,6\rangle \text { e } \\
\{9,1,2,3,5,12,4,10,11\}\end{array}$ \\
\hline 2 & 8,825 & 12,14 & 2,628 & $0,49 \%$ & $\{8\}$ e $\{7,6\}$ \\
\hline 3 & 16,62 & 22,86 & 7,884 & $0,34 \%$ & $\{9,1,2,3,5,12,4\}$ e $\{10,11\}$ \\
\hline 4 & 6,585 & 9,060 & 6,132 & $17,87 \%$ & $\{9,1\}$ e $\{2,3,5,12,4\}$ \\
\hline 5 & 0,028 & 0,039 & 1.752 & $95,17 \%$ & $\{10\}$ e $\{11\}$ \\
\hline 6 & 1,627 & 2,239 & 1,752 & $27,58 \%$ & $\{7\}$ e $\{6\}$ \\
\hline
\end{tabular}

Considerando como criterio de seleção a maior redução $\Delta\left(\hat{\pi}_{d}\right)$, como partição ótima o ramo que no passo seguinte apresenta o menor nivel descritivo e como regra de parada, a partiç̃o otima com um nivel descritivo superior a um valor preffixado, obteve-se a arvore de divisão dicotômica do Gráfico 2 .

A formação da arvore começou pelo nó (1) com o cálculo da estatística $\lambda \times \Delta_{0}$ cujo valor foi de 192,1 e o número de graus de 1 iberdade associado é de $v=10,51$ e - nivel descritivo é $\hat{\alpha}<<0,1 \%$. Para a escolha da próxima partição dicotómica, calculou-se o valor da estatística $\lambda \times \Delta_{\text {o }}$ no passo seguinte: para o no (2) seu valor fol de 12,14 com $\nu=2,628$ e nivel descritivo de $\alpha \bumpeq 0,49 \%$ e para o no $(3), \lambda_{x} \Delta_{0}=22,86 \operatorname{com} \nu=7,884$ e $\alpha 20,34 \%$ Desse modo a segunda divisão escolhida deve ser feita no no (3). Como - nivel descritivo e inferior a $1 \%$, examinam-se as partiçoses no passo seguinte: para o no $(4), \lambda \times \Delta,=9,060$ 
com $\nu=6,132$ e o nivel descritivo de $\hat{\alpha} \sim 17,87 \%$ e para o nó (5), $\lambda \times \Delta_{0}=0,039$ com $\nu=1,732$ e o nivel descritivo de $95,1 \%$. Com esses resultados chegou-se à conclusão que esse nó não deve ser dividido. Ao se examinar o nó (2), deve-se aceitar sua divisão em dois grupos e passa-se a examinar o nó (6). O valor de $\lambda \times \Delta_{0}$ e de $2,239 \mathrm{com} 1,752$ graus de liberdade e o nivel descritivo de $27,58 \%$. Portanto o nó ( 6$)$ não deve ser dividido.

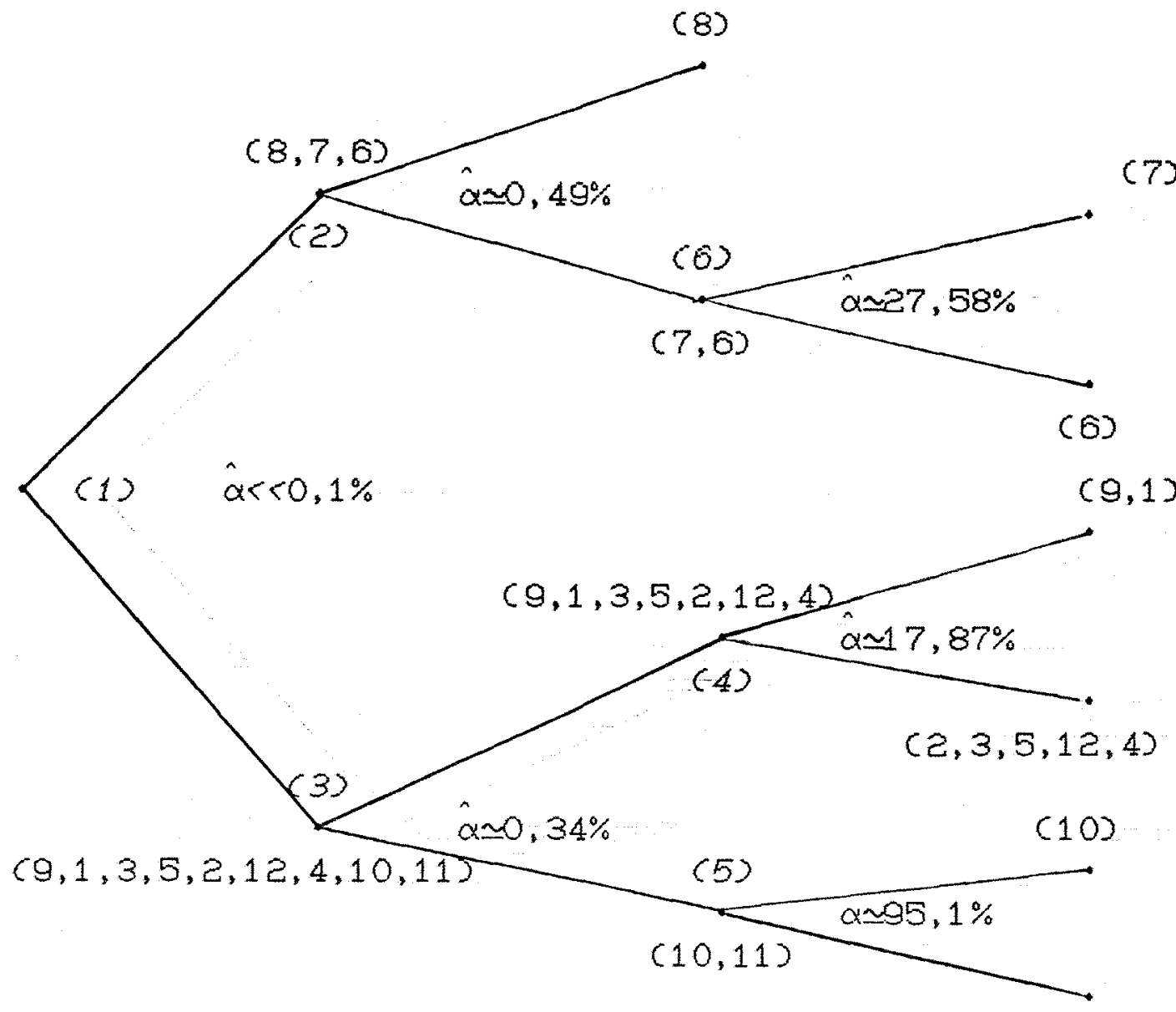

Gráfico 2 - Arrvore de divisăo dicotômica considerando como partição ótima o menor nivel decritivo no passo seguinte. 
157.

Ao final do algoritmo obtiveram-se os grupos: $\{8\},\{6,7\},\{9,1,3,5,2,12,4\},\{10,11\}$. Pode-se dizer que adotando-se o nivel de significancia individual de $1 \%$, o nivel geral é de $1-(1-0,01)^{5}=4,9 \%$.

- Gráfico 3 foi montado considerando como critério de seleção o grupo onde se dá a maior redução, como criterio de partição ótima a que produz a maior redução e como regra de parada a obtenção de 5 grupos finais, o algoritmo começa no nó (1) da Tabela 15. A seguir, examinando os nós (2) e (3), este último é escolhido. Até este passo têm-se 3 grupos. 0 algoritmo prossegue examinando o nó (2). A seguir viria o nó (4). Os grupos formados são: $\{8\},\{7,6\},\{9,1\},\{2,3,5,12,4\}$, $\{10,11\}$.

$\mathrm{Na}$ primeira aplicação do algoritmo, a estatistica $R^{2}$ é da ordem de $(139,6+8,825+16,62)$, $228,21=72,32 \% \mathrm{Na}$ segunda aplicação a estatistica $R^{2} \in$ $\operatorname{de}(139,8+8,825+16,62+6,585) / 228,21=75,21 \%$

Com relação à estatistica $R_{*}^{2}$, na primeira aplicação seu valor foi de $(139,6+8,825+16,62)$ ' $173,87=94,92 \%$ e na segunda aplicação $(139,6+8,825+$ $16,62+6,585)<173,87=98,71 \%$.

Essas duas combinações de critérios usados no algoritmo AID ilustram diferentes maneiras de se construir árvore de di visão dicotômica hierárquica, sendo que a última dispensa totalmente o uso da distribuição da estatistica $\left.\Delta \hat{\pi}_{\sim_{0}}.\right)$. 
158.

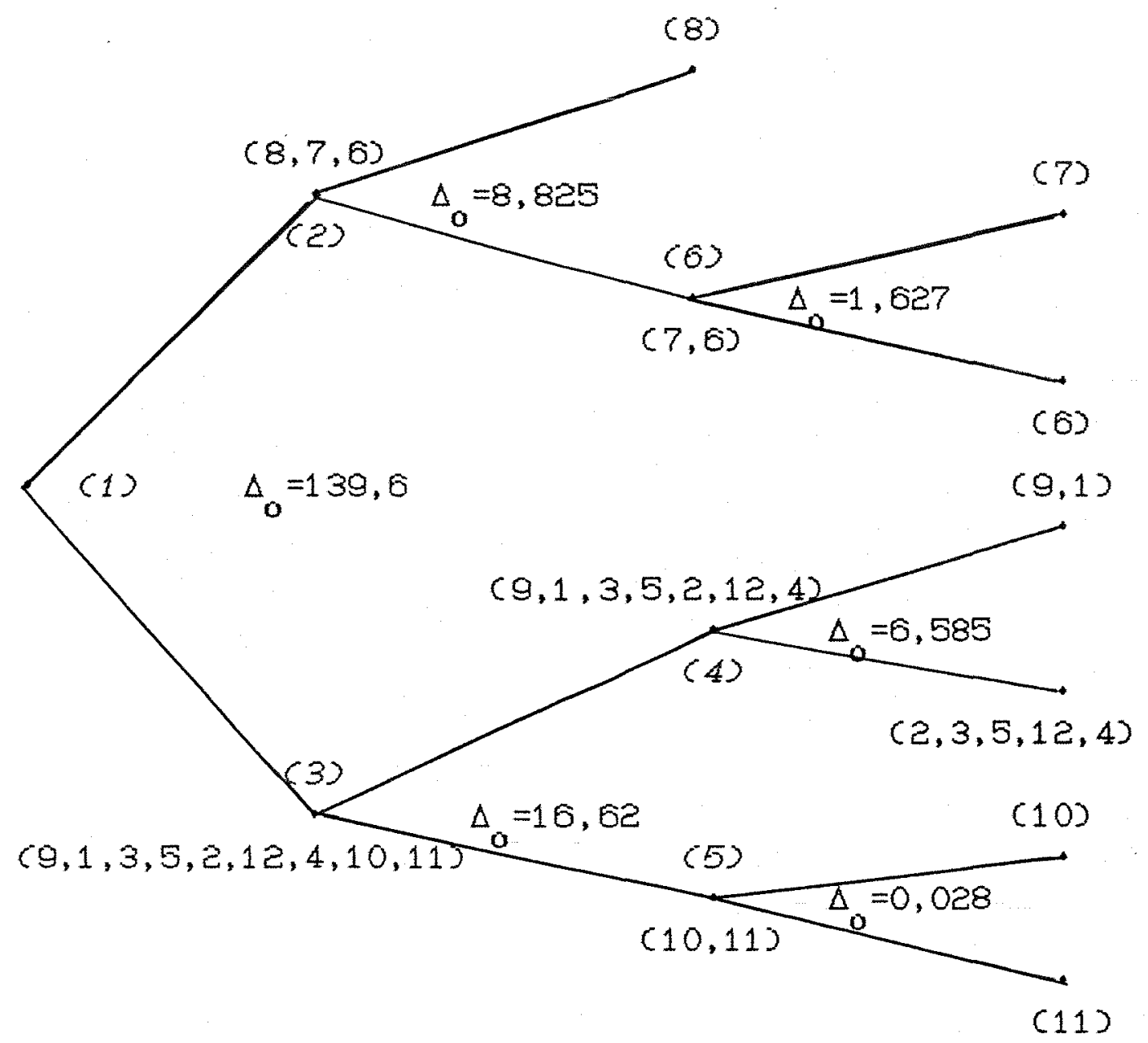

Gráfico 3 - Arvore de divisão dicotômica considerando como partição ótima a maior redução e como regra de parada a obtenção de $G$ grupos 
159.

10. CONCLUSOES.

Para problemas em que não é conveniente assumir que os dados venham de uma distribuiçăo normal, a aplicação direta do algoritmo AID pode tornar-se pouco atrativa. Usando, então, a funçăo desvio definida em (3.6) póde-se generalizar o conceito de medida de homogeneidade entre grupos utilizando a estatistica $\Delta\left(\hat{\mu}_{d}\right)$, sempre que os dados sejam de uma distribuiçăo que pertence à familia exponencial.

As expressões para $\Delta\left(\hat{\mu}_{d}\right)$ foram obtidas considerando as funções de ligações naturais, peso "a priori" unitário e as distribuições de erro binomial, Poisson e normal. Para esta última os resultados coincidem com os obtidos por SCOTT \& KNOTT (1974) e BUSSAB (1976). Com relação à proporção explicada do desvio, $\mathrm{R}^{2}$, dada em (5.4), bem como para a proporfăo explicada do desvio padronizada, dada em (5.5), também foram obtidas as express̃es, sob as mesmas condições. Novamente os resultados coincidiram com os obtidos por BUSSAB (1976) no caso de distribuiçăo normal.

Por simulação, verificou-se que o máximo da redução $\Delta \hat{\mu}_{\sim}{ }^{3}$, que corresponde a dados com distribuição binomial, tambem apresenta distribuiçăo aproximadamente proporcional a uma distribuiçăo qui-quadrado com $k /(\pi-2)$ graus de liberdade e parametro 
de proporcionalidade igual a $\pi / 22(\pi-2)]$.

Esses resultados foram verificados para $K$ variando de 3 a 10 , sendo que a qualidade da aproximação é maior conforme $K$ aumenta.

Também foi elaborado um programa em GLIM que fornece os elementos básicos para a obtenção de árvores de divisão dicotômica hierárquica.

No caso especifico de dados com distribuição binomial este tipo de análise é preferivel àquele que utiliza, por exemplo, a transformaçăo arco seno da raiz quadrada, uma vez que um agrupamento na variável transformada pode não fazer sentido para o pesquisador que está interessado em um agrupamento na variável original. 


\section{REFERENCI AS BIBLIOGRÄFICAS}

ATKINSON, A.C. Regression diagnostics, transformations and constructed variables. Journal of the Royal Statistical Society B, Londres, 44(1): 1-36, 1982.

ATKINSON, A.C. Plots, transformations, and regression. Oxford, Clarendon Press, $1985.282 p$.

ATKINSON, A.C.; DAVISON, A.C.; NELDER, J.A. ; O'BRIEN, C.M. Model checking. Londres, Skeleton notes, Department of Mathematics, Imperial College, 1989. $115 \mathrm{p}$.

BISHOP, Y.M.M.; FIENBERG, S.E.; HOLLAND, P.W. Discrete multivariate analysis: theory and practice. Cambridge, MA., MIT Press, 1975. 557p.

BUSSAB, W. O. Hierarchical dichotomous partitions in cluster analysis. Londres, 1976. 236p. CPh.D. L.S. E. University of London).

COX,D.R. \& SPJoTVOLL,E. On partitioning means into groups. Scand 1. Statist, Noruega, 9: 147-152, 1982.

DACHS, J.N.W. Estatistica Computacional. Rio de Janeiro, 
Livros Técnicos e Cientificos Ltda, 1988. 236p.

DAVISON, A.C. Model checking 1: general regression models. Conferencia apresentada na $1 \alpha$. Escola de Modelos Lineares, São Paulo. Instituto de Matemática e Estatistica - USP, 1989 a.

DAVISON, A.C. Model checking 2 : binary data. Conferência apresentada na 1 a. Escola de modelos lineares, São Paulo, Instituto de Matemática e Estatistica - USP, $1989 \mathrm{~b}$.

FISHER, W. D. On grouping for maximum Homogeneity. Journal of the American Statistical Asssociation, Washington, 53: 789-798, 1958.

FOWLKES, E.B. Some diagnostics for binary logistic regression via smoothing. Biometrika, Londres, 74(3): $507-15,1987$.

HARTIGAN,J.A. Direct clustering of a data matrix. Journal of the American Statistical Association, Washington, 67: 123-129, 1972.

HO,L.L. Alguns aspectos da técnica AID para variável resposta categorizada. Säo Paulo, 1987. $135 \mathrm{p}$. CMestrado - Instituto de Matemática e Estatística USP).

HOFFMANN, R. \& VIEIRA, S. Análise de regressão: uma introdução à econometria. za. ed., São Paulo, 
Hucitec, 1987. 379p.

JORGENSEN, B. Maximum likelihood estimation and large-sample inference for generalized linear and nonl inear regression models. Biometrika, Londres, 70: $19-28,1983$.

KASS, G.V. Significance testing in automatic interaction detection (AID). Applied Statistics, Londres, 24: $178-89,1975$.

KASS, G.V. An exploratory technique for investigating large quantities for categorical data. Applied Statistics, Londres, 29(2): 119-27, 1980.

LEE, A.H. Assessing partial inference in generalized Iinear models. Biometrics, Alexandria, VA, 44: 71-7, 1988.

McCULLAGH, P. \& NELDER, J.A. Generalized linear models. ea. ed., Londres, Chapman and Hall, 1989. $511 \mathrm{p}$.

MORGAN, J.N. \& SONOUIST, J. A. Problems in the analysis of survey data. Journal of the American statistical Association, washington, 58: 415-434, 1963.

NELDER, J.A. \& WEDDERBURN, R.W.M. Generalized linear models. Journal of the Royal Statistical Association A, Londres, 135: 370-84, 1972.

PAYNE, G.D. (ed.) The GLIM System - release 3.77. 
164.

Oxford, Numerical Algorithms Groups Ltd, 1986. 305p.

PIERCE, D.A. \& SCHAFER, D. W. Residuals in generalized linear models. Journal of the American Statistical Association, Washington, 81: 977-86, 1986.

PREGIBON, D. Goodness of link tests for generalized linear models. Applied Statistics, Londres, 29(1): $15-24,1980$.

PREGIBON, D. Logistic Regression Diagnostics. Annals of Statistics, Hayward, CA, 9(4): 705-24, 1981.

SCOTT, A.J. \& KNOTT, M. A cluster analysis method for grouping means in the analysis of variance. Biometrics, Alexandria, 30: 507-512, 1974.

SCOTT, A. J. \& KNOTT, M. An approximate test for use with AID. Applied Statistics, Londres, 25: 103-6, 1976.

WANG, P.C. Residual plots for detecting nonlinearity in generalized linear models. Technometrics, Washington, $29(4): 435-8,1987$.

WARD Jr.,J.H. Hierarquical grouping to optimize an objetive function. Journal of the American Statistical Association, Washington, 58: 236-244, 1963.

WILLIAMS, D.A. Generalized linear model diagnostics using the deviance and single case delections. 
165.

Applied Statistical, Londres, 36(2): $181-91,1987$.

WILSON, E.B. \& HILFERTY, M.M. The distribution of chi-square. Preceedings of the National Academy of Sciences, Washington, 17: 684-8, 1931. 
166.

APENDICE 1 
O PROGRAMA DE SIMULAÇZ̃O

Para o estudo da estatistica $\Delta\left(\hat{\pi}_{d_{0}}\right)$ foi elaborado um programa de simulação escrito em linguagem GLIM 3.77 (PAYNE, 1986$).$

Considerando 10 tratamentos, 0,5 como 0 vetor de probabilidade e 20 como o numerador da binomial, a listagem a seguir é um exemplo de uma das 27 situações estudadas. 
$\backslash g \backslash$ im $\backslash$ bs im $\backslash d 9-10$.

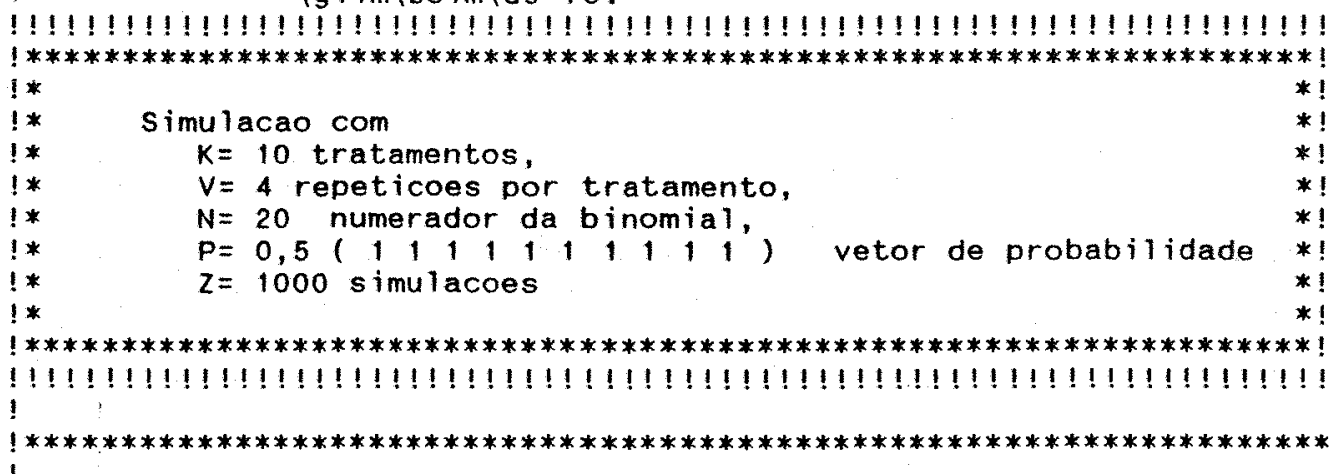

MACRO SITA simula a tabela de dados binomiais com $N=\% N$,

$K=\$ K$ tratamentos,

$V=X V$ repeticoes por tratamento

$P=$ vetor de probabilidade de comprimento $K$.

\$MACRO SITA \$DEL $* J$ Y N \$UNITS $\% X$ \$CALC $\% J=\% K$ \$IVHLE $\% J$ MI \$\$E! !

$\$ M A C R O M 1$ \$CALC $\% R=\% V$ \$WHLE $\% R$ SIMULA \$CALC $\% J=\% J-1$ \$E! $!$

I**********************************************************************

A MACRO SIMULA gera tuma amostra de uma distribuicao

binomial com $N=\mathscr{*} N$ E $P=\mathscr{} P$

\$MACRO SIMULA \$DEL G K $\% U$ WVAR $\% N K$ G \$CALC $\% I=\not N$ \$WHILE $\% I$ BIN $\$$ ! $\$ C A L C K=\% C U(G): \quad \% U=(\% J-1) * \% V+\% R: Y(\% U)=K(\not N): \% R=\% R-1 \$ \$ E !$

$\$ M A C R O$ BIN $\$ C A L C$ G( $\% I)=\% G T(P(\% J), \% S R(0)): \% I=\% I-1 \$ \$ E !$

!**********************************************************************

A MACRO SCKN fornece a analise de agrupamentos para $K$ grupos

$\$ M A C R O$ SCKN !

\$DEL A B DV RT \$VAR \%K DV A B \$VAR $\% X$ T \$YVAR YV \$ERROR B N \$!

$\$ C A L C * T=(\% C+\% F * \% S) /(\% K+\not F) \cdots \$$ sigma2 0

$\$ C A L C \quad T=2: D V(1)=\% B$ : $\% M=0: * 0=\$ K$ KWHILE $\% O$ AJU1 $\$$ !

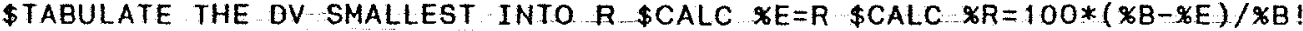

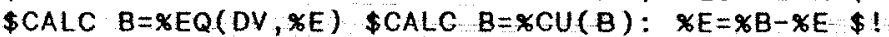

$\$ C A L C \quad \% L=\not P I /(2 *(x P I-2)) * \approx E / \% T ; \% M=x P I /(2 *(\approx P I-2)) * \% E / \% S !$

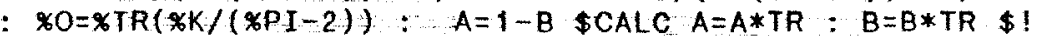

$\$ A R G$ CHIP $\approx L$ \%O \$CAL $\% Q=\# C H I P \$$ !

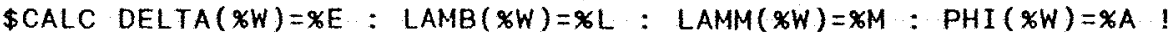

: $\operatorname{IND}(\% W)=\not W: \% W=\not W-1 \$ !$

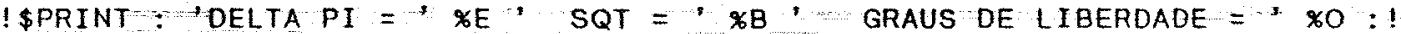

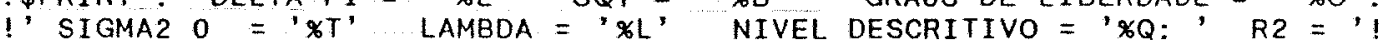
I\%R' POR CENTO'::' DIVISAO EM : ':*2 A , / '*2 B ::\$! \$TRANS \$\$E!

! **********************************************************************

\$MACRO AJU1 \$CALC $\$ L=\% \mathrm{~V}$ \$WHILE $\%$ L AJU2 \$!

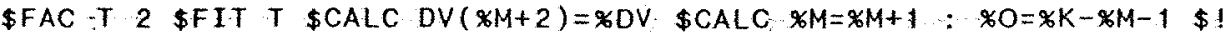
$\$ E !$

!

\$MACRO AJU2 \$CALC $T(\% L+\% M * \% V)=1 \quad \% L=\$ L-1 \quad \$ \$ E \$$

!

|********************************************************************** 
\$MACRO ANALISE :

\$CALC $N=$ KN \$ERROR B N \$YVAR $Y$ \$TRANS \$!

\$FIT \$CAL $\% B=\approx D V: \% C=\% D F$ \$FAC TRAT $\% K$ \$FIT TRAT \$!

$\$ C A L \quad \% R=(\% B-\% D V) * \% D F /(\% D V *(\% C-\% D F)): \% D=\approx C-\approx D F: \approx A=\approx \times 2 / \% D F: !$

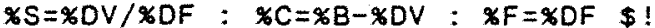

!\$PRINT : ' RAZAO DE DEVIANCES MEDIAS $=$ ' $\%$ ' $C O M{ }^{\prime} * 2 * 0$ ' ${ }^{\prime}$ '!

! $* 2$ \%DF ' G.L.':" OEVIANCE = *

!:' PARAMETRO DE DISPERSAO $=$ '\%A :' DEVIANCE MEDIA RESIDUAL $=$ ' $* S:$

$\$$ TAB THE XFV MEAN FOR TRAT INTO MED \$VAR XK TR \$!

\$CA TR=XCU(1) \$SORT TR TR MED : MED !

I\$PRINT:: 'GERMINACAO MEDIA POR TRATAMENTO'\$LOOK TR MED \$PR:!

\$SORT TRAT TRAT \%FV : YV Y XFV \$TRANS\$!

\$USE SCKN \$TRANS\$!

$\$ \$ E \$$

!**********************************************************************

\$MACRO GERA \$TRANS!

\$PRINT: : GERACAO NUMERO $=* * 4 \% \mathrm{~W} \$$

\$USE SITA $\% N$ \%K $\% V$ P \$!

\$PRINT Y \$!

\$USE ANALISE \$\$E\$

$1 * * * * * * * * * * * * * * * * * * * * * * * * * * * * * * * * * * * * * * * * * * * * * * * * * * * * * * * * * * * * * * * * * * * * * * *$

Author: R. A. Reese, Hull University, U. K.

Version 1.0 GLIM 3.77 December 1984

Main macros:

CHIP returns upper-tail chi-squared probability of $* 1$ with $\% 2$ df.

Formal arguments:

*1 The value of the chi-squared variate

\$2 The number of degrees of freedom

Macro arguments:

None

Example of use:

\$arg chip $\%$ dv $\%$ df $\$$

$\$ c a l$ \#chip\$

\$MAC CHIP !

ЖEQ $(\% 2,1) *(2-2 * \% N P(\% S Q R(\% 1)))+$ SPECIAL CASE, 1 DF.

\%EQ $(\% 2,2) *(\% E X P(-\% 1 / 2))+$ SPECIAL CASE, 2 DF.

\%GT $(\% 2,2) *(1-\% N P(((\% 1 / * 2) * *(1 / 3)-1+2 /(9 * \% 2)) / \% \operatorname{SQR}(2 /(9 * \% 2)))) !$

SENDMAC

! $* * * * * * * * * * * * * * * * * * * * * * * * * * * * * * * * * * * * * * * * * * * * * * * * * * * * * * * * * * * * * * * * * * * * * *$

$! * * * * * * * * * * * * * * * * * * * * * * * * * * * * * * * * * * * * * * * * * * * * * * * * * * * * * * * * * * * * * * * * * * * * * *$

$\$ C A L C \quad \% N=20 ; \% K=10: \% V=4 ; \% Z=1000 \%$

\$ASSIGN $P=0.5,0.5,0.5,0.5,0.5,0.5,0.5,0.5,0.5,0.5 \$ !$

\$CALC $\% X=\% K * \% V$ \$UNITS $\% X \$ !$

\$VAR $\% Z$ LAMB LAMM DELTA D3 PHI IND IDE IDD SUF \$!

\$TRANS\$!

$\$ C A L C$ TRAT $=\% G L(\% K, \approx V): R E P=\approx G L(\% V, 1) \$ !$

$\$ C A L C \quad \% W=\% Z$ \$WHILE $\% W$ GERA $\$ !$

\$TRANS W H O F \$!

\$PRINT :: $\quad * * * * *$ detta $* * * * * 3: \$ 1$

\$SORT DELTA \$!

\$TABULATE THE DELTA MEAN INTO MD : THE OELTA VARIANCE INTO VD \$!

\$CALC \%R = VD/MD \$AC 4\$!

\$PRINT:" MEDIA DE DELTA = 'MD'; VARIANCIA OE DELTA = "VD !

:' ; RAZAO = 'GR: \$!

\$HIST DELTA ' $*$ ' \$PRINT; HISTOGRAMA DE DELTA' : \$! 


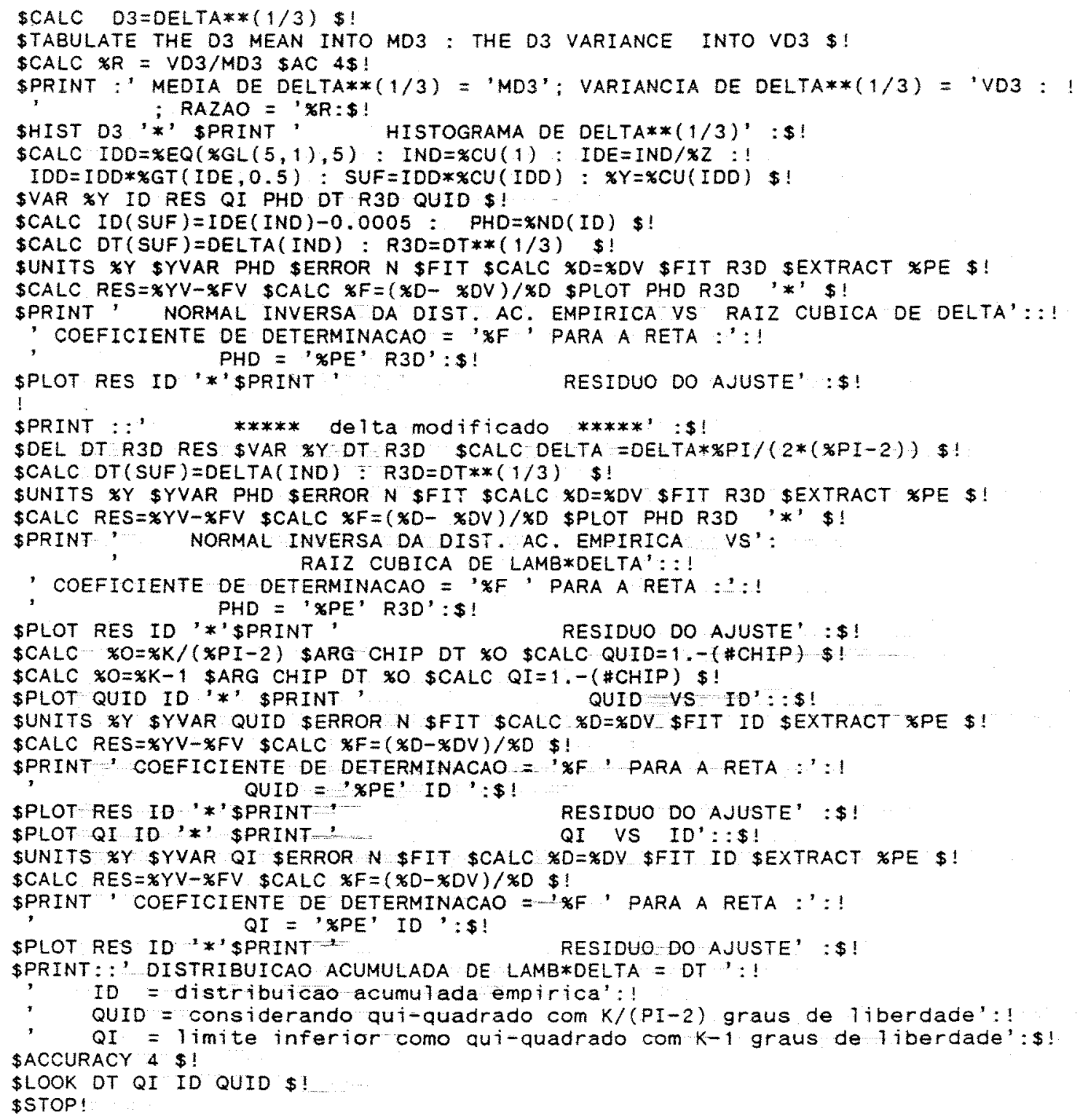


APENDICE 2 


\section{RESULTADO DE UMA DAS SIMULAÇOES}

- programa de simulação exibido no Apêndice 1 foi rodado em 27 situações considerando as variações do número de tratamentos $(3,6,10)$, as variaçōes do numerador da binomial $(10,20,50)$ e as variaçós da probabilidade da binomial $c 0,10 ; 0,30$; 0,50 ).

O equipamento utilizado foi um PC AT 486-DX de $50 \mathrm{Mhz}$ com $4 \mathrm{Mb}$ de memória RAM que executou o programa de simul açăo em 9 tempos medios diferentes, uma vez que a mudança da probabilidade da binomial não produz nenhuma al ter ação no tempo de execução ( Tabela AZ.1).

Tabela A2.1 - Tempo médio de execução do programa de simul ação considerando diferentes valores de $K$ e $N$

\begin{tabular}{cccc}
\hline$N \backslash K$ & 3 & 6 & 10 \\
\hline 10 & $00: 09: 25$ & $00: 18: 25$ & $00: 31: 22$ \\
20 & $00: 13: 17$ & $00: 25: 38$ & $00: 44: 00$ \\
50 & $00: 24: 53$ & $00: 48: 45$ & $01: 22: 00$ \\
\hline
\end{tabular}

A seguir está o resultado da simulação, obtido considerando-se 10 tratamentos, denominador da binomial igual à 50 e a probabilidade igual à 0,1 , executado em aproximadamente em $1 \mathrm{~h}$ e $22 \mathrm{~min}$. 
lof GLIM 3.77 update 1 (copyright) 1985 Royal Statistical society, Lonoon

[0]

[i] ? $\sin 11$ s

[i] File name? Iglim

[i] !

$\$ g]$ im\$bsim\$d9-10

[i] !!!!!!!!!!!!!!!!!!!!!!!!!!!!!!!!!!!!!!!!!!!!!!!!!!!!!!!!!!!!!!!!!!!!!!!!!!!!

$[i] ! * * * * * * * * * * * * * * * * * * * * * * * * * * * * * * * * * * * * * * * * * * * * * * * * * * * * * * * * * * * * * * * * * * * !$

[i] $! *$

[i] !*

[i] $! *$

[i] $! *$

[i] !*

[i] !*

[i] !*

[i] !*

Simulacao com

$K=10$ tratamentos,

$V=4$ repeticoes por tratamento,

$N=50$ numerador da binomial,

$z=1000$ simulacoes

vetor de probabilidade

[1] $[* * * * * * * * * * * * * * * * * * * * * * * * * * * * * * * * * * * * * * * * * * * * * * * * * * * * * * * * * * * * * * * * * * * 1$

[i] !!!!!!!!!!!!!!!!!!!!!!!!!!!!!!!!!!!!!!!!!!!!!!!!!!!!!!!!!!!!!!!!!!!!!!!!

[i] !

[i] $: * * x+$

[i]!

[i] :

[i] !

[i] :

[i] :

MACRO SITA simula a tabela de dados binomiais com $N=x N$,

$K=x K$ tratamentos,

$V=2 V$ repeticoes por tratamento

[i] :

[i] $!$

[j] \$MACRO SITA \$DEL $\% J$ Y $N$ \$UNITS $\% X$ \$CALC $\$ J=\% K$ \$WILE $\% J$ MI \$\$E!

[i] !

[i] \$MACRO M1 \$CALC $\% R=\% V$ \$WHLE $\% R$ SIMULA \$CALC $\% J=\% J-1 \$ \$ E$ !

[i] !

[j] $! * * * * * * * * * * * * * * * * * * * * * * * * * * * * * * * * * * * * * * * * * * * * * * * * * * * * * * * * * * * * * * * * * * * * * *$

[i] 1

[i] 1

[i] !

A MACRO SIMULA gera uma amostra de uma distribuicao

binomial com $N=\mathscr{K}$. E $P=\% P$

[i] !

[i] \$MACRO SIMULA \$DEL GK $\% U$ \$VAR $\% N K$ G \$CALC $\% I=X N$ \$WHILE $\% I$ BIN \$!

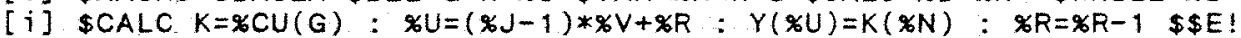

[i] !

[i] \$MACRO BIN \$CALC $G(\% I)=\$ G T(P(\% J), \% S R(0)): x I=\% I-1 \$ \$ E$ !

[i] !

[i] $[* * * * * * * * * * * * * * * * * * * * * * * * * * * * * * * * * * * * * * * * * * * * * * * * * * * * * * * * * * * * * * * * * * * * * * \pi$

[i] !

[i] 1

[i] !

[i] \$MACRO SCKN !

[i] \$DEL A B DV R T \$VAR \%K DV A B \$VAR $\% X$ T \$YVAR. YV \$ERROR B N \$!

[i] \$CALC $\% \mathrm{~T}=(\% \mathrm{C}+\% F * \% S) /(\% \mathrm{~K}+\% \mathrm{~F}) \ldots$ \$ sigma2 0

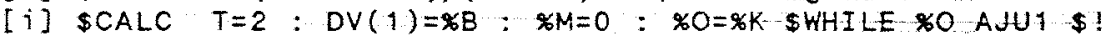

[i] \$TABULATE THE DV SMALLEST INTO R \$CALC $\$ E=R \quad \$ C A L C \% R=100 *(x B-\% E) / \% B$

[i] $\$ C A L C B=\% E Q(D V, \% E)$ \$CALC $B=\% C U(B) ; \% E=\% B-\% E \$ 1$

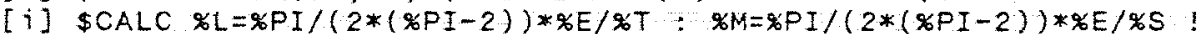

[i] $: \% O=\$ T R(\$ K /(\$ P I-2)): A=1-B$ \$CALC $A=A * T R: B=B * T R \$$ !

[i] \$ARG CHIP $\%$ L $\% O$ \$CAL $\% Q=\# C H I P$ \$!

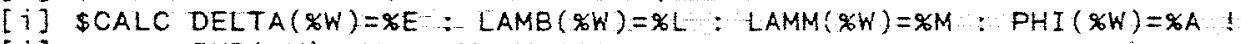

[j] : $\operatorname{IND}(\mathscr{W W})=\$ W: \% W=\not W-1 \$$ !

[i] !\$PRINT: 'DELTA PI $=$ ' $x E$; SQT $=x B$ ' GRAUS DE LIBERDADE = $\% O:$

[i] !' SIGMA2 $0=$ ' $\%$ ' LAMBDA =' $\%$ ' NIVEL DESCRITIVO =' $\% Q:$ : R2 $=$ '!

[i] $\%$ ' ' POR CENTO'::' DIVISAO EM : ': $* 2 \mathrm{~A}, /, * 2 \mathrm{~B}:: \$$ !

[j] \$TRANS \$\$E!

[i] !

$[j] 1 * * * * * * * * * * * * * * * * * * * * * * * * * * * * * * * * * * * * * * * * * * * * * * * * * * * * * * * * * * * * * * * * * * * * * *$

[i]

[i] \$MACRO AJU1 \$CALC $\approx L=X V$ \$WHILE $\otimes L$ AJU2 \$!

[i] $\$ F A C T 2$ \$FIT T $\$ C A L C$ DV $(\$ M+2)=\$ D V$ SCALC $\$ N_{i}=x M+1: x O=x K-\not M-9 \$$

[i] $\$ E$ 


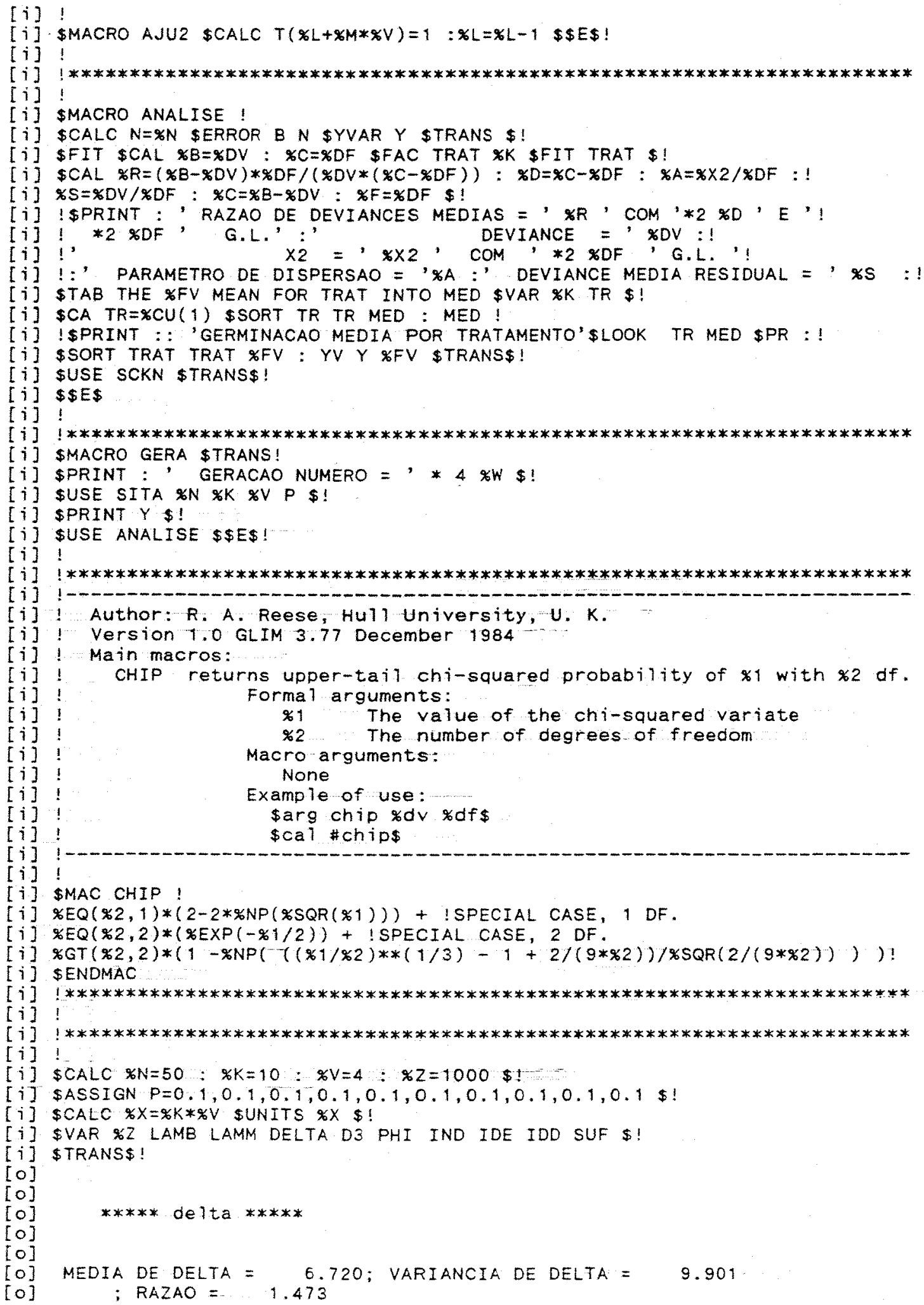




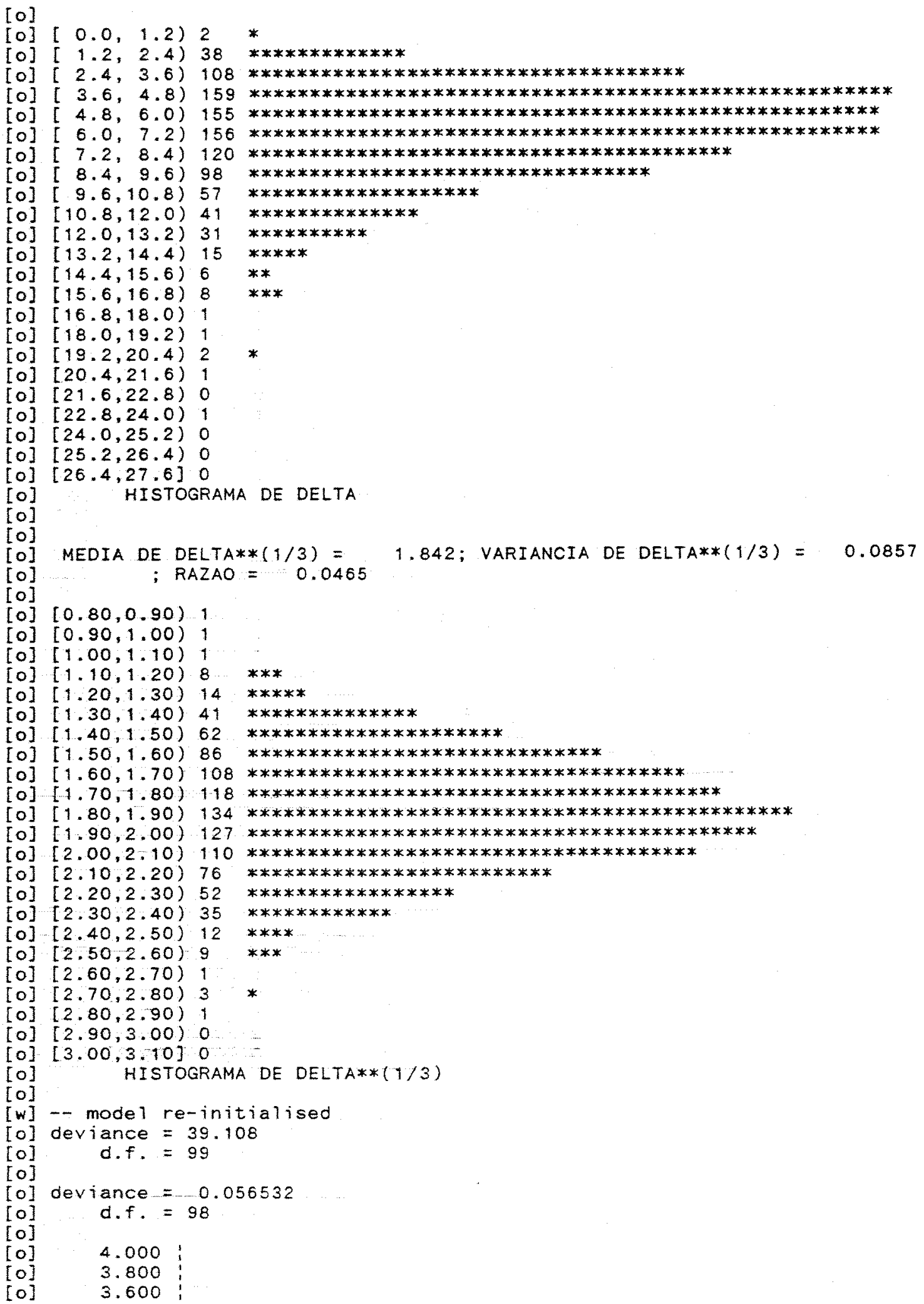




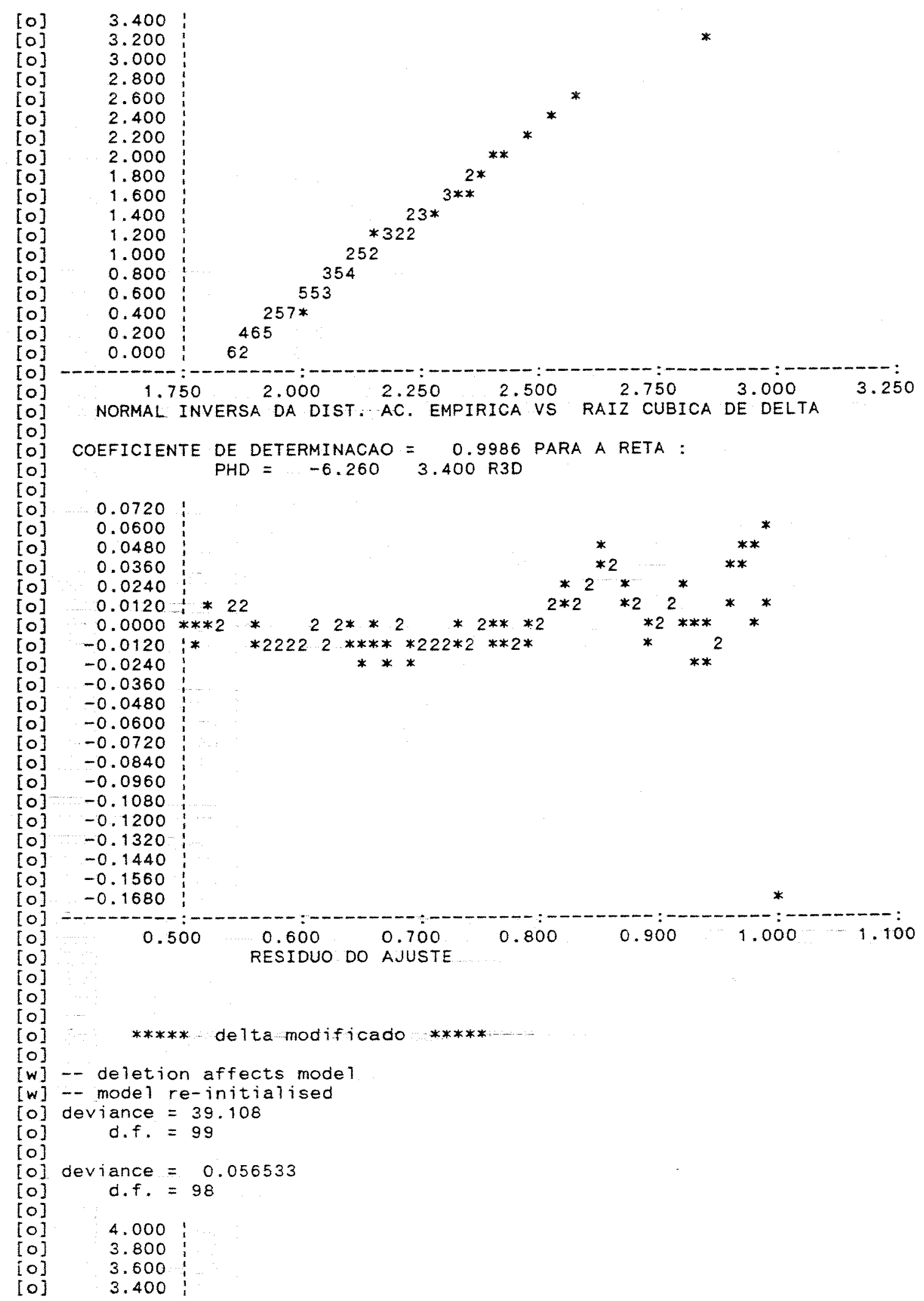




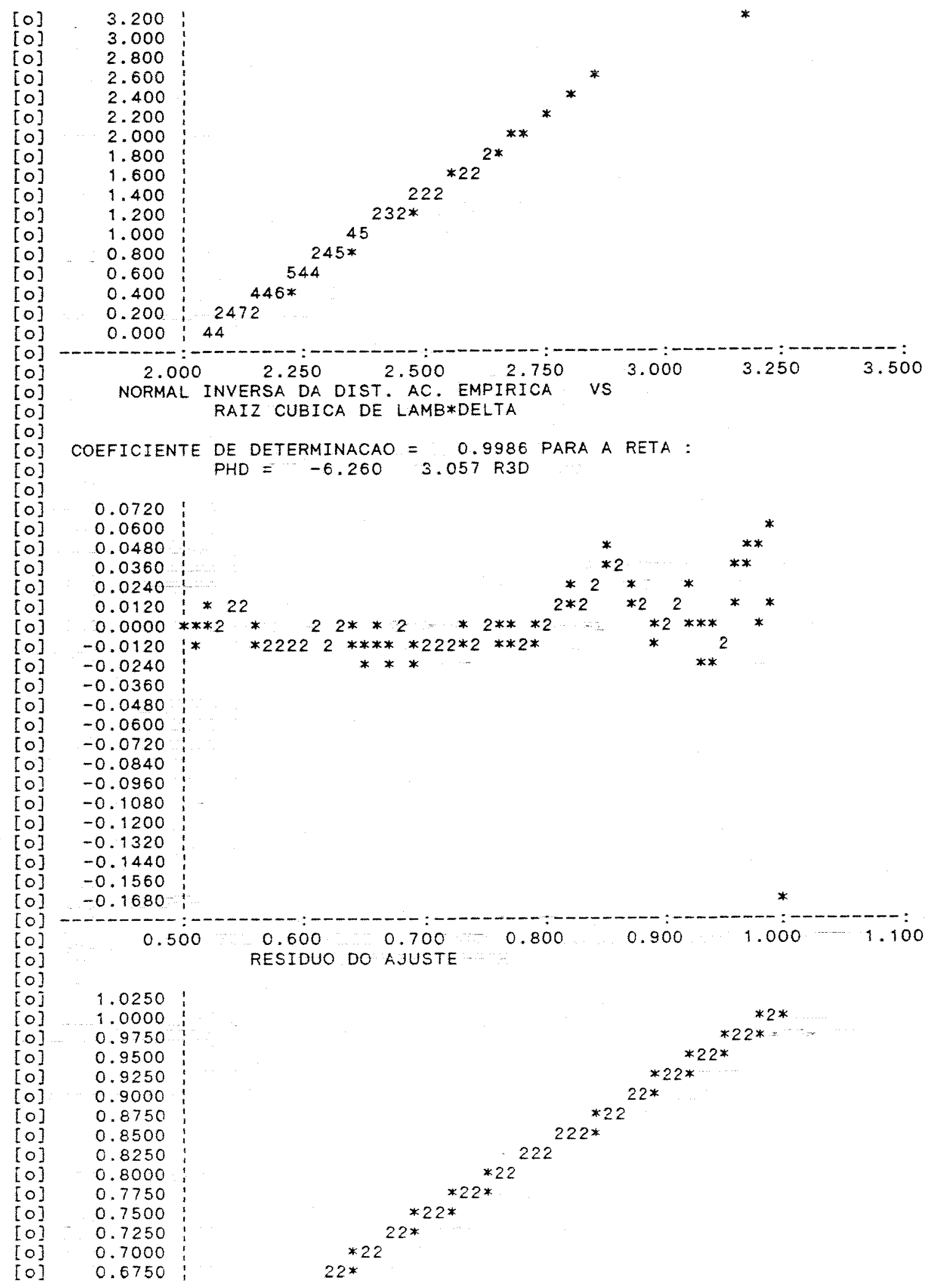




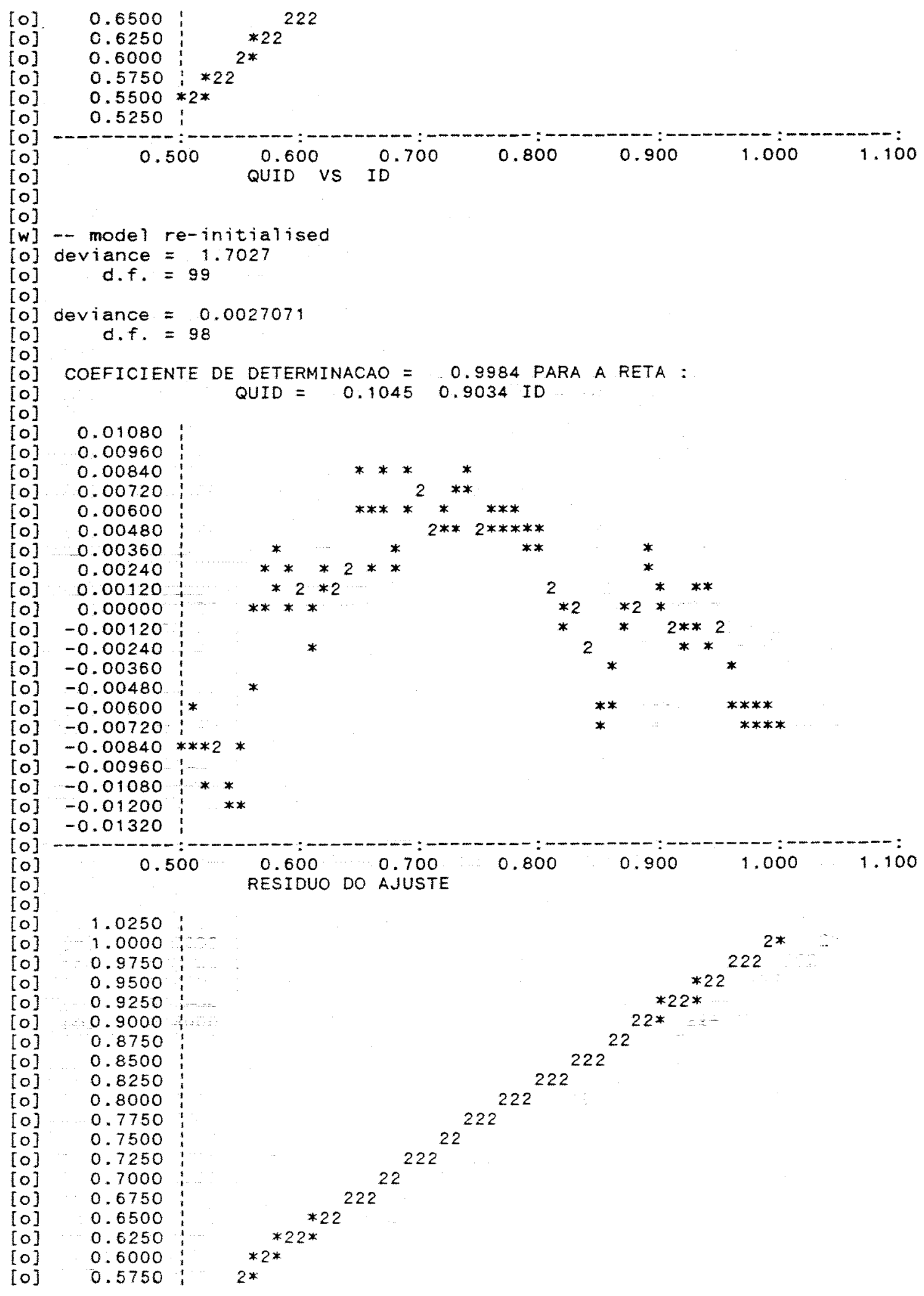




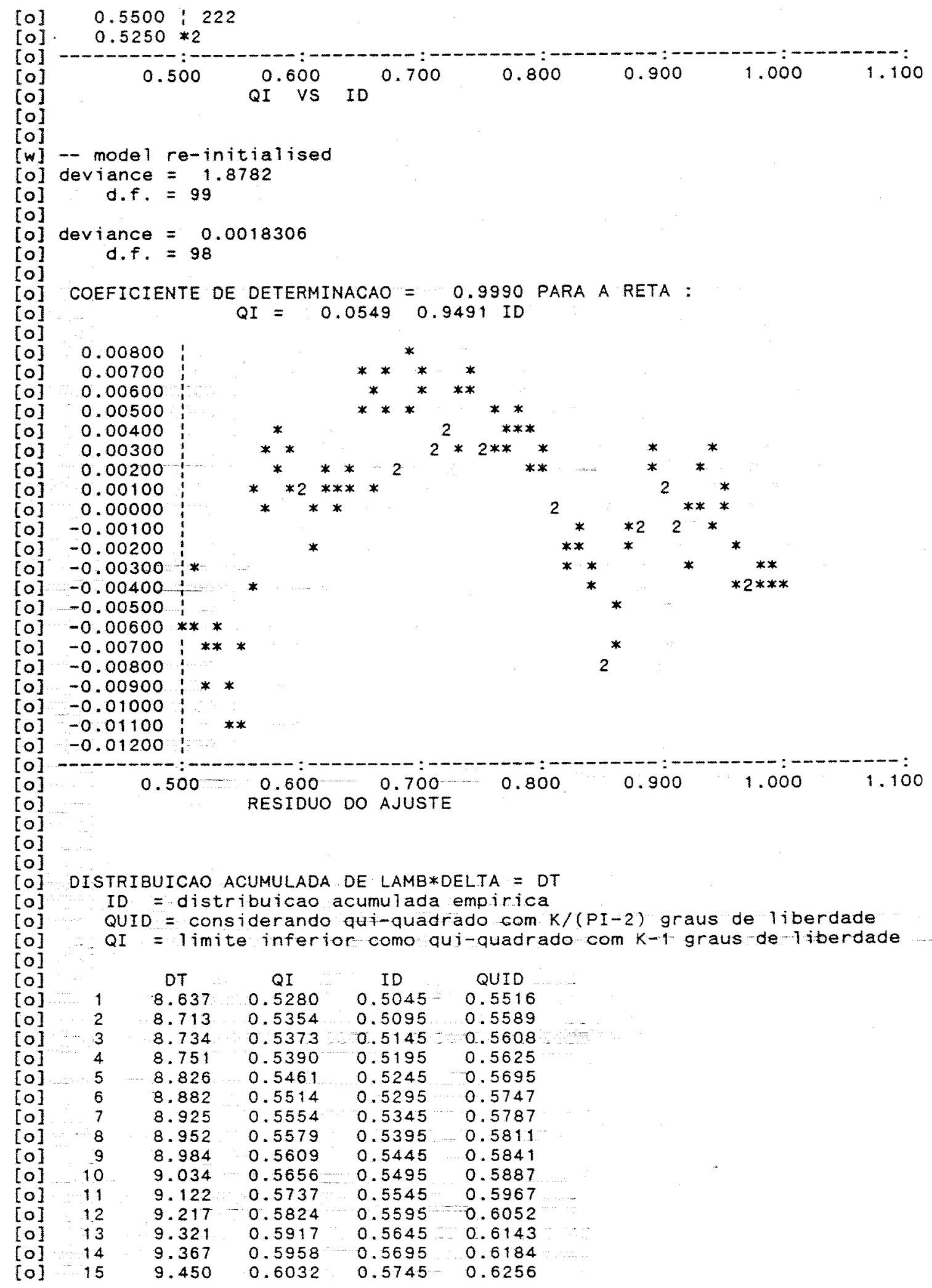




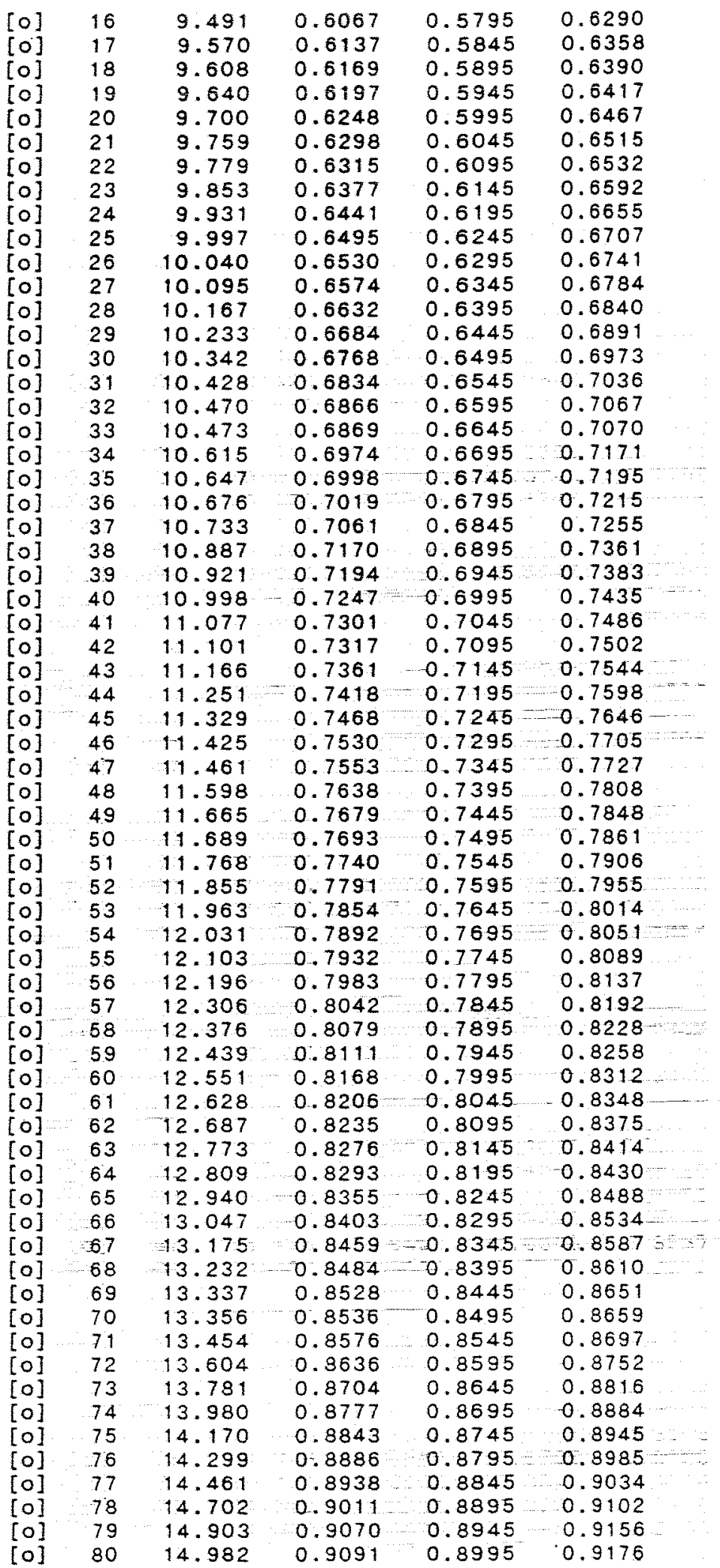


181.

$\begin{array}{lrrlll}{[0]} & 81 & 15.185 & 0.9145 & 0.9045 & 0.9226 \\ {[0]} & 82 & 15.291 & 0.9173 & 0.9095 & 0.9251 \\ {[0]} & 83 & 15.472 & 0.9217 & 0.9145 & 0.9292 \\ {[0]} & 84 & 15.609 & 0.9250 & 0.9195 & 0.9322 \\ {[0]} & 85 & 15.927 & 0.9320 & 0.9245 & 0.9387 \\ {[0]} & 86 & 16.160 & 0.9368 & 0.9295 & 0.9431 \\ {[0]} & 87 & 16.556 & 0.9442 & 0.9345 & 0.9499 \\ {[0]} & 88 & 16.854 & 0.9492 & 0.9395 & 0.9545 \\ {[0]} & 89 & 16.946 & 0.9507 & 0.9445 & 0.9558 \\ {[0]} & 90 & 17.361 & 0.9569 & 0.9495 & 0.9614 \\ {[0]} & 91 & 17.669 & 0.9610 & 0.9545 & 0.9651 \\ {[0]} & 92 & 17.881 & 0.9636 & 0.9595 & 0.9675 \\ {[0]} & 93 & 18.133 & 0.9664 & 0.9645 & 0.9701 \\ {[0]} & 94 & 18.608 & 0.9713 & 0.9695 & 0.9745 \\ {[0]} & 95 & 19.070 & 0.9754 & 0.9745 & 0.9782 \\ {[0]} & 96 & 19.719 & 0.9802 & 0.9795 & 0.9825 \\ {[0]} & 97 & 20.831 & 0.9865 & 0.9845 & 0.9881 \\ {[0]} & 98 & 21.912 & 0.9907 & 0.9895 & 0.9919 \\ {[0]} & 99 & 23.372 & 0.9945 & 0.9945 & 0.9952 \\ {[0]} & 100 & 32.110 & 0.9998 & 0.9995 & 0.9998\end{array}$


182.

APÉNDICE 3 
183.

LISTAGENS DO PROGRAMA E DA EXECUÇÃO DO PROGRAMA DE ANÁLISE DE AGRUPAMENTOS EM LINGUAGEM GLIM

A seguir encontram-se as listagens do programa de análise de agrupamento e de sua execução considerando três tipos de modelo: logito, probito e transformação arco seno da raiz quadrada da proporção.

As MACROS usadas são basicamente as mesmas do Apéndice 1. Optou-se pelo cálculo de todos os ramos possiveis para a construção da arvore de divisão di cotómica. 
\glimlclusterlproge.

!!!!!!!!!!!!!!!!!!!!!!!!!!!!!!!!!!!!!!!!!!!!!!!!!!!!!!!!!!!!!!!!!!!!!!!!!!!!

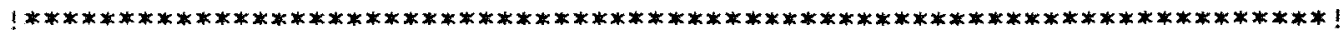

\section{\$TRANS}

\$DEL M A B DV R T \$VAR $\% K$ DV A B \$YVAR YV \$!

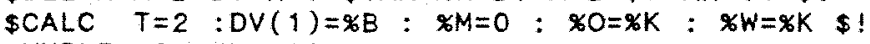

\$WHILE $\% O$ AJU1 \$!

\$TABULATE THE DV SMALLEST INTO R \$?

$\$ C A L C \times E=R: \% R=100 *(\% B-\% E) / \% B$ ! calcula $r 2$ dessa divisao

$\$ C A L C B=\% E Q(D V, \% E)$ \$CALC $B=\% C U(B): x E=\% B-x E \$ ! \quad \% E=$ delta

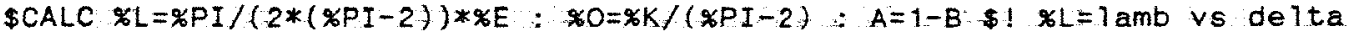
\$ARG CHIP $\% L \% O$ \$CALC $\% Q=\# C H I P \$ !$

\$CALC A=A*TR : $B=B * T R$ \$TRANS $O \$$ !

$\$$ PRINT :' DELTA $O=$ ' $\%$ ' DV $(1)=$ ' $\% B$ ' GRAUS DE LIBERDADE = ' $\% O$ !

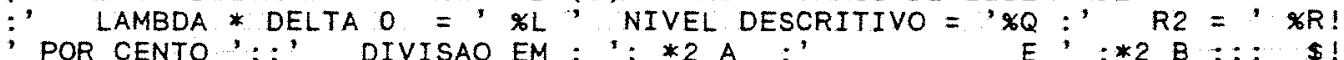
\$E!

ENTRADA : $: * 1$ = codigo dos tratamentos ja ordenados pela media

\%2 = vetor de observacoes dos tratamentos de interesse ja ordenado pela media

\%3 = numero de grupos

\section{\$MACRO SKB2}

STRANS

$\$ D E L M A B$ DV R T \$CALC $\% W=\$ 3: \% X=\$ V * \% W$ SUNITS $\% X \$$ !

\$CALC $M=100$ \$YVAR $\% 2$ \$VAR $\% W$ DV A B \$ERROR B M I

\$FIT \$CALC $\% G=$ WOV ? . soma de quadrados total dentro do grupo

\$CALC $T=2 \quad$ DV $(1)=\% G: \% H=0: \% O=\% W$ !

\$WILE $\approx O$ AJU1 \$!

\$TABULATE THE DV SMALLEST INTO R \$CALC $¥ E=R \$$ !

$\$ C A L C$ OR $=100 *(\% G-\% E) / \% B$ ! calcula $r 2$ dessa divisao

$\$ C A L C B=x E Q(D V, \approx E) \quad \$ C A L C B=x C U(B): \% E=x G-x E \$$ !

$\$ C A L C \quad x L=\$ P I /(2 *(\% P I-2)) * \% E ; \quad x C=\$ W /(\% P I-2) \cdots A=1-B ; \$ 1$

\$ARG CHIP $\% L$ \%O \$CALC $\% Q=\#$ CHIP $\$ !$

$\$ C A L C A=A * \$ 1: B=B * \% 1$ \$TRANS $0 \%$ \$

\$PRINT: $:$ DELTA $0=$ ' $\%$ DV $(1)=' \% B^{\prime}$ GRAUS OE LIBERDADE $=$ ' $\% O !$

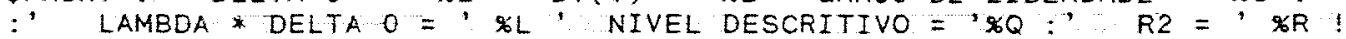




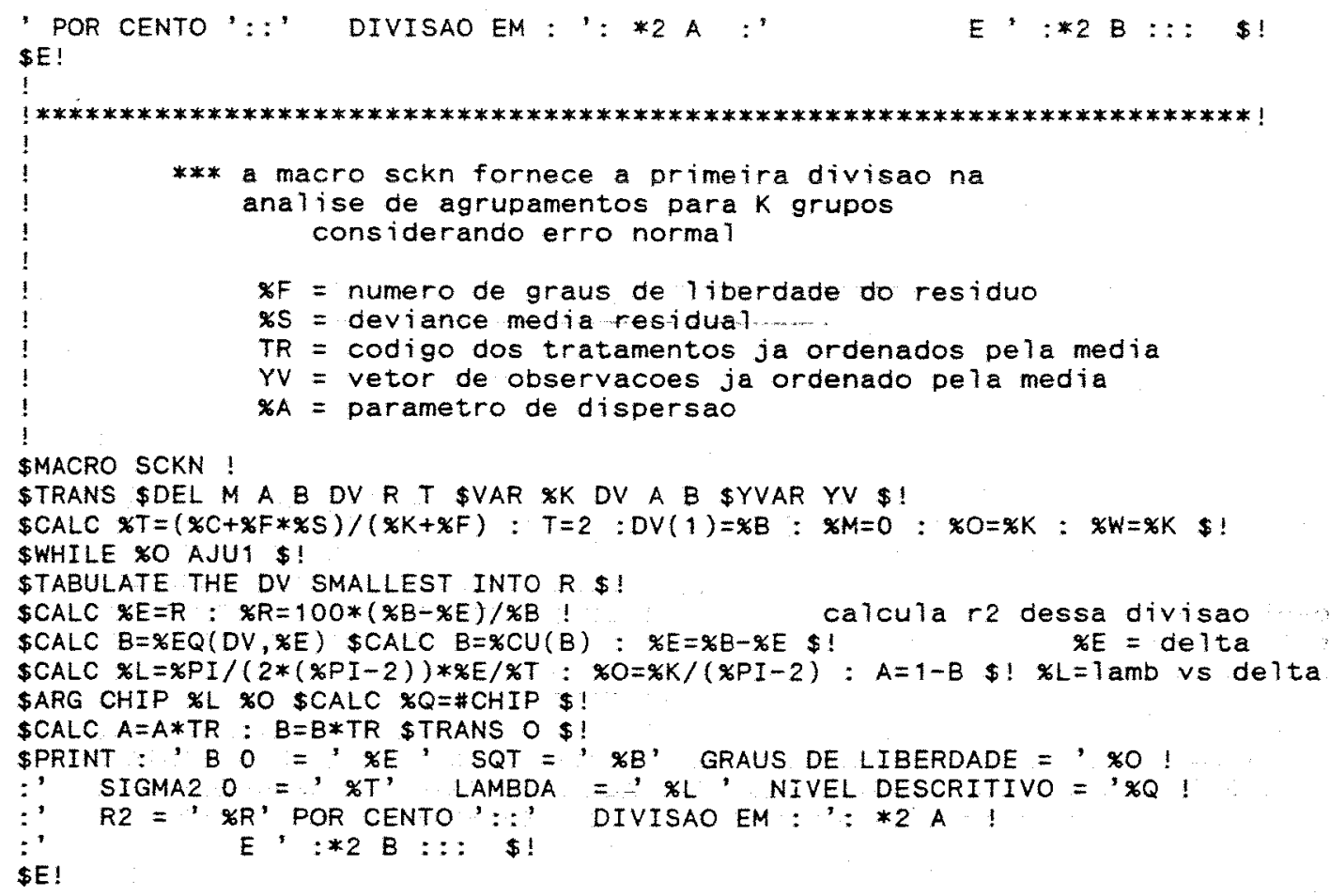


Author: R. A. Reese, Hull University, U. K.

version 1.0 GLIM 3.77 December 1984

Main macros:

CHIP returns upper-tail chi-squared probability of $* 1$ with $\% 2$ df.

Formal arguments:

*1 The value of the chi-squared variate

\$2 The number of degrees of freedom

Macro arguments:

None-

Example of use:

$\$ a r g$ chip $x d v$ xdf $\$$

$\$ c a 1$ \#chip\$

I \$MAC CHIP !

! $\%$ LT $(\% 2,2) * \% E Q(* 2,1) *(2-2 * \approx N P(* S Q R(* 1)))+1$ SPECIAL CASE, 1 DF.

!\%LT\%EQ $(\% 2,2) *(\%$ EXP $(-\approx 1 / 2))+$ ISPECIAL CASE, 2 DF.

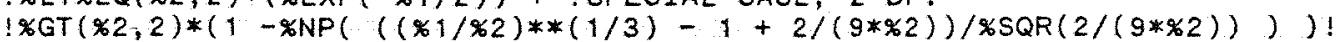

! \$ENDMAC

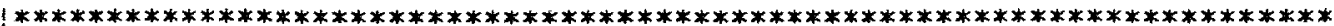

*** a macro CHIl calcula a distribuicao acumulada de

uma variavel qui-quadrado $\%$ xom $\% 0$ graus de liberdade,

usando a aproximacao de wilson e Hilferty (1931), (ver

Dachs, 1988, pg 143)-P[X2 $(\% 2)=\langle \% 1]$

\$MACRO CHIP !

\$P $=1-$ XNP $(((\$ 1 / \% 2) * *(1 / 3)-1+2 /(9 * 22)) * \% \operatorname{SQRT}(9 * \% 2 / 2)) !$

$\$ E$ !

!

!**********************************************************************

*** a macro aju1 calcula as funcoes desvio para todas

as particoes dicotomicas de medias ordenadas

\$MACRO AJU1 :

$\$ C A L C \% L=\% V$ \$WHLE $\% L$ AJU2 \$FAC T 2 \$FIT T \$!

$\$ C A L C \quad D V(\% M+2)=x D V \quad \$ C A L C \quad \% M=x M+1: \% O=\% W-x M-1 \quad \$ !$ $\$ E$ !

1

! $\star * * * * * * * * * * * * * * * * * * * * * * * * * * * * * * * * * * * * * * * * * * * * * * * * * * * * * * * * * * * * * * * * * * * * * *$

!

*** a macro aju2 calcula os vetores $T$ para a macro ajul!

\$MACRO AJU2 \$CALC $T(x L+\% M * \% V)=1: \approx L=\% L-1 \$$ !

$\$ E$ !

1

$1 * * * * * * * * * * * * * * * * * * * * * * * * * * * * * * * * * * * * * * * * * * * * * * * * * * * * * * * * * * * * * * * * * * * * * *$

** a macro tame ordena as medias dos tratamentos

\$MACRO TAME?

\$TABULATE THE \&FV MEAN FOR TRAT INTO MED \$!

\$VARIATE $\%$ K TR \$CALC TR $=\% C U(1)$ \$SORT TR TR MED \$SORT MED \$!

\$PRINT: 'MEDIAS ORDENADAS DE TRATAMENTOS '\$LOOK TR MED \$PRINT;:\$1

\$SORT TRAT TRAT KFV : YV Y \&FV \$!

$\$ \$ E$ !

!

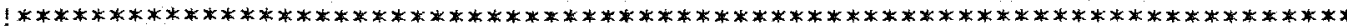

* * a macro analise calcula os elementos necessarios

para a analise de variancia

\$MACRO ANALISE \$! 


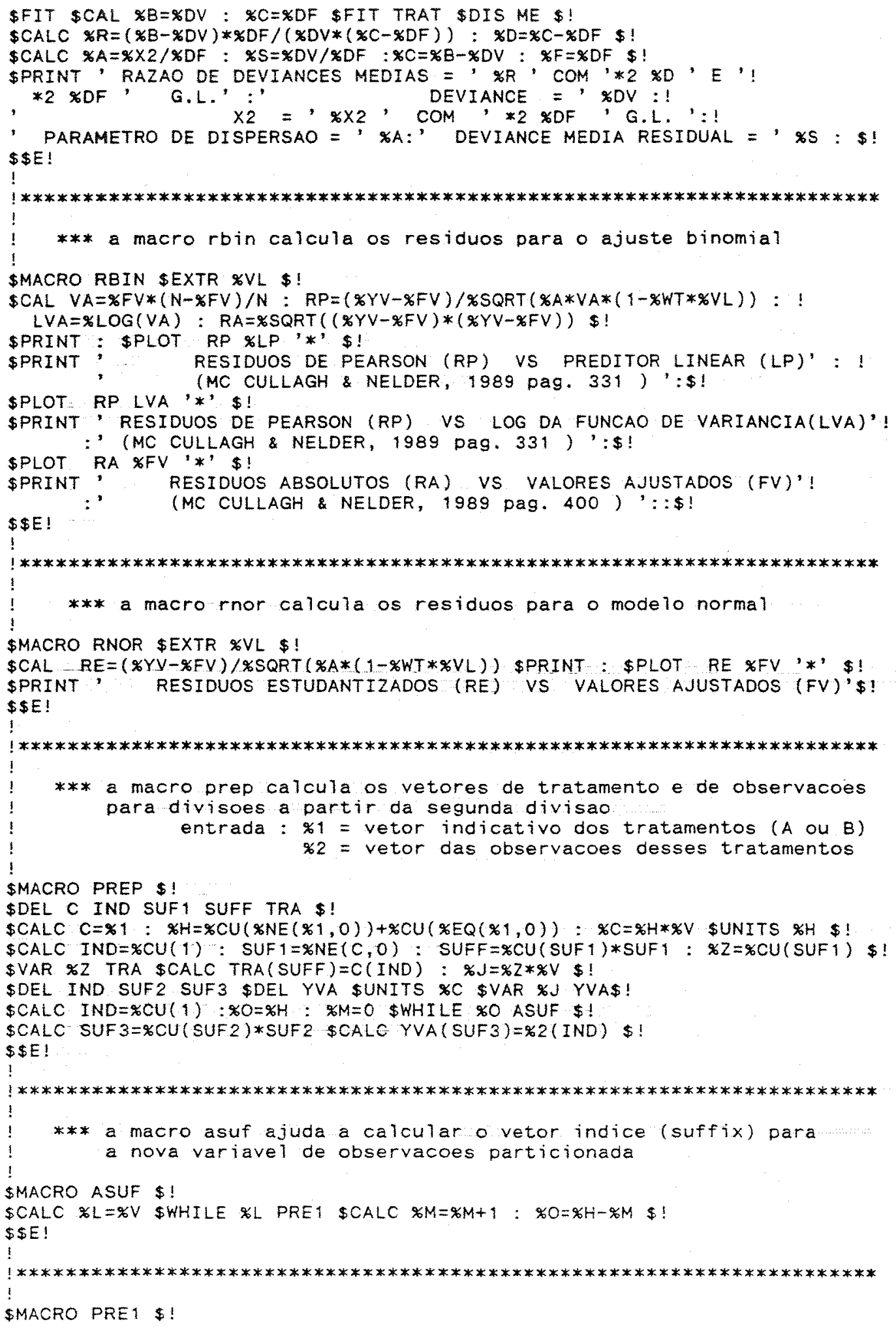




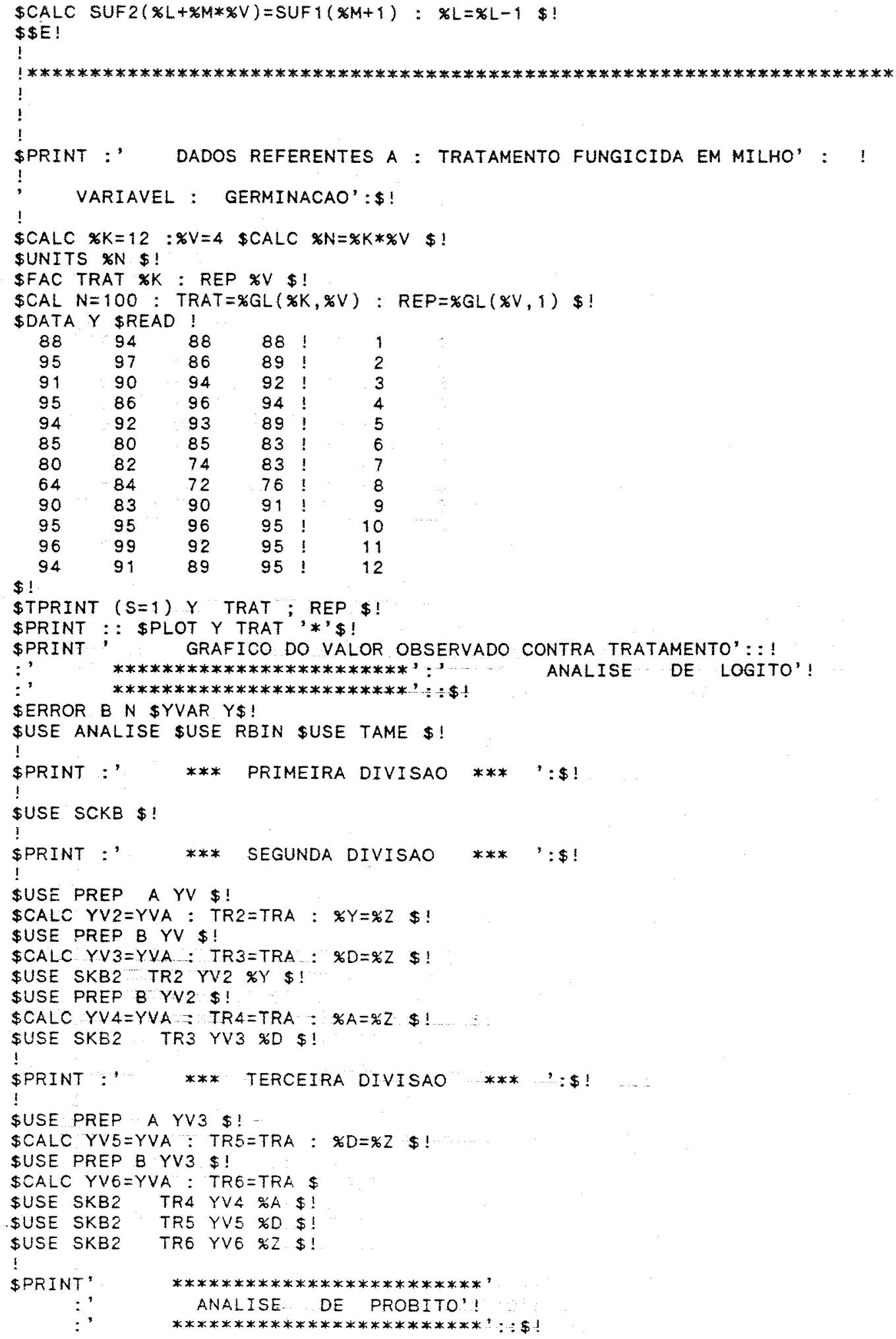


189.

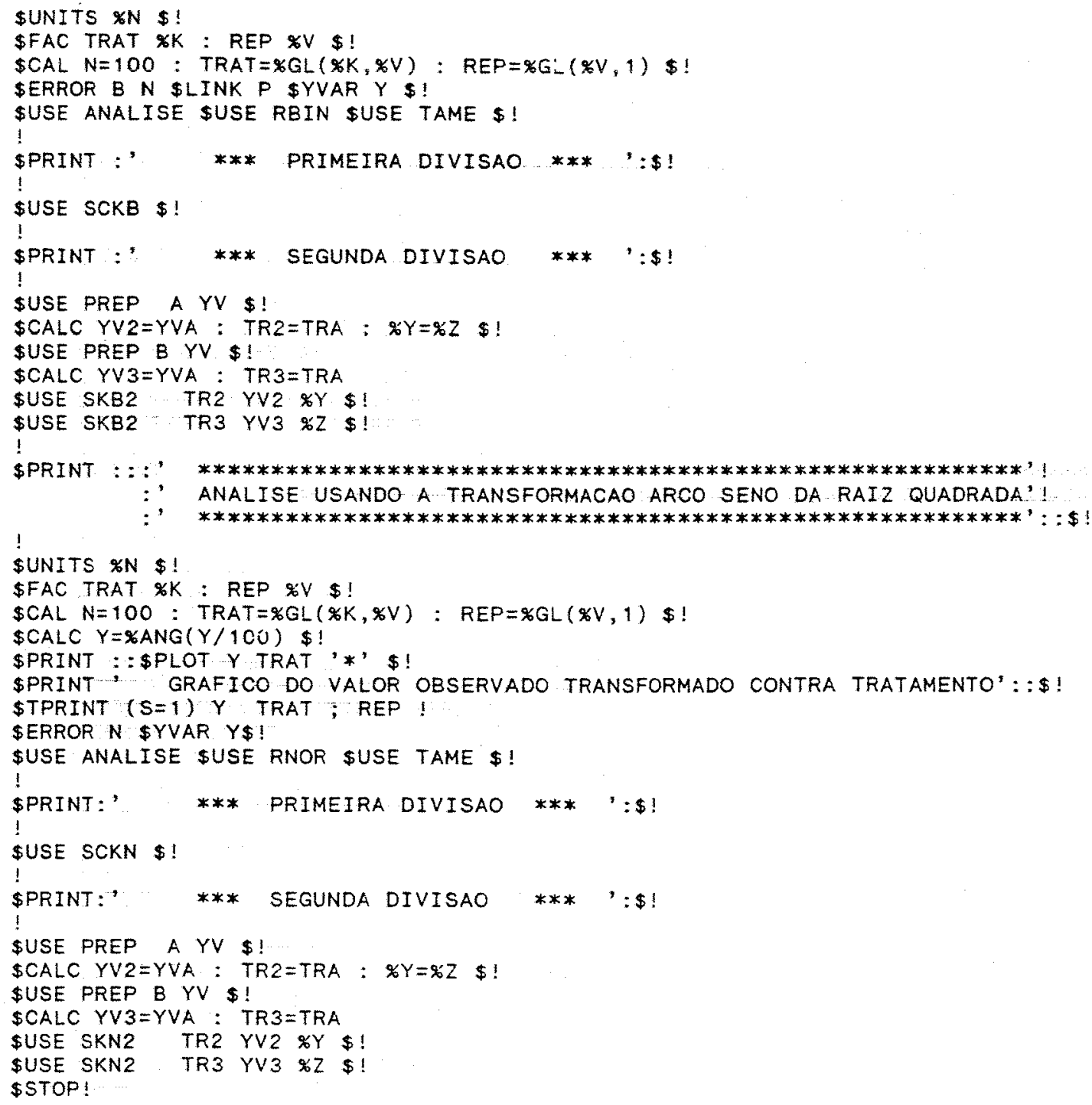


[0] GLIM 3.77 update 1 (copyright) 1985 Royal Statistical Society, London

[0]

[i] ? SIN 11 \$

[i] File name? IGLIMICLUSTER\PROG6

[i]!

$\$ 91$ im\$cluster\$prog6.

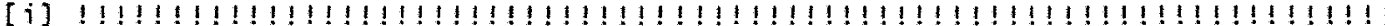

$[1] ! * * * * * * * * * * * * * * * * * * * * * * * * * * * * * * * * * * * * * * * * * * * * * * * * * * * * * * * * * * * * * * * * * * * * * !)$

[i] $! *$

[i] $! *$

ANALISE DE AGRUPAMENTO DE TRATAMENTOS

[门] !*********************************************************************1

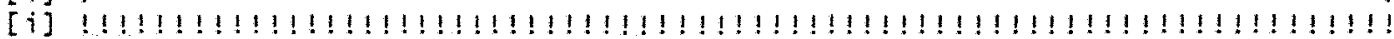

[i] !

[i] !

[i] \$TRANS O \$!

[0]

[0] : DADOS REFERENTES A : TRATAMENTO FUNGICIDA EM MILHO

[0] VARIAVEL: GERMINACAO

[0]

[0]

[0]

[0]

[0]

[0]

[0]

[0]

[0]

[0]

[0]

\begin{tabular}{|c|c|c|c|c|}
\hline $\begin{array}{l}\text { REP } \\
\text { RAT }\end{array}$ & 1 & 2 & 3 & 4 \\
\hline $\begin{array}{r}1 \\
2 \\
3 \\
4 \\
5 \\
6 \\
7 \\
8 \\
9 \\
10 \\
11 \\
12\end{array}$ & $\begin{array}{l}88.00 \\
95.00 \\
91.00 \\
95.00 \\
94.00 \\
85.00 \\
80.00 \\
64.00 \\
90.00 \\
95.00 \\
96.00 \\
94.00\end{array}$ & $\begin{array}{l}94.00 \\
97.00 \\
90.00 \\
86.00 \\
92.00 \\
80.00 \\
82.00 \\
84.00 \\
83.00 \\
95.00 \\
99.00 \\
91.00\end{array}$ & $\begin{array}{l}88.00 \\
86.00 \\
94.00 \\
96.00 \\
93.00 \\
85.00 \\
74.00 \\
72.00 \\
90.00 \\
96.00 \\
92.00 \\
89.00\end{array}$ & $\begin{array}{l}88.00 \\
89.00 \\
92.00 \\
94.00 \\
89.00 \\
83.00 \\
83.00 \\
76.00 \\
91.00 \\
95.00 \\
95.00 \\
95.00\end{array}$ \\
\hline
\end{tabular}

[o]

[0]

[0]

[0]

[0]

[0]

[0]

[0]

[0]

[0]

[0]

[0]

[0]

[0]

[0]

[0]

[0]

[0]

[0]

[0]

[o]

[0]

[0]

[0]

[0]

102.00

100.00

98.00

96.00

94.00

92.00

90.00

88.00

86.00

84.00

82.00

80.00

78.00

76.00

74.00

72.00

70.00

68.00

66.00

64.00

[0]

0.00

GRAFICO DO VALOR OBSERVADO CONTRA TRATAMENTO

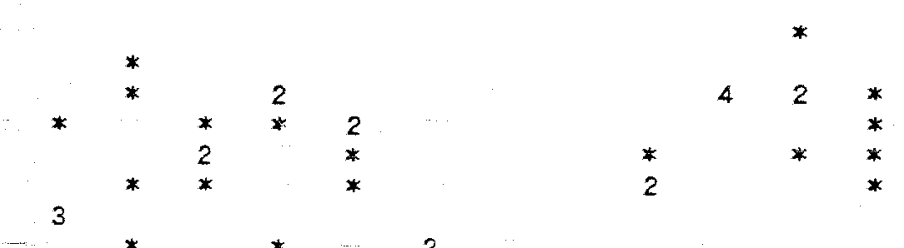

[0] 


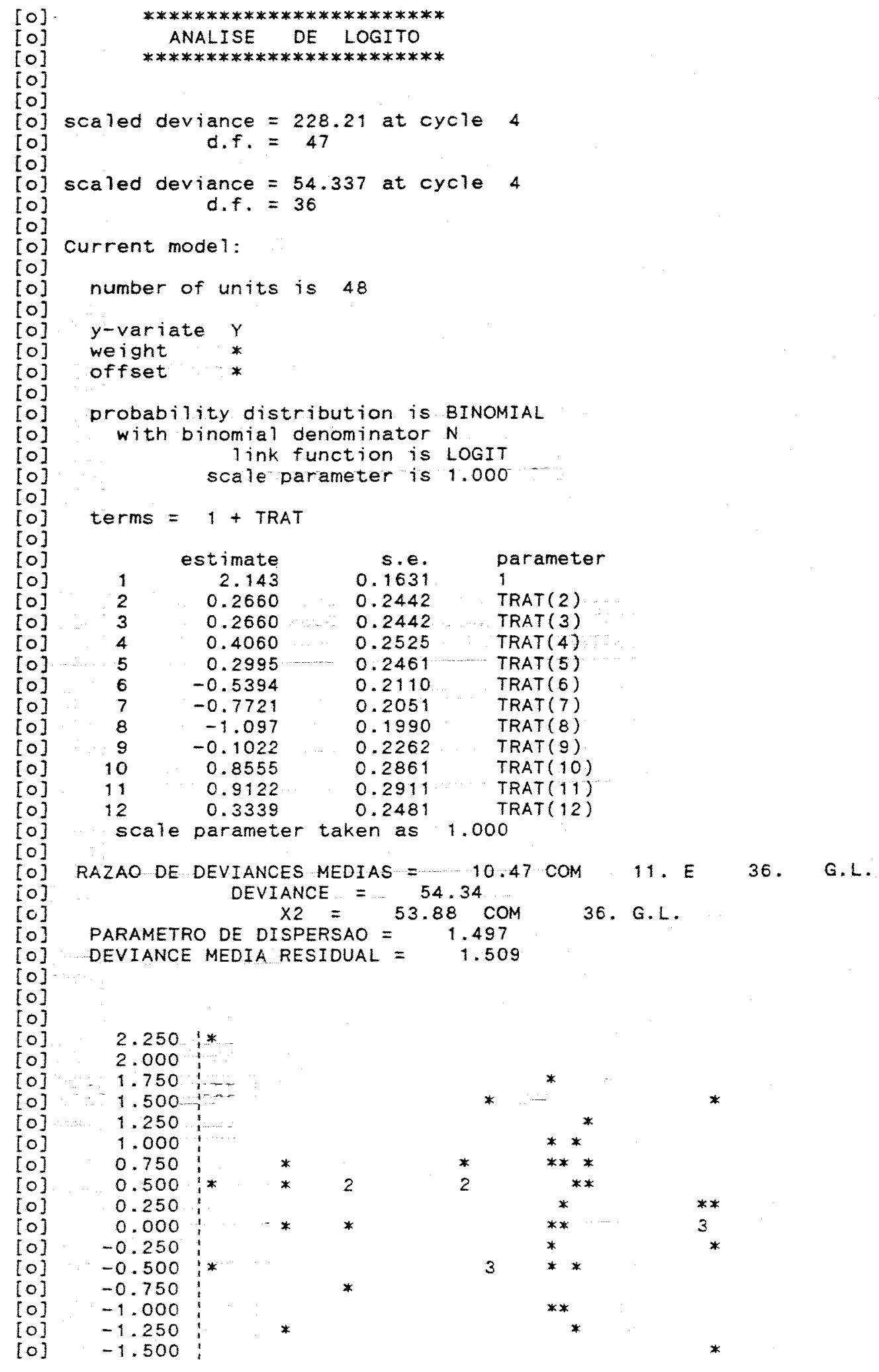




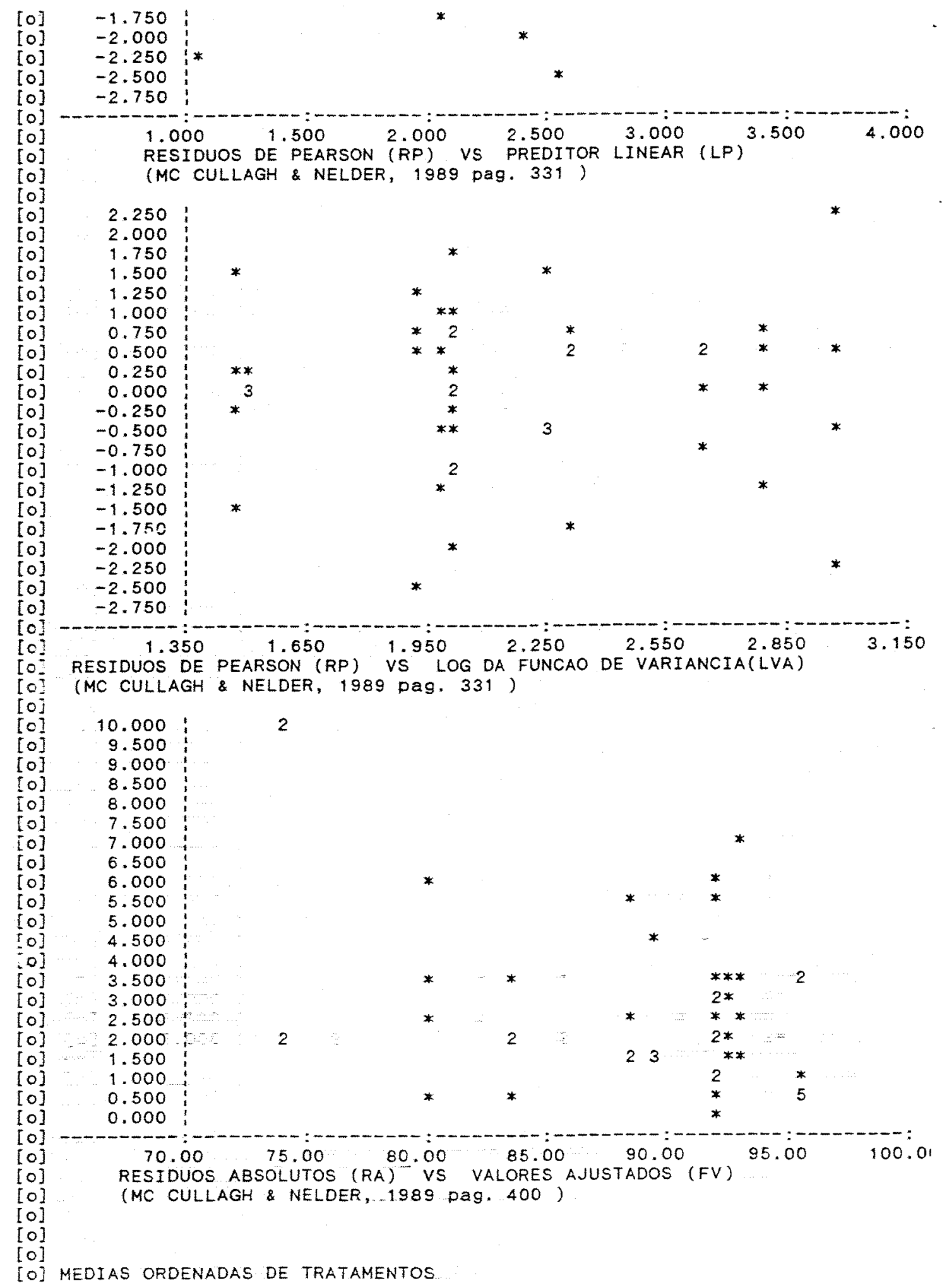




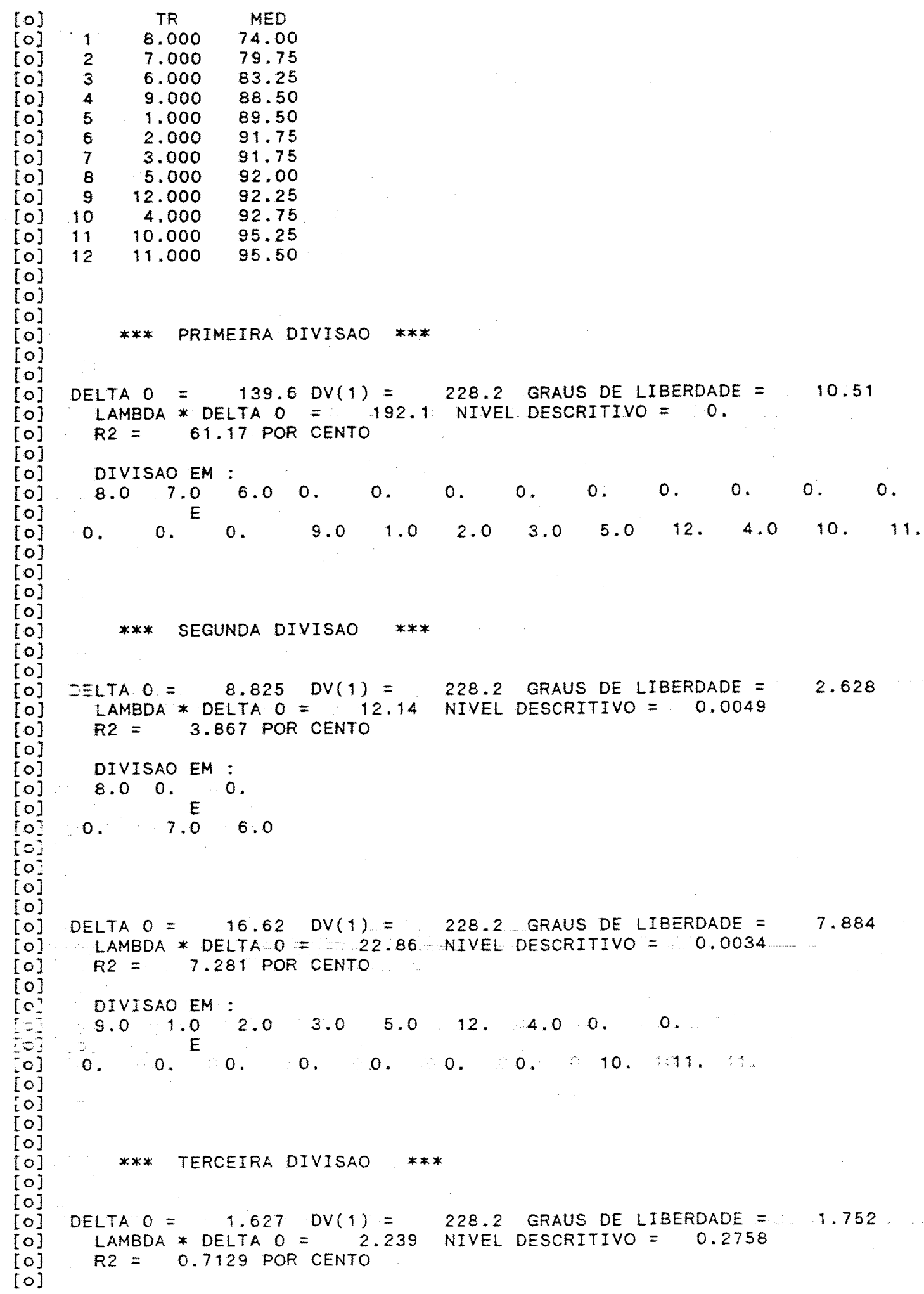


194.

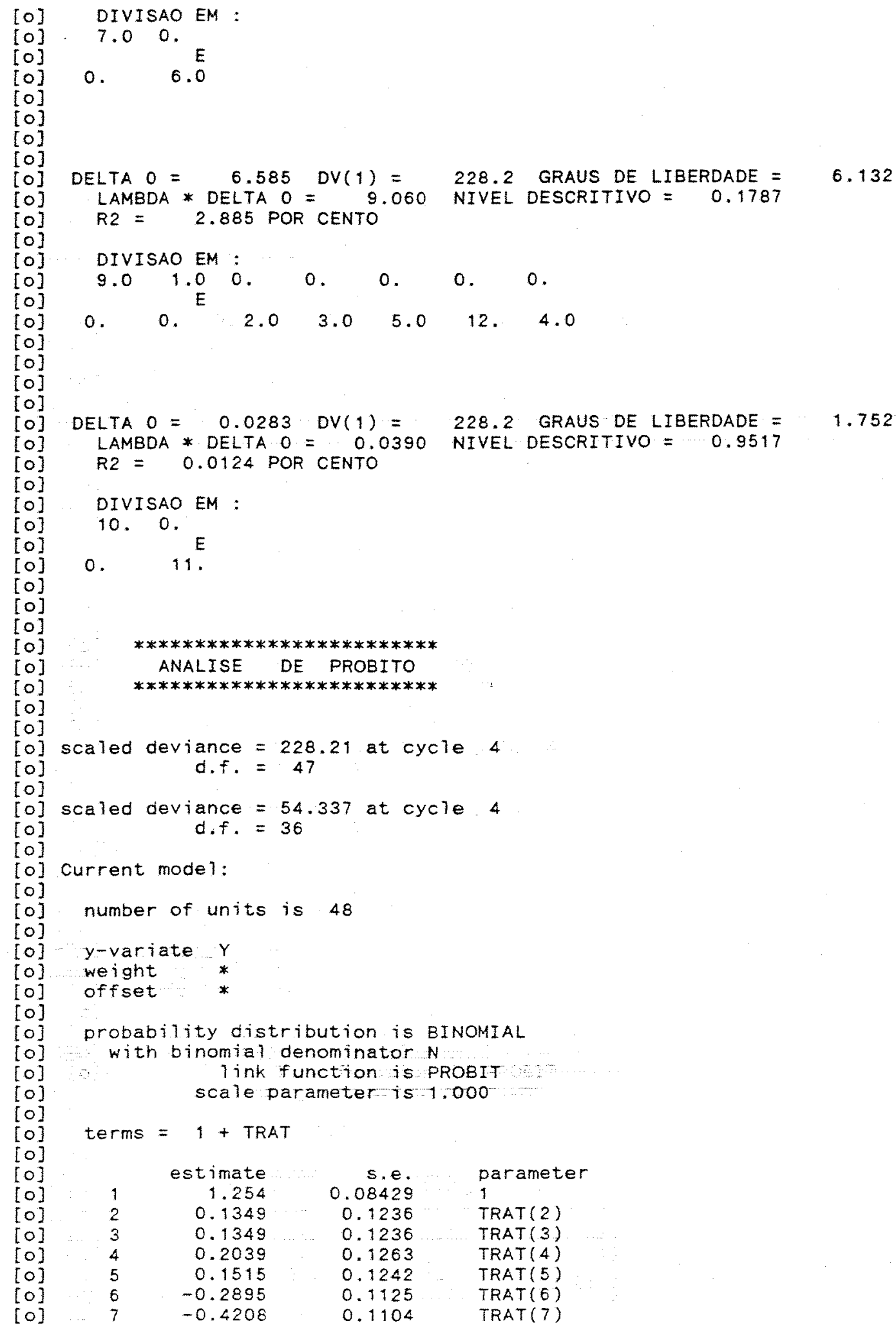




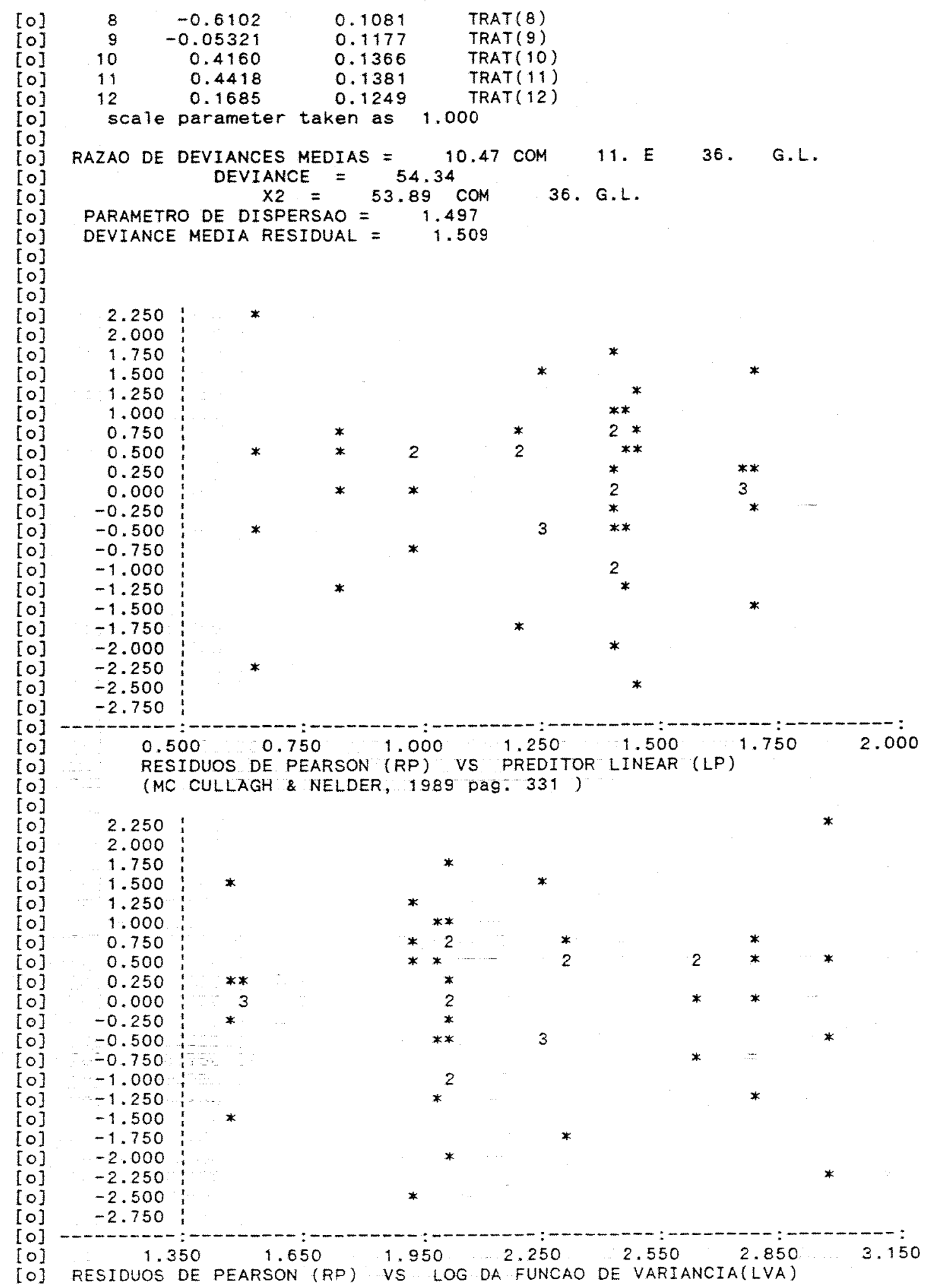


196.

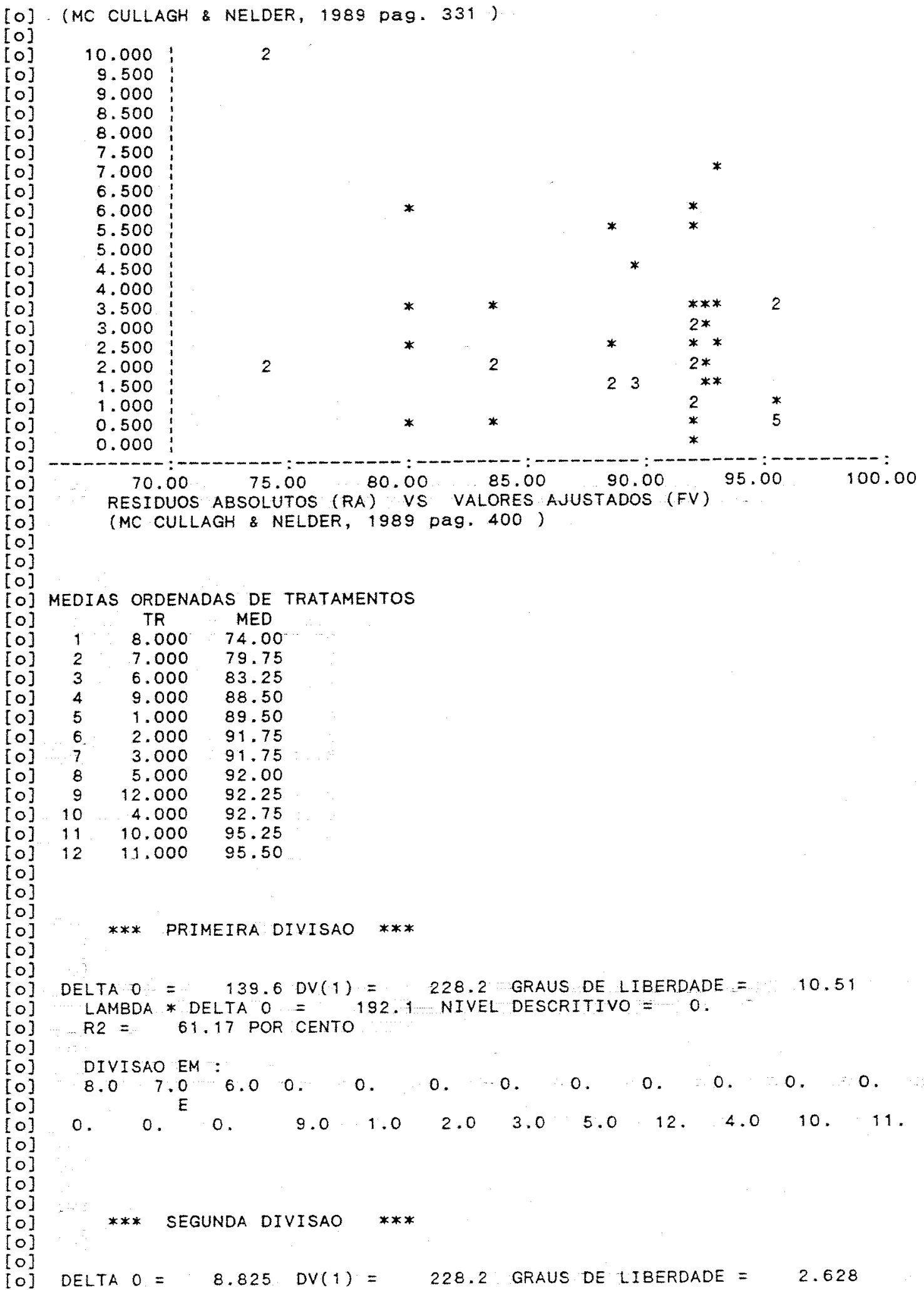




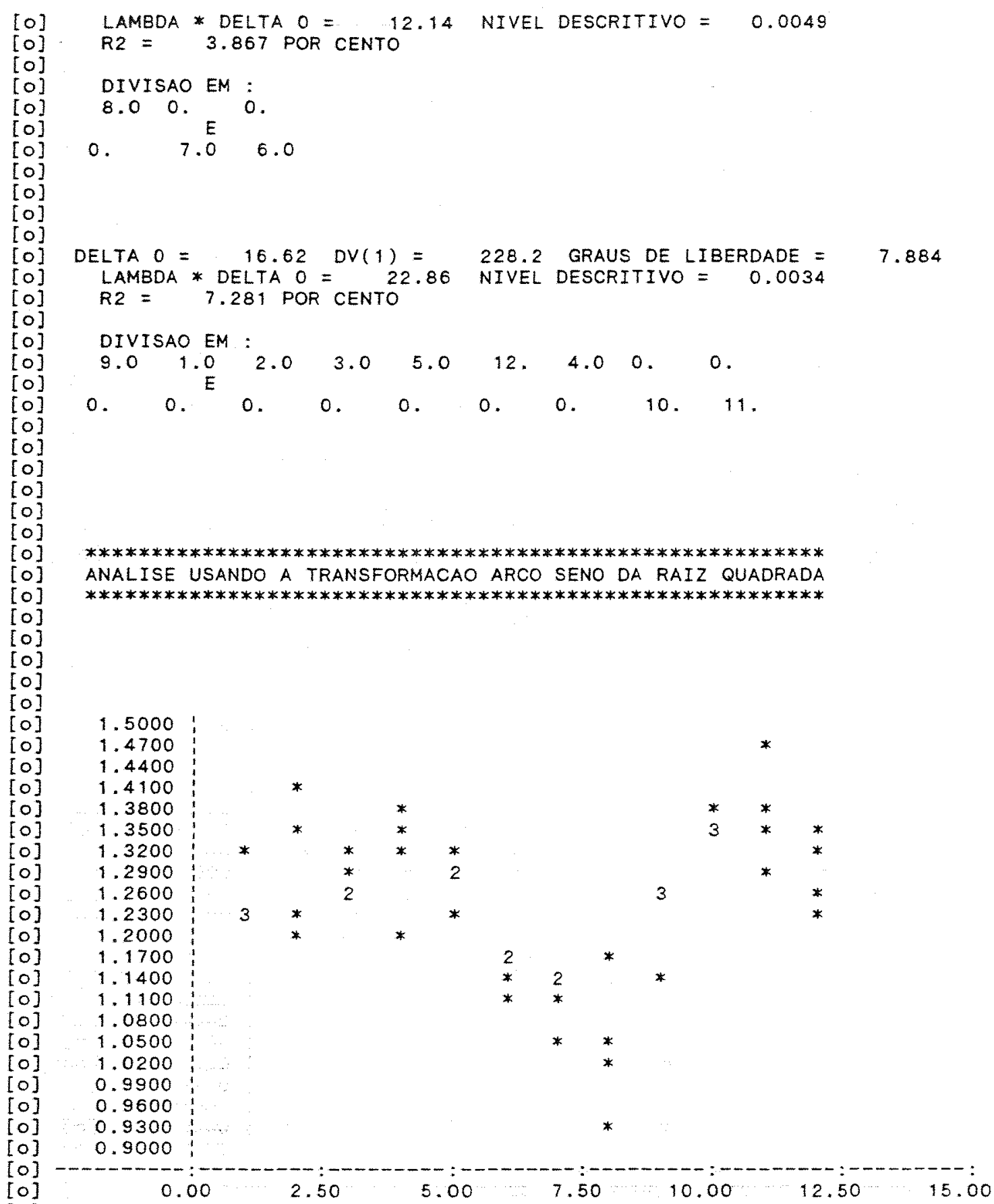

[0] GRAFICO DO VALOR OBSERVADO TRANSFORMADO CONTRA TRATAMENTO

$[0]$

[0]

[0]

[o]

[0]

[0]

[0]

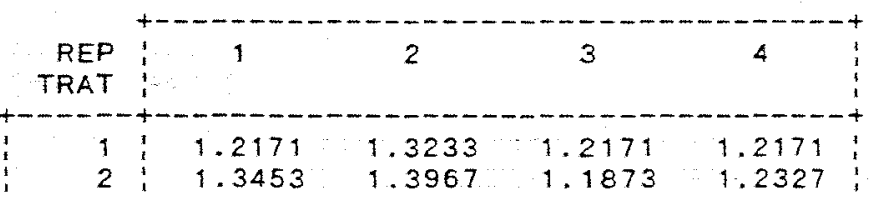




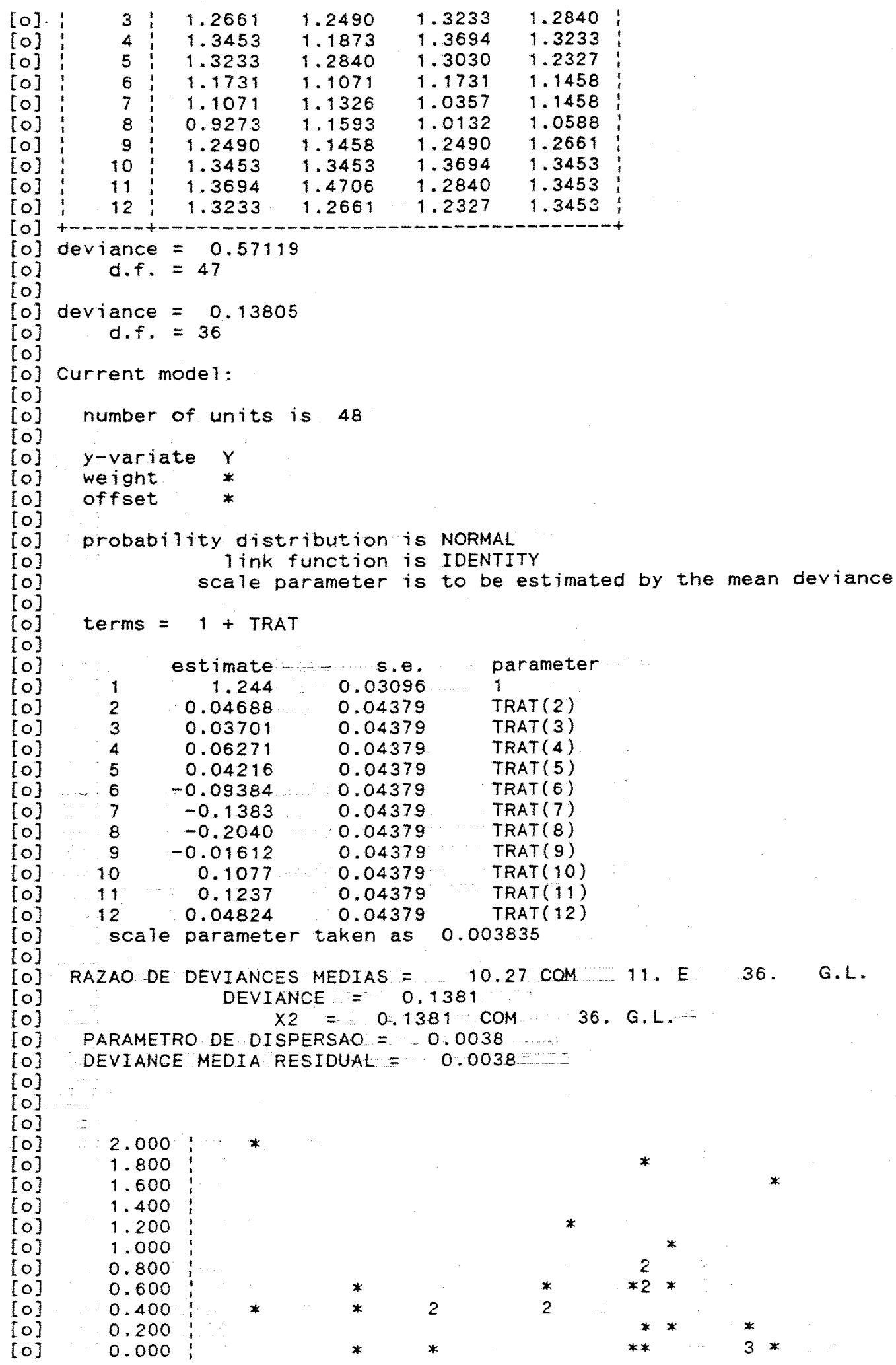


199.

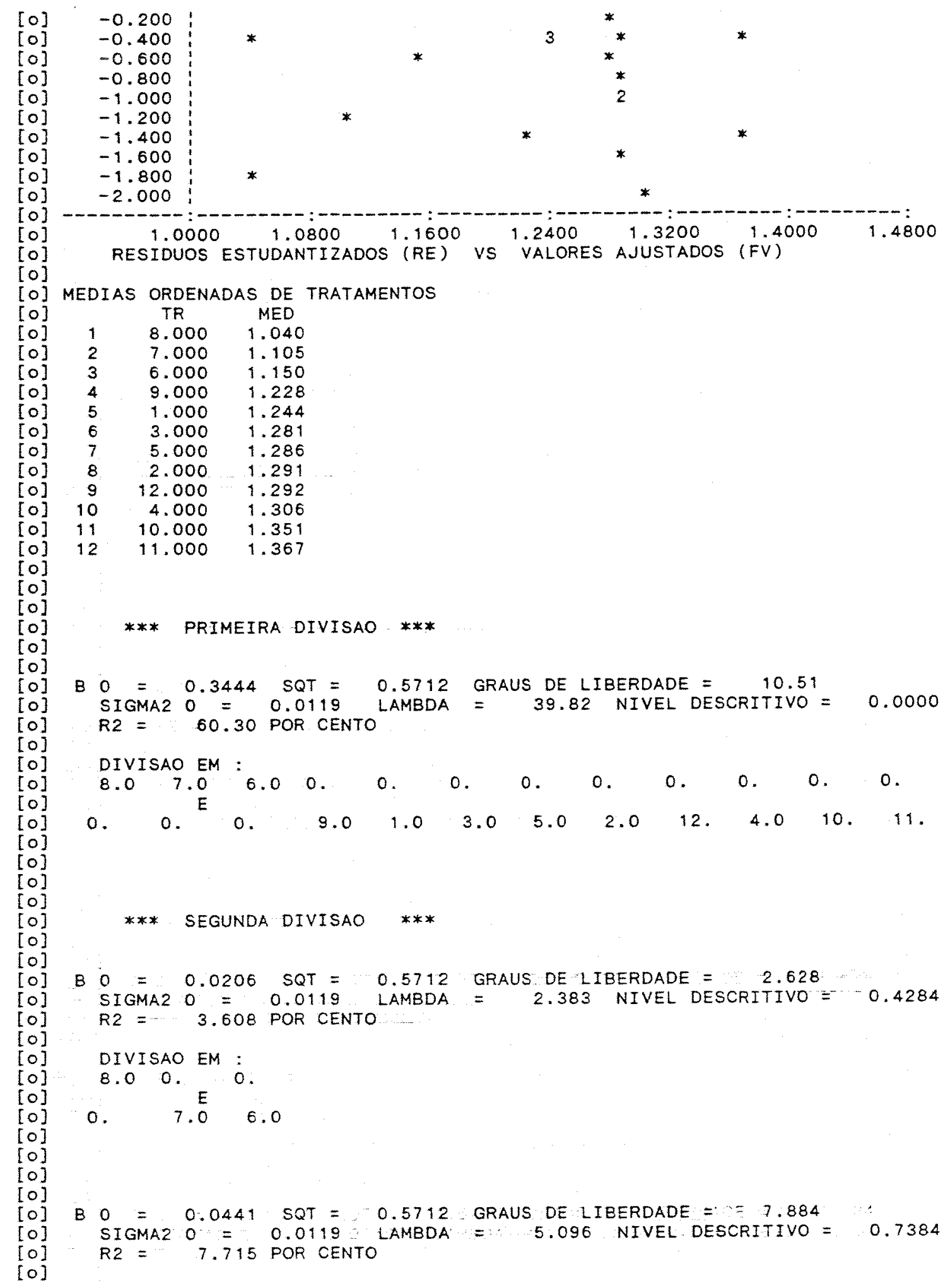


200.

[0] DIVISAO EM :

$[0] \quad \begin{array}{lllllllllll} & 9.0 & 1.0 & 3.0 & 5.0 & 2.0 & 12 . & 4.0 & 0 . & 0 .\end{array}$

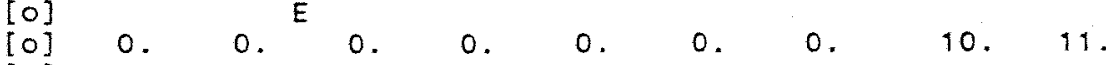

[0]

[0] 